Universidad

Politécnica

de Cartagena
Universidad

Politécnica

de Cartagena
Campus

de Excelencia

Internacional

TÉCNICAS ACÚSTICAS NO LINEALES PARA LAS COMUNICACIONES SUBMARINAS

Tecnologías de la Información y las Comunicaciones

María Mercedes Campo Valera

Directores: Ivan Felis Enguix Isidro Villó Pérez

Cartagena, 6 de julio de 2020 



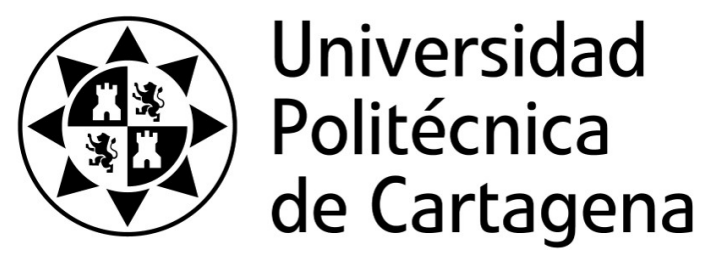

\title{
Técnicas acústicas no lineales para las comunicaciones submarinas
}

\author{
Julio de 2020
}

\section{María Mercedes Campo Valera \\ Tesis Doctoral}

Directores: Dr. Ivan Felis Enguix

Dr. Isidro Villó Pérez 



\section{CONFORMIDAD DE SOLICITUD DEAUTORIZACIÓN DE DEPÓSITO DE}

\section{TESIS DOCTORAL POR EL DIRECTOR DE LA TESIS}

Drs. Ivan Felis Enguix e Isidro Villó Pérez Directores de la Tesis doctoral: Técnicas acústicas no lineales para las comunicaciones submarinas.

\section{INFORMA:}

Que la referida Tesis Doctoral, ha sido realizada por $D^{a}$. María Mercedes Campo Valera dentro del Programa de Doctorado Tecnologías de la Información y Comunicaciones, dando nuestra conformidad para que sea presentada ante el Comité de Dirección de la Escuela Internacional de Doctorado para ser autorizado su depósito.

Informe positivo sobre el plan de investigación y documento de actividades del doctorando emitido por el Director (RAPI).

La rama de conocimiento en la que esta tesis ha sido desarrollada es:

$\square$ Ciencias

$\square \quad$ Ciencias Sociales y Jurídicas

$\square \quad \underline{\text { Ingeniería y Arquitectura }}$

En Cartagena, a 18 de junio de 2020

LOS DIRECTORES DE LA TESIS

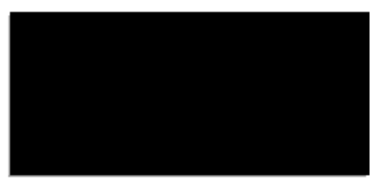

Ivan Felis Enguix

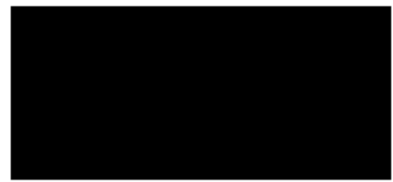

Isidro Villó Pérez 


\section{CONFORMIDAD DE DEPÓSITO DE TESIS DOCTORAL POR LA COMISIÓN ACADÉMICA DEL PROGRAMA}

Dr. Jorge Larrey Ruiz, Presidente de la Comisión Académica del Programa: Tecnologías de la Información y Comunicaciones.

\section{INFORMA:}

Que la Tesis Doctoral titulada, "Técnica acústicas no lineales para las comunicaciones submarinas", ha sido realizada, dentro del mencionado Programa de Doctorado, por $\mathrm{D}^{\mathrm{a}}$. María Mercedes Campo Valera, bajo la dirección y supervisión de los Drs. Ivan Felis Enguix e Isidro Villó Pérez.

En reunión de la Comisión Académica, visto que en la misma se acreditan los indicios de calidad correspondientes y la autorización de los Directores de esta, se acordó dar la conformidad, con la finalidad de que sea autorizado su depósito por el Comité de Dirección de la Escuela Internacional de Doctorado.

Evaluación positiva del plan de investigación y documento de actividades por el Presidente de la Comisión Académica del programa (RAPI).

La Rama de conocimiento por la que esta tesis ha sido desarrollada es:

$\begin{array}{ll}\square & \text { Ciencias } \\ \square & \text { Ciencias Sociales y Jurídicas } \\ \underline{\mathrm{X}} & \underline{\text { Ingeniería y Arquitectura }}\end{array}$

En Cartagena, a 18 de junio de 2020

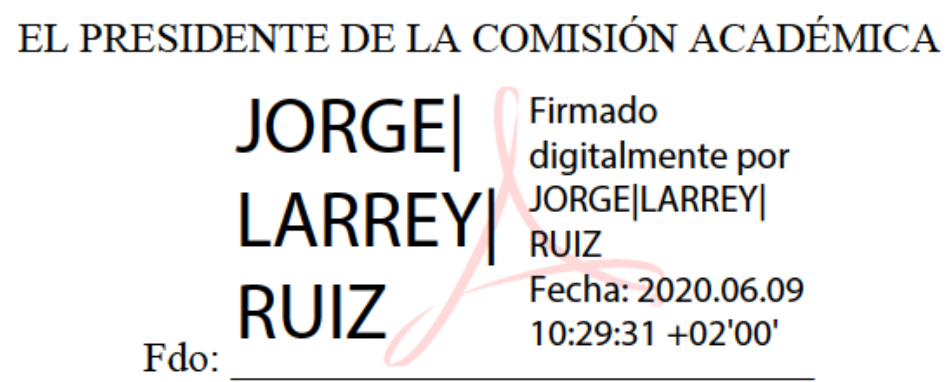


En memoria de la mujer con sonrisa soñadora...

A mi madre 



\section{Agradecimientos}

Quiero agradecer a mis directores. A Ivan, el cual tuve la oportunidad de tener como compañero en mis inicios de la tesis y trabajar en el laboratorio codo con codo, en el grupo de investigación encabezado por Miquel Ardid, hasta convertirse en mi director. A Isidro, trataré de ser breve contigo, pero... se que me costará. Conocerte en el proceso de mi tesis ha sido especial. Llegaste en el momento preciso, el trabajar juntos desde el respeto y la pasión que le pusiste a esta tesis permitiste que este invierno se transformara en colores. El café por la mañana, la comida, otro café-té y luego una charla mas o menos breve, hicieron dinámico este duro proceso. He aprendido mucho a tu lado. Gracias por ayudarme en los desarrollos matemáticos del primer capítulo. Eres una máquina, ¡Mil gracias!.

A Ramón Ruiz Merino, por darme la bienvenida en la UPCT y guiarme con cada consejo y palabra de aliento.

A Pablo Cervantes, quien fue el primer contacto que hice con el (CTN). Al Centro Tecnológico Naval y del Mar (CTN) y a su directora Noelia Ortega Ortega, por permitirme realizar los experimentos en los diferentes emplazamientos, tales como el laboratorio de acústica, la balsa troncocónica con su sistema de posicionamiento y los equipos para medir.

A Hamid Er-rachi, del (CTN), por estar atento en las mediciones experimentales de mi tesis, y dejar tu trabajo para mover el sistema de posicionamiento cuando tenía que medir en la balsa. Por todos esos momentos de compañía.

A mis chicos del E-201 de la UPV. Ivan Herrero y Ahmed Mehrem gracias por hacer divertidas las mañanas en el despacho. A Dídac Tortosa, fue bonito empezar mi etapa del doctorado contigo, extraño las noches de pizza y curro en el laboratorio, hacer 
ciencia contigo ha sido lo mejor. A mi querido Luis Salmerón, gracias por sacarme siempre de apuros, por enseñarme cosas de programación y por trasnochar conmigo desde la distancia ayudándome a resolver mis inquietudes, eres un sol, no cambies. A todos, son unos amigos increíbles.

A mis compañeros de despacho de la UPCT. Carlos Toledo, siempre atento y presto a escucharme. A Rodo López y José Abad, por las mañanas de relax en la cafetería que tanto necesité. A Chiraz Ajmi, por interrumpirme siempre que necesitaba un respiro, por soportarme y estar ahí aun cuando quería estar sola.

A mis queridos amigos que en el pasado fueron mis profesores de la carrera, Luis Hermida y Luis Jorge Herrera, siempre prestos a escucharme lo bueno y lo no tan guay en esta etapa.

A mis amigas. Cindy Consuegra, María Paola Torres, Laura Numa, Yaneth Bautista, Juanis Aldana, Marjorie Medina, Mari Escobar, Ayda Roldán, Liliana Cuesta y Lili Sanchez, que desde la distancia y de diferentes partes del mundo, nos unió la partida de mi madre justo cuando empezaba este camino. No las cambio.

A mi querida Yenys Cuesta, por las canas que te saqué en estos 4 años, por tanto tiempo robado, por hacerme crecer.

A todos aquellos que han llegado en mi camino en estos últimos meses, gracias por alentarme y hacerme ver lo bonito de la vida aun en medio de los claros y oscuros.

A mi familia, siempre a mi lado. Mi prima Luz Angela, a mis tias, Lyra y Conde, por el apoyo incondicional. A mi padre, mis hermanos y sobrina; Benjamín, Lili, Cristian y Valentina, porque muchas veces les robé el tiempo que teníamos para charlar desde la distancia por estar siempre con la tesis, por el apoyo emocional que me brindaron cuando la pasaba mal en este camino. Espero poder verles pronto. Los amo.

Con todos ustedes este proceso ha sido mas llevadero. Nuevamente, ¡Gracias!.

Y... este cuento no ha terminado. 


\section{Resumen}

Las comunicaciones en entornos submarinos se han convertido en un campo de investigación de gran interés en los últimos años por sus múltiples aplicaciones como, por ejemplo, monitorizar el medio marino para mantener la biodiversidad, perfilar el subfondo marino y avanzar en su conocimiento, controlar la navegación y el tránsito naval, defensa y seguridad, entre otros. Dado que las ondas acústicas presentan ciertas ventajas para su propagación en el medio marino, el desarrollo de transductores acústicos submarinos ha experimentado un aumento significativo como tecnologías de transmisión para sistemas de comunicación subacuática. Sin embargo, la propagación de las ondas en el mar tiene limitaciones debido a la dispersión geométrica y absorción, la presencia de ruido, y especialmente, el efecto multicamino. Todos estos mecanismos pueden llegar a provocar una distorsión en la señal recibida, en comparación con la señal emitida.

En aras de salvar este efecto no deseado, en esta tesis se plantean métodos de comunicación basados en el fenómeno de propagación no lineal llamado efecto paramétrico, que permite una comunicación directiva mediante el uso de transductores de alta frecuencia y alta intensidad, cuyas ondas no lineales dan lugar, en su propagación, a la aparición de bajas frecuencias en el medio. Con esto, se prevén varias ventajas: comunicarse solo en la dirección deseada, y disminuir en su mayoría las múltiples reflexiones que podrían empeorar la calidad de la comunicación en aguas poco profundas. Además, en la presente tesis se aborda una forma particular de modulación basada en la concatenación de señales de banda ancha tipo sweep (barridos en frecuencia), en contraste con las técnicas clásicas de modulación tipo seno (tonos). Este tipo de modulación, estudiada desde el punto de vista del efecto paramétrico, permitirá un elevado grado de detección y disminución de tasas de error mejorando, aún más, las prestaciones de la comunicación. 
A pesar de las diversas aplicaciones de este fenómeno paramétrico en las comunicaciones acústicas, su uso en otros ámbitos de la ingeniería no están lo suficientemente extendidas, debido a que la conversión de energía del paramétrico es ineficaz. Sin embargo, en los últimos años, a causa de la diversidad de nodos en las redes de comunicaciones acústicas submarinas y al aumento de la empleabilidad de equipos no tripulados $\left(\mathrm{UUV}^{1}\right)$ de gran tamaño, se empiezan a abordar los problemas de suministro de energía. Por lo tanto, las comunicaciones acústicas paramétricas generan mayor interés en su estudio y aplicación, utilizándose tanto en aplicaciones de detección/imagen así como para otros propósitos y desarrollos.

Esta tesis consiste en 5 capítulos. El primero, trata de los fundamentos teóricos de la acústica no lineal, prestando atención a aquellos fenómenos que conciernen a la generación paramétrica y sus características, aplicado al presente problema de las comunicaciones, sentando las bases para su entendimiento y, posteriormente, utilizarlo en el desarrollo de esta tesis. En segundo lugar, se estudian las técnicas de modulación empleadas en las comunicaciones acústicas submarinas y su implementación mediante el efecto paramétrico. El tercer capítulo, aborda las técnicas de detección y procesado de señales acústicas utilizadas para analizar las señales medidas y extraer sus características principales. En cuarto lugar, se caracteriza el campo acústico generado por el transductor utilizado en los experimentos, que aborda tanto su componente lineal como no lineal (efecto paramétrico). Por último, se comparan los resultados obtenidos de utilizar las diferentes modulaciones estudiadas, tanto las clásicas basadas en senos (tonos), como las propuestas mediante sweeps (barridos frecuenciales), todas ellas bajo el fenómeno de propagación no lineal, para así obtener su capacidad de detección y calidad de cada una de las comunicaciones acústicas estudiadas.

\footnotetext{
${ }^{1}$ Unmanned Underwater Vehicles
} 


\section{Abstract}

Communications in underwater environments have become a field of research of great interest in recent years due to its applications, these include, for example, monitoring the marine environment to maintain biodiversity, mapping the profile of the seabed and increasing the knowledge about them, controlling the ship traffic, defence and security, among others. Since acoustic waves have certain advantages for their specific propagation characteristics in the marine environment, the development of underwater acoustic transducers has experienced a significant increase, as it happens with as transmission technologies for underwater communication systems. However, underwater acoustic has several limitations due to the geometric spreading (divergence effect) and absorption, the presence of noise, and especially, the multipath effect. All the aforementioned mechanisms might cause a significant distortion in the received signal, when compared to the emitted signal.

In order to overcome this unwanted effect, in this $\mathrm{Ph} . \mathrm{D}$. investigates distinct communication methods, under the so-called parametric effect conditions (non-linear propagation phenomena). These non-linear waves give rise to the occurrence of low frequencies in the middle of their propagation. Eventually, the parametric effect allows a directive communication by using high-frequency and high intensity transducers. With this, several advantages are foreseen: directive communications, and most important, reduction the multiple reflections that could worsen the quality of communication in shallow waters. Furthermore, this work deals with a particular form of modulation based on the concatenation of broadband sweep signals, in contrast to the classic techniques of sine-type modulation. This type of modulation, studied from the point of view of the parametric effect, will allow a high degree of detection and a reduction in communication error rates, further improving the overall underwater acoustic communication performance. 
Despite its multiple applications of this parametric phenomenon in acoustic communications, its use in other areas of engineering are not widespread enough, mainly due to the low energy conversion because associated to the parametric operation. However, problems of energy supply are being highlighted in recent years, due to the diversity of nodes in the underwater acoustic communication networks and the increase in the employability of large unmanned equipment $\left(\mathrm{UUV}^{2}\right)$. Therefore, the study of parametric acoustic communications are gaining more and more interest, with application to both detection/acoustic imaging, as well as other interesting purposes.

This work consists of 5 parts. The first one, deals with the theoretical foundations of nonlinear acoustic, paying attention to those phenomena that concern the parametric generation and its characteristics. Special emphasis is given to the present problem of communications, laying the foundations for its understanding and, subsequently, using it in the development of this thesis. Second, the modulation techniques used in underwater acoustic communications and their implementation through the parametric effect are studied. The third part deals with the acoustic signal detection and processing techniques used to analyze the measured signals and extracting their main characteristics. Fourth, the acoustic field generated by the underwater transducer used in the experiments is characterized, tackling with both its linear and non-linear components (parametric effect). Finally, the results obtained from using the different studied modulations, both classic single-tone and the frequency sine-sweep schemes, are compared, all under the phenomenon of non-linear propagation and, thus, obtain their ability to detection and quality of each of the communications studied.

\footnotetext{
${ }^{2}$ Unmanned Underwater Vehicles
} 


\section{Tabla de contenido}

Agradecimientos

Resumen

Abstract

1 Principios de la acústica no lineal 1

1.1 Efectos asociados a la propagación no lineal . . . . . . . . . . 2

1.1.1 Propagación de un pulso gaussiano con condiciones iniciales. . . . . . . . . 5

1.1.2 Propagación de una onda armónica con condiciones iniciales . . . . . . . . . 9

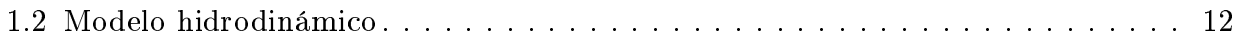

1.2.1 Aproximación de $1^{\circ}$ orden (Acústica lineal) . . . . . . . . . . . . 15

1.2 .2 Aproximación de $2^{\circ}$ orden (Acústica no lineal) . . . . . . . . . . . . 16

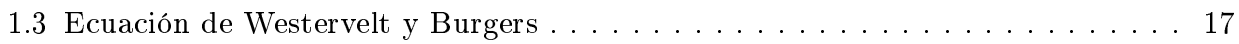

1.4 Problemas de contorno . . . . . . . . . . . . . . . . . . . 21

1.4.1 Excitación armónica mono-frecuencia en un medio no viscoso . . . . . . . . . . . 22

1.4.2 Excitación armónica bi-frecuencia en un medio no viscoso . . . . . . . . . . . 26

1.4.3 Excitación armónica mono-frecuencia en un medio viscoso. . . . . . . . . . . . 29 
1.4.4 Excitación armónica bi-frecuencia en un medio viscoso . . . . . . . . . . . . . 31

1.4.5 Excitación modulada en un medio viscoso . . . . . . . . . . . . . . . . 32

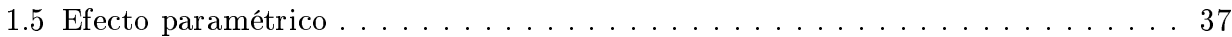

1.6 Ecuación KZK y solución de Berktay . . . . . . . . . . . . . . . . . . . 40

1.6.1 Comparación teórica y experimental con el modelo de Moffett y Mellen . . . . . 46

Desarrollo teórico y experimental . . . . . . . . . . . . . . 47

1.7 Introducción a la acústica submarina . . . . . . . . . . . . . . . . 49

1.7 .1 Fenomenología del canal acústico submarino . . . . . . . . . . . . . . . . . 49

Velocidad de propagación en el mar . . . . . . . . . . . . . . 50

Perfil de velocidad . . . . . . . . . . . . . . . . . . 51

Absorción .......................... 52

1.7.2 Propagación en aguas poco profundas . . . . . . . . . . . . . . 55

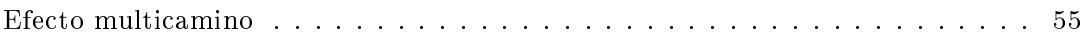

Modelado de la propagación . . . . . . . . . . . . . . . . . 55

1.7.3 Consideraciones sobre el ruido de fondo . . . . . . . . . . . . . . . 57

2 Comunicaciones acústicas submarinas 61

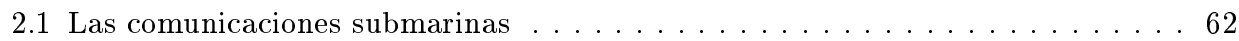

2.1 .1 Comunicaciones por radiofrecuencia . . . . . . . . . . . . . 62

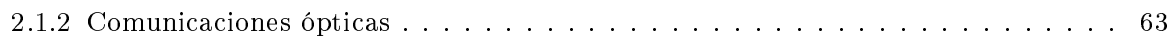

2.1.3 Comunicaciones acústicas . . . . . . . . . . . . . . . . . . . . 64

2.1 .4 Comparativa. . . . . . . . . . . . . . . . . . . . 6 64

2.2 Las comunicaciones acústicas submarinas . . . . . . . . . . . 65

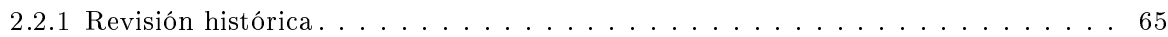

2.2 .2 Aplicaciones . . . . . . . . . . . . . . . . . . 67

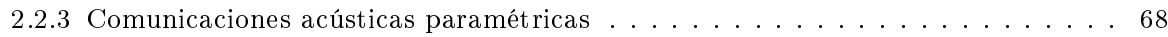

2.3 Técnicas de modulaciones . . . . . . . . . . . . . . . . . 71

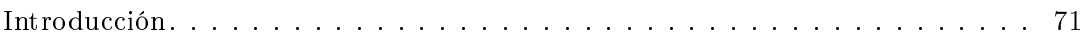

2.3.1 Modulaciones analógicas _. . . . . . . . . . . . . . . . . 72

Modulación en amplitud $(\mathrm{AM}) \ldots \ldots \ldots \ldots \ldots \ldots$

2.3.2 Modulaciones digitales. . . . . . . . . . . . . . . . 73

Modulación por desplazamiento de amplitud (ASK) . . . . . . . . . . 75

Modulación por desplazamiento de frecuencia (FSK) . . . . . . . . . . 76

Modulación por desplazamiento de fase $(\mathrm{PSK}) \ldots \ldots \ldots \ldots$

Modulaciones Multinivel M-Aria . . . . . . . . . . . . . 77 
2.4 Modulaciones para señales no lineales tipo paramétrico. . . . . . . . . . 79

2.4.1 Introducción a las modulaciones para comunicaciones no lineales. . . . . . . . . 79

Proceso directo e inverso . . . . . . . . . . . . . . . . . . . . 79

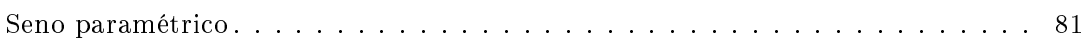

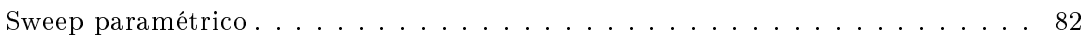

3 Procesamiento de señales acústicas 85

3.1 Técnicas para el procesado de señales . . . . . . . . . . . . 86

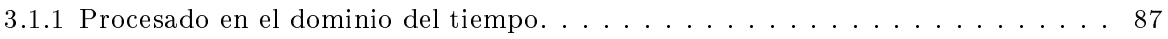

El dominio del tiempo (transformada identidad) . . . . . . . . . . . 87

Parametrización en tiempo . . . . . . . . . . . . . . . . . . 88

3.1 .2 Procesado en el dominio de la frecuencia . . . . . . . . . . . . . . . . . 89

El dominio de la frecuencia (Transformada de Fourier) . . . . . . . . . . . 89

Parametrización en frecuencia . . . . . . . . . . . . . . . . . . 89

3.1 .3 Procesado en el dominio de la correlación . . . . . . . . . . . . . . . . 90

La correlación cruzada . . . . . . . . . . . . . . . . . . . . 90 90

Parametrización en la correlación . . . . . . . . . . . . . . . . . . 91

3.2 Aplicación de las técnicas a señales lineales o de calibración. . . . . . . . . . . 92

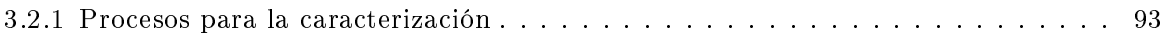

3.2 .2 Resultados de caracterizar un transductor . . . . . . . . . . . . . . . . . 93

Análisis de señales . . . . . . . . . . . . . . . . . . . . . . . . 93

3.3 Aplicación de las técnicas a señales no lineales de comunicación . . . . . . . . 97

3.3.1 Procesos para la caracterización . . . . . . . . . . . . . . . . . . . 97

3.3.2 Resultados de señales paramétricas tipo seno . . . . . . . . . . . . . 99

Análisis de señales para el haz primario . . . . . . . . . . . . . . . . 99

Análisis de señales para el haz secundario . . . . . . . . . . . . . . . . 101

3.3.3 Resultados de señales paramétricas tipo sweep . . . . . . . . . . . . . . . . 103

Análisis para el haz primario. . . . . . . . . . . . . . . . . . . 103

Análisis para el haz secundario . . . . . . . . . . . . . . . 105

4 Caracterización del campo acústico de un transductor $\quad 107$

4.1 Parámetros de caracterización en régimen lineal. . . . . . . . . . . . . . . 108

Impedancia eléctrica . . . . . . . . . . . . . . . . . . . . 108

Sensibilidad acústica. . . . . . . . . . . . . . . . . . . . . . . . . . 109

Directividad . . . . . . . . . . . . . . . . . . . . . . 110 
4.2 Parámetros de caracterización en régimen no lineal . . . . . . . . . . . . . 111

Directividad . . . . . . . . . . . . . . . . . . . . . . . . . . 111

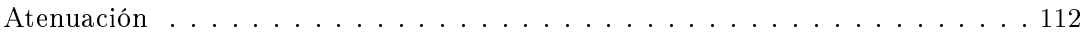

Variación de voltaje . . . . . . . . . . . . . . . . . . . . . 113

4.3 Montaje experimental . . . . . . . . . . . . . . . . . . . 113

4.3 .1 Régimen lineal. . . . . . . . . . . . . . . . . . . . . . . . . . . . . . 114

Montaje de impedancia eléctrica . . . . . . . . . . . . . . . . . . . . . 114

Montaje de TVR en piscina . . . . . . . . . . . . . . . . . . 114

4.3 .2 Régimen no lineal. . . . . . . . . . . . . . . . . . . . . . . . . . 116

Montaje en piscina . . . . . . . . . . . . . . . . . . . 116

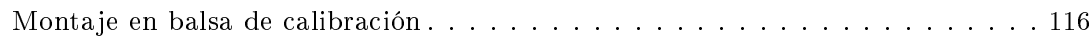

4.3 .3 Configuración de las medidas. . . . . . . . . . . . . . . . . . . 116

4.4 Resultados de caracterizar un transductor en régimen lineal. . . . . . . . . . . 118

4.4 .1 Admitancia . . . . . . . . . . . . . . . . . . . . . . . . . . . . 118

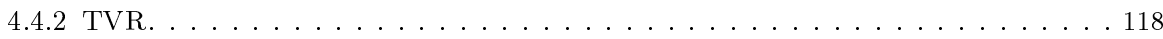

4.5 Resultados de caracterizar un transductor en régimen no lineal (paramétrico). . 119

4.5.1 Medidas con senos paramétricos . . . . . . . . . . . . . . . . . . . . . . 120

Directividad . . . . . . . . . . . . . . . . . . . . . . . . 120

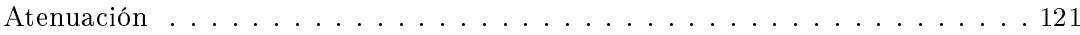

Variación de voltaje . . . . . . . . . . . . . . . . . . . . 121

4.5.2 Medidas con sweeps paramétricos . . . . . . . . . . . . . . . . . . . . . 122

Directividad . . . . . . . . . . . . . . . . . . . . . . 123

Atenuación . . . . . . . . . . . . . . . . . . . . . . . 124

Variación de voltaje . . . . . . . . . . . . . . . . . . . . . . 124

5 Aplicación a las comunicaciones acústicas $\quad 127$

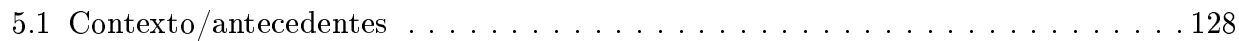

5.2 Capacidad de detección . . . . . . . . . . . . . . . . . . . . . 129

5.2 .1 Modulaciones con senos y sweeps paramétricos . . . . . . . . . . . . . . 130

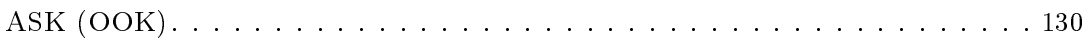

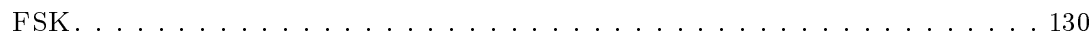

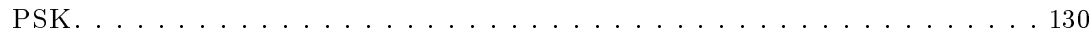

Sweeps . . . . . . . . . . . . . . . . . . . . . . . . 131

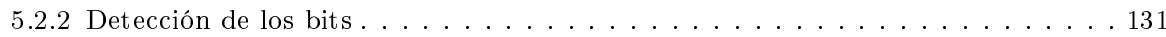


5.2 .3 Bit Error $(\mathrm{BER}) \ldots \ldots \ldots \ldots \ldots \ldots \ldots \ldots$

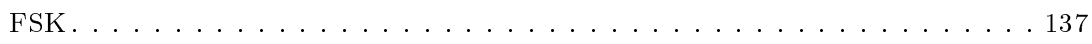

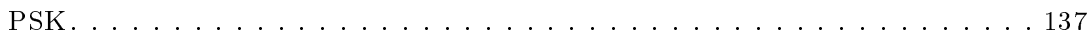

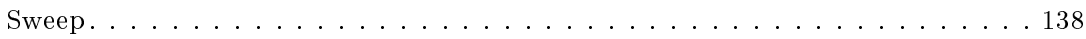

$\begin{array}{ll}\text { Conclusiones } & 141\end{array}$

$\begin{array}{ll}\text { Contribuciones científicas } & 145\end{array}$

$\begin{array}{ll}\text { A Notas matemáticas } & 147\end{array}$

A.1 Ecuación de estado . . . . . . . . . . . . . . . . . . . . 147

A.2 Formación del choque en problemas de frontera . . . . . . . . . . . 148

A.3 Solución Bessel-Fubini caso mono-frecuencia. . . . . . . . . . . . . . . . 149

B Efectos asociados a la viscosidad 153

B.1 Ecuación de Westervelt y Burgers viscosa . . . . . . . . . . . . . 153

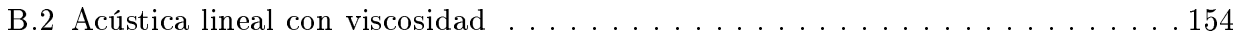

$\begin{array}{ll}\text { Bibliografía } & 155\end{array}$ 



\section{Capítulo 1 \\ Principios de la acústica no lineal}

La acústica es la rama de la física que estudia la generación, propagación y recepción del sonido. Esta ciencia cubre muchos campos y está íntimamente relacionada con varias áreas de la ingeniería. Desde el punto de vista matemático, el sonido se describe mediante ondas longitudinales de presión que se propagan a través de un fluido (gas o líquido). Estas ondas de presión se pueden utilizar como señales para transmitir información entre distintas regiones del espacio.

Dependiendo de la intensidad de la señal, los fenómenos acústicos se pueden clasificar en lineales y no lineales. En primer lugar, en el apartado 1.1, se estudia la formulación matemática del problema y así obtener las ecuaciones que gobiernan dicha propagación para, consecuentemente en el apartado 1.2, introducir el modelo hidrodinámico que da lugar, dependiendo del conjunto de ecuaciones que se aborden, al estudio de la acústica lineal y la no lineal. Posteriormente, en el apartado 1.3, se describe la ecuación de Westervelt y la Burguers que da inicio en los siguientes estudios, al desarrollo de los diferentes problemas de contorno explicados en el apartado 1.4. Con todo ello, se finaliza en el estudio de la generación paramétrica donde se describen aquellas características que serán útiles en el ámbito de las comunicaciones acústicas no lineales, tema central de esta tesis y así abordar el desarrollo de un modelo teórico contrastado con las medidas experimentales, que estudia la relación de las amplitudes del haz primario y el haz secundario.

En segundo lugar, y dado que esta tesis basa su interés en la acústica submarina, se tratan en el apartado 1.7, aquellos fenómenos concernientes al canal acústico submarino como son: la velocidad del sonido, absorción, además de, la propagación en aguas poco profundas. 


\subsection{Efectos asociados a la propagación no lineal}

La propagación de señales acústicas de pequeña amplitud está gobernada por la ecuación de onda lineal. Por simplicidad, se tratará el caso unidimensional. Para ondas progresivas de presión que se propagan hacia la derecha, esta ecuación se escribe como:

$$
\frac{\partial p(x, t)}{\partial t}+c_{o} \frac{\partial p(x, t)}{\partial x}=0
$$

donde $c_{o}$ es la velocidad del sonido medida en pequeña amplitud. En este grado de aproximación, dicha cantidad es una constante (para una temperatura dada). Por ejemplo, la velocidad de las ondas sonoras en el agua es $c_{o}=1481.44 \mathrm{~m} / \mathrm{s}$ a $20^{\circ} \mathrm{C}$. Como bien se sabe, en ausencia de condiciones de contorno (medio infinito), la solución general de la ecuación de onda de primer orden (1.1) es:

$$
p(x, t)=f\left(x-c_{o} t\right)
$$

donde $f(x)$ es una función dada por las condiciones iniciales del problema de Cauchy, esto es, $f(x)=p(x, 0)$.

Por ser $c_{o}$ una constante, la solución (1.2) tiene la propiedad de mantener su forma a lo largo de la propagación. En otras palabras, para cada tiempo $t$ la curva $f(x)$ se desplaza hacia la derecha sin deformación, una cantidad $c_{o} t$. Este comportamiento es característico en un régimen lineal.

Por otra parte, si la amplitud de la señal no es despreciable, el modelo lineal ya no es apropiado. En este caso, la propagación está gobernada por una ecuación de onda no lineal. Para describir los efectos debidos a la no linealidad, se tomará como punto de partida la ecuación de Burgers que es un modelo sencillo, pero a la vez importante de ecuación no lineal de primer orden uni-dimensional. En el próximo apartado, se obtendrá dicha ecuación a partir del modelo hidrodinámico. Por ahora, se limitará simplemente a tratar con sus soluciones.

El problema de Cauchy para la ecuación de Burgers se expresa como:

$$
\left\{\begin{array}{l}
\frac{\partial p(x, t)}{\partial t}+c(p) \frac{\partial p(x, t)}{\partial x}=0 \\
p(x, 0)=f(x)
\end{array}\right.
$$

donde $c(p)$ es una función dada que depende a su vez de la propia solución $p=p(x, t)$. 
Se observa, que la estructura matemática de la ecuación de Burgers (1.3a) es idéntica a la ecuación lineal (1.1) excepto que ahora la velocidad $c(p)$ ya no es una constante. Cuando $c$ depende de $p$ se tiene una ecuación de onda de primer orden no lineal.

Una primera consecuencia de la no linealidad es que el principio de superposición no se cumple, una segunda consecuencia es que los métodos utilizados para resolver ecuaciones diferenciales lineales(separación de variables, transformada de Laplace, de Fourier, función de Green, entre otros) no se pueden aplicar a problemas no lineales.

El problema de Cauchy (1.3a-1.3b) admite una solución que es conocida como solución $\mathrm{u}$ onda de Riemann y viene dada por:

$$
p(x, t)=f[x-c(p) t]
$$

La solución lineal (1.2) y la no lineal (1.4) tienen exactamente la misma forma pero comportamientos muy distintos. En efecto, la dependencia funcional de $c$ con $p$ implica que cada uno de los puntos de la curva inicial $p(x, 0)=f(x)$ se propagarán con diferentes velocidades. Este comportamiento queda ilustrado en la Figura 1.1. Si en el instante $t=0$ la onda tiene el perfil $p(x, 0)=f(x)$, pasado un tiempo $t$ la onda tendrá un perfil $p(x, t)$ distinto al inicial. Se habla entonces de una velocidad de onda local $c(p)$.

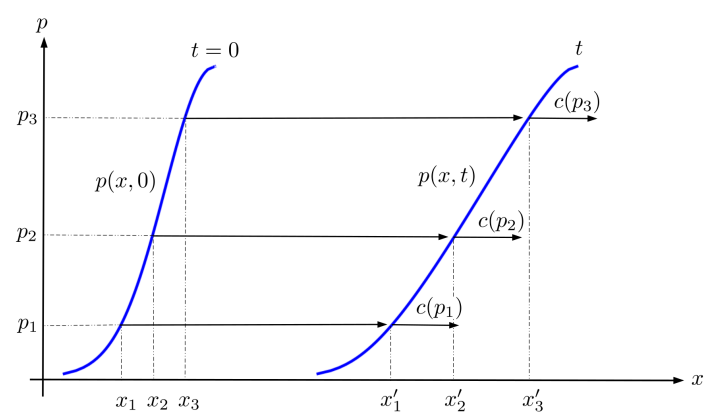

Figura 1.1: Cada $p_{i}$ se mantiene constante moviéndose con una determinada velocidad $c\left(p_{i}\right)$. En el instante $t$ la presión $p_{i}$ habrá recorrido una distancia $c\left(p_{i}\right) t$ siendo $x_{i}^{\prime}=x_{i}+c\left(p_{i}\right) t$

Como consecuencia de lo anterior, el perfil de onda se irá deformando hasta que la solución llega a ser multivaluada y, por lo tanto, carente de sentido físico (la solución debe ser una función univaluada de la posición). Se dice que, la onda se rompe (the wave breaking). Entonces, se considera una solución discontinua que se denomina onda de choque. La onda de choque, ocurre en una determinada posición $x_{s h}$ y en un 
determinado instante de tiempo $t_{s h}$ (the breaking time). La ecuación (1.3a) solamente será válida si $t \leq t_{s h}$. Para $t>t_{s h}$ la ecuación (1.3a) no describe apropiadamente el fenómeno físico y es necesario revisar algunas de las aproximaciones y suposiciones de partida. Matemáticamente, la existencia de esta singularidad puede comprobarse haciendo en (1.4) el cambio de variable:

$$
\xi=x-c(p) t \quad \Longrightarrow \quad p(\xi)=f(\xi)
$$

Usando la regla de la cadena se obtiene:

$$
\begin{aligned}
& \frac{\partial p}{\partial t}=\frac{d f}{d \xi} \frac{\partial \xi}{\partial t}=-\left(c+t \frac{d c}{d p} \frac{\partial p}{\partial t}\right) \frac{d f}{d \xi} \\
& \frac{\partial p}{\partial x}=\frac{d f}{d \xi} \frac{\partial \xi}{\partial x}=\left(1-t \frac{d c}{d p} \frac{\partial p}{\partial x}\right) \frac{d f}{d \xi}
\end{aligned}
$$

Despejando las derivadas primeras $\partial p / \partial t$ y $\partial p / \partial x$ resulta:

$$
\begin{aligned}
\frac{\partial p(x, t)}{\partial t} & =-\frac{c(p) f^{\prime}(\xi)}{1+c^{\prime}(p) f^{\prime}(\xi) t} \\
\frac{\partial p(x, t)}{\partial x} & =\frac{f^{\prime}(\xi)}{1+c^{\prime}(p) f^{\prime}(\xi) t}
\end{aligned}
$$

donde el índice prima (') denota derivadas respecto a sus variables. Estas derivadas divergen dando lugar a un tangente vertical $(t v)$, si los denominadores se hacen cero en algún lugar o tiempo. Esto ocurre si $1+c^{\prime}(p) f^{\prime}(\xi) t=0$. De donde:

$$
t_{t v}=\frac{-1}{c^{\prime}(p) f^{\prime}(\xi)}
$$

El valor más pequeño de $t_{t v}$ es lo que se considera $t_{s h}$,

$$
t_{s h}=\frac{-1}{c^{\prime}(p) f_{\min }^{\prime}(\xi)}
$$

o, equivalentemente,

$$
t_{s h}=\frac{1}{c^{\prime}(p)\left[-f^{\prime}(\xi)\right]_{\text {máx }}}
$$


En la práctica, el extremo de esta función (máx o mín) se puede obtener haciendo la segunda derivada igual al cero.

La distorsión de la onda debido a la no linealidad está inherentemente unida a un cambio en el espectro de frecuencias del perfil de onda inicial. El cambio del espectro con la propagación es otro de los efectos más notables de la no linealidad.

Con el objetivo de ilustrar estos conceptos y concretar más en ellos, se considera la solución explícita de dos problemas de valor inicial: la propagación de un pulso gaussiano y la propagación de una onda armónica. Más adelante se tratará el problema de contorno asociado al último de los casos anteriores.

\subsubsection{Propagación de un pulso gaussiano con condiciones iniciales}

Considerando el problema de Cauchy para la ecuación de Burgers cuyo perfil de onda inicial es un pulso gaussiano de amplitud $\mathcal{P}_{o}$, se tiene:

$$
\left\{\begin{array}{l}
\frac{\partial p(x, t)}{\partial t}+\left(c_{o}+a p\right) \frac{\partial p(x, t)}{\partial x}=0 \\
p(x, 0)=\mathcal{P}_{o} e^{-x^{2}}
\end{array}\right.
$$

donde $c_{o}>0$ y $a \geq 0$ son constantes que se suponen conocidas.

Según (1.3a) y (1.3b) resulta ser:

$$
\begin{aligned}
& c(p)=c_{o}+a p, \quad 0 \leq p \leq \mathcal{P}_{o} \\
& f(x)=\mathcal{P}_{o} e^{-x^{2}}
\end{aligned}
$$

en este caso se observa, que la velocidad de onda local $c(p)$ es una función lineal de $p$, y crece desde un valor mínimo $c_{o}$ (para $p=0$ ) hasta un valor máximo $c_{o}+a \mathcal{P}_{o}$ (para $p=\mathcal{P}_{o}$ ). A continuación, se considerarán los casos: $a=0$ y $a>0$.

- Caso lineal, $a=0$

Si $a=0$, el problema se reduce a un problema lineal cuya solución viene dada por $(1.2)$ :

$$
p(x, t)=f\left(x-c_{o} t\right)=\mathcal{P}_{o} e^{-\left(x-c_{o} t\right)^{2}}
$$


Por ser $c_{o}$ una constante, el perfil de onda gaussiano se propaga sin deformación con velocidad $c_{o}$ tal y como muestra la Figura 1.2.

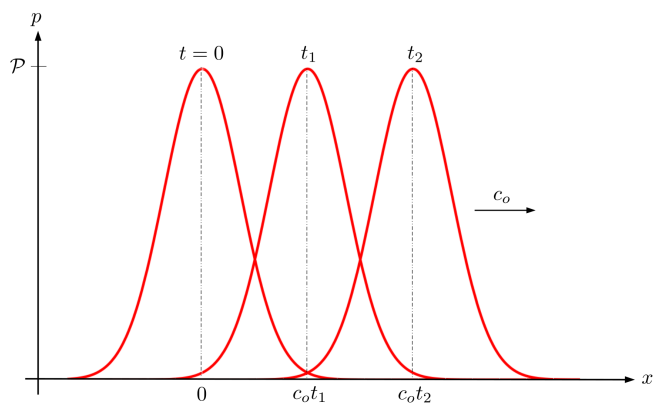

Figura 1.2: Propagación en régimen lineal. El pulso se propaga sin deformación

- Caso no lineal, $a>0$

Si $a>0$, la solución al problema de valor inicial (1.13) es una onda de Riemann dada por (1.4):

$$
p(x, t)=f[x-c(p) t]=f\left[x-\left(c_{o}+a p\right) t\right]
$$

Esto es:

$$
p(x, t)=\mathcal{P}_{o} e^{-[x-c(p) t]^{2}}=\mathcal{P}_{o} e^{-\left[x-\left(c_{o}+a p\right) t\right]^{2}}
$$

Como la velocidad de onda local $c(p)=c_{o}+a p$ crece desde $c_{o}$ hasta $c_{o}+a \mathcal{P}_{o}$ la curva se inclinará hacia la derecha porque los puntos de mayor presión también son los de mayor velocidad. Este comportamiento se muestra en la Figura 1.3. Llegará un momento en que la curva se haya inclinado tanto que empezará a ser multivaluada, aparece la onda de choque o ruptura de la onda como se comentó en la sección anterior.

La expresión (1.12) permite obtener el instante en que aparece la onda de choque. Sin embargo, aquí se abordará el problema de un modo diferente. Se parte de la inversa de la función (1.18), esto es:

$$
x(p, t)=\left(c_{o}+a p\right) t \pm \sqrt{\ln \frac{\mathcal{P}_{o}}{p}}, \quad 0 \leq p \leq \mathcal{P}_{o}
$$




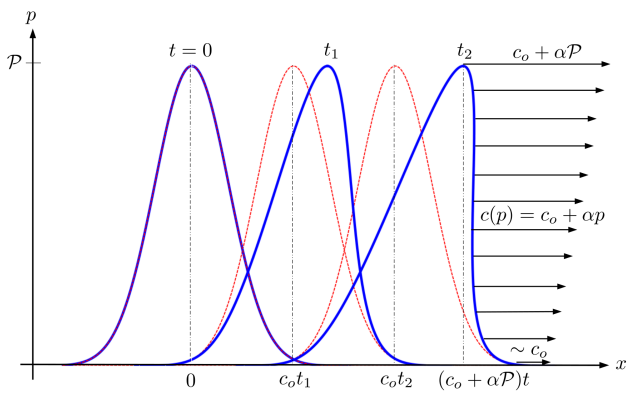

Figura 1.3: Propagación en régimen no lineal. El pulso se deforma debido a la velocidad de onda local $c(p)=c_{o}+a p$. La curva de color rojo corresponde al régimen lineal mostrado en la (Figura 1.2)

donde el signo + se corresponde con la rama derecha y el signo - con la rama izquierda respecto al máximo de la función. Es evidente que el choque ocurre en la rama derecha, por tanto, será suficiente considerar tan sólo el signo + en la función (1.19). Haciendo uso de la derivada de la función inversa (a $t$ constante) se obtiene:

$$
\frac{\partial p(x, t)}{\partial x}=\left[\frac{\partial x(p, t)}{\partial p}\right]^{-1}=\frac{2 p \sqrt{\ln \frac{\mathcal{P}_{o}}{p}}}{2 a p t \sqrt{\ln \frac{\mathcal{P}_{o}}{p}}-1}
$$

El choque ocurre cuando la derivada se hace infinita. Esto es, si:

$$
2 a p t \sqrt{\ln \frac{\mathcal{P}_{o}}{p}}-1=0 \Rightarrow t(p)=\frac{1}{2 a p \sqrt{\ln \frac{\mathcal{P}_{o}}{p}}}
$$

Para obtener los valores de presión y tiempo donde ocurre el choque $\left(p_{s h}\right.$ y $\left.t_{s h}\right)$ se calcula el mínimo absoluto de la función $t(p)$ dada por la ecuación (1.21). Este cálculo se puede realizar igualando la primera derivada a cero:

$$
\frac{d t(p)}{d p}=\frac{1-2 \ln \frac{\mathcal{P}_{o}}{p}}{4 p^{2} a\left[\ln \frac{\mathcal{P}_{o}}{p}\right]^{\frac{3}{2}}}=0 \quad \Longrightarrow \quad 1-2 \ln \frac{\mathcal{P}_{o}}{p}=0
$$

de donde, 


$$
p_{s h}=e^{-\frac{1}{2}} \mathcal{P}_{o} \simeq 0.607 \mathcal{P}_{o}
$$

Sustituyendo (1.23) en (1.21) resulta:

$$
t_{s h}=\frac{e^{\frac{1}{2}}}{\sqrt{2}} \frac{1}{a \mathcal{P}_{o}} \simeq \frac{1.166}{a \mathcal{P}_{o}}
$$

Queda por determinar la posición donde ocurre el choque. Esta posición se obtiene sustituyendo los resultados (1.23) y (1.24) en (1.19):

$$
x_{s h}=\frac{1}{\sqrt{2}}\left(2+e^{\frac{1}{2}} \frac{c_{o}}{a \mathcal{P}_{o}}\right)
$$

La Figura 1.4 muestra el perfil de onda en el instante del choque y en un tiempo posterior a él. Para graficar esta figura, se tomaron los siguientes valores numéricos:

$$
\mathcal{P}_{o}=1 \mathrm{MPa}, \quad c_{o}=1481.44 \mathrm{~m} / \mathrm{s}, \quad a=6.753 \cdot 10^{-6} \mathrm{~m}^{2} \mathrm{~s} / \mathrm{kg}
$$

Sustituyendo estos valores en (1.23), (1.24) y (1.25) se encuentra,

$$
p_{s h}=0.607 \mathrm{MPa}, \quad t_{s h}=0.173 \mathrm{~s}, \quad \mathrm{y} \quad x_{s h}=257.167 \mathrm{~m}
$$

Se observa que, para $t>t_{s h}$ la función deja de ser univaluada y la solución ya no es válida. Por lo tanto, el problema de Cauchy (1.13) es físicamente aceptable solamente si $t \leq 0.173 \mathrm{~s}$.
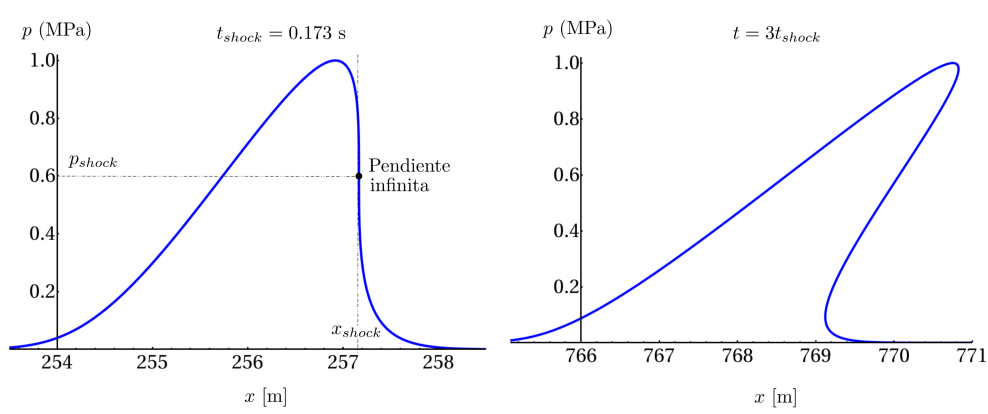

Figura 1.4: Para $t>t_{s h}$ la curva se hace tan pronunciada que es multivaluada 


\subsubsection{Propagación de una onda armónica con condiciones iniciales}

El ejemplo anterior, permitió mostrar la distorsión progresiva de un pulso gaussiano a lo largo de su propagación. El siguiente ejemplo, mostrará cómo se modifica el espectro espacial de una onda armónica en el transcurso del tiempo. Para ello, se considera el problema de Cauchy para la ecuación de Burgers con un perfil de onda senoidal de amplitud $\mathcal{P}_{o}$ y con la misma velocidad de onda local que en el caso anterior $c(p)=c_{o}+a p$ :

$$
\left\{\begin{array}{l}
\frac{\partial p(x, t)}{\partial t}+\left(c_{o}+a p\right) \frac{\partial p(x, t)}{\partial x}=0 \\
p(x, 0)=\mathcal{P}_{o} \sin k_{o} x
\end{array}\right.
$$

donde $k_{o}$ es una constante dada. En este caso, la solución de Riemann (1.4) toma la forma:

$$
\begin{aligned}
p(x, t) & =f[x-c(p) t] \\
& =\mathcal{P}_{o} \sin k_{o}[x-c(p) t]=\mathcal{P}_{o} \sin k_{o}\left(x-c_{o} t-a p t\right) \\
& =\mathcal{P}_{o} \sin \left(k_{o} x-\omega_{o} t-k_{o} a p t\right)
\end{aligned}
$$

$\mathrm{y} \omega_{o}=k_{o} c_{o}$.

Se usará la expresión (1.12) para obtener $t_{s h}$ y teniendo en cuenta que $c^{\prime}(p)=a$, resulta:

$$
t_{s h}=\frac{1}{a\left(-f^{\prime}(\xi)\right)_{\text {máx }}}
$$

Con la condición inicial dada en (1.26), se tiene $f(\xi)=\mathcal{P}_{o} \sin k_{o} \xi$, siendo su derivada $f^{\prime}(\xi)=k_{o} \mathcal{P}_{o} \cos k_{o} \xi$, resultando por tanto $\left(-f^{\prime}(\xi)\right)_{\text {máx }}=k_{o} \mathcal{P}_{o}$. De donde:

$$
t_{s h}=\frac{1}{k_{o} a \mathcal{P}_{o}}
$$

Este resultado permite escribir (1.27) como:

$$
p(x, t)=\mathcal{P}_{o} \sin \left[k_{o} x-\omega_{o} t-\frac{t}{t_{s h}} \frac{p(x, t)}{\mathcal{P}_{o}}\right]
$$


Dejando los detalles matemáticos para apartados posteriores, ahora simplemente se enuncia que: para $t<t_{s h}$ la solución de Riemman (1.30) admite un desarrollo en serie de Fourier en la forma:

$$
p(x, t)=\sum_{n=1}^{\infty} B_{n}(t) \sin \left[n\left(k_{o} x-\omega_{o} t\right)\right]
$$

donde los coeficientes $B_{n}(t)$ vienen dados por:

$$
B_{n}(t)=(-1)^{n-1} 2 \mathcal{P}_{o} \frac{t_{s h}}{n t} J_{n}\left(\frac{n t}{t_{s h}}\right)
$$

siendo $J_{n}$ la función de Bessel de primera especie y orden $n$. Este desarrollo es conocido como solución de Bessel-Fubini al problema de valor inicial (1.26).

La función (1.31) y los primeros coeficientes de Fourier (1.32) se grafican en la Figura 1.5 en tres instantes de tiempo. Para ello, se tomaron los siguientes valores numéricos:

$$
\mathcal{P}_{o}=1 \mathrm{MPa}, \quad c_{o}=1481.44 \mathrm{~m} / \mathrm{s}, \quad a=6.753 \cdot 10^{-6} \mathrm{~m}^{2} \mathrm{~s} / \mathrm{kg}, \quad k_{o}=425 \mathrm{rad} / \mathrm{m}
$$

resultando ser, según $(1.29), t_{s h}=349.15 \mu$ s.

En la Figura 1.5, la curva de color rojo muestra la solución lineal y la de color azul la no lineal. Se observa que, para $t=0$, se tiene en el perfil de onda inicial, una función senoidal de longitud de onda $\lambda=2 \pi / k_{o}$. En este instante, los coeficientes de Fourier son todos nulos excepto el primero, cuyo valor es igual a la amplitud de la función, esto es, $B_{1}=\mathcal{P}_{o}, B_{n}=0(n>1)$. En $t=\frac{1}{2} t_{s h}$ la función se distorsiona inclinándose hacia la derecha pero todavía no se alcanza el choque y la función mantiene una pendiente finita en todos sus puntos. Como consecuencia de esta deformación, el espectro de la función se modifica y los coeficientes $B_{n}$ empiezan a tomar valores distintos de cero. En este instante de tiempo, se observa que $B_{1}<\mathcal{P}_{o}$ puesto que el modo fundamental debe transferir energía al resto de los modos. En $t=t_{s h}$ aparece el choque y la función alcanza tangentes verticales en $p=0$ y distancias dadas por la expresión:

$$
x_{m}=\frac{(2 m-1) \pi}{k_{o}}+c_{o} t_{s h}, \quad m=0, \pm 1, \pm 2, \pm 3, \ldots
$$

Numéricamente: $x_{m}=0.614,2.096,3.577,5.059,6.540$ y $8.021 \mathrm{~cm}$ 

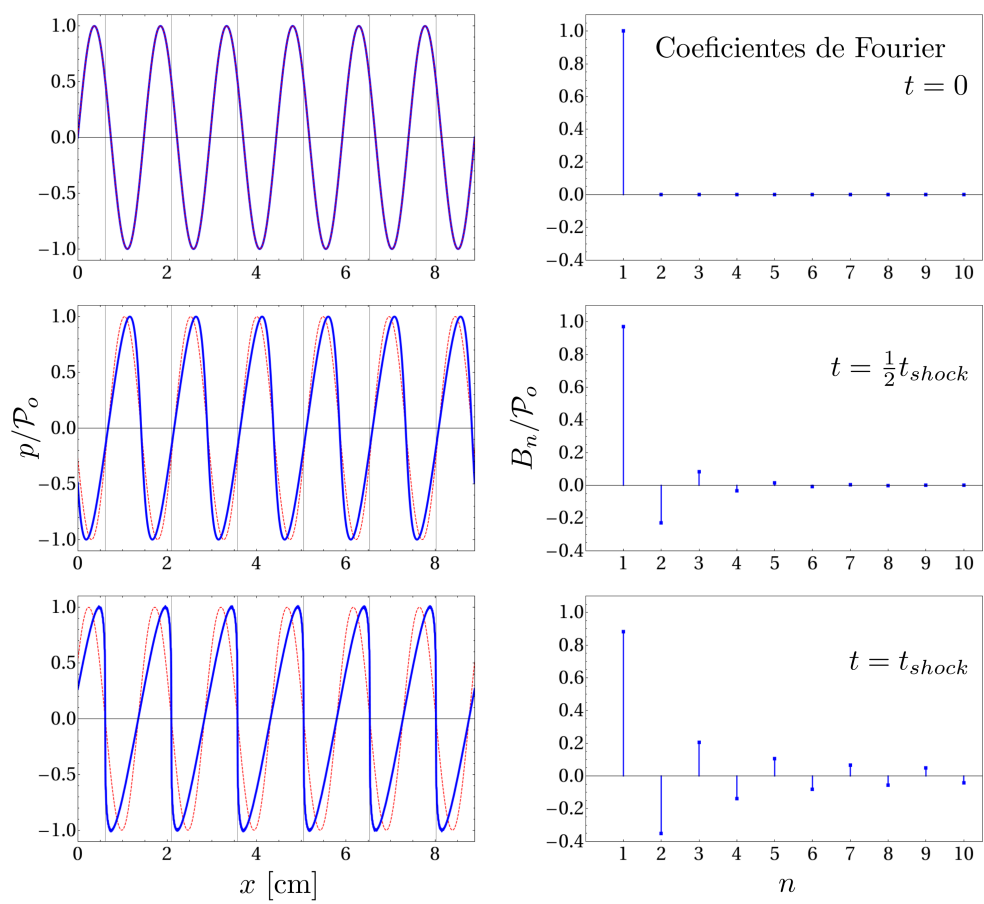

Figura 1.5: Deformación temporal de una onda senoidal $\left(t_{s h}=349.15 \mu \mathrm{s}\right)$

Por ser un problema de valor inicial, el espectro es espacial (número de onda) y cambia con el tiempo. Más adelante, se estudiará este mismo problema pero con condiciones de frontera, en cuyo caso, el espectro será temporal y cambiará con la distancia.

Este ejemplo junto con el anterior, han permitido cuantificar la distorsión y el cambio espectral de una onda no lineal. Aunque pudiera parecerlo, estos efectos no son un desventaja sino todo lo contrario. La riqueza espectral de las ondas no lineales son aprovechadas en multitud de aplicaciones prácticas, en particular, en el campo de las comunicaciones acústicas, tema central de esta tesis.

Si bien, la ecuación de Burgers ha sido apropiada como punto de partida para explicar los efectos no lineales, el modelo es relativamente sencillo y limitado. De hecho, la ecuación de Burgers es una versión simplificada uni-dimensional de la célebre ecuación de Westervelt [1], ampliamente utilizada en el campo de la acústica no lineal. Esta ecuación se obtiene a partir del modelo hidrodinámico bajo determinadas suposiciones y aproximaciones. Conocer estas aproximaciones es importante para poder acotar sus 
limitaciones y rango de validez. El objetivo del próximo capítulo, será deducir esta ecuación.

\subsection{Modelo hidrodinámico}

El modelo hidrodinámico, es el punto de partida para derivar las ecuaciones fundamentales de la acústica. Desafortunadamente, el conjunto completo de ecuaciones hidrodinámicas aún no se han resuelto, ni siquiera en el caso computacional (debido al gran número de operaciones requeridas y problemas de inestabilidades). Por ello, es necesario simplificar el modelo para poder obtener soluciones analíticas o numéricas cerradas.

Dependiendo del problema que se considere, se pueden usar varios modelos con diferentes grados de aproximación. Aquí se usará la ecuación hidrodinámica de continuidad y la ecuación de Euler.

- Ecuación de continuidad. Esta ecuación enuncia matemáticamente el principio de conservación de la masa y en forma diferencial se expresa como:

$$
\frac{\partial \rho}{\partial t}+\vec{\nabla} \cdot(\rho \vec{u})=0
$$

donde $\rho=\rho(\vec{r}, t)$ es la densidad y $\vec{u}=\vec{u}(\vec{r}, t)$ el vector velocidad asociado al movimiento de las partículas del fluido. Esta ecuación es no lineal al contener un término producto.

- Ecuación de Euler. Esta ecuación se deriva de la ley de conservación de la cantidad de movimiento o segunda ley de Newton para fluidos no viscosos. En ausencia de fuerzas externas, la ecuación de Euler toma la forma:

$$
\rho\left[\frac{\partial \vec{u}}{\partial t}+(\vec{u} \cdot \vec{\nabla}) \vec{u}\right]=-\vec{\nabla} p
$$

donde $p=p(\vec{r}, t)$ es la presión. El término $(\vec{u} \cdot \nabla) \vec{u}$ se llama término de aceleración convectiva o de transporte. Es un término no lineal y su no linealidad, en principio, puede desarrollar singularidades en la solución de la ecuación.

Usando las identidades vectoriales: 


$$
\begin{aligned}
& \vec{\nabla} \cdot(\rho \vec{u})=\vec{u} \cdot \vec{\nabla} \rho+\rho \vec{\nabla} \cdot \vec{u} \\
& (\vec{u} \cdot \vec{\nabla}) \vec{u}=\frac{1}{2} \vec{\nabla} u^{2}-\vec{u} \times(\vec{\nabla} \times \vec{u})
\end{aligned}
$$

(donde $u^{2}=\vec{u} \cdot \vec{u}$ ) se puede escribir la ecuación de continuidad (1.34) y la ecuación de Euler (1.35) de una forma más apropiada como:

$$
\begin{aligned}
& \frac{\partial \rho}{\partial t}+\vec{u} \cdot \vec{\nabla} \rho+\rho \nabla \cdot \vec{u}=0 \\
& \rho\left[d \frac{\partial \vec{u}}{\partial t}+\frac{1}{2} \vec{\nabla} u^{2}-\vec{u} \times(\vec{\nabla} \times \vec{u})\right]=-\vec{\nabla} p
\end{aligned}
$$

Las variables que aparecen en estas ecuaciones son cantidades absolutas de presión, densidad y velocidad. Suponiendo el fluido inicialmente en equilibrio, la propagación de una onda acústica producirá una perturbación, es decir, una desviación respecto a los valores en el equilibrio. Así, las cantidades absolutas pueden descomponerse como suma de dos términos:

$$
\begin{aligned}
& p(\vec{r}, t)=p_{o}+p^{\prime}(\vec{r}, t) \\
& \rho((\vec{r}, t))=\rho_{o}+\rho^{\prime}(\vec{r}, t) \\
& \vec{u}(\vec{r}, t)=\vec{u}_{o}+\vec{u}^{\prime}(\vec{r}, t)
\end{aligned}
$$

El subíndice cero, denota valores en el equilibrio y, el índice prima, denota valores perturbados. Por ejemplo, $p^{\prime}$ representa la desviación que sufre la presión respecto al estado de equilibrio $p_{o}$, esta perturbación se conoce como presión acústica.

En lo que sigue, se supone el medio homogéneo para el cual, las cantidades $\rho_{o}$ y $p_{o}$ son constantes (independientes de la posición y del tiempo). También se supone, que la velocidad del fluido en equilibrio es nula $\vec{u}_{o}=0$ y además, si se desprecia los efectos de bordes, se puede suponer que el campo vectorial de velocidad acústica $\vec{u}^{\prime}$ es irrotacional $\vec{\nabla} \times \vec{u}^{\prime}=0$. Bajo estos supuestos, si se sustituye (1.39) en (1.38) se obtiene:

$$
\begin{aligned}
& \frac{\partial \rho^{\prime}}{\partial t}+\rho_{o} \vec{\nabla} \cdot \vec{u}^{\prime}=-\vec{u}^{\prime} \cdot \vec{\nabla} \rho^{\prime}-\rho^{\prime} \vec{\nabla} \cdot \vec{u}^{\prime} \\
& \rho_{o} \frac{\partial \vec{u}^{\prime}}{\partial t}+\vec{\nabla} p^{\prime}=-\rho^{\prime} \frac{\partial \vec{u}^{\prime}}{\partial t}-\frac{1}{2} \rho_{o} \vec{\nabla} u^{\prime 2}-\frac{1}{2} \rho^{\prime} \vec{\nabla} u^{\prime 2}
\end{aligned}
$$


El objetivo, es resolver este sistema de ecuaciones en la variable $p^{\prime}$. Se supone que las fluctuaciones son relativamente pequeñas $p^{\prime} / p_{o}, \rho^{\prime} / \rho, u^{\prime} / u_{o}<<1$. Esto permitirá despreciar términos a partir de un cierto orden de exactitud. Usando el método introducido por Blackstock [2], se puede clasificar el orden de un determinado término según el siguiente criterio:

- Términos de primer orden. Se consideran términos de primer orden aquellos que son lineales respecto a la perturbación. Por ejemplo, son términos de primer orden todos los colocados a la izquierda de la igualdad en las ecuaciones (1.40) y (1.41).

- Términos de segundo orden. Los términos de segundo orden, son todos los términos cuadráticos que aparecen en los productos cruzados entre magnitudes perturbadas. Por ejemplo, son términos de segundo orden todos los colocados a la derecha de la igualdad en las ecuaciones (1.40) y (1.41) excepto el término $\rho^{\prime} \vec{\nabla} u^{\prime 2}$ que es de mayor orden.

- Términos de más alto orden. Todos los restantes.

Adicionalmente, se hará uso del llamado corolario de sustitución. Según este corolario se puede sustituir, cuando sea necesario, aproximaciones de primer orden en expresiones de segundo o más alto orden. En particular, si en una expresión de segundo orden se introducen aproximaciones de primer orden, el resultado es una aproximación de tercer orden y el corolario queda justificado.

Por otra parte, es importante observar que el sistema de ecuaciones (1.40) y (1.41), es un sistema formado por una ecuación escalar y otra vectorial. Por lo tanto, es un sistema indeterminado, pues contiene cuatro ecuaciones y cinco incógnitas $\left(p^{\prime}, \rho^{\prime}, u_{x}^{\prime}\right.$, $u_{y}^{\prime}$ y $u_{z}^{\prime}$ ). Para que el sistema sea completo, es necesario añadir una ecuación más. Esta tercera ecuación, es la ecuación de estado. Suponiendo el fluido barotrópico, la presión depende tan sólo de la densidad y la ecuación de estado toma la forma $p=p(\rho)$. Si se desarrolla esta función en serie de Taylor en torno al valor de equilibrio $\rho_{o}$ y se desprecian los términos de orden superior a dos, se obtiene para la presión acústica la siguiente ecuación de estado (ver apartado A.1 para los detalles matemáticos),

$$
p^{\prime}=c_{o}^{2} \rho^{\prime}+\frac{c_{o}^{2}}{\rho_{o}} \frac{B}{2 A} \rho^{\prime 2}
$$

donde $B / A$ es una cantidad adimensional conocida como $p$ arámetro de no linealidad, se mide experimentalmente y sus valores son tabulados. Por ejemplo, un valor típico para el agua es $B / A=5.2$. 


\subsubsection{Aproximación de $1^{\circ}$ orden (Acústica lineal)}

Para señales de pequeña amplitud, el desplazamiento característico de las magnitudes del fluido respecto a sus valores de equilibrio, es muy pequeño. Esta condición define la hipótesis de la acústica lineal. Bajo esta hipótesis, con un buen grado de aproximación, se puede despreciar en las ecuaciones (1.40), (1.41) y (1.42), los términos de orden mayor que uno. En estas condiciones, la ecuación de continuidad, la ecuación de Euler y la ecuación de estado se reducen a:

$$
\left\{\begin{array}{l}
\frac{\partial \rho^{\prime}}{\partial t}+\rho_{o} \vec{\nabla} \cdot \vec{u}^{\prime}=0 \\
\rho_{o} \frac{\partial \vec{u}^{\prime}}{\partial t}+\vec{\nabla} p^{\prime}=0 \\
p^{\prime}=c_{o}^{2} \rho^{\prime}
\end{array}\right.
$$

Estas tres ecuaciones a primer orden son la base matemática de la acústica lineal. El sistema está compuesto de dos ecuaciones diferenciales acopladas (1.43a)-(1.43b) y una ecuación algebraica (1.43c) que las liga linealmente. Esto permite despejar una de las tres variables para obtener un ecuación desacoplada. Despejando $p^{\prime}$ se obtiene la ecuación de onda lineal para la presión acústica:

$$
\nabla^{2} p^{\prime}-\frac{1}{c_{o}^{2}} \frac{\partial^{2} p^{\prime}}{\partial t^{2}}=0
$$

Para ondas armónicas planas $\psi(\vec{r}, t)=\psi_{o} e^{j(\vec{k} \cdot \vec{r}-\omega t)}$ es fácil demostrar, que se cumple la siguiente relación entre las cantidades acústicas $\vec{u}^{\prime}$ y $p^{\prime}$,

$$
\frac{p^{\prime}}{u^{\prime}}=\rho_{o} c_{o}
$$

Esta relación recibe el nombre de impedancia acústica y es una medida de la resistencia que opone un medio a la propagación de ondas acústicas. 


\subsubsection{Aproximación de $2^{\circ}$ orden (Acústica no lineal)}

En señales de gran amplitud, el modelo lineal deja de ser apropiado y será necesario considerar términos de mayor orden. La mayor parte de las aplicaciones de interés, pueden ser formuladas con un buen grado de exactitud bajo una aproximación de segundo orden.

En las ecuaciones (1.40), (1.41) y (1.42), todos los términos involucrados son de segundo orden excepto el término $\rho^{\prime} \vec{\nabla} u^{\prime 2}$ que es de tercer orden. Así, para una aproximación de segundo orden se tiene:

$$
\begin{aligned}
& \frac{\partial \rho^{\prime}}{\partial t}+\rho_{o} \vec{\nabla} \cdot \vec{u}^{\prime}=-\vec{u}^{\prime} \cdot \vec{\nabla} \rho^{\prime}-\rho^{\prime} \vec{\nabla} \cdot \vec{u}^{\prime} \\
& \rho_{o} \frac{\partial \vec{u}^{\prime}}{\partial t}+\vec{\nabla} p^{\prime}=-\rho^{\prime} \frac{\partial \vec{u}^{\prime}}{\partial t}-\frac{1}{2} \rho_{o} \vec{\nabla} u^{\prime 2} \\
& p^{\prime}=c_{o}^{2} \rho^{\prime}+\frac{c_{o}^{2}}{\rho_{o}} \frac{B}{2 A} \rho^{\prime 2}
\end{aligned}
$$

A continuación, es necesario hacer uso del corolario de sustitución enunciado anteriormente. Se puede usar las ecuaciones del modelo lineal a primer orden (1.43a), (1.43b) y (1.43c) para aproximar los términos de segundo orden en las ecuaciones (1.46), (1.47) y (1.48). Después de un poco de álgebra el resultado es:

$$
\left\{\begin{array}{l}
\frac{\partial \rho^{\prime}}{\partial t}+\rho_{o} \vec{\nabla} \cdot \vec{u}^{\prime}=\frac{1}{\rho_{o} c_{o}^{4}} \frac{\partial p^{2}}{\partial t}+\frac{1}{c_{o}^{2}} \frac{\partial \mathcal{L}}{\partial t} \\
\rho_{o} \frac{\partial \vec{u}^{\prime}}{\partial t}+\vec{\nabla} p^{\prime}=-\vec{\nabla} \mathcal{L} \\
p^{\prime}=c_{o}^{2} \rho^{\prime}+\frac{1}{\rho_{o} c_{o}^{2}} \frac{B}{2 A} p^{\prime 2}
\end{array}\right.
$$

donde se define,

$$
\mathcal{L} \equiv \frac{1}{2}\left(\rho_{o} u^{\prime 2}-\frac{p^{\prime 2}}{c_{o}^{2} \rho_{o}}\right)
$$

Esta cantidad recibe el nombre de densidad lagrangiana de segundo orden. Según la relación lineal (1.45) se observa que, para ondas armónicas planas, $\mathcal{L}=0$. 
Las tres ecuaciones (1.49a), (1.49b) y (1.49c), son las ecuaciones básicas de la acústica no lineal de segundo orden en fluidos perfectos (los que no sufren efectos viscosos) y sin conductividad térmica. Como en el caso lineal, ahora se despeja $p^{\prime}$ y así obtener la ecuación de onda para la presión acústica:

$$
\nabla^{2} p^{\prime}-\frac{1}{c_{o}^{2}} \frac{\partial^{2} p^{\prime}}{\partial t^{2}}=-\frac{\beta}{\rho_{o} c_{o}^{4}} \frac{\partial^{2} p^{\prime 2}}{\partial t^{2}}-\left(\nabla^{2} \mathcal{L}+\frac{1}{c_{o}^{2}} \frac{\partial^{2} \mathcal{L}}{\partial t^{2}}\right)
$$

definiendo,

$$
\beta=1+\frac{B}{2 A}
$$

Esta cantidad recibe el nombre de coeficiente de no linealidad.

\subsection{Ecuación de Westervelt y Burgers}

La ecuación de Westervelt (1963) [1], quizás sea la ecuación más conocida en el campo de la acústica no lineal, cuyo estudio se basó en el "scattering of sound by sound". Esta ecuación, se obtiene haciendo la aproximación $\mathcal{L}=0$ en la ecuación de onda (1.51). La densidad lagrangiana $\mathcal{L}$ puede omitirse cuando los efectos no lineales acumulativos, dominan sobre los efectos no lineales locales [3]. Haciendo esta aproximación, se obtiene la deseada ecuación de Westervelt:

$$
\nabla^{2} p^{\prime}-\frac{1}{c_{o}^{2}} \frac{\partial^{2} p^{\prime}}{\partial t^{2}}=-\frac{\beta}{\rho_{o} c_{o}^{4}} \frac{\partial^{2} p^{\prime 2}}{\partial t^{2}}
$$

La ecuación de Westervelt uni-dimensional:

$$
\frac{\partial^{2} p^{\prime}}{\partial x^{2}}-\frac{1}{c_{o}^{2}} \frac{\partial^{2} p^{\prime}}{\partial t^{2}}=-\frac{\beta}{\rho_{o} c_{o}^{4}} \frac{\partial^{2} p^{2}}{\partial t^{2}}
$$

puede ser simplificada introduciendo un cambio de variable. Se toma como dirección de propagación el eje $x$ y se definen las siguientes variables,

$$
x_{1}=\epsilon x, \quad \tau=t-\frac{x}{c_{o}}
$$


la variable $\tau$ se denomina tiempo de retardo y $\epsilon$ se tratará como un término de primer orden. Haciendo uso de la regla de la cadena, se comprueba que con este cambio de variables se pueden expresar las derivadas parciales en la forma:

$$
\frac{\partial p^{\prime}(x, t)}{\partial x}=\epsilon \frac{\partial p^{\prime}\left(x_{1}, \tau\right)}{\partial x_{1}}-\frac{1}{c_{o}} \frac{\partial p^{\prime}\left(x_{1}, \tau\right)}{\partial \tau}, \quad \frac{\partial p^{\prime}(x, t)}{\partial t}=\frac{\partial p^{\prime}\left(x_{1}, \tau\right)}{\partial \tau}
$$

Esto permite reescribir la ecuación uni-dimensional de Westervelt (1.54) como:

$$
\epsilon^{2} \frac{\partial^{2} p^{\prime}}{\partial x_{1}^{2}}-\frac{2 \epsilon}{c_{o}} \frac{\partial^{2} p^{\prime}}{\partial x_{1} \partial \tau}=-\frac{\beta}{\rho_{o} c_{o}^{4}} \frac{\partial^{2} p^{2}}{\partial \tau^{2}}
$$

Se observa que el primer término a la derecha de la igualdad es de tercer orden, por lo tanto, se podrá despreciar en una aproximación de segundo orden. Lo que permite escribir,

$$
\frac{\partial}{\partial \tau}\left(\frac{2 \epsilon}{c_{o}} \frac{\partial^{2} p^{\prime}}{\partial x_{1}}\right)=\frac{\partial}{\partial \tau}\left(\frac{\beta}{\rho_{o} c_{o}^{4}} \frac{\partial p^{2}}{\partial \tau}\right)
$$

Integrando en $\tau$ y teniendo en cuenta que $\partial p^{2} / \partial \tau=2 p^{\prime} \partial p^{\prime} / \partial \tau$ resulta:

$$
\epsilon \frac{\partial p^{\prime}}{\partial x_{1}}=\frac{\beta p^{\prime}}{\rho_{o} c_{o}^{3}} \frac{\partial p^{\prime}}{\partial \tau}
$$

Deshaciendo el cambio de variable espacial, se obtiene la ecuación de Burgers en función del tiempo de retardo $\tau$ :

$$
\frac{\partial p^{\prime}}{\partial x}=\frac{\beta p^{\prime}}{\rho_{o} c_{o}^{3}} \frac{\partial p^{\prime}}{\partial \tau}
$$

En función de $x$ y $t$ la ecuación de Burgers se expresa como:

$$
\frac{\partial p^{\prime}}{\partial t}+c\left(p^{\prime}\right) \frac{\partial p^{\prime}}{\partial x}=0
$$

con 


$$
\begin{aligned}
c\left(p^{\prime}\right) & =c_{o}\left(1-\frac{\beta p^{\prime}}{\rho_{o} c_{o}^{2}}\right)^{-1} \\
& =c_{o}\left(1-\frac{a p^{\prime}}{c_{o}}\right)^{-1} \simeq c_{o}+a p^{\prime}, \quad a=\beta / \rho_{o} c_{o}
\end{aligned}
$$

donde se usó el desarrollo $(1-x)^{-1} \simeq 1+x+x^{2}+\cdots$. Se observa que, (1.63) es la velocidad de onda local que se usó en los problemas de Cauchy (1.13) y (1.26).

Hasta ahora, no se ha tenido en cuenta los efectos de la viscosidad. Sin embargo, los fluidos que no sufren efectos viscosos no dejan de ser un tanto irrealistas, pues todo fluido, en mayor o menor medida, poseen un cierto grado de viscosidad. Siguiendo el mismo esquema de las secciones anteriores, en el apéndice B, se obtienen las ecuaciones de Westervelt y Burgers en medios viscosos (un análisis completo también puede encontrarse en [3]). Estas ecuaciones toman la forma:

$$
\begin{gathered}
\nabla^{2} p^{\prime}-\frac{1}{c_{o}^{2}} \frac{\partial^{2} p^{\prime}}{\partial t^{2}}=-\frac{\beta}{\rho_{o} c_{o}^{4}} \frac{\partial^{2} p^{2}}{\partial t^{2}}-\frac{\delta}{c_{o}^{4}} \frac{\partial^{3} p^{\prime}}{\partial t^{3}} \\
\frac{\partial p^{\prime}}{\partial x}=\frac{\beta p^{\prime}}{\rho_{o} c_{o}^{3}} \frac{\partial p^{\prime}}{\partial \tau}+\frac{\delta}{2 c_{o}^{3}} \frac{\partial^{2} p^{\prime}}{\partial \tau^{2}}
\end{gathered}
$$

donde $\delta>0$ es la difusividad del sonido definida como:

$$
\delta=\frac{1}{\rho_{o}}\left(\frac{4}{3} \mu+\mu_{b}\right)
$$

siendo $\mu$ la viscosidad dinámica o absoluta y $\mu_{b}$ la viscosidad de volumen o segundo coeficiente de viscosidad.

En dicho apéndice también se obtuvo la ecuación de onda lineal para fluidos viscosos que aquí se repite por comodidad:

$$
\frac{\partial^{2} p^{\prime}}{\partial t^{2}}-c_{o}^{2} \nabla^{2} p^{\prime}=\delta \frac{\partial}{\partial t}\left(\nabla^{2} p^{\prime}\right)
$$

En los próximos apartados se hará uso del llamado coeficiente de atenuación $\alpha_{o}$ cuyo significado es el siguiente. Sin pérdida de generalidad, se limitará al caso unidimensional, para el cual la ecuación (1.67) toma la forma: 


$$
\frac{\partial^{2} p^{\prime}}{\partial t^{2}}-c_{o}^{2} \frac{\partial^{2} p^{\prime}}{\partial x^{2}}=\delta \frac{\partial}{\partial t}\left(\frac{\partial^{2} p^{\prime}}{\partial x^{2}}\right)
$$

Se buscan soluciones del tipo ondas planas:

$$
p^{\prime}(x, t)=\mathcal{P}_{o} e^{j\left(k_{c} x-\omega_{o} t\right)}
$$

Sustituyendo (1.69) en (1.68) se obtiene la relación de dispersión compleja:

$$
k_{c}=\frac{\omega_{o}}{c_{o}}\left(1-j \frac{\omega_{o} \delta}{c_{o}^{2}}\right)^{-\frac{1}{2}} \simeq \frac{\omega_{o}}{c_{o}}\left(1+j \frac{\delta \omega_{o}}{2 c_{o}^{2}}\right)
$$

Pudiendo escribir:

$$
k_{c}=\frac{\omega_{o}}{c_{o}}+j \frac{\delta \omega_{o}^{2}}{2 c_{o}^{3}}=k_{o}+j \alpha_{o}
$$

con $k_{o}=\omega_{o} / c_{o}$ el número de onda y $\alpha_{o}$ el coeficiente de atenuación en la frecuencia $\omega_{o}$ :

$$
\alpha_{o}=\frac{\delta \omega_{o}^{2}}{2 c_{o}^{3}}
$$

El inverso de $\alpha_{o}$ se denomina longitud de absorción y se denota con el símbolo $L_{a}$, esto es:

$$
L_{a}=\frac{1}{\alpha_{o}}
$$

Sustituyendo (1.71) en (1.69) y tomando la parte imaginaria, se obtiene la forma de una onda lineal atenuada, característica de un medio con pérdidas o disipativo:

$$
p^{\prime}(x, t)=\mathcal{P}_{o} e^{-\alpha_{o} x} \sin \omega_{o}\left(t-\frac{x}{c_{o}}\right)
$$

Se observa que, cuando $x=L_{a}$ la amplitud de la señal se ha atenuado en un factor $\mathcal{P}_{o} / e$. También se observa de (1.72) que el coeficiente de atenuación $\alpha_{o}$ depende del 
cuadrado de la frecuencia. Por esta razón, las ondas de mayor frecuencia se disiparán con mayor rapidez que las ondas de más baja frecuencia.

\subsection{Problemas de contorno}

La ecuación de Burgers es ampliamente usada por ser un modelo sencillo en el que se observa la formación de singularidades de choque en tiempos finitos. La formación de estas singularidades, es uno de los fenómenos que mejor distinguen a las ecuaciones no lineales de las lineales.

En las secciones 1.1.1 y 1.1.2, se resolvieron dos problemas de condiciones iniciales para la ecuación de Burgers (1.61). Si bien, estos problemas tienen gran interés conceptual y práctico, no se adaptan a las condiciones experimentales de esta tesis, en donde el fluido es excitado con un transductor suponiendo condiciones iniciales nulas. Por ello, son de mayor interés los problemas de contorno donde se supone una frontera (la superficie del transductor) que vibra según una señal prefija. Dichos problemas de contorno para la ecuación de Burgers no viscosa (1.61) se expresan como:

$$
\left\{\begin{array}{l}
\frac{\partial p^{\prime}(x, t)}{\partial t}+c\left(p^{\prime}\right) \frac{\partial p^{\prime}(x, t)}{\partial x}=0 \\
p^{\prime}(0, t)=f(t)
\end{array}\right.
$$

donde la frontera se supone en $x=0$ y la velocidad de onda local $c\left(p^{\prime}\right)$ está dada por (1.62). Para problemas de contorno, la solución de Riemann en la forma (1.4) no es conveniente, y se usará la siguiente expresión equivalente:

$$
p^{\prime}(x, t)=f\left[t-\frac{x}{c\left(p^{\prime}\right)}\right]
$$

Sustituyendo (1.62) en (1.76) se puede escribir:

$$
p^{\prime}(x, t)=f\left[t-\frac{x}{c_{o}}+\frac{\beta p^{\prime}}{\rho_{o} c_{o}^{3}} x\right]
$$

Por otra parte, para calcular la aparición del choque en este tipo de problemas, la ecuación (1.12) no es apropiada. En este tipo de problemas, la distancia donde aparece la onda de choque se obtienen a partir de la expresión (ver apartado A.2 para los detalles matemáticos): 


$$
x_{s h}=\frac{\rho_{o} c_{o}^{3}}{\beta f_{\max }^{\prime}(\zeta)}
$$

En las siguientes secciones, se resolverá este problema de contorno con diferentes excitaciones y se estudiará, cómo evoluciona su espectro a lo largo de la distancia de propagación. La Figura 1.6, muestra esquemáticamente esta situación. Estos problemas serán la base teórica del desarrollo experimental de esta tesis.

$$
x=0
$$

Transductor
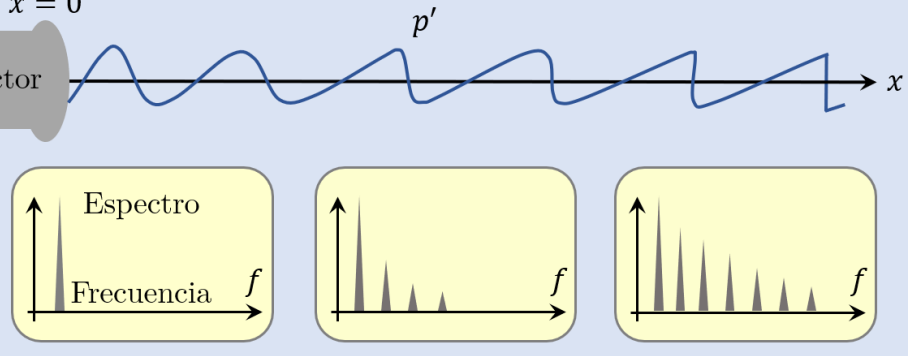

Figura 1.6: Propagación espacial de una señal armónica en regimen no lineal

\subsubsection{Excitación armónica mono-frecuencia en un medio no viscoso}

Solución de Riemann y Fubini (1935). En una excitación armónica mono-frecuencia de amplitud $\mathcal{P}_{o}$ y frecuencia $\omega_{o}$, el problema de contorno para la ecuación de Burgers no viscosa (1.75) se expresa como:

$$
\left\{\begin{array}{l}
\frac{\partial p^{\prime}(x, t)}{\partial t}+c\left(p^{\prime}\right) \frac{\partial p^{\prime}(x, t)}{\partial x}=0 \\
p^{\prime}(0, t)=\mathcal{P}_{o} \sin \omega_{o} t
\end{array}\right.
$$

En este caso $f(t)=\mathcal{P}_{o} \sin \omega_{o} t$ y según (1.77) la solución de Riemann toma la forma:

$$
p^{\prime}(x, t)=\mathcal{P}_{o} \sin \omega_{o}\left[t-\frac{x}{c_{o}}+\frac{\beta p^{\prime}}{\rho_{o} c_{o}^{3}} x\right]=\mathcal{P}_{o} \sin \left(\omega_{o} t-k_{o} x+\frac{\omega_{o} \beta p^{\prime}}{\rho_{o} c_{o}^{3}} x\right)
$$

donde se define $k_{o}=\omega_{o} / c_{o}$. Usando (1.78), se puede calcular la aparición del choque teniendo en cuenta que $f(\zeta)=\mathcal{P}_{o} \sin \omega_{o} \zeta$, su derivada resulta ser $f^{\prime}(\zeta)=\omega_{o} \mathcal{P}_{o} \cos \omega_{o} \zeta$, por tanto, $f_{\max }^{\prime}(\zeta)=\omega_{o} \mathcal{P}_{o}$. Usando el símbolos $\tilde{x}$ para denotar la distancia de choque en el caso de funciones mono-frecuencia, se tiene: 


$$
\tilde{x}_{s h}=\frac{\rho_{o} c_{o}^{3}}{\omega_{o} \beta \mathcal{P}_{o}}
$$

Este resultado permite expresar (1.79) en la forma:

$$
p^{\prime}(x, t)=\mathcal{P}_{o} \sin \left[\omega_{o} t-k_{o} x+\frac{x}{\tilde{x}_{s h}} \frac{p^{\prime}(x, t)}{\mathcal{P}_{o}}\right]
$$

Para $x \leq \tilde{x}_{s h}$ la solución de Riemann (1.81) admite un desarrollo en serie de Fourier conocido como solución Bessel-Fubini (ver apartado A.3):

$$
p^{\prime}(x, t)=\sum_{n=1}^{\infty} B_{n}(x) \sin \left[n \omega_{o}\left(t-\frac{x}{c_{o}}\right)\right]
$$

Donde los coeficientes $B_{n}(x)$ vienen dados por:

$$
B_{n}(x)=2 \mathcal{P}_{o} \frac{\tilde{x}_{s h}}{n x} J_{n}\left(\frac{n x}{\tilde{x}_{s h}}\right)
$$

siendo $J_{n}$ la función de Bessel de primera especie y orden $n$.

La función (1.82) es graficada en la Figura 1.7 con $t=0$ y $0 \leq x \leq \tilde{x}_{s h}$. Para ello se tomaron los siguientes valores numéricos:

$$
\mathcal{P}_{o}=1 \mathrm{MPa}, \quad c_{o}=1481.44 \mathrm{~m} / \mathrm{s}, \quad \rho_{o}=999.6 \mathrm{~kg} / \mathrm{m}^{3}, \quad \beta=10, \quad f_{o}=0.1 \mathrm{MHz}
$$

En la Figura 1.7, se observa cómo la onda se deforma progresivamente conforme se aleja de la fuente situada en $x=0$.

En la Figura 1.8, se vuelve a graficar la solución (1.82) pero en este caso mostrando la dependencia temporal en tres planos de observación. También se muestran los primeros coeficientes de Fourier $B_{n}$ dados por (1.83).

En la gráfica se observa que en $x=0$, se tiene en el perfil de la señal dada por la fuente, una función senoidal de periodo $T_{o}=2 \pi / \omega_{o}$. En esta posición, los coeficientes de Fourier son todos nulos excepto el primero, cuyo valor, es igual a la amplitud de la función, esto es, $B_{1}=\mathcal{P}_{o}, B_{n}=0(n>1)$. En $x=\frac{1}{2} \tilde{x}_{s h}$ la función está distorsionada, inclinada hacia la izquierda pero todavía no alcanza el choque y la función mantiene una pendiente finita en todos sus puntos. Como consecuencia de esta deformación, 

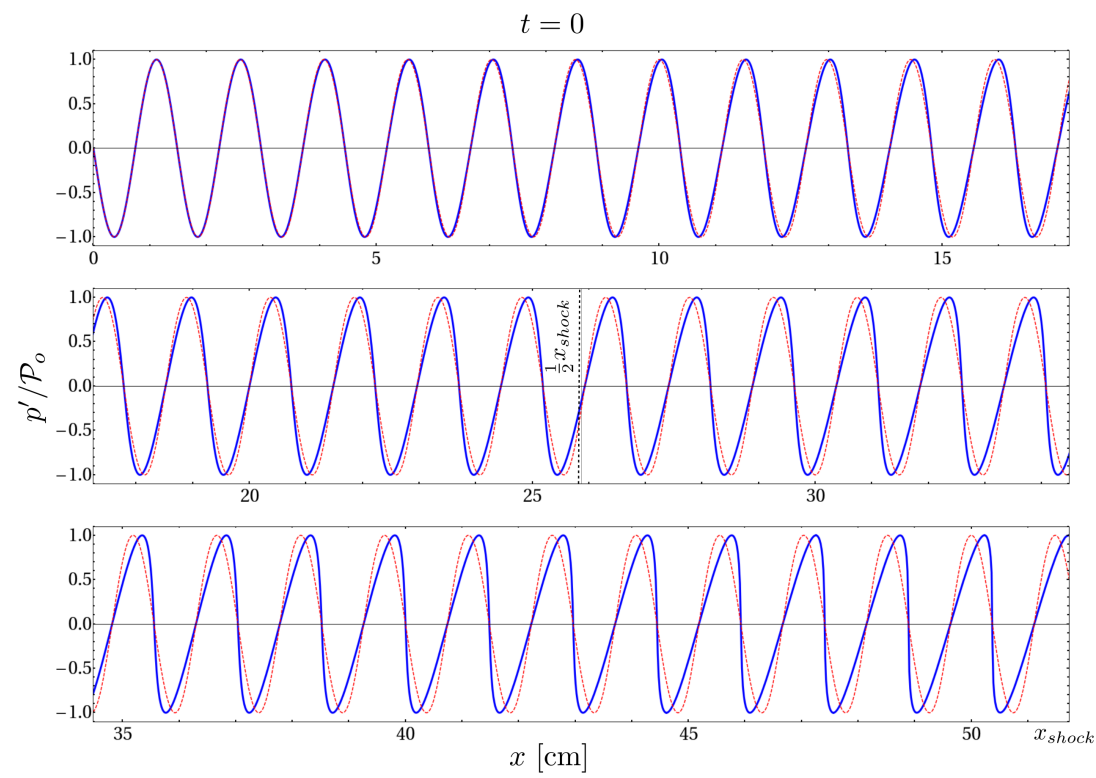

Figura 1.7: Deformación espacial de una onda armónica mono-frecuencial $\left(\tilde{x}_{s h}=51.72 \mathrm{~cm}\right)$

el espectro de la función se modifica y los coeficientes $B_{n}$ empiezan a tomar valores distintos de cero. En esta posición, se observa que $B_{1}<\mathcal{P}_{o}$ puesto que el modo fundamental debe transferir energía al resto de los modos. En $x=\tilde{x}_{s h}$ la función alcanza tangentes verticales en $p^{\prime}=0$ y en tiempos dados por la expresión:

$$
t_{m}=m T_{o}+\frac{\tilde{x}_{s h}}{c_{o}}, \quad m=0, \pm 1, \pm 2, \pm 3, \ldots
$$

Numéricamente: $t_{m}=9.15198,19.152,29.152 \mu \mathrm{s}$.

Se tiene que en el plano de observación $x=\tilde{x}_{s h}$, el primer choque se observa después de un tiempo $t=t_{0}$ (esto es $m=0$ ). Si se define $\tilde{t}_{s h}=t_{0}$ se obtiene la relación:

$$
\tilde{x}_{s h}=c_{o} \tilde{t}_{s h}
$$

Así, conocido $\tilde{x}_{s h}$, se puede determinar fácilmente el tiempo que ha de transcurrir para observar la primera tangente vertical en $x=\tilde{x}_{s h}$. La velocidad con que esto ocurre es la velocidad del sonido de pequeña amplitud $\left(c_{o}\right)$. 

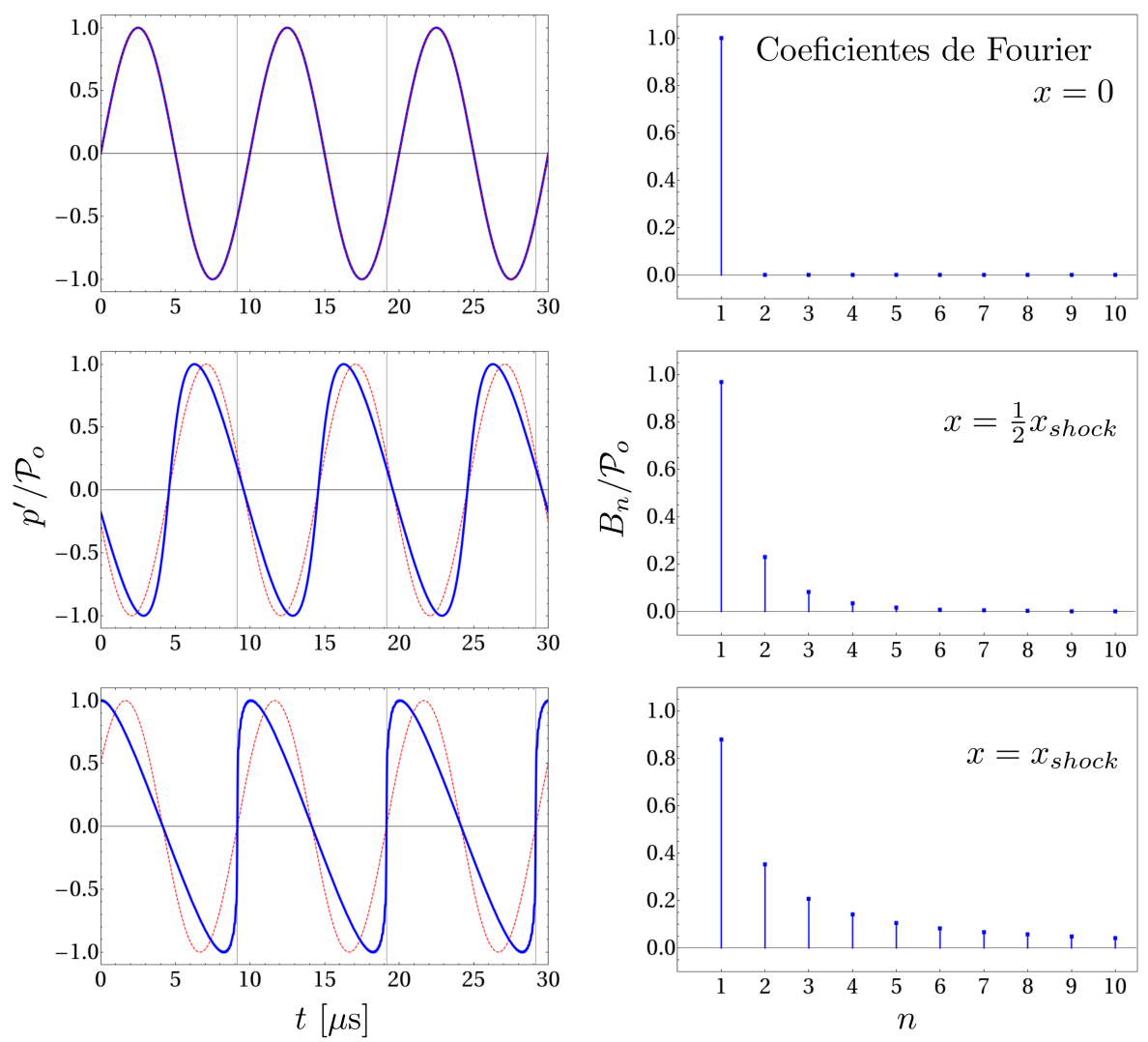

Figura 1.8: Deformación temporal de una onda senoidal $\left(\tilde{x}_{s h}=51.72 \mathrm{~cm}\right)$. La curva de color rojo muestra la solución lineal

En la Figura 1.9 se grafica $B_{n}(x)$ (ecuación (1.83)) como función de $x$. Esta gráfica, permite visualizar cómo evolucionan las distintas componentes espectrales con la distancia. Se observa que el decrecimiento del armónico fundamental $B_{1}$ va acompañado de un crecimiento en las restantes componentes espectrales, existiendo un intercambio de energía desde el modo fundamental al resto de los modos. 


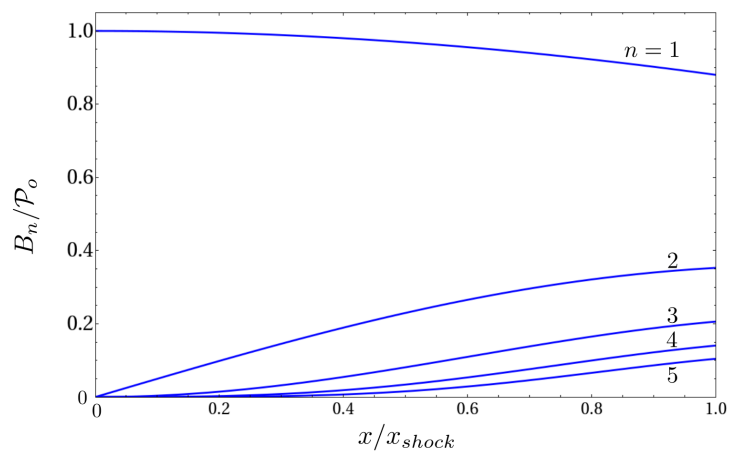

Figura 1.9: Evolución de los primeros cinco coeficientes $B_{n}(x)$ como función de la distancia

\subsubsection{Excitación armónica bi-frecuencia en un medio no viscoso}

Solución de Riemann y Fenlon (1972). Se considera ahora, el problema de contorno para la ecuación de Burgers con una excitación bi-armónica de amplitudes $\mathcal{P}_{a}$ y $\mathcal{P}_{b}$ y frecuencias $\omega_{a}$ y $\omega_{b}$,

$$
\left\{\begin{array}{l}
\frac{\partial p^{\prime}(x, t)}{\partial t}+c\left(p^{\prime}\right) \frac{\partial p^{\prime}(x, t)}{\partial x}=0 \\
p^{\prime}(0, t)=\mathcal{P}_{a} \sin \omega_{a} t+\mathcal{P}_{b} \sin \omega_{b} t
\end{array}\right.
$$

Se exige a $\omega_{a}$ y $\omega_{b}$ ser múltiplos enteros positivos de un cierta frecuencia $\omega_{o}$, esto es:

$$
\omega_{a}=a \omega_{o}, \quad \omega_{b}=b \omega_{o}
$$

donde $a$ y $b$ son dos números naturales. Por otro lado, para cualesquiera $\mathcal{P}_{a}$ y $\mathcal{P}_{b}$ siempre se podrá elegir un valor $\mathcal{P}_{o}$ tal que:

$$
\mathcal{P}_{a}=A \mathcal{P}_{o}, \quad \mathcal{P}_{b}=B \mathcal{P}_{o}
$$

donde $A$ y $B$ son múltiplos reales dados. Estas condiciones permiten escribir la solución de Riemann (1.77) con $f(t)=\mathcal{P}_{a} \sin \omega_{a} t+\mathcal{P}_{b} \sin \omega_{b} t$ en la forma: 


$$
\begin{aligned}
p^{\prime}(x, t) & =\mathcal{P}_{o} A \sin \left[a\left(\omega_{o} t-k_{o} x+\frac{x}{\tilde{x}_{s h}} \frac{p^{\prime}(x, t)}{\mathcal{P}_{o}}\right)\right] \\
& +\mathcal{P}_{o} B \sin \left[b\left(\omega_{o} t-k_{o} x+\frac{x}{\tilde{x}_{s h}} \frac{p^{\prime}(x, t)}{\mathcal{P}_{o}}\right)\right]
\end{aligned}
$$

donde $\tilde{x}_{s h}$, es la distancia de choque para el caso mono-frecuencia (1.80). En el caso bi-frecuencial, para obtener la distancia de choque, se usará de nuevo (1.78). Con $\mathcal{P}_{a}$ y $\mathcal{P}_{b}$ ambas positivas, se tiene:

$$
\begin{aligned}
\tilde{\tilde{x}}_{s h} & =\frac{\rho c_{o}^{3}}{\beta\left(\omega_{a} \mathcal{P}_{a}+\omega_{b} \mathcal{P}_{b}\right)} \\
& =\frac{\tilde{x}_{s h}}{a A+b B}
\end{aligned}
$$

Se utiliza una doble tilde para denotar el caso bi-frecuencial. Se observa que en este caso, la distancia de choque se ve reducida una cantidad $a A+b B$ respecto al caso mono-frecuencia.

Para $x \leq \tilde{\tilde{x}}_{s h}$ la solución de Riemman (1.89) admite un desarrollo en la forma [3]:

$$
\begin{aligned}
& p^{\prime}(x, t)=\sum_{n=1}^{\infty} B_{n}(x) \sin \left[n \omega_{o}\left(t-\frac{x}{c_{o}}\right)\right] \\
& B_{n}(x)=2 \mathcal{P}_{o} \frac{\tilde{x}_{s h}}{n x} \sum_{l=-\infty}^{\infty} \sum_{m=-\infty}^{\infty} J_{l}\left(A \frac{n x}{\tilde{x}_{s h}}\right) J_{m}\left(B \frac{n x}{\tilde{x}_{s h}}\right)
\end{aligned}
$$

donde los índices $l$ y $m$ en el sumatorio deben cumplir la condición $l a+m b=n$.

Para $\omega_{a}>\omega_{b}$, esto es, para $a>b$, el desarrollo (1.91) puede expresarse como la suma de cuatro términos conocida como solución de Fenlon [3]: 


$$
\begin{aligned}
p^{\prime}(x, t) & =\sum_{l=1}^{\infty} \mathcal{A}_{l}(x) \sin \left[l \omega_{a}\left(t-\frac{x}{c_{o}}\right)\right] \\
& +\sum_{m=1}^{\infty} \mathcal{B}_{m}(x) \sin \left[m \omega_{b}\left(t-\frac{x}{c_{o}}\right)\right] \\
& +\sum_{l=1}^{\infty} \sum_{m=1}^{\infty} \mathcal{C}_{l m}(x) \sin \left[\left(l \omega_{a}+m \omega_{b}\right)\left(t-\frac{x}{c_{o}}\right)\right] \\
& +\sum_{l=1}^{\infty} \sum_{m=1}^{\infty} \mathcal{D}_{l m}(x) \sin \left[\left(l \omega_{a}-m \omega_{b}\right)\left(t-\frac{x}{c_{o}}\right)\right]
\end{aligned}
$$

con

$$
\begin{aligned}
& \mathcal{A}_{l}(x)=2 \mathcal{P}_{o} \frac{\tilde{x}_{s h}}{l a x} J_{l}\left(A \frac{\text { lax }}{\tilde{x}_{s h}}\right) J_{o}\left(B \frac{\text { lax }}{\tilde{x}_{s h}}\right) \\
& \mathcal{B}_{m}(x)=2 \mathcal{P}_{o} \frac{\tilde{x}_{s h}}{m b x} J_{l}\left(A \frac{m b x}{\tilde{x}_{s h}}\right) J_{o}\left(B \frac{m b x}{\tilde{x}_{s h}}\right) \\
& \mathcal{C}_{l m}(x)=2 \mathcal{P}_{o} \frac{\tilde{x}_{s h}}{n_{l m}^{+} x} J_{l}\left(A \frac{n_{l m}^{+} x}{\tilde{x}_{s h}}\right) J_{m}\left(B \frac{n_{l m}^{+} x}{\tilde{x}_{s h}}\right) \\
& \mathcal{D}_{l m}(x)=(-1)^{m} 2 \mathcal{P}_{o} \frac{\tilde{x}_{s h}}{n_{l m}^{-} x} J_{l}\left(A \frac{n_{l m}^{-} x}{\tilde{x}_{s h}}\right) J_{m}\left(B \frac{n_{l m}^{-} x}{\tilde{x}_{s h}}\right)
\end{aligned}
$$

con $n_{l m}^{ \pm}=l a \pm m b$.

La solución de Fenlon, permite ver explícitamente cómo interactúan dos ondas de frecuencias primarias $\omega_{a}$ y $\omega_{b}$ en régimen de no linealidad. El primer y segundo término en (1.93), muestran la aparición de armónicos de frecuencias $\omega_{a} \mathrm{y} \omega_{b}$, respectivamente. El tercer término, también evidencia la aparición de armónicos que son una combinación lineal suma de las frecuencias primarias, estos armónicos son de mayor frecuencia que sus frecuencias componentes $\omega_{a}$ y $\omega_{b}$. El cuarto término, es el más relevante, pues pone de manifiesto la aparición de armónicos que son una combinación lineal diferencia de las frecuencias primarias $\mathrm{y}$, por tanto, con componentes de menor frecuencia que $\omega_{a}$ y $\omega_{b}$. En consecuencia, si las frecuencias primarias son ultrasónicas, estas generan armónicos de baja frecuencia y, por tanto, 
pueden llegar a ser audibles. Este es un efecto relevante en los campos acústicos ultrasónicos de alta intensidad.

La solución de Fenlon (1.93) junto con la solución lineal (curva de color rojo) se grafica en la Figura 1.10 con $t=0$ y $0 \leq x \leq \tilde{\tilde{x}}_{s h}$. Se tomaron los siguientes valores numéricos:

$$
\begin{gathered}
c_{o}=1481.44 \mathrm{~m} / \mathrm{s}, \quad \rho_{o}=999.6 \mathrm{~kg} / \mathrm{m}^{3}, \quad \beta=10 \\
f_{o}=0.1 \mathrm{MHz}, \quad \mathcal{P}_{o}=1 \mathrm{MPa}, \quad \omega_{a}=21 \omega_{o}, \quad \omega_{b}=20 \omega_{o}, \quad A=B=1
\end{gathered}
$$

para estos valores, según (1.80) y (1.90), resulta ser $\tilde{x}_{s h}=51.72 \mathrm{~cm} \mathrm{y} \tilde{\tilde{x}}_{s h}=1.26 \mathrm{~cm}$.

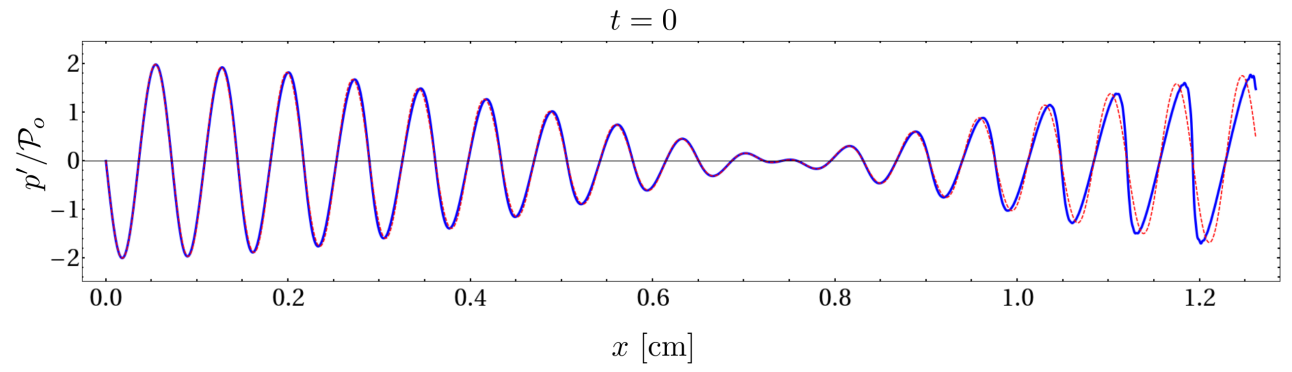

Figura 1.10: Deformación espacial de una onda armónica bi-frecuencial

\subsubsection{Excitación armónica mono-frecuencia en un medio viscoso}

Solución de Mendousse (1953). En este apartado, se considera el problema de contorno para la ecuación de Burgers viscosa (1.65) con una condición de frontera armónica mono-frecuencia:

$$
\left\{\begin{array}{l}
\frac{\partial p^{\prime}}{\partial x}=\frac{\beta p^{\prime}}{\rho_{o} c_{o}^{3}} \frac{\partial p^{\prime}}{\partial \tau}+\frac{\delta}{2 c_{o}^{3}} \frac{\partial^{2} p^{\prime}}{\partial \tau^{2}} \\
p^{\prime}(0, t)=\mathcal{P}_{o} \sin \omega_{o} t
\end{array}\right.
$$

En el caso viscoso, se mantiene la variable $\tau$ (tiempo de retardo) para hacer la notación mas compacta. Este problema admite una solución explícita conocida como solución de Mendousse [3] válida $\forall x$ : 


$$
p^{\prime}(x, t)=-\mathcal{P}_{o} \frac{4 \Gamma^{-1} \sum_{n=-\infty}^{\infty} n(-1)^{n} I_{n}\left(\frac{1}{2} \Gamma\right) e^{-n^{2} \alpha_{o} x} \sin n \omega_{o}\left(t-\frac{x}{c_{o}}\right)}{I_{o}\left(\frac{1}{2} \Gamma\right)+2 \sum_{n=-\infty}^{\infty}(-1)^{n} I_{n}\left(\frac{1}{2} \Gamma\right) e^{-n^{2} \alpha_{o} x} \cos n \omega_{o}\left(t-\frac{x}{c_{o}}\right)}
$$

donde $I_{n}$ es la función modificada de Bessel y $\Gamma$ es el llamado número de Gol'dberg definido como:

$$
\Gamma=\frac{L_{a}}{\tilde{x}_{s h}}=\frac{1}{\alpha_{o} \tilde{x}_{s h}}=\frac{2 \beta \mathcal{P}_{o}}{\delta \omega_{o} \rho_{o}}
$$

siendo $\tilde{x}_{s h}$ la distancia de choque para señales mono-frecuencia dada en (1.80), $\alpha_{o}$ el coeficientes de atenuación de pequeña señal definidos en (1.72) y $L_{a}$ la longitud de absorción dada en (1.73).

La solución de Mendousse (1.99) se grafica en la Figura 1.11 con distintos planos de observación. Para ello, se tomaron los siguientes valores numéricos:

$$
\begin{gathered}
c_{o}=1481.44 \mathrm{~m} / \mathrm{s}, \quad \rho_{o}=999.6 \mathrm{~kg} / \mathrm{m}^{3}, \quad \beta=10, \quad \delta=0.005 \mathrm{~m}^{2} / \mathrm{s} \\
f_{o}=0.1 \mathrm{MHz}, \quad \mathcal{P}_{o}=1 \mathrm{MPa}
\end{gathered}
$$

De estos valores se obtiene: $\tilde{x}_{s h}=51.72 \mathrm{~cm}, \alpha_{o}=0.30 \mathrm{~m}^{-1}, L_{a}=3.29 \mathrm{~m} \mathrm{y} \Gamma=6.37$.
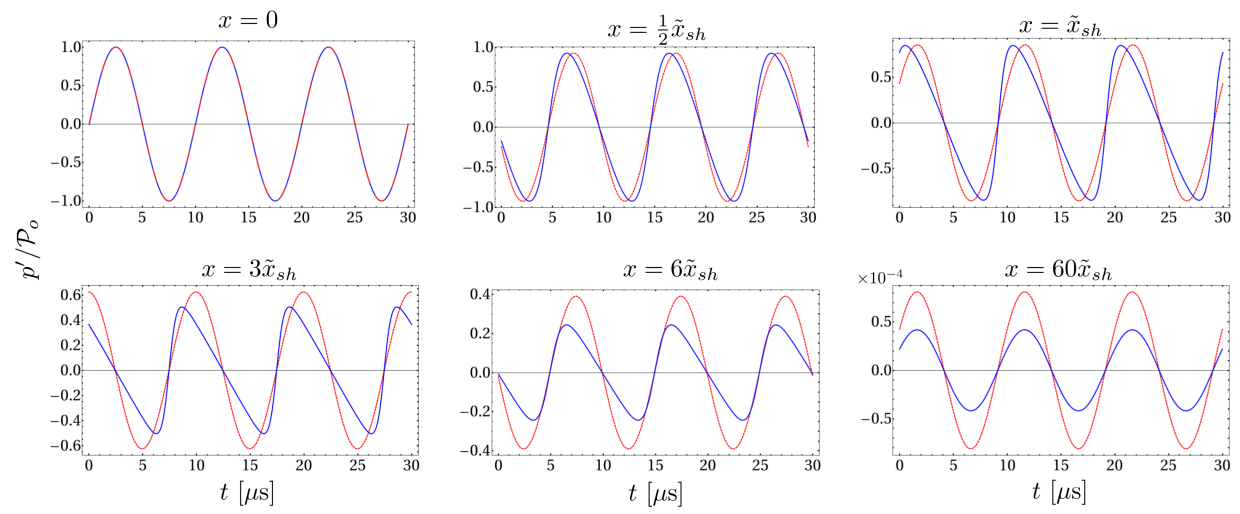

Figura 1.11: Solución de Mendousse en distintos planos de observación. La curva de color rojo muestra la solución lineal (1.74) 
En la Figura 1.11, se observa que en $x=0$ la solución de Mendousse reproduce el perfil de onda inicial dado por la condición de frontera en (1.98). En $x=0.5 \tilde{x}_{s h}$ la onda se deforma respecto a la solución lineal como era de esperar. En $x=\tilde{x}_{s h}$ se produce la onda de choque y la curva desarrolla pendientes verticales. Desde $x=\tilde{x}_{s h}$ a $x=3 \tilde{x}_{s h}$ la onda se transforma progresivamente hasta que su perfil adquiere la forma de un diente de sierra. Para $x>3 \tilde{x}_{s h}$ la onda evoluciona en forma de diente de sierra y se va atenuando debido a la disipación. Finalmente, para $x>>L_{a}$ la onda recupera el perfil de onda inicial pero muy reducido en amplitud.

\subsubsection{Excitación armónica bi-frecuencia en un medio viscoso}

Solución de Lardner (1982) [4]. En este apartado, se considera el problema de contorno para la ecuación de Burgers viscosa (1.65) con una condición de frontera armónica bi-frecuencia:

$$
\left\{\begin{array}{l}
\frac{\partial p^{\prime}}{\partial x}=\frac{\beta p^{\prime}}{\rho_{o} c_{o}^{3}} \frac{\partial p^{\prime}}{\partial \tau}+\frac{\delta}{2 c_{o}^{3}} \frac{\partial^{2} p^{\prime}}{\partial \tau^{2}} \\
p^{\prime}(0, t)=\mathcal{P}_{a} \sin \omega_{a} t+\mathcal{P}_{b} \sin \omega_{b} t
\end{array}\right.
$$

De forma análoga a como se estableció en el apartado 1.4.2, se supone que $\omega_{a}, \omega_{b}, \mathcal{P}_{a}$ y $\mathcal{P}_{b}$ pueden expresarse de la forma:

$$
\omega_{a}=a \omega_{o}, \quad \omega_{b}=b \omega_{o}, \quad \mathcal{P}_{a}=A \mathcal{P}_{o} \quad \mathcal{P}_{b}=B \mathcal{P}_{o}
$$

donde $a$ y $b$ son múltiplos naturales de una cierta frecuencia $\omega_{o}$ y $A$ y $B$ múltiplos reales de $\mathcal{P}_{o}$.

Este problema de contorno admite la siguiente solución conocida como solución de Lardner [5], la cual es la extensión bi-frecuencia de la solución de Mendousse (1.99):

$$
\begin{aligned}
& p^{\prime}(x, t)= \\
& -\mathcal{P}_{o} \frac{2 \Gamma^{-1} \sum_{l=-\infty}^{\infty} \sum_{m=-\infty}^{\infty} n_{l m}(-1)^{l+m} \mathcal{I}_{l m} e^{-n_{l m}^{2} \alpha_{o} x} \sin \left(l \omega_{a}+m \omega_{b}\right)\left(t-\frac{x}{c_{o}}\right)}{\sum_{l=-\infty}^{\infty} \sum_{m=-\infty}^{\infty}(-1)^{l+m} \mathcal{I}_{l m} e^{-n_{l m}^{2} \alpha_{o} x} \cos \left(l \omega_{a}+m \omega_{b}\right)\left(t-\frac{x}{c_{o}}\right)}
\end{aligned}
$$

donde $n_{l m}=l a+m b \mathrm{y}$ 


$$
\mathcal{I}_{l m}=I_{l}\left(\frac{A}{2 a} \Gamma\right) I_{m}\left(\frac{B}{2 b} \Gamma\right)
$$

con $\Gamma$ y $\alpha_{o}$ definidos en (1.100) y (1.72), respectivamente. El resultado (1.103) es solución del problema de contorno (1.101) con cualquier valor de $x$.

La Figura 1.12, muestra la solución de Lardner en el problema de contorno (1.101) con los siguientes valores numéricos:

$$
\begin{aligned}
& c_{o}=1481.44 \mathrm{~m} / \mathrm{s}, \quad \rho_{o}=999.6 \mathrm{~kg} / \mathrm{m}^{3}, \quad \beta=3.5, \quad \delta=0.0016 \mathrm{~m}^{2} / \mathrm{s} \\
& f_{o}=0.1 \mathrm{MHz}, \quad \mathcal{P}_{o}=2 \mathrm{MPa}, \quad \omega_{a}=9 \omega_{o}, \quad \omega_{b}=7 \omega_{o}, \quad A=B=1
\end{aligned}
$$

La curva de color rojo presenta la solución lineal:

$$
p^{\prime}(x, t)=\mathcal{P}_{o} e^{-\alpha_{a} t} \sin \omega_{a}\left(t-\frac{x}{c_{o}}\right)+\mathcal{P}_{o} e^{-\alpha_{b} t} \sin \omega_{b}\left(t-\frac{x}{c_{o}}\right)
$$

donde $\alpha_{a}$ y $\alpha_{b}$ son los coeficientes de atenuación en las frecuencias $\omega_{a}$ y $\omega_{b}$, respectivamente.

En la Figura 1.12, se observa que, durante la propagación se generan frecuencias que son combinaciones lineales de las frecuencias primarias $\omega_{a}=9 \omega_{o}$ y $\omega_{b}=7 \omega_{o}$. Es importante notar que para $x>1 \mathrm{~m}$ la frecuencia dominante es la frecuencia diferencia $(9-7 \quad 2)$ y los armónicos de mayor orden han sido fuertemente atenuados. Con $x=3 \mathrm{~m}$, la señal es básicamente una señal mono-frecuencia oscilando a la frecuencia diferencia $2 \omega_{o}$. La señal mantiene esta forma durante una gran distancia hasta que finalmente cede su energía al modo fundamental $\omega_{0}$. Esta evolución se muestra en la Figura 1.13

\subsubsection{Excitación modulada en un medio viscoso}

Como último ejemplo, se considera el problema de contorno para la ecuación de Burgers viscosa cuya condición de frontera es una función senoidal de amplitud $A_{p} \mathrm{y}$ frecuencia $\omega_{p}$ (frecuencia portadora) modulada en amplitud por una función $E(t)$ :

$$
\left\{\begin{array}{l}
\frac{\partial p^{\prime}}{\partial x}=\frac{\beta p^{\prime}}{\rho_{o} c_{o}^{3}} \frac{\partial p^{\prime}}{\partial \tau}+\frac{\delta}{2 c_{o}^{3}} \frac{\partial^{2} p^{\prime}}{\partial \tau^{2}} \\
p^{\prime}(0, t)=A_{p} E(t) \sin \omega_{p} t
\end{array}\right.
$$



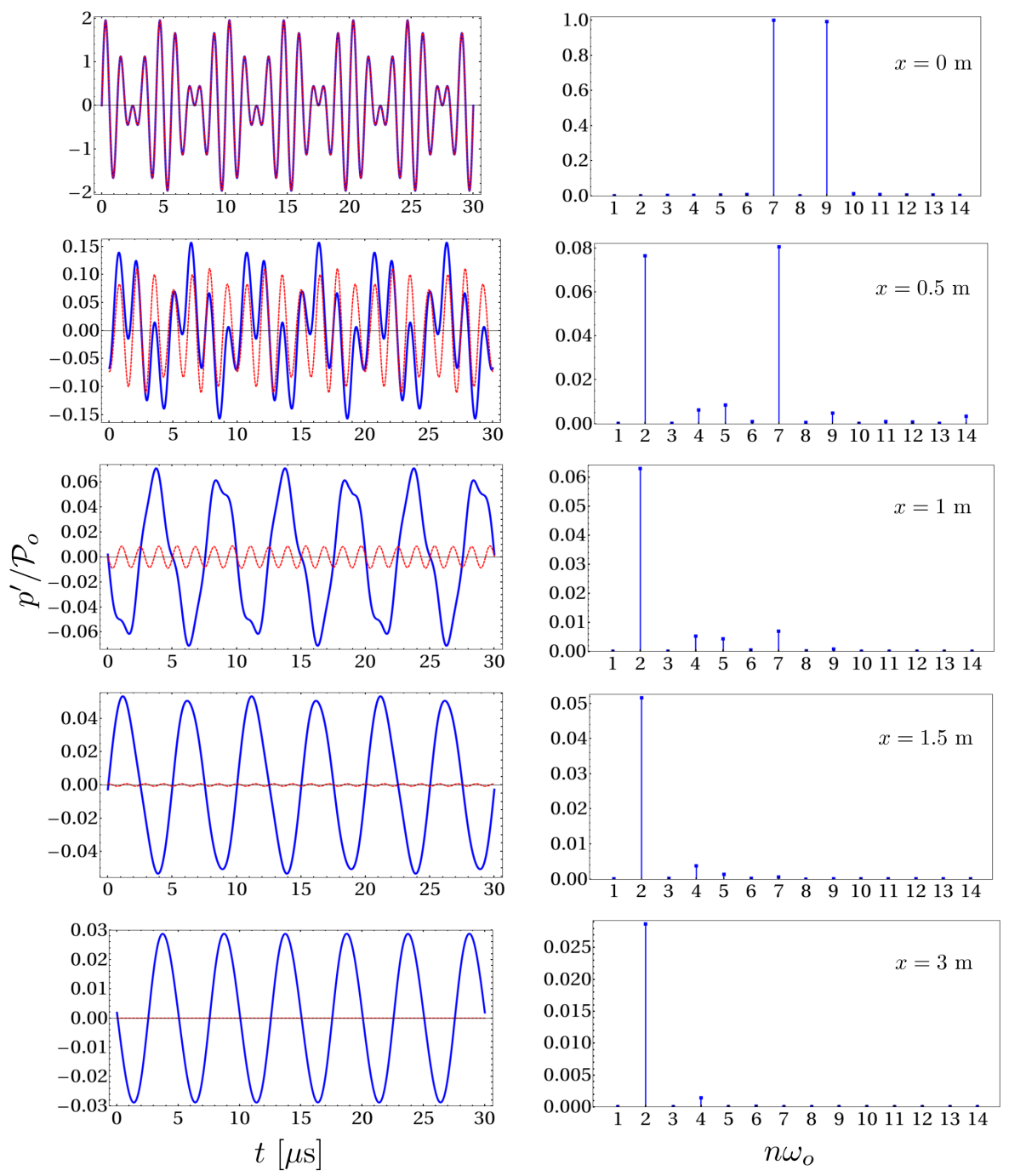

Figura 1.12: Solución de Lardner con $\omega_{a}=9 \omega_{o}$ y $\omega_{b}=7 \omega_{o}$ 

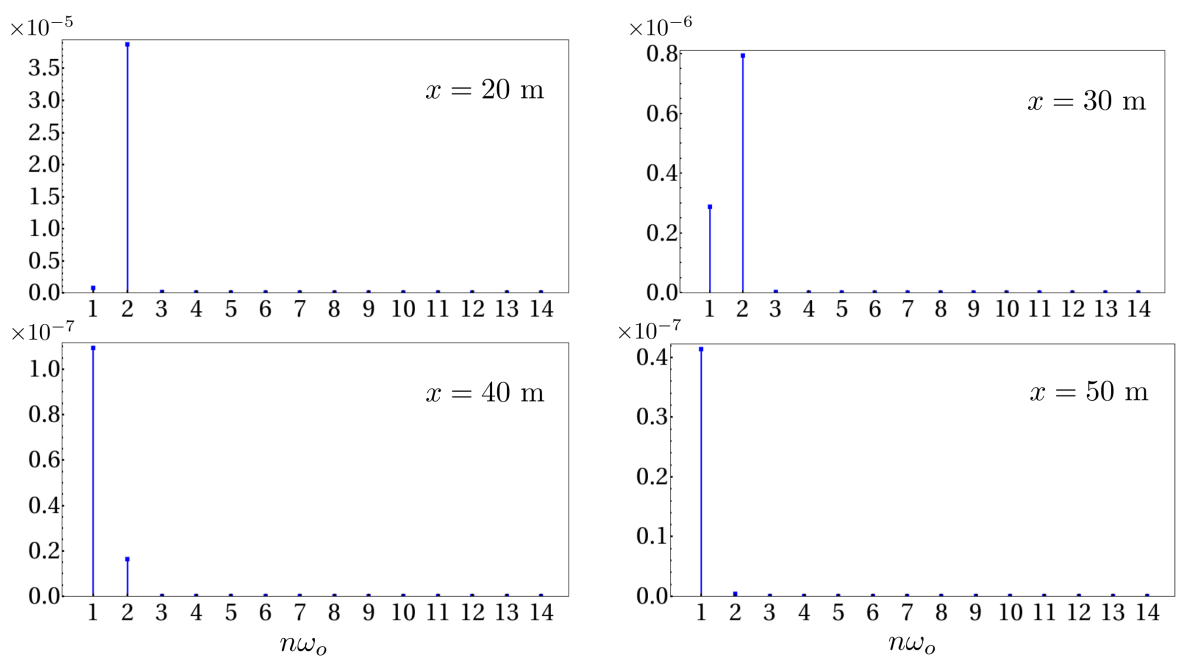

Figura 1.13: A grandes distancias de la frontera, toda la energía de la señal bi-frecuencia se transfiere al modo fundamental $\omega_{o}$

Con el objetivo de obtener una solución analítica, se tomará como función moduladora el caso más simple de todos los posibles, una función cosenoidal de frecuencia $\omega_{m}$ $\left(<\omega_{p}\right)$. Así pues, la condición de frontera en (1.106) toma la forma:

$$
\begin{aligned}
p^{\prime}(0, t) & =A_{p} \cos \left(\omega_{m} t\right) \sin \left(\omega_{p} t\right) \\
& =\frac{A_{p}}{2} \sin \left(\omega_{p}+\omega_{m}\right) t+\frac{A_{p}}{2} \sin \left(\omega_{p}-\omega_{m}\right) t \\
& =\mathcal{P}_{o} \sin \omega_{a} t+\mathcal{P}_{o} \sin \omega_{b} t
\end{aligned}
$$

con $\mathcal{P}_{o}=1 / 2 A_{p}, \omega_{a}=\omega_{p}+\omega_{m}$ y $\omega_{b}=\omega_{p}-\omega_{m}$. El problema queda reducido a una condición de contorno bi-frecuencia como la tratada en el apartado anterior. Para poder usar la solución de Lardner (1.103) se exige a $\omega_{a}$ y $\omega_{b}$ ser múltiplos naturales de una cierta frecuencia $\omega_{o}$, en consecuencia, se tiene lo siguiente:

$$
\begin{aligned}
& \omega_{p}+\omega_{m}=a \omega_{o} \\
& \omega_{p}-\omega_{m}=b \omega_{o}
\end{aligned}
$$

Despejando $\omega_{p}$ y $\omega_{m}$ del sistema de ecuaciones (1.108), se tiene: 


$$
\begin{aligned}
& \omega_{p}=\frac{a+b}{2} \omega_{o} \\
& \omega_{m}=\frac{a-b}{2} \omega_{o}
\end{aligned}
$$

El resultado (1.110) muestra que, la frecuencia diferencia es igual al doble de la frecuencia moduladaora. Esto es:

$$
\omega_{a}-\omega_{b}=(a-b) \omega_{o}=2 \omega_{m}
$$

Como ejemplo numérico se considera la señal modulada:

$$
\begin{gathered}
p^{\prime}(0, t)=A_{p} \cos \left(\omega_{m} t\right) \sin \left(\omega_{p} t\right) \\
A_{p}=4 \mathrm{MPa}, \quad f_{m}=50 \mathrm{kHz}, \quad f_{p}=650 \mathrm{kHz}
\end{gathered}
$$

De (1.109) y (1.110), es fácil comprobar que con estos valores $\omega_{m}$ y $\omega_{p}$ el problema se reduce a una excitación bi-frecuencia con:

$$
\begin{gathered}
p^{\prime}(0, t)=\mathcal{P}_{o} \sin \left(\omega_{a} t\right)+\mathcal{P}_{o} \sin \left(\omega_{b} t\right) \\
\mathcal{P}_{o}=2 \mathrm{MPa}, \quad f_{o}=100 \mathrm{kHz} \quad \omega_{a}=7 \omega_{o}, \quad \omega_{b}=6 \omega_{o}
\end{gathered}
$$

La Figura 1.14, presenta la evolución de la señal modulada en un medio definido por los siguientes valores:

$$
c_{o}=1481.44 \mathrm{~m} / \mathrm{s}, \quad \rho_{o}=999.6 \mathrm{~kg} / \mathrm{m}^{3}, \quad \beta=3.5, \quad \delta=0.0016 \mathrm{~m}^{2} / \mathrm{s}
$$



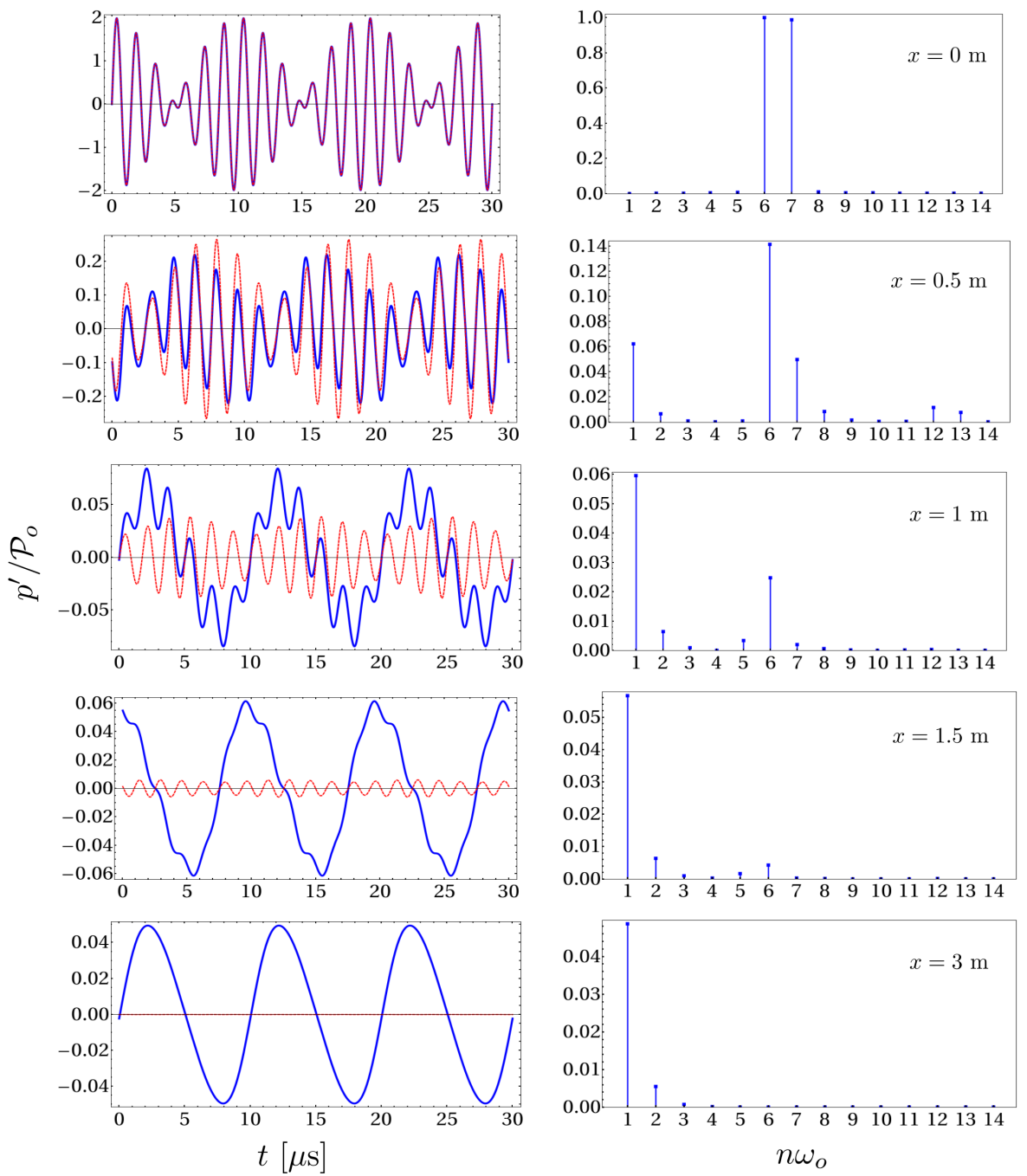

Figura 1.14: Solución de Lardner para una señal modulada con $\omega_{m}=50 \mathrm{kHz}$ y $\omega_{p}=650$ $\mathrm{kHz}$. La curva de color rojo muestra la solución lineal

De la Figura 1.14 se puede observar que, con $x>3 \mathrm{~m}$ la frecuencia dominante es la frecuencia diferencia $\omega_{a}-\omega_{b}=(2-1) \omega_{o}=\omega_{o}$. Y como era de esperar, según (1.111), la frecuencia diferencia es el doble de la frecuencia moduladora $2 \omega_{m}=\omega_{o}$ $(2 \cdot 50 \mathrm{kHz}=100 \mathrm{kHz})$. 


\subsection{Efecto paramétrico}

La solución del problema de contorno (1.101) muestra que, cuando dos ondas armónicas lineales de frecuencias $\omega_{a}$ y $\omega_{b}$ (llamadas primarias) se propagan en la misma dirección, existe una interacción mutua entre ellas debido a la no linealidad, formándose ondas secundarias de frecuencias que son combinaciones lineales suma y diferencia de las frecuencias primarias. Este resultado también puede observarse explícitamente de la solución de Fenlon (1.93). Por otra parte, los efectos disipativos del medio, atenúan las ondas de mayor frecuencia haciéndolas desaparecer, para longitudes de propagación mucho mayores que la longitud de absorción. Como consecuencia, la onda diferencia $\omega_{d} \equiv \omega_{a}-\omega_{b}$ es la que se propaga a mayor distancia. Este efecto se conoce con el nombre de efecto paramétrico y la señal diferencia $\omega_{d}$ se denomina señal paramétrica. Este resultado es la base teórica del trabajo experimental presentado en esta tesis.

A lo largo de la longitud de interacción, la frecuencia diferencia se comporta como un conjunto de fuentes virtuales puntuales, resultado de la interacción no lineal de las altas frecuencias emitidas por el transductor o conjunto de transductores (array paramétrico). Cuanto mayor sea la longitud de interacción, mayor será la presión que se pueda lograr al momento de la emisión de las señales. Por lo tanto, la absorción determina la longitud de interacción. La longitud de absorción $L_{A}$ se puede calcular como:

$$
L_{A}=\frac{1}{\alpha_{p}}
$$

donde $\alpha_{p}$ es el coeficiente de absorción dada en $N_{p} / m$, para la onda primaria.

Esta distancia puede estar en el campo cercano o lejano de la interacción de las frecuencias primarias, conocida comúnmente como distancia de Rayleigh, que es la distancia a partir de la cual se puede considerar que la onda pasa de comportarse como una onda plana a una onda esférica. A partir de esta distancia la onda se atenúa $6 \mathrm{~dB}$ al duplicar la distancia a la fuente [6]. Dado que en esta tesis se estudia un fenómeno de emisión, debe tenerse en cuenta el campo cercano del transductor para las frecuencias primarias radiadas, donde la radiación está contenida principalmente en un cilindro de longitud la distancia de Rayleigh y de sección correspondiente a la superficie del transductor mientras que, en el campo lejano, empieza la divergencia esférica de la radiación y la energía se expande más rápidamente.

la distancia de Rayleigh según la teoría de Westervelt [7], se expresa como:

$$
L_{R}=\frac{1}{2} k_{p} a^{2}=\frac{\omega_{p} a^{2}}{2 c_{o}}
$$


Donde $a$ es el radio del transductor emisor, $k_{p}$ y $\omega_{p}$ es el número de onda y la frecuencia de la señal primaria, y $c_{o}$ la velocidad del sonido.

Ahora bien, cuando las señales primarias son dos tonos puros de frecuencias $\omega_{a} \mathrm{y} \omega_{b}$, la señal paramétrica es una onda de una sola componente. En este caso, el efecto paramétrico se denomina, efecto paramétrico dual. La Figura 1.15 muestra cómo evoluciona la señal dual a lo largo de la distancia de propagación para dos tonos de frecuencias $\omega_{a} / 2 \pi=2.3 \mathrm{MHz}$ y $\omega_{b} / 2 \pi=2 \mathrm{MHz}$. Las altas frecuencias se absorben rápidamente en el medio y, para $x>20 \mathrm{~cm}$, la señal es básicamente mono-frecuencia oscilando a la frecuencia paramétrica $0.3 \mathrm{MHz}$. Para obtener esta gráfica, se utilizó la solución de Lardner (1.103) con $\mathcal{P}_{a}=\mathcal{P}_{b}=1 \mathrm{MPa}$ y los siguientes parámetros para el medio:

$$
c_{o}=1481.44 \mathrm{~m} / \mathrm{s}, \quad \rho_{o}=999.6 \mathrm{~kg} / \mathrm{m}^{3}, \quad \beta=10, \quad \delta=0.0016 \mathrm{~m}^{2} / \mathrm{s}
$$

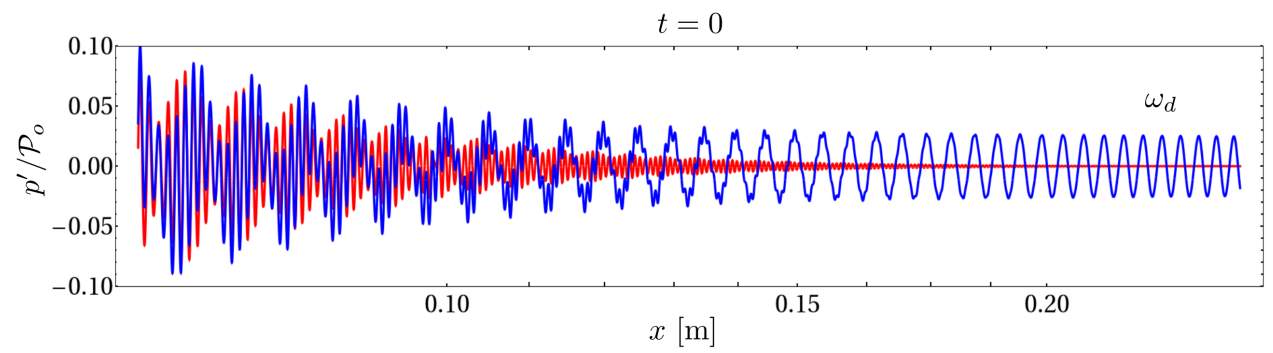

Figura 1.15: Efecto paramétrico dual a lo largo de la distancia de propagación (el eje $x$ se tomó en escala logarítmica desde $x=7 \mathrm{~cm}$ a $x=25 \mathrm{~cm}$ ). La curva de color rojo muestra la solución lineal

Una vez obtenida la solución a la ecuación de onda (1.53) para la frecuencia diferencia $\omega_{d}$, que se compone de contribuciones de las onda primarias $\omega_{a}$ y $\omega_{b}$ y después de diferentes procesos matemáticos desarrollados por Westervelt en $[1,7]$, se presenta la amplitud de $\omega_{d}=2 \pi f_{d}$ en campo lejano del transductor expresada a través de la siguiente ecuación:

$$
p_{d}(r, \theta)=-\frac{\omega_{d}^{2} p_{1} p_{2} \beta S_{o}}{8 \pi \rho_{o} c_{o}^{4} r \alpha_{p}}\left[\frac{1}{1+\frac{k_{d}^{2}}{\alpha_{p}^{2}} \sin ^{4}(\theta / 2)}\right]
$$

Donde $p_{d}$ es la presión de la frecuencia diferencia producida por la interacción no lineal de las frecuencias primarias $\left(\omega_{a} \mathrm{y} \omega_{b}\right), \omega_{d}$ es la frecuencia diferencia, $p_{1}$ y $p_{2}$, 
corresponden la amplitud de la presión de las señales primarias $\left(\omega_{1}\right.$ y $\left.\omega_{b}\right), \beta$ es el coeficiente de no linealidad en el medio, $S_{o}$, es la sección donde se intersectan los haces primarios y se aproxima a $\pi\left(2 \theta_{m} / 2\right)^{2}, \rho_{o}$ y $c_{o}$, son la densidad y velocidad del sonido en el medio, $r$ es la distancia de propagación de la onda acústica, $\alpha_{p}$ y $k_{d}$ es el coeficiente de abosrción de las ondas primarias y el número de onda de la frecuencia diferencia, y $\theta$ es es ángulo.

El campo acústico de la frecuencia diferencia corresponde a dos términos, el primero es la propagación a lo largo del eje de radiación, y el segundo, a la directividad del paramétrico. Las características que se pueden extraer de la expresión de Westervelt (1.114) son [8]:

El campo acústico de la frecuencia diferencia corresponde a dos términos, el primero es la presión a través del eje y a una distancia a $1 \mathrm{~m}$ del transductor se obtiene el nivel equivalente para la fuente paramétrica (frecuencia diferencia). El segundo término de la derecha es la función de directividad. Las características que se pueden extraer de la expresión de Westervelt (1.114) son [8,9]:

- La presión de la frecuencia secundaria aumenta con el cuadrado de las presiones primarias, de modo que el nivel de presión es mayor para elevadas frecuencias del paramétrico, aunque estos valores dependen primordialmente de las propiedades del transductor así como de la distancia a la que se forma el campo lejano.

- La atenuación del paramétrico disminuye inversamente con la distancia $1 / r$, de modo que al tratar con niveles de presión ésta disminuye $6 \mathrm{~dB}$ al duplicar la distancia.

- Respecto al segundo término (directividad), esta función muestra un lóbulo de directividad estrecho alrededor de $\theta=0$, y una ausencia completa de lóbulos laterales. Esta es una propiedad notable de los arrays paramétricos.

Un ejemplo teórico de esta directividad y amplitud se podrá observar en el Capítulo 4, Figura 4.4 y Figura 4.5 respectivamente, donde se tiene que el patrón de directividad para la frecuencia paramétrica es muy parecido a la señal primaria. En general, la directividad del paramétrico, es muy estrecha y con disminución o ausencia de lóbulos laterales.

Posterior a los estudios de Westervelt (1.114), que supuso que las ondas primarias eran cercanas entre sí permitiendo que la distribución de las fuentes pudiera representarse en forma de linea (fuentes puntuales) ubicadas a o largo del eje de las ondas primarias y no mas allá de la distancia de Rayleigh, dado que fuera de ella las ondas primarias no se pueden considerar ondas planas cercanas debido a los efectos de la dispersión esférica. Berktay en 1965 [10,11], desarrolló estudios teóricos y medidas experimentales 
mas complejos teniendo en cuenta la interacción de las frecuencias primarias a ondas cilíndricas y esféricas, y en vez de estudiar dos frecuencias primarias como Westervelt, basó sus estudios en la generación de señales paramétricas mediante el uso de una señal primaria (portadora) de banda ancha, en cuyo caso, un número infinito de frecuencias interactúan generando una señal paramétrica de banda ancha y el efecto paramétrico se denomina efecto paramétrico de banda ancha. Este sería para una condición de contorno genérica como la tratado en el problema (1.106):

$$
p^{\prime}(0, t)=A_{p} E(t) \sin \omega_{p} t
$$

donde $\omega_{p}$ es la frecuencia portadora y la amplitud modulada $E(t)$ puede ser cualquier función que varía lentamente en comparación con $\sin \omega_{p} t$. Estos problemas serán tratados en el siguiente apartado.

\subsection{Ecuación KZK y solución de Berktay}

Hasta ahora, sólo se han considerado soluciones a la ecuación de Burgers con distintas condiciones de contorno, cuyo objetivo final era definir de forma simple pero formal el efecto paramétrico. Ahora bien, esta ecuación sólo es válida, para estudiar el comportamiento de ondas planas que se propagan estrictamente en una dirección. Cuando las ondas son generadas por fuentes de dimensiones finitas (como un transductor), las ondas no pueden ser tratadas como planas y la ecuación de Burgers deja de ser útil. Una extensión de la ecuación de Burgers que toma en cuenta los efectos de la difracción (debido al tamaño finito de la fuente que excita el medio) puede ser obtenida a partir de la ecuación de Westervelt, tal y como se hizo con la ecuación de Burgers. Esta ecuación es conocida como KZK, llamada así por sus autores Khokhlov, Zabolotskaya y Kuznetsov [12,13]. Esta ecuación, fue originalmente obtenida para describir la propagación no lineal de haces acústicos. Cuando se habla de haz, se entiende un campo acústico que varía más lentamente en la dirección transversal que en la dirección longitudinal o de propagación. En otras palabras, en un haz acústico la energía de la onda está localizada en un entorno alrededor de la dirección de propagación y se supone que el haz es razonablemente direccional o poco divergente. A groso modo, se puede visualizar un haz como un cono angosto en cuyo interior se encuentra la mayor parte de la energía de la perturbación. La propiedad de direccionalidad queda asegurada si $\frac{2 \pi a}{\lambda}>>1$, donde $a$ es el radio característico de la fuente [3].

Si se toma el eje $+z$ como dirección de propagación (lo que es habitual), la ecuación KZK se puede obtener haciendo el siguiente cambio de variables [14]: 


$$
x_{1}=\sqrt{\epsilon} x, \quad y_{1}=\sqrt{\epsilon} y, \quad z_{1}=\epsilon z, \quad \tau=t-\frac{z}{c_{o}}
$$

donde $\epsilon$ se tratará como un término de primer orden. Con este cambio de variables el operador laplaciano toma la forma:

$$
\nabla^{2}=\epsilon\left(\frac{\partial^{2}}{\partial x_{1}^{2}}+\frac{\partial^{2}}{\partial y_{1}^{2}}\right)+\epsilon^{2} \frac{\partial^{2}}{\partial z_{1}^{2}}-\epsilon \frac{2}{c_{o}} \frac{\partial^{2}}{\partial z_{1} \partial \tau}+\frac{1}{c_{o}^{2}} \frac{\partial^{2}}{\partial \tau^{2}}
$$

Sustituyendo (1.117) en la ecuación de Westervelt (1.64) y despreciando los términos de orden superior a dos, se obiene:

$$
\epsilon\left(\frac{\partial^{2} p^{\prime}}{\partial x_{1}^{2}}+\frac{\partial^{2} p^{\prime}}{\partial y_{1}^{2}}\right)-\epsilon \frac{2}{c_{o}} \frac{\partial^{2} p^{\prime}}{\partial z_{1} \partial \tau}=-\frac{\beta}{\rho_{o} c_{o}^{4}} \frac{\partial^{2} p^{\prime 2}}{\partial \tau^{2}}-\frac{\delta}{c_{o}^{4}} \frac{\partial^{3} p^{\prime}}{\partial \tau^{3}}
$$

Deshaciendo el cambio de variables en las coordenadas espaciales, se tiene la ecuación KZK en función del tiempo de retardo $\tau$ :

$$
\frac{\partial^{2} p^{\prime}}{\partial z \partial \tau}=\frac{c_{o}}{2} \nabla_{\rho}^{2} p^{\prime}+\frac{\delta}{2 c_{o}^{3}} \frac{\partial^{3} p^{\prime}}{\partial \tau^{3}}+\frac{\beta}{2 \rho_{o} c_{o}^{3}} \frac{\partial^{2} p^{\prime 2}}{\partial \tau^{2}}
$$

con $p^{\prime}=p^{\prime}(\vec{\rho}, z, \tau)$, donde $\vec{\rho}=x \hat{i}+y \hat{j}$ denota las coordenadas transversales $(x, y)$ y $\nabla_{\rho}^{2}=\frac{\partial^{2}}{\partial x^{2}}+\frac{\partial^{2}}{\partial y^{2}}$ es el laplaciano en dicha dirección.

Se puede interpretar la ecuación KZK como la aproximación paraxial de la ecuación de Westervelt. Dicha ecuación, contiene tres términos a la derecha de la igualdad en (1.119): el primer término tiene en cuenta la difracción parabólica, el segundo los efectos disipativos y el tercero la no linealidad.

Hasta ahora, no se dispone de una solución analítica para la ecuación de onda parabólica KZK, por ello, es necesario recurrir a su solución numérica [15], la cual es ampliamente usada. Sin embargo, como alternativa a la solución numérica, una aproximación analítica cuasilineal se puede obtener usando el método de perturbaciones. Para ello, se supone que la presión acústica se puede linealizar de la forma:

$$
p^{\prime}(\vec{\rho}, z, \tau)=p_{1}(\vec{\rho}, z, \tau)+p_{2}(\vec{\rho}, z, \tau)+\cdots
$$


donde $p_{1}$ es la solución lineal (primera aproximación) y $p_{2}$ (segunda aproximación) es una pequeña corrección a $p_{1}$ basada en la suposición $\left|p_{2}\right|<<\left|p_{1}\right|$. Los términos $p_{1}$ y $p_{2}$ se suelen nombrar como haz primario y secundario, respectivamente.

Sustituyendo (1.120) en (1.119) y despreciando los términos de orden superior a dos, se obtiene el siguiente sistema de ecuaciones:

$$
\left\{\begin{array}{l}
\frac{\partial^{2} p_{1}}{\partial z \partial \tau}-\frac{c_{o}}{2} \nabla_{\rho}^{2} p_{1}-\frac{\delta}{2 c_{o}^{3}} \frac{\partial^{3} p_{1}}{\partial \tau^{3}}=0 \\
\frac{\partial^{2} p_{2}}{\partial z \partial \tau}-\frac{c_{o}}{2} \nabla_{\rho}^{2} p_{2}-\frac{\delta}{2 c_{o}^{3}} \frac{\partial^{3} p_{2}}{\partial \tau^{3}}=\frac{\beta}{2 \rho_{o} c_{o}^{3}} \frac{\partial^{2} p_{1}^{2}}{\partial \tau^{2}}
\end{array}\right.
$$

Así, el problema no lineal (1.119) queda reducido a un sistema de dos ecuaciones diferenciales lineales. La ecuación homogénea (1.121a) permite determinar $p_{1}$. Una vez que $p_{1}$ se determina, la ecuación no homogénea $(1.121 \mathrm{~b})$ permite obtener $p_{2}$.

Para encontrar una solución al sistema de ecuaciones (1.121a)-(1.121b) es necesario fijar una condición de frontera. Se supone que la condición de frontera está definida por un transductor circular de radio $a$ excitado con una señal $f(t)$ y cuya superficie se hace coincidir con el plano $z=0$. La Figura 1.16 muestra la geometría del problema.

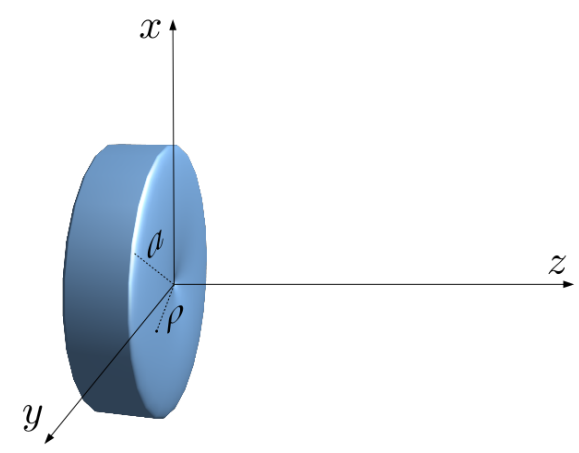

Figura 1.16: Transductor circular de radio a cuya superficie coincide con el plano $z=0$

Matemáticamente, la condición de frontera se expresa como:

$$
p^{\prime}(\vec{\rho}, 0, t)=\left\{\begin{array}{lll}
f(t) & \text { si } & \rho \leq a \\
0 & \text { si } & \rho>a
\end{array}\right.
$$


El objetivo de esta tesis es la generación de señales paramétricas. Como se comentó en el apartado anterior, existen dos métodos; el primero, es tomar una señal dual de frecuencias $\omega_{a}$ y $\omega_{b}$, en este caso $f(t)=\mathcal{P}_{a} \sin \omega_{a} t+\mathcal{P}_{b} \sin \omega_{b} t$. El sistema de ecuaciones (1.121a)-(1.121b) se puede integrar usando el método de la función de Green. Un desarrollo completo de la solución paramétrica dual donde también se hace un estudio de directividad del haz se encuentra en [3].

En esta tesis, el interés se centra en el efecto paramétrico de banda ancha, en cuyo caso $f(t)=A_{p} E(t) \sin \omega_{p} t$. Este problema fue tratado por Berktay en 1965 [10], el cual propuso una solución a la ecuación de Westervelt llamada auto-demodulación, empleando señales banda ancha, esto quiere decir, que se aplica una modulación AM [16]. Esta modulación, consiste en emitir una onda primaria modulada en amplitud mediante una baja frecuencia, cuya demodulación se da en el medio a través de los efectos no lineales que se generan. La solución a dicho problema se puede obtener como sigue.

El primer haz es aproximado por una onda localmente plana perfectamente colimada. Esto es,

$$
p_{1}(\vec{\rho}, z, \tau) \simeq\left\{\begin{array}{lll}
A_{p} e^{-\alpha z} E(\tau) \sin \omega_{p} \tau & \text { si } & \rho \leq a \\
0 & \text { si } & \rho>a
\end{array}\right.
$$

donde $\alpha$ es el coeficiente de absorción en la frecuencia $\omega_{p}$ y $E(t)$ es cualquier función que varía lentamente en comparación con $\sin \omega_{p} t$. Se supone $\alpha$ suficientemente grande, así, la interacción no lineal está confinada en el campo cercano del haz.

El haz secundario $p_{2}$ se determina por $p_{1}^{2}$ según (1.121b), el cual contiene el término $E^{2}(\tau) \sin ^{2} \omega_{p} t=1 / 2 E(\tau)\left[1-\cos 2 \omega_{p} t\right]$. Este término consiste de altas frecuencias debido a la parte $E^{2}(\tau) \cos 2 \omega_{p} t$ y bajas frecuencia asociado con $E^{2}(\tau)$. Las altas frecuencia se atenúan más rápidamente que la bajas, así, se considera tan sólo la contribución de bajas frecuencia en el campo lejano. En consecuencia, se puede tomar la siguiente aproximación para $p_{1}^{2}$ :

$$
p_{1}^{2}(\vec{\rho}, z, \tau) \simeq\left\{\begin{array}{lll}
\frac{1}{2} A_{p}^{2} e^{-2 \alpha z} E^{2}(\tau) & \text { si } & \rho \leq a \\
0 & \text { si } & \rho>a
\end{array}\right.
$$

Una vez se tiene $p_{1}^{2}$, se obtiene $p_{2}$ a partir de (1.121b). Si se ignora los efectos de la absorción haciendo $\delta=0$, la solución axial para $p_{2}$ viene dada por la integral de volumen [3]: 


$$
p_{2}(0, z, \tau)=\frac{\beta}{4 \pi \rho_{o} c_{o}^{4}} \frac{\partial^{2}}{\partial \tau^{2}} \int_{0}^{z} \int_{0}^{2 \pi} \int_{0}^{\infty} p_{1}^{2}\left(\rho^{\prime}, z^{\prime}, \tau-\frac{\rho^{\prime 2}}{2 c_{o}\left(z-z^{\prime}\right)}\right) \frac{\rho^{\prime} d \rho^{\prime} d z^{\prime}}{z-z^{\prime}}
$$

Si ahora se aplica la aproximación de campo lejano $\left(z>>L_{R}\right)$, el término de fase $\rho^{\prime 2} / 2 c_{o}\left(z-z^{\prime}\right)$ se puede ignorar, el término $d z^{\prime} /\left(z-z^{\prime}\right)$ puede ser reemplazado por $d z^{\prime} / z$ y el límite superior de la integral en $z^{\prime}$ se extiende a $\infty$. Bajo esta aproximación de campo lejano, la integral (1.125) se reduce a:

$$
p_{2}(0, z, \tau)=\frac{\beta}{2 \rho_{o} c_{o}^{4} z} \frac{\partial^{2}}{\partial \tau^{2}} \int_{0}^{\infty} \int_{0}^{\infty} p_{1}^{2}\left(\rho^{\prime}, z^{\prime}, \tau\right) \rho^{\prime} d \rho^{\prime} d z^{\prime}
$$

Sustituyendo (1.124) en (1.126), se obtiene la distribución de presiones de la frecuencia paramétrica a lo largo del eje de radiación, cumpliendo con la condición de campo lejano de Berktay, donde $\tau=t-x / c_{o}$ :

$$
p_{2}(0, z, \tau)=\frac{\beta A_{p}^{2} a^{2}}{16 \rho_{o} c_{o}^{4} \alpha z} \frac{\partial^{2} E^{2}(\tau)}{\partial \tau^{2}}
$$

De esta solución, se tiene que las propiedades del campo acústico paramétrico son:

- El resultado presente tiene un comportamiento no lineal, debido a que la onda resultante será proporcional a la segunda derivada de la envolvente $E(t)$ al cuadrado de la señal emitida.

- La amplitud de la onda paramétrica depende del cuadrado de la amplitud de la onda emitida.

En general, de la solución de Westervelt y la de Berktay, se tiene que, el haz secundario presenta un comportamiento directivo idéntico al de las frecuencias primarias. Los estudios teóricos y experimentales demostraron que en el campo lejano la $\omega_{d}=\omega_{a}-\omega_{b}$, tendría haces muy estrechos con lóbulos laterales muy bajos en comparación con los haces acústicos irradiados convencionalmente, característica muy importante cuando esto se desea aplicar a las comunicaciones acústicas submarinas [17] en aguas poco profundas ya que su alta directividad podrá combatir la propagación multitrayecto. La principal desventaja de la transducción paramétrica es la pérdida de potencia equivalente aproximadamente a $20 \mathrm{~dB}$ [18]. Sin embargo, una ventaja para la propagación a largas distancias es que la $f_{d}$ tiene una absorción relativamente baja.

Como ejemplo numérico para determinar la distancia de absorción y de Rayleigh se cosideran los siguientes valores que corresponden a los empleados en los experimentos 
de esta tesis, con el fin de aplicar las aproximaciones que simplifican el modelado de transmisión. Dependiendo de las distancias descritas anteriormente, se deben usar diferentes modelos matemáticos para predecir correctamente los niveles de fuente de la onda secundaria.

Con todo lo anterior, se determina la distancia entre transductores en un espacio controlado (tanque de agua), para tener en cuenta que las bajas frecuencias paramétricas se generen por interacción no lineal del mismo medio, con ello, se deberá medir en campo lejano.

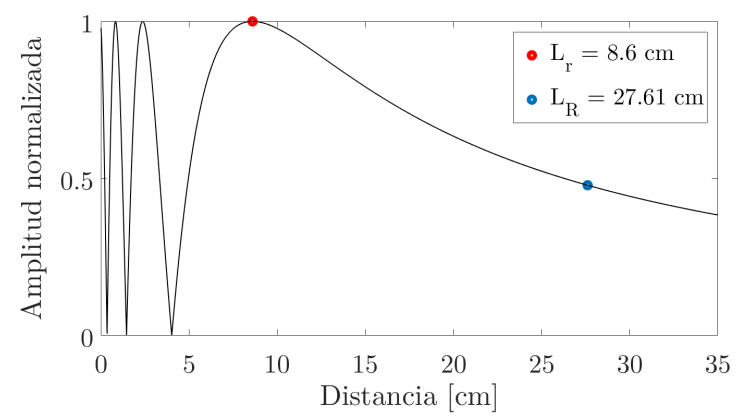

Figura 1.17: Distancia del último máximo del patrón de interferencias $L_{r}$ que se encuentra a $10 \mathrm{~cm}$ entre transductores, y la distancia de Rayleigh $L_{R}$ a $33 \mathrm{~cm}$ de una señal propagada en piscina

Las distancias presentadas en la Figura 1.17, muestran que, para medir en campo lejano o distancia de Rayleigh, la distancia entre transductores debe ser igual o superior a $33 \mathrm{~cm}$.

Dependiendo de la relación entre estas distancias, se trabajará en un rango u otro que presentará, sus propias características en función de los haces primarios y las distintas condiciones del medio de interacción, cuya representación, permitirá conocer las aproximaciones adecuadas en la teoría y contrastarlas con las resultados experimentales. La Figura 1.18, muestra las diferentes combinaciones [19].

De entre las diferentes combinaciones de la Figura 1.18 para el rango de trabajo de una fuente paramétrica: Westervelt [7], Moffett y Mellen [20] y Berktay y Leahy [11], el que más se ajusta a los resultados experimentales cuando se mide en piscina, es el modelo de Berktay ya que cumple la condición que $L_{s}>L_{A}>L_{R}$, tal como se observa en la Tabla 1.1.

La frecuencia más alta que se emplea en el transductor proyector Airmar P19 es 200 $\mathrm{kHz}$. 


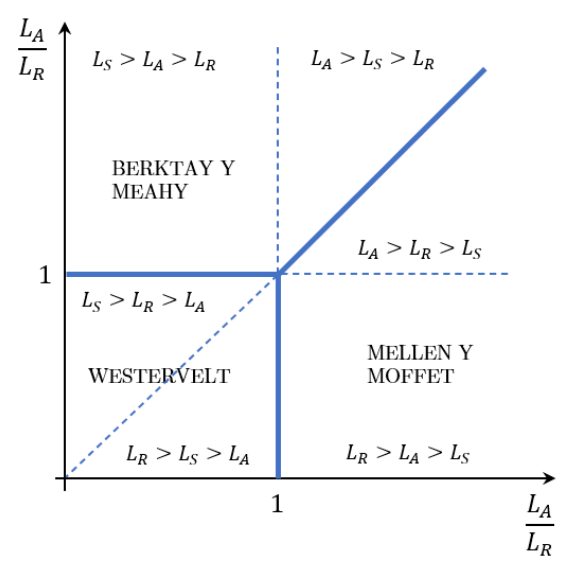

Figura 1.18: Combinaciones para los diferentes rangos de trabajo para el paramétrico donde los límites entre las regiones de validez de los modelos se encuentran resaltados en azul

\begin{tabular}{lcccccccc}
\hline EMISOR & $\begin{array}{c}\boldsymbol{f}_{\text {max }} \\
{[\mathbf{k H z}]}\end{array}$ & $\begin{array}{c}\boldsymbol{L}_{\boldsymbol{r}} \\
{[\mathbf{c m}]}\end{array}$ & $\begin{array}{c}\boldsymbol{L}_{\boldsymbol{R}} \\
{[\mathbf{c m}]}\end{array}$ & $\begin{array}{c}\boldsymbol{L}_{\boldsymbol{A}} \\
{[\mathbf{m}]}\end{array}$ & $\begin{array}{c}\boldsymbol{L}_{\boldsymbol{s}} \\
{[\mathbf{m}]}\end{array}$ & $\begin{array}{c}\boldsymbol{t}_{\text {vuelo }} \\
{[\boldsymbol{\mu s}]}\end{array}$ & $\begin{array}{c}\boldsymbol{t}_{\text {refl }} \\
{[\boldsymbol{\mu s}]}\end{array}$ & $\begin{array}{c}\Delta \boldsymbol{t}_{\text {rec }} \\
{[\boldsymbol{\mu s}]}\end{array}$ \\
\hline Airmar P19 & 200 & 8.6 & 27.61 & 136 & 180.40 & 0.24 & 0.84 & 0.6 \\
\hline
\end{tabular}

Tabla 1.1: Distancias características del transductor proyector empleado en las medidas experimentales. $t_{r e f l}$ es el tiempo de vuelo de la primera reflexión. La distancia entre emisor y receptor es de $35 \mathrm{~cm}$

\subsubsection{Comparación teórica y experimental con el modelo de Moffett y Mellen}

Una vez se tienen en cuenta las distancias anteriores, se emplean modelos matemáticos que ayudarán a predecir el comportamiento del campo acústico de las frecuencias secundarias [19]. Para ello, se tiene en cuenta que existen 4 regímenes operativos para las fuentes acústicas paramétricas, descritas a continuación.

- Limite de Absorción: La onda inicial se absorbe en el campo cercano del transductor sin llegar a formar onda de choque, lo que permite que, la zona de interacción no lineal está constreñida, y se genere un campo no lineal ancho. Westervelt.

- Limite de Absorción y difración: No se llega a formar del todo la onda de choque. Lo que genera escasos efectos no lineales. Saturación. 
- Limite de Choque y difracción: Se llega a formar la onda de choque pero dentro del campo cercano del transductor, lo que genera efectos no lineales limitados. Moffett y Mellen.

- Limite Difracción: Las no linealidades se propagan más allá del campo cercano, por lo tanto, la zona de interacción no lineal es más extensa, lo que genera un campo no lineal directivo. Berktay y Leahy.

En el diseño de fuentes paramétricas, es necesario poder predecir el rendimiento de las fuentes que operan entre estos cuatro extremos. Varios autores han tomado pasos en esta dirección, entre ellos, Mellen y Moffett recurriendo a un modelo simplificado del campo del pistón que incluye como casos limitantes los cuatro regímenes operativos enumerados anteriormente. Sus resultados se trazan en las curvas de diseño, presentadas en [20] que permiten un cálculo rápido de las características esenciales de la frecuencia diferencia para compararlos con datos obtenidos experimentalmente.

\section{Desarrollo teórico y experimental}

En este apartado se compara la relación de amplitud entre el haz secundario y el haz primario $A_{s} / A_{p}$, obtenido experimentalmente con el modelo teórico al que se hace referencia en [20] y [21]. Este estudio se análisis de dos formas; en la primera, se estudian las presiones recibidas del haz primario y secundario y la segunda, a través de las frecuencias emitidas.

Con las presiones en pascales medidas experimentalmente para el haz primario de $12943 \mathrm{~Pa}$ y el secundario de $36.8 \mathrm{~Pa}$, se estima un $0.28 \%$ de relación directa.

Se realiza un primer análisis utilizando la ecuación de la ganancia paramétrica compleja $G$, que se define como un valor cuya magnitud es la relación entre las presiones del haz primario y secundario:

$$
g \equiv r P(r, 0) / R_{o} P_{o}
$$

en decibeles,

$$
G=20 \log |g|
$$

Donde $r$, es la coordenada radial del observador, $P(r, 0)$ es la presión del haz secundario en un punto $r$ centrado al emisor en $P a, R_{o}=\left(A_{o} f_{o}\right) / c$, es la distancia de Rayleigh en $m$, y $P_{o}$, es la presión del haz primario a la distancia de Rayleigh. 
De acuerdo con las calibraciones realizadas, para el caso estudiado en cuestión, $g \approx$ 0.028 y G $\quad-51 \mathrm{~dB}$ obteniendo así una relación de amplitud entre el haz secundario y primario de $0.28 \%$. La ganancia $G$ tiene un valor de $L_{o}^{*}=245 \mathrm{~dB} \mathrm{rms}$ del nivel de la fuente primaria y $\bar{\alpha} R_{o} \approx 10^{-3} \mathrm{~dB}(0.0012 \mathrm{~dB})$.

$$
L_{o}=20 \log \left(\frac{P_{o} R_{o}}{\sqrt{2}}\right) \quad y \quad L_{o}^{*}=L_{o}+20 \log f_{o} \quad[d B / / 1 \mu P a \mathrm{~m} \mathrm{kHz}]
$$

Siguiendo las curvas de ganancia paramétricas, se tiene que, efectivamente los resultados experimentales obtenidos se encuentran cerca a los valores de $10^{-3}$ para $\bar{\alpha} R_{o}$ y $L_{o}^{*} \sim 240$. Como se puede observar en la siguiente Figura 1.19.

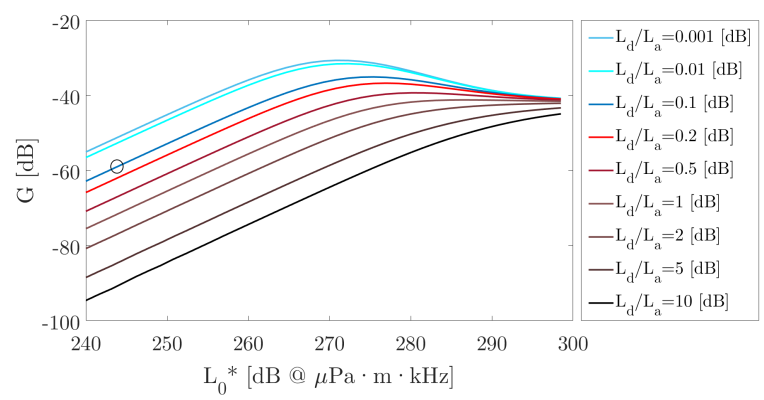

Figura 1.19: Curva de ganancia paramétrica del modelo Moffett y Mellen

El segundo análisis consiste en aplicar la ecuación (1.127), se estudia el sweep medido experimental desde los 4 hasta los $40 \mathrm{kHz}$, como un conjunto de senos paramétricos en las frecuencias límites y una centrada; es decir, las frecuencias de $4 \mathrm{kHz}, 20$ $\mathrm{kHz}$ y $40 \mathrm{kHz}$ (que serán estudiadas en los próximos capítulos). Las relaciones entre el haz secundario y el haz primario obtenidos son $0.141 \%, 0.35 \%$ y $1.41 \%$, respectivamente, con una presión del haz primario de $12940 \mathrm{~Pa}$. La relación más cercana a la experimental es la de onda senoidal de $20 \mathrm{kHz}$, que es aproximadamente la frecuencia promedio del sweep paramétrico medido experimentalmente.

Finalmente, la medición también se contrasta de acuerdo con el régimen de operación para fuentes paramétricas. Para este caso, se encuentra en el régimen del límite de absorción en la zona esférica del campo lejano, siguiendo la referencia [20]:

$$
X \ll 1, \quad 2 \alpha R_{o} f_{o} / f \ll 1
$$

donde la absorción $\alpha$ se expresa en $\left(N_{p} / m\right), R_{o}$ en m; que es la distancia de Rayleigh, $f_{o}$ en $\mathrm{Hz}$ es la frecuencia portadora y $f$, es la frecuencia diferencia para 4,20 y $40 \mathrm{kHz}$. 
Aplicando la ecuación (1.131), se obtiene 0.014, 0.0028 y 0.0014 respectivamente, y para $X 0.006$, se tiene que, todos los valores son mucho mas pequeños que la unidad (1).

Adicionalmente, el parámetro $g$ se calcula a través de la siguiente ecuación:

$$
|g|=\frac{X}{2} \frac{f}{f_{o}} E_{1}\left(\frac{2 \alpha R_{o} f_{o}}{f}\right)
$$

donde $E_{1}$ es la función integral exponencial. Se obtiene 0.0002 para $4 \mathrm{kHz}, 0.0015$ para $20 \mathrm{kHz}$ y 0.0035 para $40 \mathrm{kHz}$ y aplicando la eq 1.129 , se tiene $-73.36 \mathrm{~dB}-56.27$ $\mathrm{dB} y-49.18 \mathrm{~dB}$, respectivamente, validando que para la frecuencia emitida de $20 \mathrm{kHz}$ el valor de $\mathrm{G} \quad-56.27 \mathrm{~dB}$ es similar al primer análisis que estudia la presión recibida de los haces primario y secundario con $\mathrm{G} \quad-51 \mathrm{~dB}$.

\subsection{Introducción a la acústica submarina}

La acústica submarina estudia la interacción y la propagación de las ondas acústicas en el agua, siendo un canal complicado, ya que por naturaleza es un medio no homogéneo debido, a las variaciones de la velocidad del sonido con la profundidad que conduce en sí, a la formación del canal submarino y como consecuencia a la propagación. En los siguientes apartados, se exponen aquellas contribuciones que permiten entender el comportamiento del canal acústico en general, y en especial en aguas poco profundas.

\subsubsection{Fenomenología del canal acústico submarino}

Una onda sonora, se genera debido a una perturbación mecánica que se propaga en un medio elástico con una determinada velocidad, según las características del medio. Parte de la energía mecánica de esta onda, se absorbe por el medio en el que se propaga debido principalmente al rozamiento y a fenómenos resonantes.

La transmisión de información en el canal submarino se basa en técnicas acústicas, ya que la absorción de estas señales es mucho menor a diferencia de las señales de radiofrecuencia o las ópticas, como se estudiará en el Capítulo 2.

Las características químicas y físicas del medio marino afectan a la propagación de la señal acústica debido a fenómenos tales como: velocidad de propagación de la señal acústica que varía con la profundidad del agua y las pérdidas por transmisión $\left(\mathrm{TL}^{1}\right)$,

\footnotetext{
${ }^{1}$ Transmission Loss
} 
debida a la dispersión geométrica y a la absorción. Además, se tiene el ruido ambiental, causado por los organismos marinos, tráfico de los barcos, entre otros.

Todos los anteriores fenómenos que afectan a la propagación de la señal acústica en el canal submarino, determinan la amplitud de la señal recibida y en consecuencia el desempeño del receptor [22].

A continuación, se presenta una descripción general de estos parámetros que intervienen en la propagación de una señal acústica y que son relevantes en el diseño de sistemas de comunicación.

\section{Velocidad de propagación en el mar}

La velocidad de propagación de las ondas acústicas en el agua de mar se puede considerar de $1500 \mathrm{~m} / \mathrm{s}$, sin embargo, esta varía entre $1450 \mathrm{~m} / \mathrm{s}$ y $1540 \mathrm{~m} / \mathrm{s}$ [23] debido a la temperatura, salinidad y presión del agua. A continuación, se explica la influencia de estos parámetros en la velocidad de propagación para, posteriormente, estudiar la influencia de la profundidad y asi obtener el perfil de velocidad.

- Temperatura: en general, la temperatura del agua de mar disminuye desde la superficie hasta el fondo, siendo máxima en las capas poco profundas debido al calentamiento solar [24,25]. Más allá de una profundidad límite (alrededor de 1000 $\mathrm{m}$ en mar abierto) la temperatura promedio permanece estable, disminuyendo muy lentamente con la profundidad y variando poco de un lugar a otro.

- Presión: La presión hidrostática hace que la velocidad de propagación de las ondas acústicas aumente con la profundidad debido a las variaciones del módulo de elasticidad.

- Salinidad: la masa porcentual de la salinidad se conforma por una mezcla de agua pura y sales disueltas ( $\mathrm{NaCl}, \mathrm{MgSO} 4$, entre otras). El promedio de la salinidad del agua del mar es de unos 35 ppt (partes por mil). La variación de la salinidad respecto a la profundidad es mínima y por lo tanto se desprecia, excepto en aquellos lugares polares.

De entre las diferentes expresiones existentes para obtener la velocidad de propagación de las ondas acústicas en el agua de mar [26-30], en esta tesis se emplea la expresión de Medwin [29] y la de Mackenzie [30], cada una de ellas válida para un rango de profundidades.

- Para profundidades menores a $1000 \mathrm{~m}$ se tiene la expresión de Medwin con temperaturas entre $0<T<35^{\circ} \mathrm{C}$ y salinidad $S<45$ ppt: 


$$
c=1449.2+4.6 T-0.055 T^{2}+0.00029 T^{3}+(1.34-0.01 T)(S-35)+0.016 z
$$

- Para profundidades mayores a $1000 \mathrm{~m}$ se tiene la expresión de Mackenzie, con temperaturas entre $0<T<30^{\circ} \mathrm{C}$ y salinidad entre $30<S<40 \mathrm{ppt}$ :

$$
\begin{gathered}
c=1448.96+4.591 T-5.304 \cdot 10^{-2} T^{2}+2.374 \cdot 10^{-4} T^{3} \\
+1.340(S-35)+1.630 \cdot 10^{-2} z+1.675 \cdot 10^{-7} z^{2} \\
-1.025 \cdot 10^{-2} T(S-35)-7.139 \cdot 10^{-13} T z^{3} \\
p^{\prime}(0, t)=A_{p} \cos \left(\omega_{m} t\right) \sin \left(\omega_{p} t\right) \\
=\frac{A_{p}}{2} \sin \left(\omega_{p}+\omega_{m}\right) t+\frac{A_{p}}{2} \sin \left(\omega_{p}-\omega_{m}\right) t \\
=\mathcal{P}_{o} \sin \omega_{a} t+\mathcal{P}_{o} \sin \omega_{b} t
\end{gathered}
$$

Donde $c$ es la velocidad del sonido en $m / s, T$ es la temperatura en ${ }^{\circ} C, z$ es la profundidad en $m$, y $S$ es la salinidad en $p p t$.

\section{Perfil de velocidad}

Como se vio, la velocidad de propagación depende de la temperatura, salinidad y presión, las cuales varían dependiendo del lugar, la época del año, y la hora. Sin embargo, al analizar el perfil de velocidad, los cambios vienen dados por la variación de la presión con la profundidad, ya que, a profundidades mayores, la temperatura es constante y la salinidad varía mínimamente.

El perfil de velocidad descrito a continuación y presentado en la Figura 1.20, es el perfil típico para latitudes medias con valores de profundidad dados en metros que se emplea como referencia en muchas ocasiones [9]. A continuación, se describen las capas características que lo componen.

- Capa homogénea, en los primeros metros de profundidad, denominada aguas muy poco profundas, se tiene una velocidad de propagación que permanece constante.

- Capa superficial, está formada por el conjunto de aguas poco profundas hasta los 200 metros, donde las ondas acústicas tienden a ser atrapadas hacia arriba por 
las reflexiones de la superficie. La velocidad de propagación aumenta debido a los cambios de temperatura del agua, la estación del año y la hora del día, entre otros factores.

- Capa termoclina principal, está presente entre los 200 a 1000 metros. En esta capa, la velocidad del sonido disminuye con la profundidad al disminuir también la temperatura.

- Capa isotérmica profunda, desde los 1000 metros en adelante, la temperatura es casi constante, aproximada a los $4^{\circ} \mathrm{C}$ hasta el fondo del mar y la velocidad del sonido se mantiene casi constante.

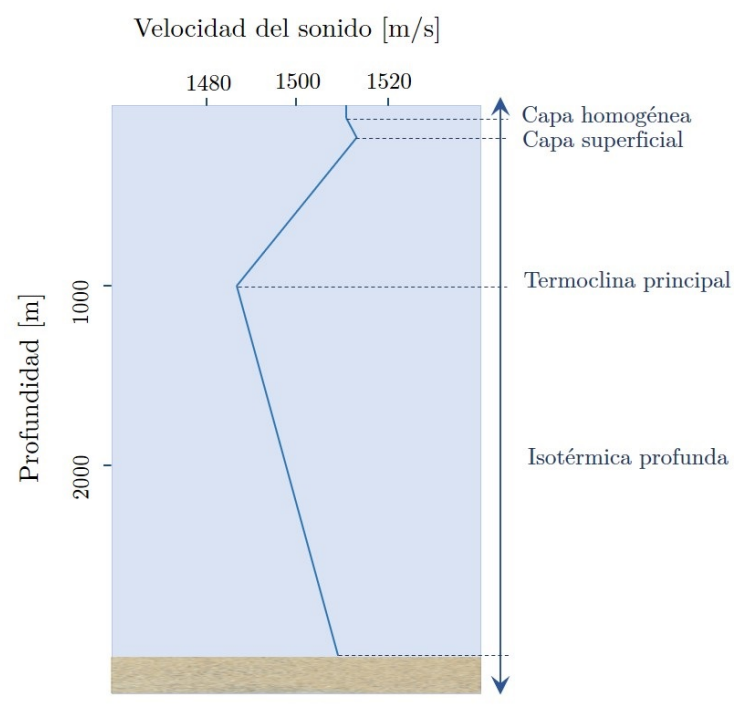

Figura 1.20: Perfil típico de la velocidad del sonido en función de la profundidad

\section{Absorción}

La absorción se produce cuando parte de la energía de la señal acústica se transforma en calor, debido a la disipación por rozamiento viscoso y reacciones químicas. En el agua de mar, hay dos procesos de relajación química que tienen una gran importancia en la contribución a la absorción: los que implican ácido bórico $B\left(\mathrm{OH}_{3}\right)$ y los relacionados con el sulfato de magnesio $\mathrm{Mg}\left(\mathrm{SO}_{4}\right)$ [31].

Si bien, la frecuencia es la principal variable en la absorción, esta última también depende del medio de propagación, es decir, la temperatura del agua y la salinidad. Las 
formulaciones para predecir el coeficiente de absorción a una frecuencia, temperatura, salinidad y profundidad determinadas, fueron desarrolladas por diversos autores de forma semiempírica, entre ellos, Marsh-Schulkin y Fisher \& Simmons; [32, 33]. Con todo, el más utilizado es el formulado por Francois \& Garrison [34, 35] en 1982 que sostienen que, asumiendo el conocimiento exacto de la temperatura y otros parámetros, el coeficiente de absorción $(\alpha)$ predicho, estará dentro del $5 \%$ del valor verdadero. Por ello, de entre los diferentes métodos para estudiar la absorción, en esta tesis se emplea el modelo de Francois \& Garrison. Así, la absorción total de estas contribuciones se expresa como sigue [36]:

$$
\alpha=A_{1} P_{1} \frac{f_{1} f^{2}}{f_{1}^{2}+f^{2}}+A_{2} P_{2} \frac{f_{2} f^{2}}{f_{2}^{2}+f^{2}}+A_{3} P_{3} f^{2}
$$

Donde $\alpha$ es la atenuación en $\mathrm{dB} / \mathrm{km}, A_{i}$ y $P_{i}$ son los coeficientes empíricos y $f$ es la frecuencia de la señal, expresada en $\mathrm{kHz}$. En esta expresión, la primera parte de la ecuación corresponde al ácido bórico, la segunda al sulfato de magnesio y la última a la viscosidad del agua. Además, la absorción depende en gran medida de la frecuencia, y aumenta para las frecuencias más altas.

A continuación, se presentan cada una de las diferentes contribuciones que, en conjunto, expresan la fórmula de la absorción presentada en (1.136). En general, cada una de las contribuciones hacen parte de la relajación molecular que consiste en la disociación de algunos compuestos iónicos en soluciones $\mathrm{B}(\mathrm{OH})_{3}, \mathrm{Mg}(\mathrm{SO})_{4}$, debido a las variaciones de presión locales creadas por la onda acústica. Este proceso es dominante para la absorción de la onda acústica en el agua de mar.

- Contribución de ácido bórico $B(O H)_{3}$ :

$$
\begin{aligned}
& A_{1}=\frac{8.86}{c} 10^{0.78 p H-5} \\
& P_{1}=1 \\
& f_{1}=2.8 \sqrt{\frac{S}{35} 10}\left(4-\frac{1245}{T+273}\right)
\end{aligned}
$$

- Contribución de sulfato de magnesio $\mathrm{Mg}(\mathrm{SO})_{4}$ : 


$$
\begin{aligned}
& A_{2}=21.44 \frac{S}{c}(1+0.025 T) \\
& P_{2}=1-1.37 \cdot 10^{-4} z+6.2 \cdot 10^{-9} z^{2} \\
& f_{2}=\frac{8.17 \cdot 10^{\left(8 \frac{1990}{T+273}\right)}}{1+0.0018(S-35)}
\end{aligned}
$$

- Viscosidad del agua:

$$
\begin{aligned}
T \leq 20^{\circ} \mathrm{C} \quad P_{3}= & 1-3.83 \cdot 10^{-5} z+4.9 \cdot 10^{-10} z^{2} \\
A_{3}= & 4.937 \cdot 10^{-4}-2.59 \cdot 10^{-5} T+9.11 \cdot 10^{-7} T^{2} \\
& -1.5 \cdot 10^{-8} T^{3} \\
T>20^{\circ} \mathrm{C} \quad A_{3}= & 3.964 \cdot 10^{-4}-1.146 \cdot 10^{-5} T+1.45 \cdot 10^{-7} T^{2} \\
& -6.5 \cdot 10^{-10} T^{3}
\end{aligned}
$$

En la Figura 1.21, se presenta el coeficiente de absorción con las condiciones promedio del Mar Mediterráneo como son: $T=10^{\circ}$ a $20^{\circ} \mathrm{C}, S=37 p p t$ y $p H=8$ empleando la ecuación de Francois \& Garrison.

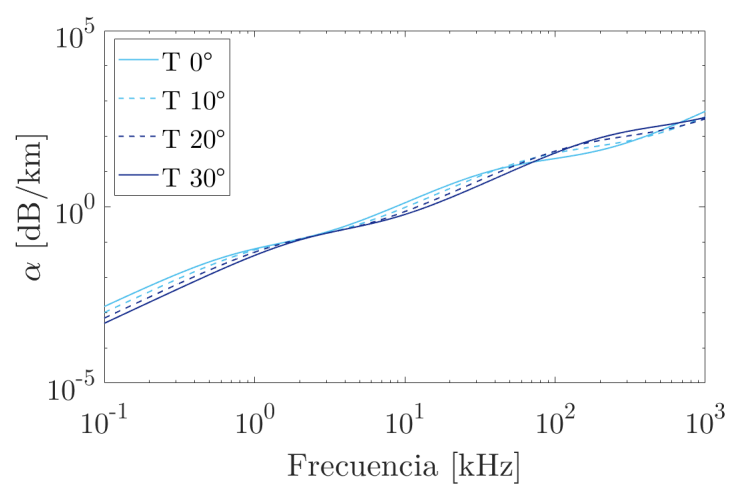

Figura 1.21: Coeficiente de absorción en el mar, en función de la frecuencia con una salinidad de 35 p.s.u. con diferentes valores de temperatura

En ella, se observa el aumento de la absorción con la frecuencia. Es decir, que si se desea transmitir señales a grandes distancias del orden de los cientos de $(\mathrm{km})$ se tendrán que emplear frecuencias de decenas de $\mathrm{kHz}$ ya que si suelen ser más altas el medio las atenuará. Pero si se quieren enviar señales a distancias cortas, del orden de los $(\mathrm{m})$, se pueden enviar frecuencias de cientos de kHz. Además, para las frecuencias 
menores a $1 \mathrm{kHz}$ la absorción es menor. A $100 \mathrm{kHz}$, el coeficiente de absorción está alrededor de las decenas de $\mathrm{dB} / \mathrm{km}$.

\subsubsection{Propagación en aguas poco profundas}

\section{Efecto multicamino}

Como se comentó en el apartado anterior, en la capa superficial o aguas poco profundas la propagación de la onda acústica está limitada por la superficie del mar y el lecho marino, lo que permite que, las señales transmitidas sufran sucesivas reflexiones en estas superficies. Así, una señal puede propagarse desde la fuente al receptor a través de diferentes caminos, que corresponden a varias direcciones y duraciones. Con esto, la señal recibida constará de la primera señal que llegue, o señal directa, junto con una serie de señales adicionales retardadas con distinta amplitud. Entonces, el perfil temporal de la señal registrada en el receptor variará notablemente de la señal enviada por la fuente.

En general, este efecto es notorio para transductores proyectores omnidireccionales, como son los transductores de baja frecuencia, típicamente utilizados en aplicaciones de acústica submarina. Con el uso de sistemas directivos se pueden evitar cierta influencia del efecto multicamino y, con ello, mejorar la calidad de la señal recibida.

\section{Modelado de la propagación}

A pesar de que existen modelos robustos para la simulación de la propagación acústica de las aguas en el mar, existen modelos simplificados que abordan la propagación en aguas poco profundas como el modelo semiempírico de Colossus o también llamado Marsh-Schulkin (M-S) [ [32], con un nivel de aproximación suficiente para los propósitos de esta tesis. En él, se estudia la pérdida de propagación en términos de la altura de la ola, tipo de fondo, profundidad del agua, frecuencia y perfil de velocidad. Este último, está conformado por dos segmentos; el primero, se extiende desde la superficie del mar hacia una profundidad $L$ dada en metros, donde la velocidad de propagación aumenta con la profundidad y el segundo, para profundidades mayores que $L$, donde la velocidad disminuye con la profundidad.

En función de la profundidad de la capa superficial y la profundidad del agua, se formula una única distancia efectiva de salto o distancia de transmisión $H$, dada en (1.140). Donde $D$, es la profundidade del agua y $L$, es la profundidad de la capa isotérmica mixta, ambas en metros. 


$$
H=\left(\frac{L+D}{3}\right)^{1 / 2}
$$

La distancia de salto se usa como una referencia para definir las regiones de propagación del frente de onda. Se tiene entonces lo siguiente:

Para rangos de $R<H$, la zona de separación de rayos directos.

$$
T L=20 \log R+\alpha R+60-k_{L}
$$

Para rangos de $H<R<8 H$, la zona de separación de modos.

$$
T L=15 \log R+\alpha R+60+\alpha_{T}\left(\frac{R}{H}-1\right)+5 \log H+60-k_{L}
$$

Para rangos de $R>8 H$, zona de control de modo único.

$$
T L=10 \log R+\alpha R+\alpha_{T}\left(\frac{R}{H}-1\right)+10 \log H+64.5-k_{L}
$$

Donde $R$ se expresa en $\mathrm{km}, \alpha$ es el coeficiente de absorción en $\mathrm{dB} / \mathrm{km}, \alpha_{T}$ es el coeficiente de atenuación efectivo, que tiene en cuenta las pérdidas debidas al acoplamiento de energía entre la superficie y el fondo, dado en $\mathrm{dB} /$ rebote, $k_{L}$ es el paramétro al que se le denomina anomalía de campo cercano y mide la ganancia dada por los rebotes entre el fondo marino y su superficie, dado en $\mathrm{dB}$.

Estas ecuaciones proporcionan una transición gradual de la propagación esférica en el campo cercano hasta la propagación cilíndirca en campo lejano.

A continuación, en la Figura 1.22, se muestra un ejemplo de propagación a 50 m y 200 $\mathrm{m}$, en aguas poco profundas entre la superficie y el lecho marino para una frecuencia de $1 \mathrm{kHz}$ y $10 \mathrm{kHz}$ respectivamente, según el modelo de Marsh-Shulkin para tres los rangos presentados.

En esta figura, se tiene que los últimos dos rangos son parecidos entre ellos para ambos ejemplos, mientras que, para el primer rango se tiene una diferencia de aproximadamente $40 \mathrm{~dB}$ respecto de los dos últimos. 

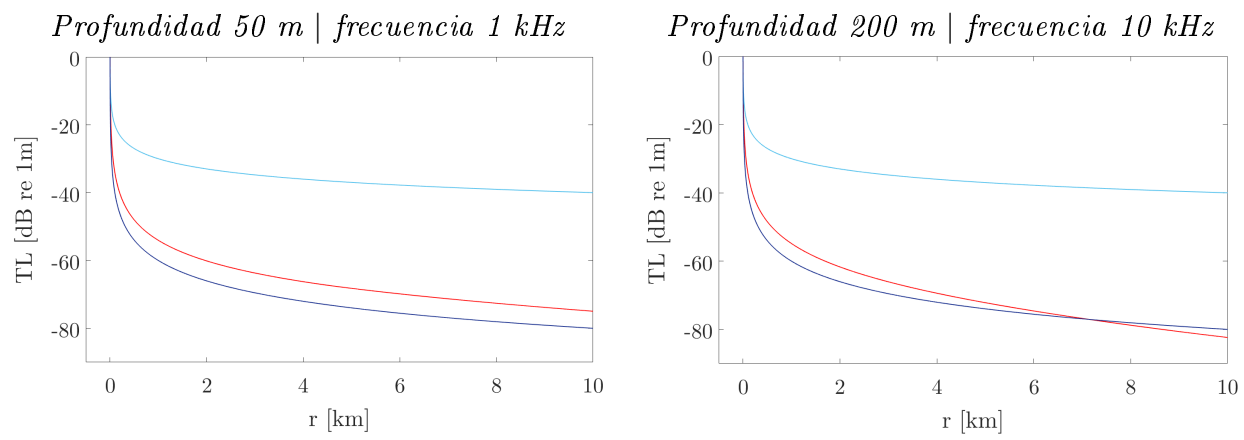

Figura 1.22: Ejemplo de propagación según modelo de Mars-Shulkin para diferntes rangos. (azul claro) TL cilíndrica, (roja) TL en zona de separación de modos y (azul oscuro) TL esférico

\subsubsection{Consideraciones sobre el ruido de fondo}

Se debe tener en cuenta que los conceptos de sonido, señal y ruido son diferentes: el sonido es un término que se refiere a cualquier energía acústica; el ruido es un subconjunto de sonidos no deseados por un oyente y tiende a ser subjetivo. Ahora bien, todo sonido sobre el que es requerido realizar una observación se califica como señal, por lo tanto, cualquier sonido específico puede ser una señal para algunos y un ruido para otros [37].

El ruido de fondo o ruido ambiente se debe al sonido proveniente de aquellas fuentes artificiales originadas por el hombre y al ruido generado intrínsecamente por las fuentes naturales. Así, se tienen el ruido de las olas de mar, gotas de lluvia, perturbaciones, tráfico marítimo, perforaciones, entre otros.

Para saber los niveles de ruido ambiental en una banda de frecuencia y condiciones específicas. Se utilizan las curvas de Wenz [38] que es una gráfica que describe el espectro del ruido de fondo o ambiental promedio para diferentes condiciones del clima, actividad geológica, tráfico de embarcaciones y velocidades del viento [39].

La Figura 1.23, muestra la densidad espectral de potencia del ruido submarino en en diferentes frecuencias dadas por Wenz.

De entre los ruidos predominantes en este estudio de Wenz, se tienen lo siguiente:

- Por debajo de $10 \mathrm{~Hz}$ se tiene que la principal fuente de ruido estacionario es el fondo sísmico y las señales transitorias se deben a explosiones o terremotos. 
- Entre $10 \mathrm{~Hz}$ y $1 \mathrm{kHz}$, el ruido difuso se debe al tráfico oceánico y a los transitorios del movimiento del barco.

- Entre los $1 \mathrm{kHz}$ y $40 \mathrm{kHz}$ se tiene que es la región más importante y variable del espectro. La principal contribución a la señal aleatoria se debe a las interacciones del viento y la lluvia con la superficie del mar estando vinculada a las condiciones climáticas.

En esta sección, se encuentran en su mayoría las señales acústicas con las que se trabajan en esta tesis. La fuente de ruido ambiental dominante generalmente es el ruido de la superficie generado por el clima. Esto es lo suficientemente significativo como para que el rendimiento del sistema acústico cambie con las condiciones climáticas. Sobre los $40 \mathrm{kHz}$, se cree que la principal contribución proviene del ruido térmico de las moléculas de agua. 


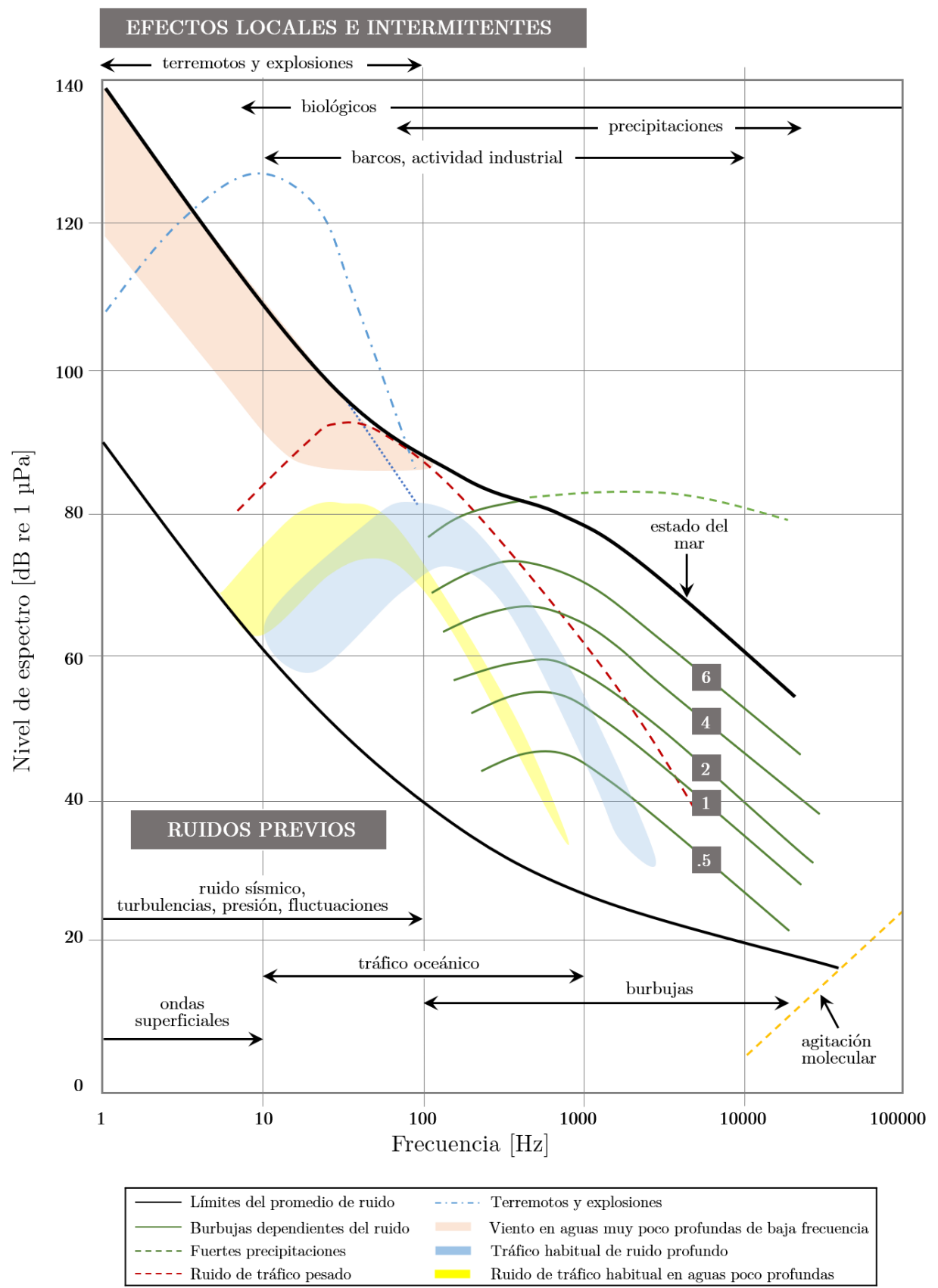

Figura 1.23: Gráfica de Wenz que representa los niveles sonoros del ruido de fondo del mar en diferentes rangos frecuenciales 



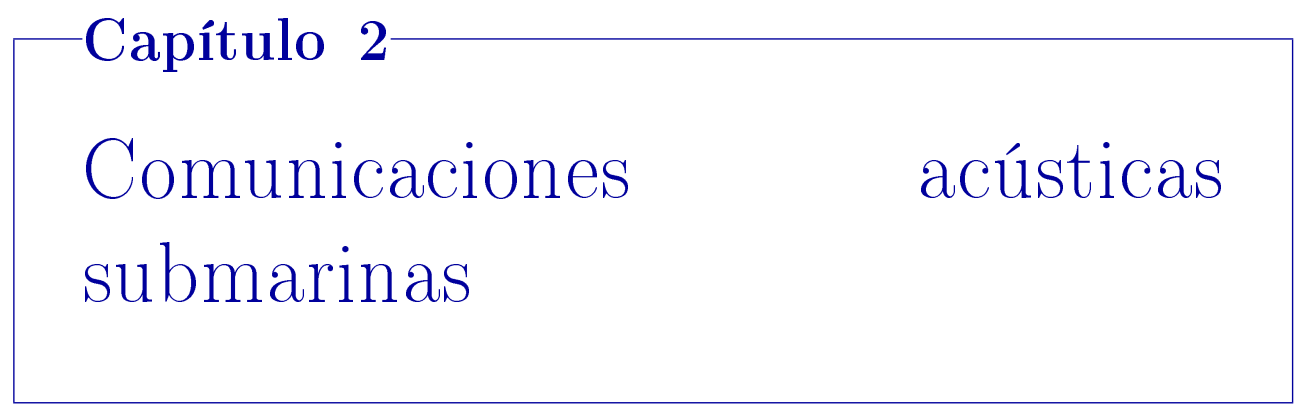

El agua es un medio natural para la propagación de las ondas acústicas, por lo que hoy en día estas son un campo de investigación en diversas aplicaciones, entre ellas, las comunicaciones submarinas. En este capítulo, se estudian las técnicas de modulación empleadas en las comunicaciones acústicas submarinas para así utilizarlas en el fenómeno de propagación no lineal llamado, efecto paramétrico.

En el apartado 2.1, se presenta una breve descripción comparativa entre las diferentes tecnologías existentes en las comunicaciones submarinas: las de radiofrecuencia, las ondas ópticas y las ondas acústicas; con el fin de justificar las ventajas y desventajas de las tecnologías acústicas. En el apartado 2.2, se expone una revisión histórica de las comunicaciones acústicas submarinas que incluye los avances en las diferentes técnicas de modulación para la transmisión digital de información, así como sus aplicaciones. Además, se presenta un compendio de investigaciones realizadas por otros investigadores relacionado con el efecto paramétrico aplicado a las comunicaciones acústicas submarinas.

En el apartado 2.3, se introducen las diferentes técnicas de modulaciones, tanto analógicas como digitales. Con ello y haciendo referencia a los resultados del Capítulo 1, en el apartado 2.4 se expone la técnica para obtener las correspondientes modulaciones para comunicación acústica basada en el efecto paramétrico que, en última instancia, consiste en las combinaciones de tonos (senos) o sweeps (barridos senoidales). Se aborda el método directo, que obtiene la nueva modulación a través de la aplicación directa de la técnica de demodulación de Berktay a la modulación lineal. 


\subsection{Las comunicaciones submarinas}

Las comunicaciones submarinas consisten en la transmisión de información a través del mar mediante la utilización de tecnologías basadas en ondas de radiofrecuencia, ondas ópticas o acústicas; cada una de estas tecnologías presentan ciertas limitaciones y ventajas. En comparación con las comunicaciones aéreas, presentan la limitación de que el agua es opaca a la radiación electromagnética, excepto en la banda visible e incluso, en esta banda la luz penetra sólo unos pocos cientos de metros en aguas claras. En consecuencia, las tecnologías acústicas han tomado importancia en el desarrollo de sistemas de comunicaciones submarinas hasta llegar, actualmente, a representar una tecnología relativamente madura y robusta a pesar de sus limitaciones en cuanto a tasa de transmisión de datos y latencia [40,41]. En este apartado, se explica brevemente la influencia del canal submarino en las diferentes tecnologías existentes para la transmisión de información, y así entender este auge en las tecnologías acústicas.

\subsubsection{Comunicaciones por radiofrecuencia}

Uno de los mayores logros tecnológicos de la historia moderna, es la implementación de medios de comunicación y transmisión de información a través de largas distancias (teléfono, radio, televisión). La mayoría de estos sistemas usan ondas electromagnéticas, de naturaleza similar a la luz, cuyas propiedades se descubrieron en el siglo XIX y se utilizaron por primera vez a principios del siglo XX. Desde entonces, han tenido un papel importante para la transmisión de información [9]. Sin embargo, una gran parte de la Tierra sigue siendo inaccesible a las ondas electromagnéticas; el reino submarino, que representa más del $70 \%$ de su superficie, no permite la propagación de ondas de radiofrecuencia adecuadamente. En efecto, dado que el agua de mar, presenta una fuerte conductividad ( $4 \mathrm{~S} / \mathrm{m}$ en promedio), que aumenta sobre todo con la salinidad, esto se traduce en una mayor atenuación $(\sim 1 \mathrm{~dB} / \mathrm{m})$, lo que limita el alcance y utilidad de las comunicaciones submarinas por radiofrecuencia $[42,43]$.

Ahora bien, como la atenuación aumenta con la frecuencia tal como se observa en la Figura 2.1 las comunicaciones electromagnéticas se podrían emplear con bajas frecuencias en agua dulce donde hay menor conductividad, mejorando su alcance. Sin embargo, esto precisaría, de antenas más grandes debido a las mayores longitudes de onda de estas frecuencias. 


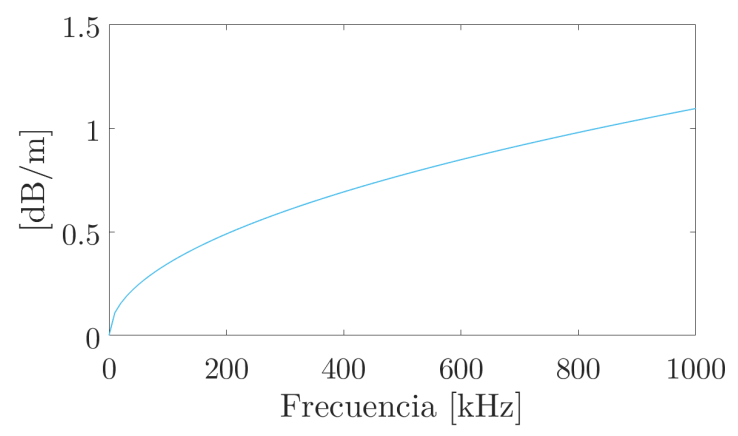

Figura 2.1: Absorción de ondas por radiofrecuencia

\subsubsection{Comunicaciones ópticas}

Las comunicaciones ópticas emplean ondas electromagnéticas con longitudes de onda entre los $400 \mathrm{~nm}$ y $500 \mathrm{~nm}$, presentando una gran ventaja en la transmisión de datos, que potencialmente, puede superar $1 \mathrm{Gbps}$. Sin embargo, existen desventajas para la comunicación óptica en el agua, tales como: las señales ópticas se absorben rápidamente en el agua de modo, que la luz penetra solo unos pocos cientos de metros en las aguas más claras y mucho menos en aguas turbias; las partículas en suspensión ocasionan una dispersión óptica significativa, el alto nivel de luz ambiental en la parte superior de la columna de agua interfiere en la comunicación [44].

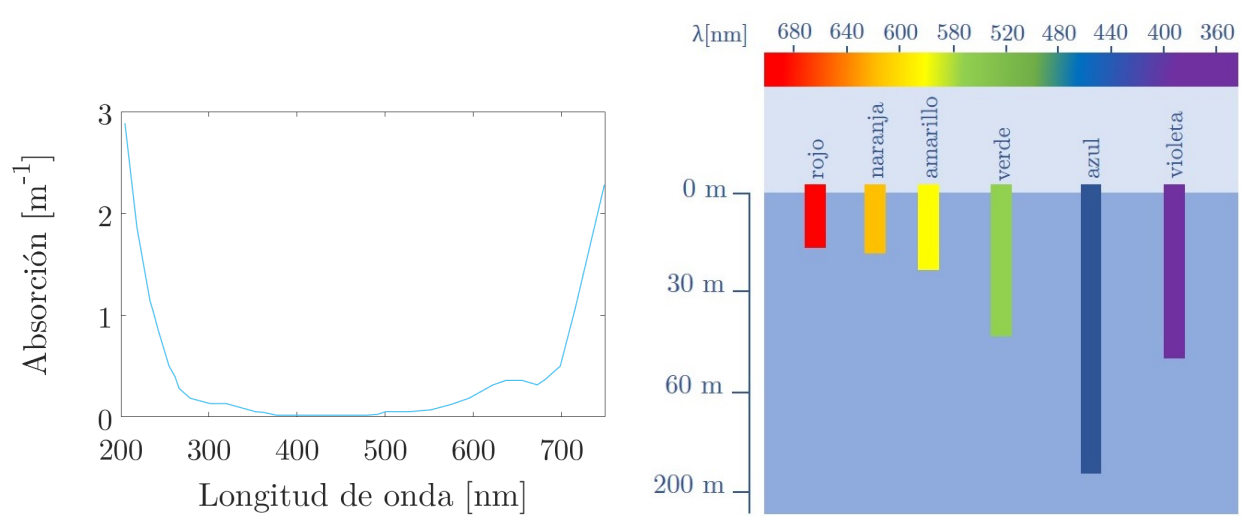

Figura 2.2: Gráfica de la absorción de la luz en el agua de mar. Espectro visble (izquierda), penetración (derecha)

En la Figura 2.2 se muestra la curva de absorción del agua de mar para el espectro visible (izquierda), así como su penetración (derecha). Se observa que la radiación 
roja, naranja y amarilla se atenúan rápidamente, entre los primeros $30 \mathrm{~m}$, mientras que las longitudes de onda entre 400 y $500 \mathrm{~nm}$ (radiación verde azulada) presentan, una menor absorción y mayores longitudes de penetración.

\subsubsection{Comunicaciones acústicas}

Las ondas acústicas consisten en vibraciones mecánicas que se propagan en un medio. Estas, se pueden desplazar fácilmente en el agua de mar, donde su uso podría compensar hasta cierto punto las capacidades limitadas de las ondas de radiofrecuencia y ópticas en este medio.

Las ondas acústicas en el agua se propagan mucho mejor que en el aire (su velocidad de propagación es cuatro veces mayor), pueden alcanzar niveles mucho más elevados y sobre todo, sufren menos atenuación en comparación con los sistemas de comunicación anteriormente descritos $(\sim 1 \mathrm{~dB} / \mathrm{km})$; por lo tanto, pueden viajar a grandes distancias y penetrar a mayores profundidades [45]. Estas ventajas se ven mitigadas por otras limitaciones como, la perturbación por un nivel de ruido ambiental más alto, y reflexiones no deseadas que aprovechan las mismas condiciones para una fácil propagación.

\subsubsection{Comparativa}

A modo de resumen, se presenta la Tabla 2.1 que aúna las características de las tecnologías empleadas en las comunicaciones submarinas.

\begin{tabular}{llll}
\hline & Acústica & Radiofrecuencia & Óptica \\
\hline Velocidad del sonido & $\sim 1500 \mathrm{~m} / \mathrm{s}$ & $\sim 300000 \mathrm{~km} / \mathrm{s}$ & $\backsim 300000 \mathrm{~km} / \mathrm{s}$ \\
Velocidad de datos & $<100 \mathrm{Kbps}$ & $<10 \mathrm{Mbps}$ & $<1 \mathrm{Gbps}$ \\
Pérdidas de potencia & $>0.1 \mathrm{~dB} / \mathrm{m} / \mathrm{Hz}$ & $\sim 28 \mathrm{~dB} / \mathrm{km} / 100 \mathrm{MHz}$ & turbidez \\
Ancho de banda & $\sim \mathrm{kHz}$ & $\sim \mathrm{MHz}$ & $\backsim 10-150 \mathrm{MHz}$ \\
Banda frecuencial & $\sim \mathrm{kHz}$ & $\sim \mathrm{MHz}$ & $\sim 10^{14}-10^{15} \mathrm{~Hz}$ \\
Limitaciones & Ancho banda & Potencia & Ambiente \\
Rango efectivo & $\sim \mathrm{km}$ & $\sim 1-100 \mathrm{~m}$ & $\sim 1-100 \mathrm{~m}$ \\
\hline
\end{tabular}

Tabla 2.1: Comparación de los sistemas de comunicaciones submarinas por medio de las tecnologías acústicas, radiofrecuencias y ópticas

Se observa que, las comunicaciones con radiofrecuencia proporcionan altas velocidades de datos, pero limitadas a una fuerte atenuación por la conductividad del agua de mar, las comunicaciones ópticas proporcionan velocidades de datos mucho más altas, 
sin embargo, están sujetas por la atenuación de la turbidez del agua de mar, las ondas acústicas proporcionan amplias distancias de trasmisión, pero presentan bajas tasas de trasmisión de datos y se encuentran sujetas a las reflexiones debido a la propagación multicamino. En la Figura 2.3, se evidencia el porqué de la selección de las ondas acústicas en comparación de las tecnologías descritas anteriormente.

Con todo lo anterior, el entorno marino desempeña un papel muy complejo en la transmisión de las señales. Este puede ser el principal problema encontrado en el uso de las comunicaciones submarinas. Por lo tanto, justifica los importantes esfuerzos realizados durante el último medio siglo en el estudio y modelado de la propagación acústica bajo el agua, y así tenerlos en cuenta en el diseño de los sistemas de comunicaciones de acústica submarina.

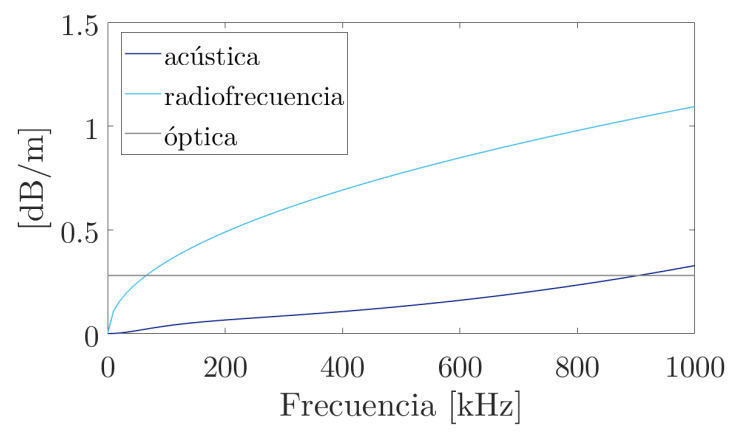

Figura 2.3: Comparativa de la atenuación en función de la frecuencia, exceptuando las comunicaciones ópticas

\subsection{Las comunicaciones acústicas submarinas}

\subsubsection{Revisión histórica}

La posibilidad de utilizar el sonido para detectar barcos distantes escuchando el ruido que irradian al agua, fue estudiado en 1490 por primera vez por Leonardo da Vinci [46]. Sin embargo, las aplicaciones prácticas son más recientes, y los primeros dispositivos de comunicaciones acústicas submarinas utilizados de manera eficiente fueron los sistemas de detección pasiva desarrollados durante la Primera Guerra Mundial. La idea de que los obstáculos a la navegación y los objetivos podrían ser detectados por los sistemas acústicos activos se había estudiado desde el comienzo del siglo XX. Este esfuerzo de investigación se vio estimulado, en particular, por la pérdida del Titanic en 1912. Paul Langevin, realizó experimentos en el mar y en el río Sena entre 1915 y 1918, y demostró que era posible transmitir señales y detectar submarinos usando sus 
ángulos y distancias desde el receptor. Su innovación consistió en usar un transductor piezoeléctrico, que trabajara a $38 \mathrm{kHz}$ [47]. Este concepto fue adoptado por la mayoría de las aplicaciones posteriores.

La tecnología del SONAR ${ }^{1}$ mejoró considerablemente entre las dos guerras mundiales y se benefició de la aparición de la electrónica. En 1941, EE. UU. invirtió en investigación y desarrollo de sonares, mejorando el rendimiento de los sistemas de sonares activos, así como la comprensión de la propagación de la onda acústica en el medio marino. Una gran parte del conocimiento del sonar básico que todavía se usa hoy, data de esta época.

Entre los primeros sistemas de comunicaciones acústicas, se tienen la comunicación de voz entre submarinos desarrollado por EE. UU. al final de la Segunda Guerra Mundial (1945); actualmente en uso. El sistema propuesto utilizaba una modulación analógica en amplitud con una banda frecuencial entre los 2 y los $15 \mathrm{kHz}$ [48]. Durante la Guerra Fría, también se desarrollaron varios sistemas basados en redes de comunicaciones inalámbricas submarinas, como por ejemplo, el sistema SOSUS (Sound SUrveillance System), que consistían en un conjunto de hidrófonos situados de manera estratégica en el fondo del mar para localizar submarinos soviéticos silenciosos [49].

Posteriormente en los años 70, con el desarrollo del (DSP ${ }^{2}$ ), se empiezan a implementar distintos tipos de modulaciones entre las cuales estan, la modulación de frecuencia combinada con la modulación de fase. Alrededor de la década de 1980, se alcanzan velocidades de transmisión de datos del orden de los kilobits por segundos (kbp) con las anteriores modulaciones [50]. En la década de los 90, se hace hincapié en la modulación de fase la cual alcanza velocidades de $16 \mathrm{kbps}$ con transmisiones a lo largo del eje de profundidades [51] y $10 \mathrm{kbps}$ para una transmisión en el eje de las distancias con separación entre emisor y receptor de $2 \mathrm{~km}$ [52]. Después, el Instituto Oceanográfico Woods Hole, desarrolla un sistema con velocidades de $40 \mathrm{kbps}$ para distancias de $2 \mathrm{~km}$ [53].

En los siguientes años, los avances consistieron en desarrollar otros tipos de modulaciones llamadas modulaciones avanzadas, en las que se encuentra, la técnica de modulación de espectro ensanchado por secuencia directa $\left(\mathrm{DSSS}^{3}\right)$ [54]. En el año 2004, se utiliza la técnica de modulación espacial y la $\left(\mathrm{OFDM}^{4}\right)[55,56]$.

Actualmente, se siguen empleando todas estas técnicas de modulación para los diferentes sistemas de comunicaciones en acústica submarina.

\footnotetext{
${ }^{1}$ Sound Navigation and Ranging

${ }^{2}$ Digital Signal Processor

${ }^{3}$ Direct Sequence Spread Spectrum

${ }^{4}$ Orthogonal Frequency Division Multiplexing
} 


\subsubsection{Aplicaciones}

En las comunicaciones acústicas submarinas, aparecen diversas aplicaciones en diferentes contextos, tales como: el científico, industrial/comercial y el de seguridad; como ejemplo se tiene la Figura 2.4. Las redes de sensores submarinos, sonares o ecosondas, permiten una forma de recopilar información del entorno marino para aplicaciones tales como:

- Control y monitorización de actividades comerciales como gasoductos y oleoductos. Con un sistema de comunicación, se podrían detectar posibles derrames o evitar fallos en la infraestructura e informarlo a la plataforma central de comunicaciones.

- Registro de datos ambientales del océano tales como, temperatura, salinidad, presión y niveles de oxígeno en el agua, que son importantes a corto plazo para la predicción de alteraciones naturales como maremotos y el seguimiento del cambio climático.

- Observación y estudio de la vida submarina, importante para mantener la biodiversidad, la fauna y la flora a través de la telemetría acústica.

- Control de la navegación y tránsito naval tanto en puertos como en zonas costeras o alta mar.

- Detectar y localizar obstáculos y objetivos; esta es la función primordial de los sistemas de sonar, principalmente para aplicaciones militares, como la lucha antisubmarina.

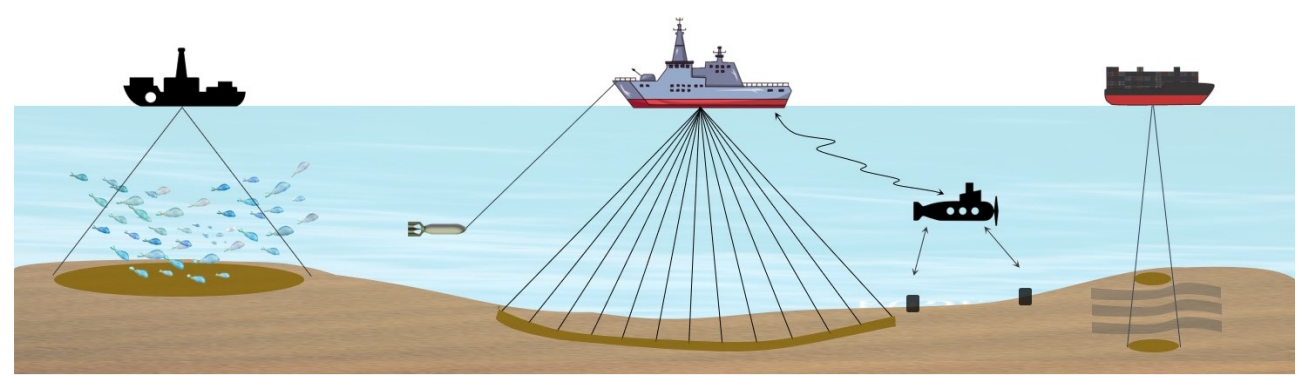

Figura 2.4: Ejemplos de aplicaciones para comunicaciones acústicas

Las anteriores aplicaciones, se logran a través de redes de sensores desplegadas en zonas marinas que se comunican por cable o de forma inalámbrica a través de nodos libres, comunicación entre submarinos, buques de superficie o sistemas operados remotamente, obteniendo así una red de comunicación heterogénea para transferir la información de los datos adquiridos al remitente inicial, además de informar de 
posibles peligros, como la detección de una intrusión o un evento peligroso para el ecosistema marino.

\subsubsection{Comunicaciones acústicas paramétricas}

El creciente interés en las comunicaciones acústicas, puede atribuirse a las necesidades científicas y comerciales, esenciales para la exploración de recursos marinos, el monitoreo del medio ambiente, defensa territorial, entre otros, que emplean, sistemas de comunicaciones tales como, $\left(\mathrm{AUV}^{5}\right)$ o $\left(\mathrm{UUV}^{6}\right)$ y/o Redes Inalámbricas de Sensores (RIS) o alámbricas [57-60].

Como se comentó en el apartado 2.1, el estudio de la propagación del canal acústico submarino, ha contribuido a mejorar los diseños en los sistemas de comunicación. Teniendo en cuenta que una de las limitaciones más relevantes en aguas poco profundas es la propagación multicamino, nace el estudio de los sistemas de comunicaciones acústicos paramétricos [61].

Las fuentes acústicas paramétricas, se empezaron a utilizar gradualmente en comunicaciones acústicas con sonar en aguas poco profundas, donde las reflexiones son mayores debido al multicamino [43-49], con el fin de obtener un mejor rendimiento, tal como lo presenta la Figura 2.5. Además de, trabajar en el diseño del ancho de banda de la señal de un array paramétrico para obtener altas velocidades de transmisión [43].
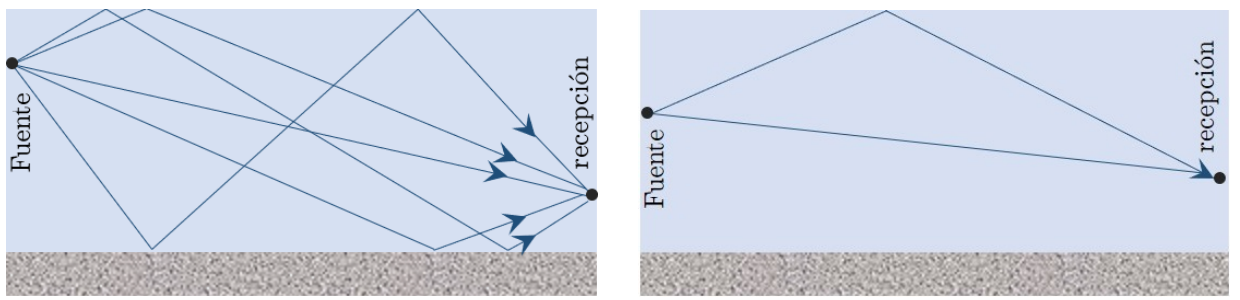

Figura 2.5: Ejemplo de las reflexiones debidas al multicamino en la propagación de la señal acústica en aguas poco profundas (izquierda) y mejora de estas debido al uso de fuentes paramétricas (derecha)

Aunque la teoría de la propagación de ondas no lineales en fluidos está muy bien estudiada y conocida desde el siglo XX, se han realizado pocos sistemas de comunicaciones paramétricos empleando la transmisión de datos digitales a través de las técnicas de modulaciones existentes. Un trabajo importante en este campo se realizó en la Universidad de Birmingham, en Reino Unido: BASS 300 PARACOM,

\footnotetext{
${ }^{5}$ Autonomous Underwater Vehicle

${ }^{6}$ Unmanned Underwater Vehicle
} 
cuyo proyecto de la Unión Europea $[62,63]$ obtuvo un enlace de comunicación paramétrico de largo alcance para su uso en telemetría. Emplearon un sistema de comunicación testeando diferentes modulaciones; FSK, MSK y BPSK, para posteriormente, obtener datos de comunicación con la modulación M-DPSK aplicando una frecuencia central de $300 \mathrm{kHz}$ y una frecuencia secundaria de $50 \mathrm{kHz}$. Los resultados muestran que, el sistema se puede emplear para combatir la propagación multicamino en aguas poco profundas. Este modelo se desarrolló, para confirmar las predicciones hacia un futuro enlace operacional de largo alcance, utilizando una frecuencia central de $50 \mathrm{kHz}$ y frecuencia secundaria de $5 \mathrm{kHz}$.

Las comunicaciones acústicas a través de la generación paramétrica, ofrecen grandes ventajas dado que, los haces de baja frecuencia generados por la modulación de una onda portadora, son altamente directivos, logrando así disminuir en su mayoría, las múltiples reflexiones generadas en aguas confinadas (poco profundas). Además, esta alta directividad en bajas frecuencias, se logra con el uso de transductores físicamente pequeños. Por lo tanto, se tendrían comunicaciones de largo alcance y baja frecuencia empleando una fuente compacta.

Con todo lo anterior, la desventaja de la transducción paramétrica, es su baja eficiencia de conversión $[62,64]$ que equivale por lo menos a una pérdida de potencia de $20 \mathrm{~dB}$. Además de esto, se requiere una alta potencia para el haz primario, alrededor de los cientos de Watios. Sin embargo, si no es necesario que el enlace de comunicación funcione todo el tiempo, como, por ejemplo, el caso de una aplicación petrolera que ofrece un gran suministro de energía, la transducción paramétrica puede ser una buena opción por emplear.

En la Tabla 2.2, se presentan los artículos en el ámbitos de las comunicaciones acústicas paramétricas, que emplean técnicas de modulaciones digitales, utilizadas para transmitir información de datos. Posteriormente, en el siguiente apartado, se estudian estas técnicas de modulaciones tanto de forma analógica como digital, para la transmisión de datos en sistemas de comunicaciones. 


\begin{tabular}{|c|c|c|c|c|c|c|c|c|c|c|c|c|}
\hline 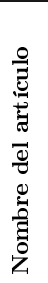 & 峦 & 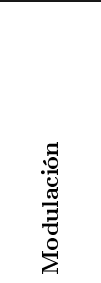 & 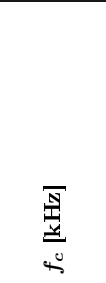 & 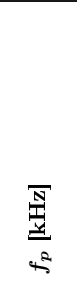 & 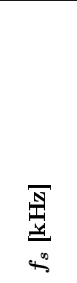 & 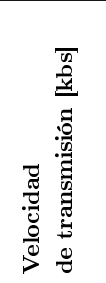 & $\begin{array}{c}\bar{N} \\
\frac{I}{D}\end{array}$ & 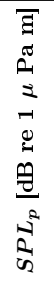 & 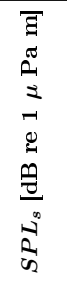 & 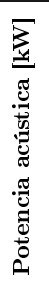 & 空 & $\frac{\bar{n}}{\frac{n}{2}}$ \\
\hline [62] & 1996 & DPSK & 300 & $\begin{array}{l}275 \mathrm{y} \\
325\end{array}$ & 50 & $\begin{array}{l}10 \mathrm{y} \\
20\end{array}$ & 10 & 228 & 201.9 & 2.2 & $10^{-5}$ & 30 \\
\hline [63] & 1996 & DPSK & 50 & $\begin{array}{l}47.5 \mathrm{y} \\
52.5\end{array}$ & 5 & 12 & 20 & & & & & 29.51 \\
\hline [65] & 1996 & FSK & 50 & $\begin{array}{l}47.5 \mathrm{y} \\
52.5\end{array}$ & $\begin{array}{l}2 \mathrm{y} \\
10\end{array}$ & $0.4 \quad 1.8$ & 10 & 231 & 190 & & $\begin{array}{l}4 \cdot 10^{-3} \mathrm{a} \\
6 \cdot 10^{-5}\end{array}$ & $\begin{array}{l}10 \mathrm{a} \\
30\end{array}$ \\
\hline [64] & 1996 & MDPSK & 50 & $\begin{array}{l}47.5 \mathrm{y} \\
52.5\end{array}$ & 5 & $1,2,3$ & 10 & 230 & 190 & 2.2 & $10^{-5}$ & 30 \\
\hline [66] & 2000 & DPSK & 50 & $\begin{array}{l}47.5 \mathrm{y} \\
52.5\end{array}$ & 5 & & & & & & $\begin{array}{l}0.56 \cdot 10^{-3} \mathrm{y} \\
1.9 \cdot 10^{-3}\end{array}$ & 30 \\
\hline [67] & 2008 & $\begin{array}{c}\text { LFM } \\
\text { BPSK } \\
\text { PAM } \\
\text { CPFSK }\end{array}$ & & & & & & & & & & \\
\hline [68] & 2012 & MIMO & & 560 & 45 & 0.013 & & & & 28 & $10^{-4}$ & \\
\hline [61] & 2012 & $\begin{array}{l}\text { PSK } \\
\text { QAM }\end{array}$ & & & & & & & & & & \\
\hline [69] & 2013 & $\begin{array}{l}\text { ASK } \\
\text { PSK } \\
\text { QAM }\end{array}$ & & & & & & & & & & \\
\hline [70] & 2015 & $\begin{array}{c}\text { LFM } \\
\text { PAM } \\
\text { BPSK }\end{array}$ & & & & & & & & & & \\
\hline [71] & 2016 & $\begin{array}{l}\text { BPSK } \\
\text { DSS }\end{array}$ & $\begin{array}{ll}153 & 157 \\
152 & 158 \\
151 & 159\end{array}$ & & $\begin{array}{ll}8 & 12 \\
7 & 13 \\
6 & 14\end{array}$ & $\begin{array}{l}0.031 \\
0.047 \\
0.062\end{array}$ & $\begin{array}{l}4 \\
6 \\
8\end{array}$ & & & & & \\
\hline [72] & 2016 & $\begin{array}{l}\text { PSK } \\
\text { QAM }\end{array}$ & & & & & & & & & & \\
\hline [73] & 2018 & Pulso & & & & & & & & & & \\
\hline [74] & 2019 & $\begin{array}{l}\text { QPSK } \\
\text { LFM } \\
\text { Pulso }\end{array}$ & 150 & & $\begin{array}{ll}8 & 12, \\
10 & \end{array}$ & & & & & & $6.67 \cdot 10^{-4}$ & 4.9 \\
\hline
\end{tabular}

Tabla 2.2: Artículos relevantes en el campo de las comunicaciones acústicas paramétricas

$f_{c}:$ frecuencia central

$f_{p}:$ frecuencia portadora

$f_{s}:$ frecuencia secundaria

$B$ : Ancho de banda
$S P L_{p}$ : Nivel de Presión Sonora de la portadora

$S P L_{s}$ : Nivel de Presión Sonora de la secundaria

$B E R$ : Bit error

$S N R$ : Relación señal ruido 


\subsection{Técnicas de modulaciones}

\section{Introducción}

La modulación es el conjunto de técnicas empleadas para transportar cierta información sobre una señal llamada portadora o central, que altera algunas de sus características (amplitud, frecuencia, o la fase) en función de otra señal, conocida como moduladora, dando lugar a una señal modulada. Las modulaciones son típicamente utilizadas en sistemas de comunicaciones con sonares lineales y paramétricos [75].

La Figura 2.6 muestra un esquema de un proceso de modulación genérico, válido para todo tipo de modulaciones, que incluye la nomenclatura que se empleará a lo largo de este capítulo.

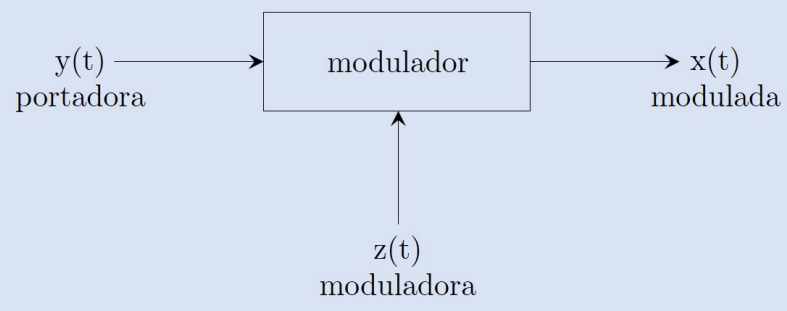

Figura 2.6: Esquema genérico de modulación

El esquema involucra las señales comentadas a continuación:

- $z(t)$ : señal moduladora, que contiene la información que se quiere transmitir.

- $y(t)$ : señal portadora, que ofrece el soporte para trasladar de frecuencia la señal moduladora; de tipo seno frecuentemente, es decir, $y(t)=A_{p} \omega_{p} \cdot t$, donde $\omega_{p}=$ $\sin \left(2 \pi f_{p}\right)$.

- $x(t)$ : señal modulada, generada del proceso de modulación, es decir, el producto de la portadora con la moduladora.

La señal moduladora puede ser analógica o digital, esta depende de la técnica de modulación que se emplee. Así, si se emplea la técnica de modulación analógica, la moduladora será $z(t)=\omega_{m} \cdot t$, donde $\omega_{m}=\sin \left(2 \pi f_{m}\right)$ y si, por el contrario, se utiliza una modulación digital, la moduladora consistirá en un conjunto de dígitos binarios conformados por el bit 1 y 0 . Seguidamente, se comentan las técnicas de modulación analógicas y digitales. 


\subsubsection{Modulaciones analógicas}

Entre los tipos de modulación analógicas más utilizadas se tienen las modulaciones en amplitud $\left(\mathrm{AM}^{7}\right)$ y las modulaciones angulares. De entre las angulares está la de Frecuencia Modulada $\left(\mathrm{FM}^{8}\right)$ y la Modulación de Fase $\left(\mathrm{PM}^{9}\right)$, tal como se observa en la Figura 2.7. En esta tesis sólo se introducirá la modulación AM, las angulares podrán profundizarse en $[76,77]$.

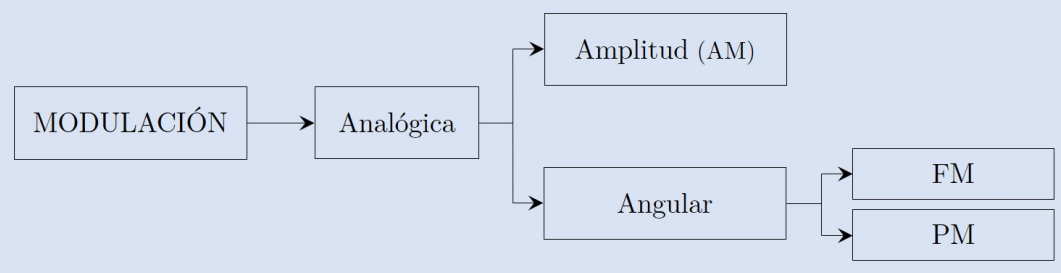

Figura 2.7: Tipos de modulación analógica

En cualquier tipo de modulación (analógica o digital), la señal portadora $y(t)$ tiene la siguiente forma [78].

$$
y(t)=A_{p} \sin \left(2 \pi f_{p} t+\varphi_{p}\right)
$$

Donde $A_{p}$, es el valor de la amplitud de la portadora, $f_{p}$, es la frecuencia de la señal portadora y $\varphi_{p}$, es la fase de la portadora. Cada una de estas variables están en función de la señal moduladora. Dependiendo de qué variable se aplica (amplitud, frecuencia o fase) se tiene la modulación en amplitud, modulación en frecuencia o en fase. A continuación, se analizan cada una de ellas.

\section{Modulación en amplitud (AM)}

La modulación en amplitud, es una técnica que consiste en cambiar la amplitud de la señal portadora $y(t)=A_{p} \sin \left(2 \pi f_{p} t\right)$, de frecuencia $f_{p}$ relativamente alta, en función de la amplitud de una señal moduladora tipo senoidal $z(t)=\sin \left(2 \pi f_{m} t\right)$, de frecuencia moduladora $f_{m}$ más baja. Representada como:

$$
x_{A M}(t)=y(t) \cdot z(t)
$$

\footnotetext{
${ }^{7}$ Amplitude Modulation

${ }^{8}$ Frequency Modulation

${ }^{9}$ Phase Modulation
} 
Donde $A_{p}$ es la amplitud de la portadora.

La Figura 2.8, muestra un ejemplo de una modulación AM, en la que se observa cómo la forma de onda de esta modulación llamada envolvente (figura azul) es idéntica a la señal moduladora, es decir, la envolvente de la señal modulada tiene la forma del mensaje.
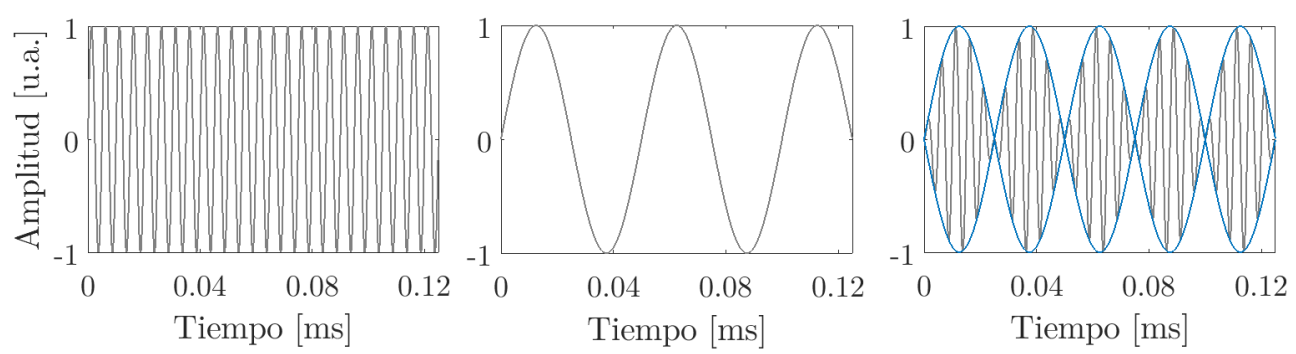

Figura 2.8: Ejemplo de una modulación en amplitud, representada por la onda portadora (izquierda), onda moduladora (centro) y señal modulada (derecha)

El resultado de la $\mathrm{AM}$ es desplazar la señal moduladora a una banda frecuencial alrededor de la portadora, de modo que el espectro frecuencial se corresponde a una banda lateral inferior $\left(f_{p}-f_{m}\right)$ junto a una banda lateral superior $\left(f_{p}+f_{m}\right)$. La Figura 2.9 presenta un esquema del espectro frecuencial para este tipo de modulación.

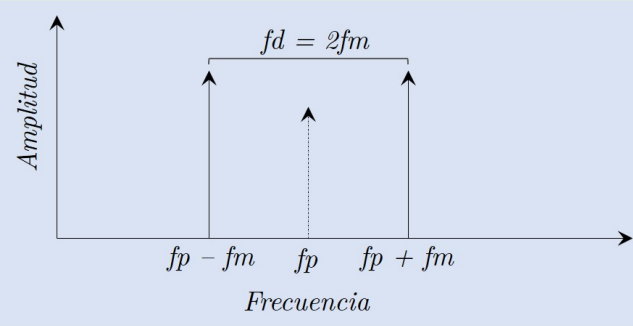

Figura 2.9: Esquema del espectro frecuencial de una modulación AM

\subsubsection{Modulaciones digitales}

Las modulaciones digitales permiten un uso más eficiente del ancho de banda del canal acústico submarino. Estas modulaciones, consisten en portadoras analógicas moduladas a través de una señal moduladora digital, donde el símbolo binario mas básico es el dígito binario o bit ( 1 o 0) [79]. Para expresar la velocidad de transmisión de estos dígitos, se emplean los bits por segundos (bps). Una de las ventajas de 
usar estas modulaciones, es que mejora la interferencia entre símbolos y tienen la posibilidad de implementar mecanismos que permitan la detección y corrección de posibles errores que suceden durante la transmisión. Su principal ventaja es que son inmunes al ruido. Estas señales se evalúan en un intervalo de muestras y se pueden guardar y procesar más fácilmente que las analógicas.

Modular en digital, consiste en convertir una señal digital en analógica donde variará su amplitud, frecuencia, fase o amplitud y fase conjuntamente, según los valores que vaya tomando la señal digital de información [79].

Partiendo de la expresión (2.1), Se tiene que, si la señal de información (moduladora) es digital, y la amplitud de la portadora varía en función de la moduladora se genera una modulación por commutación de amplitud $\left(\mathrm{ASK}^{10}\right)$. Si varía la frecuencia de forma proporcional a la moduladora, se tiene una modulación por commutación de frecuencia $\left(\mathrm{FSK}^{11}\right)$, si se varía la fase de la portadora en función de la moduladora, se tendrá una modulación por conmutación de fase $\left(\mathrm{PSK}^{12}\right)$. Por otro lado, si varía la fase y frecuencia se obtiene una modulación en cuadratura por conmutación de fase $\left(\mathrm{QPSK}^{13}\right) \mathrm{y}$, si lo que varía es la amplitud y fase de la portadora de forma proporcional a la señal moduladora, entonces se tendrá una modulación de amplitud en cuadratura $\left(\mathrm{QAM}^{14}\right)$.

En la siguiente Figura 2.10, se presenta un esquema de los diferentes tipos de modulaciones digitales que existen.

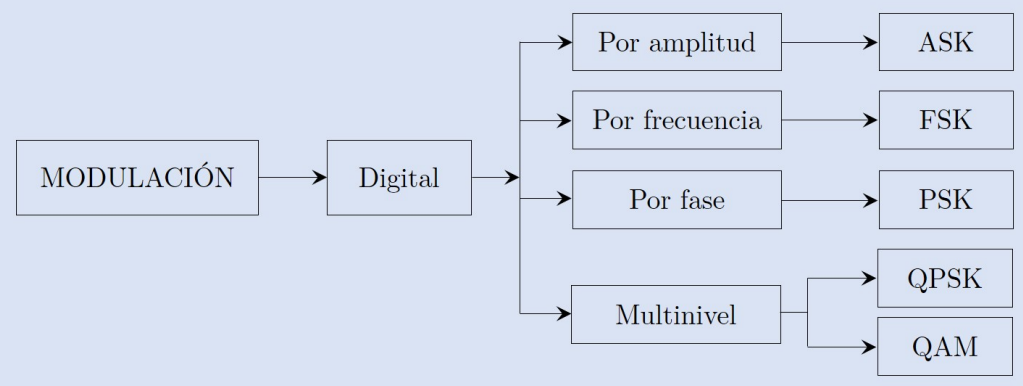

Figura 2.10: Tipos de modulación digital

\footnotetext{
${ }^{10}$ Amplitude Shift Keying

${ }^{11}$ Frequency Shift Keying

${ }^{12}$ Phase Shift Keying

${ }^{13}$ Quadrature Phase Shift Keying

${ }^{14}$ Quadrature Amplitude Modulation
} 
A continuación, se explican estas modulaciones digitales en su forma más básica, es decir, empleando símbolos sin codificarlos a un nivel mayor que el binario, y podrán profundizarse en $[78,80,81]$.

\section{Modulación por desplazamiento de amplitud (ASK)}

Esta modulación, representa los datos digitales como variaciones de voltaje de la onda portadora. La amplitud de una señal portadora analógica, varía conforme a la amplitud del bit 1 o 0 , manteniendo la frecuencia y la fase constante. Cuando la portadora equivale a un 1 lógico se dice que está encendida, y cuando equivale a 0 lógico está apagada. Esta forma de modulación ASK se denomina $\left(\mathrm{OOK}^{15}\right)$. A continuación, se presenta la expresión que define esta modulación a través de una señal binaria.

$$
x_{\mathrm{ASK}}(t)=\frac{1}{2}[1+z(t)]\left[A_{p} \sin \left(2 \pi f_{p} t\right)\right]
$$

La señal binaria moduladora $z(t)$ tiene amplitud $1 \mathrm{~V}$ y $-1 \mathrm{~V}$ para el bit 1 y 0 lógico respectivamente. Entonces, en función del bit a enviar, esta expresión toma la forma:

$$
\begin{aligned}
& x_{\text {OOK }[\mathrm{bit} 1]}(t)=A_{p} \sin (2 \pi f t) \\
& x_{\text {OOK }[\mathrm{bit} 0]}(t)=0
\end{aligned}
$$

En la Figura 2.11, se presenta un ejemplo para este tipo de modulación.
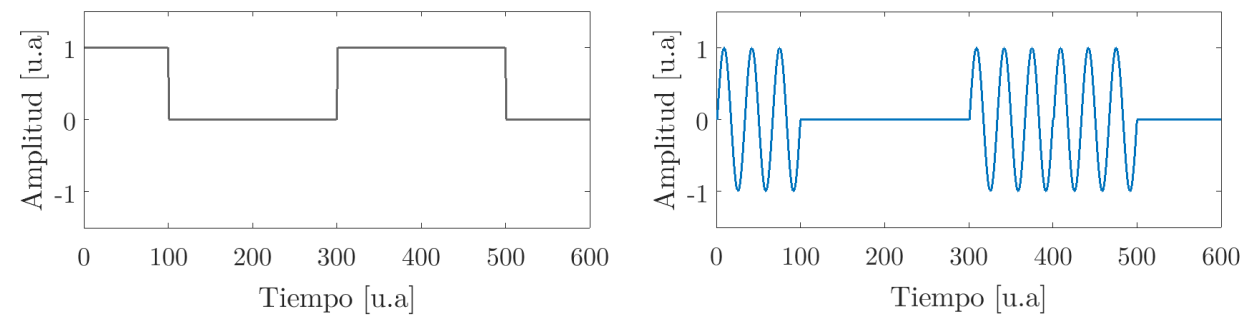

Figura 2.11: Ejemplo de una modulación OOK para un código binario [ $\left[\begin{array}{llllll}1 & 0 & 0 & 1 & 1 & 0\end{array}\right]$. Onda moduladora (izquierda) y señal modulada (derecha)

${ }^{15}$ On-Off Keying 


\section{Modulación por desplazamiento de frecuencia (FSK)}

Es una modulación de amplitud constante que utiliza una frecuencia portadora, la señal moduladora, es una señal binaria $z(t)$ que varía entre un 1 y 0 lógico, y cada uno se corresponde a una frecuencia determinada. Su representación matemática está dada como sigue:

$$
x_{\mathrm{FSK}}(t)=A_{p} \sin \left[2 \pi\left(f_{p}+z(t) \Delta f\right) t\right]
$$

La señal binaria moduladora $z(t)$ tiene amplitud $1 \mathrm{~V}$ y $-1 \mathrm{~V}$ para el bit 1 y 0 lógico respectivamente, y $\Delta f$ es la desviación máxima de frecuencia. En función del bit a enviar, esta expresión toma la siguiente forma:

$$
\begin{aligned}
& x_{\mathrm{FSK}[\mathrm{bit} 1]}(t)=A_{p} \sin \left[2 \pi\left(f_{p}+\Delta f\right) t\right] \\
& x_{\mathrm{FSK}[\mathrm{bit} 0]}(t)=A_{p} \sin \left[2 \pi\left(f_{p}-\Delta f\right) t\right]
\end{aligned}
$$

En la Figura 2.12 se presenta un ejemplo de la modulación FSK. Se observa que, cuando la señal moduladora binaria cambia de un 1 a un 0 lógico y viceversa, la señal modulada se desplaza entre dos frecuencias $f_{1}$ y $f_{2}$.
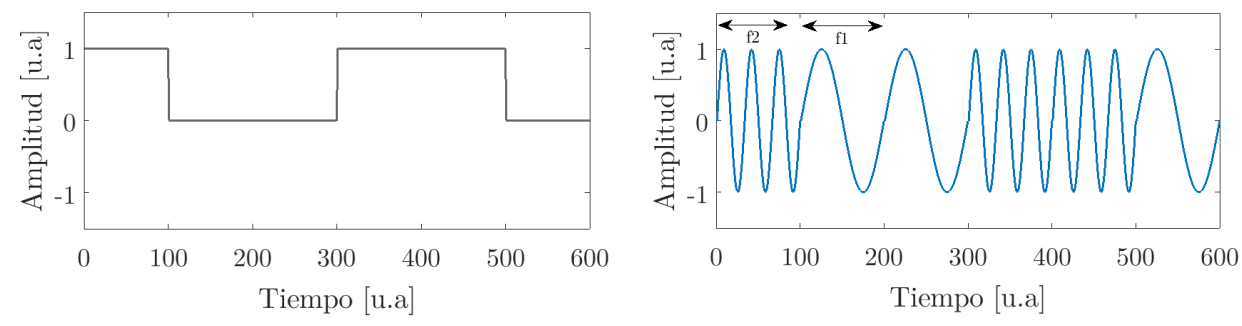

Figura 2.12: Ejemplo de modulación FSK para un código binario $\left[\begin{array}{llllll}1 & 0 & 0 & 1 & 1 & 0\end{array}\right]$. Onda moduladora (izquierda) y señal modulada con las dos frecuencias (derecha)

\section{Modulación por desplazamiento de fase (PSK)}

La modulación PSK binaria [57] $\left(\mathrm{BPSK}^{16}\right)$, consiste en variar la fase de la señal portadora según el símbolo de la moduladora digital $z(t)$ (1 o 0 lógico). Asignando $+1 V$ al 1 lógico y $-1 V$ al 0 lógico, y multiplicando la señal portadora por cada uno de esos valores, se obtienen las siguientes expresiones. Donde una fase representa un

\footnotetext{
${ }^{16}$ Binary Phase Shift Keying
} 
1 lógico $\left(0^{\circ}\right)$ y la otra un 0 lógico $\left(180^{\circ}\right)$. Al cambiar de estado la señal moduladora, la fase de la portadora varía entre esos dos ángulos.

$$
\begin{aligned}
x_{\mathrm{PSK}[\mathrm{bit} 1]}(t) & =A_{p} \sin \left(2 \pi f_{p} t\right) \cdot(1)=A_{p} \sin \left(2 \pi f_{p} t\right) \\
x_{\mathrm{PSK}[\mathrm{bit} 0]}(t) & =A_{p} \sin \left(2 \pi f_{p} t\right) \cdot(-1)=-A_{p} \sin \left(2 \pi f_{p} t\right)
\end{aligned}
$$

La expresión (2.9) indica que la señal se encuentra en fase, mientras que en (2.10), la señal está desfasada $180^{\circ}$. En el ejemplo de la Figura 2.13 se puede ver dicha modulación.
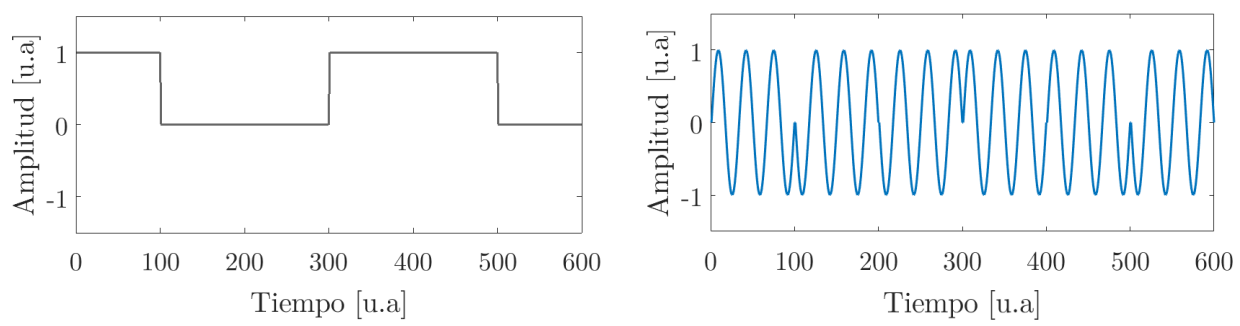

Figura 2.13: Ejemplo de modulción PSK para un código binario $\left[\begin{array}{llllll}1 & 0 & 0 & 1 & 1 & 0\end{array}\right]$. Onda moduladora (izquierda), señal modulada (derecha)

\section{Modulaciones Multinivel M-Aria}

Hasta ahora, se han comentado dos posibles combinaciones binarias, las modulaciones FSK y PSK, que codifican bits individuales y tienen dos condiciones lógicas de salida ( 1 o 0 ). Sin embargo, con las modulaciones multinivel, se pueden tener entradas digitales con más de dos niveles que representa la cantidad de combinaciones posibles para determinada cantidad de variables binarias, dado que a veces es necesario codificar a un nivel mayor $[77,78]$. Por ejemplo, QPSK que equivale a 4-PSK presenta 4 posibles fases de salida $M=4$ y así para, 8-PSK, 16-PSK. La condición de salida posible se calcula como:

$$
M=2^{N}
$$

Donde $N$ es la cantidad de bits codificados y $M$ es la cantidad de condiciones posibles de salida con dicho número de bits. Así, si entran dos bits codificados entre ellos, se tiene que las condiciones de salida equivalen a: $M=2^{2}=4$. 
De entre los tipos de modulaciones $M$-Aria, se tienen las modulaciones multinivel QPSK y sus variantes 8-PSK, 16-PSK. A continuación, se describe:

- Modulación en cuadratura (QPSK). Es una técnica de modulación angular que mantiene su amplitud constante. En esta modulación, cuando $M=4$, son posibles 4 fases en la salida de la modulación para una sola frecuencia portadora. Así, se deben tener cuatro condiciones distintas de entrada, como son: 00, 01, 10, 11.

Respecto a la modulación 8-PSK y 16-PSK, equivalen a $M=8$ y $M=16$ respectivamente. En la primera, se tiene que hay 8 posibles fases de salida, por lo que se organizan los bits en grupos de tres $2^{3}=8$ y en la segunda, existen 16 posibles fases de salida, por lo que los bits se organizan en grupos de cuatro bits $2^{4}=16$.

A continuación, se presenta su expresión matemática.

$$
x_{\mathrm{QPSK}}(t)=\left[\sin \left(2 \pi \frac{f_{b}}{N} t\right)\right]\left[\sin \left(2 \pi f_{p} t\right)\right]
$$

El primer término del lado derecho de la expresión, corresponde a la fase de la señal moduladora y el segundo término, corresponde a la fase de la señal portadora sin modular.
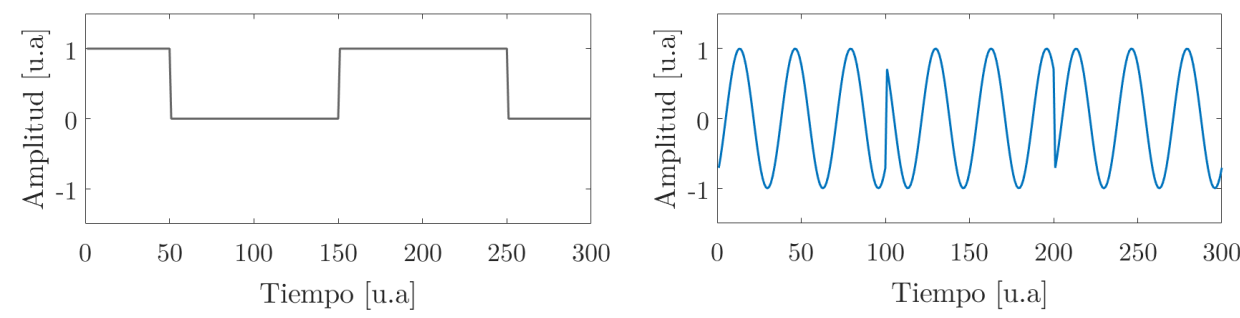

Figura 2.14: Ejemplo de una modulación QPSK para un código binario [ $\left[\begin{array}{llllll}1 & 0 & 0 & 1 & 1 & 0\end{array}\right]$. Señal moduladora (izquierda), señal modulada (derecha)

En general, los tipos de modulaciones M-Aria pretenden obtener una velocidad en bps mucho mayor para un canal con un ancho de banda específico, en comparación con las obtenidas de las modulaciones ASK, FSK y PSK para ese mismo canal.

De entre las modulaciones $M$-Aria, la que consigue una mayor inmunidad frente al ruido es aquella que presenta una $M$ menor. Esto se debe a que en una modulación QPSK se tiene una separación angular de $90^{\circ}$ y por lo tanto la señal presenta un 
desplazamiento de fase de $\pm 45^{\circ}$ sin perder información. En 8-QPSK se tiene una separación de $45^{\circ}$, por lo tanto, su margen de confiaza queda reducido a $\pm 22.5^{\circ}$. Para 16-QPSK la separación angular entre fases es de $\pm 22.5^{\circ}$ y su desplazamiento es de hasta $\pm 11.25^{\circ}$. Por esta causa, esta última modulación no se usa, dado que es mas suceptible a irregularidades $[77,79]$.

\subsection{Modulaciones para señales no lineales tipo paramétrico}

\subsubsection{Introducción a las modulaciones para comunicaciones no lineales}

El proceso de obtención de señales paramétricas para comunicaciones acústicas submarinas puede enfocarse desde dos puntos de vista: el proceso directo y el proceso inverso.

\section{Proceso directo e inverso}

Esta tesis se enfoca en el proceso directo, que corresponde a la aplicación directa del proceso de autodemodulación estudiado en el Capítulo 1, es decir, a partir de una señal de comunicación dada por cualquiera de las expresiones del apartado 2.3, predecir cuál sería el resultado de la propagación no lineal. Con ello, se puede comprobar la eficiencia de la señal paramétrica resultante para comunicar de forma directiva la señal original. Entonces, tal como se vió en el Capítulo 1, el perfil temporal de la señal acústica paramétrica (haz secundario) se obtiene mediante la segunda derivada de la envolvente $E$ al cuadrado de la señal enviada:

$$
x_{\text {param }} \backsim \frac{d^{2}}{d t^{2}} E^{2}(t)
$$

Aunque aquí no se aplica el proceso inverso, cabe resaltar solamente, que consiste en la inversa de la autodemodulación y, corresponde a aplicar la raíz cuadrada de la doble integral de la señal que se desea obtener paramétricamente. Esto puede profundizarse en [67]:

$$
E(t)=\sqrt{\iint x_{\text {param }} d t}
$$


Como se vio a lo largo del apartado 2.3, las técnicas de modulaciones lineales se basan, en su mayoría, en la concatenación de señales analógicas tipo seno. Por ello, se puede sintentizar la aplicación del proceso directo a este tipo de señales, tal como se verá. Adicionalmente, por extensión, se analizan también señales tipo sweeps (barridos en frecuencia de señales senoidales) que pueden utilizarse como elemento concatenante de una comunicación paramétrica.

Se estudia en primer lugar, los esquemas de modulaciones lineales estándar; es decir AM, y utilizando la teoría de Westervelt, se modela la transmisión paramétrica; donde la autodemodulación de una onda de amplitud finita, corresponde al campo acústico de la señal paramétrica (haz secundario) que es proporcional a la segunda derivada de la envolvente al cuadrado de la señal enviada (haz primario). Con esto, se muestran los resultados del proceso directo, para cada señal individual mediante análisis teórico.

Se estudia la modulación AM con doble banda lateral para la generación del efecto paramétrico tal como proponen algunos autores en $[82,83]$ y se presenta un estudio con señales tipo seno y sweep paramétrico. Estas modulaciones, son formadas por la combinación de una señal con frecuencia portadora $f_{p}$ correspondiente a la alta frecuencia y una señal con frecuencia moduladora $f_{m}$ que es la baja frecuencia. En la siguiente Figura 2.15, se presenta un esquema del espectro frecuencial de las señales moduladas, que corresponden a una modulación AM con un seno y sweep paramétrico.
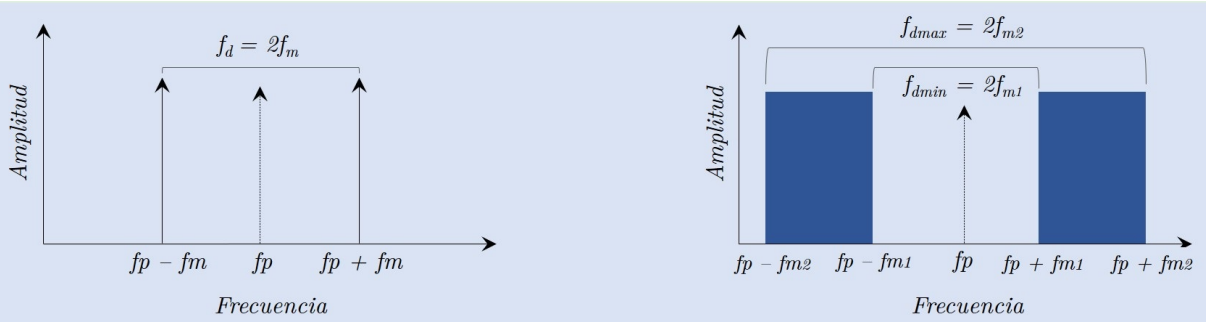

Figura 2.15: Esquema del espectro en frecuencia de una modulación AM para un seno paramétrico (izquierda) y para un sweep paramétrico (derecha)

La Figura 2.15 de la izquierda, representa una señal resultado de la modulación entre dos señales tipo seno, donde la propagación no lineal de esta señal, supondrá la aparición de la frecuencia diferencia $f_{d}$ que coincidirá con el doble de la frecuencia moduladora. La figura de la derecha, hace referencia al caso en que la moduladora sea una señal tipo sweep paramétrico, su propagación no lineal en el medio dará lugar a la generación de un barrido frecuencial cuya frecuencia diferencia inicial y final correspondan al doble de la frecuencia moduladora inicial $f_{m 1}$ hasta llegar al doble de la moduladora final $f_{m 2}$. 
Las señales generadas en esta tesis, son determinadas de acuerdo con la frecuencia que se desee producir paramétricamente en el medio, esto quiere decir que, partiendo de una frecuencia esperada en la recepción, se genera la correspondiente señal a emitir.

\section{Seno paramétrico}

El uso de este tipo de señales para estudios paramétricos viene dado por una parte, por su relación con las comunicaciones lineales y por otra, por su facilidad de generación originado mediante el efecto paramétrico. En consecuencia, su generación se basa en la obtención de una señal secundaria con frecuencia diferencia $f_{d}$ a partir de una señal primaria de dos frecuencias $f_{1}$ y $f_{2}$, de modo que $f_{d}=\left|f_{2}-f_{1}\right|$; o equivalentemente, una señal primaria modulada con frecuencia portadora $f_{p}$ y moduladora $f_{m}$, de modo que $f_{d}=2 f_{m}$.

La desventaja que presentan estas señales es una relativa mayor dificultad de detección al poseer un ancho de banda frecuencial estrecho, como se estudiará en el Capítulo 3, la correlación es más ancha y, por tanto, más difícil de discriminar respecto del ruido.

A continuación, se presenta la aplicación del proceso directo al caso del seno paramétrico.

Proceso directo: a partir de la señal senoidal conocida $z=\sin \left(2 \pi f_{m} t\right.$ ) (representa un bit de una modulación), que se utiliza como moduladora de una señal portadora $y=\sin \left(2 \pi f_{p} t\right)$, se obtiene la señal modulada $x=y \cdot z$, que corresponde a la señal primaria. Posteriormente, al aplicar la ecuación (2.13), se obtiene el perfil de la señal secundaria $x_{\text {param }}$, que toma la siguiente forma:

$$
x_{\text {param }} \backsim \frac{d^{2}}{d t^{2}} E^{2}(t)=\frac{d^{2}}{d t^{2}}|z|^{2}=8 \pi^{2} f_{m}^{2} \cdot \sin \left[2 \pi\left(2 f_{m}\right) t+\frac{\pi}{2}\right]
$$

Por lo tanto, el seno paramétrico es un tono de frecuencia, el doble de la moduladora $2 f_{m}$, desfasada $\pi / 2$ radianes y con una amplitud que depende de la frecuencia moduladora.

La Figura 2.16 muestra el efecto de aplicar este proceso: 

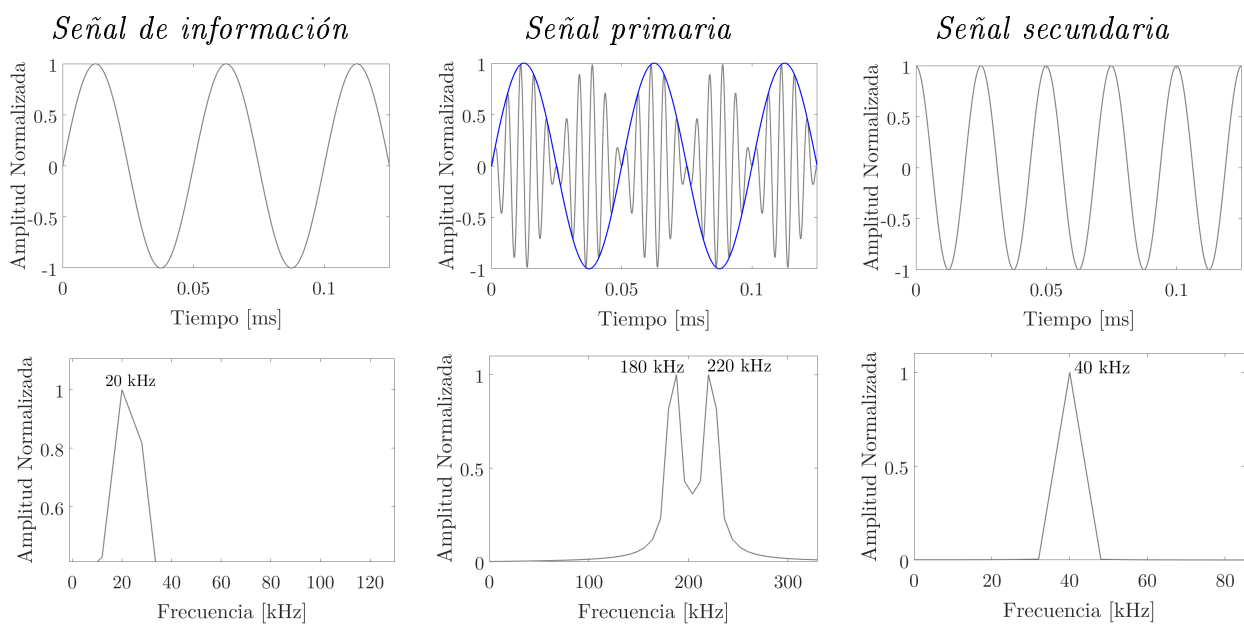

Figura 2.16: Proceso directo para un seno paramétrico en tiempo y frecuencia

\section{Sweep paramétrico}

Debido a que estas señales son barridos frecuenciales de señales tipo seno, tratan con un ancho de banda frecuencial mucho más amplio; por lo tanto, la ventaja que poseen es que su detección por el método de correlación cruzada se hace más precisa, debido a que se obtienen máximos bastante estrechos y definidos en comparación, con los senos paramétricos. Esto se estudiará en el siguiente Capítulo 3.

La modulación para un sweep paramétrico, se genera definiendo la señal moduladora en un intervalo de tiempo $\Delta t$, que corresponde a la duración $T$ de la señal y, otro intervalo frecuencial $\Delta f$ que se extiende desde $f_{m 1}$ hasta $f_{m 2}$ o viceversa, que son la frecuencia inicial y final del sweep respectivamente. En la Figura 2.17 se presenta el esquema que define esta modulación.

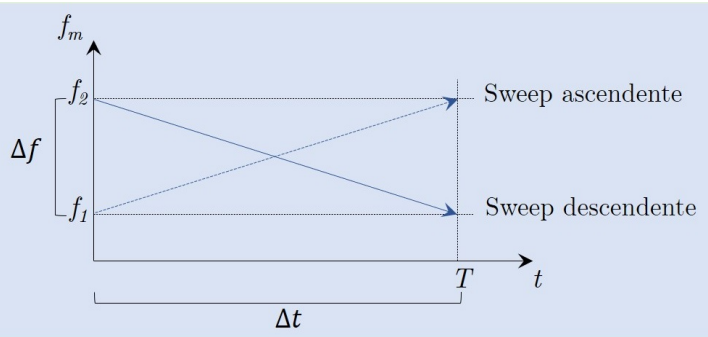

Figura 2.17: Esquema de la generación de una modulación tipo sweep paramétrico 
Proceso directo: a partir de una señal moduladora conocida que representa un bit $z=\sin \left(2 \pi f_{m} t\right)$; donde $f_{m}(t)=\frac{\Delta f}{\Delta t}+f_{1}$ con un frecuencia $f$ expresada en $H z$, y $t$ el instante de tiempo, expresado en segundos. Se tiene, el perfil de la señal secundaria $x_{\text {param }}$ expresado a continuación:

$$
\begin{aligned}
x_{\text {param }} \backsim \frac{d^{2}}{d t^{2}} z^{2} & =4 \pi \frac{\Delta f}{\Delta t} \sin \left[2 \pi \cdot 2 f_{m}(t) \cdot t\right] \\
+ & 8 \pi^{2}\left(\frac{\Delta f}{\Delta t} \cdot 2 t+f_{1}\right)\left(f_{m}(t)+\frac{\Delta f}{\Delta t} \cdot t\right) \cos \left[2 \pi \cdot 2 f_{m}(t) \cdot t\right]
\end{aligned}
$$

En esta ecuación, la señal secundaria esperada, es un sweep que corresponde al doble de la frecuencia moduladora y cuya amplitud crece proporcional a $\sim t^{3}$.

Al igual que se presentó un ejemplo gráfico del proceso directo analíticamente en la señal tipo seno paramétrico, se realiza el mismo estudio para la señal tipo sweep paramétrico. La Figura 2.18 muestra esquemáticamente, el efecto de aplicar este proceso. Se tiene la señal tipo sweep paramétrico desde los $4 \mathrm{kHz}$ hasta los $40 \mathrm{kHz}$ y con una duración de $1 \mathrm{~ms}$.

Señal de información
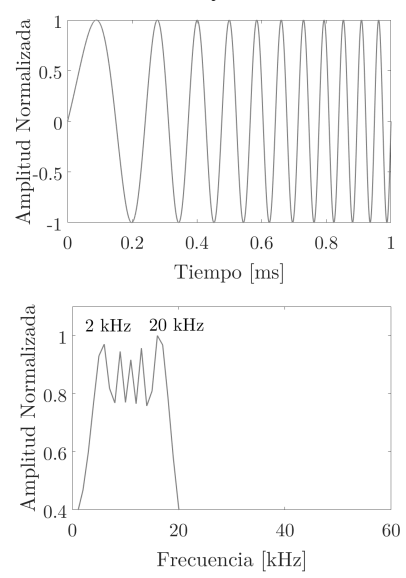

Señal primaria
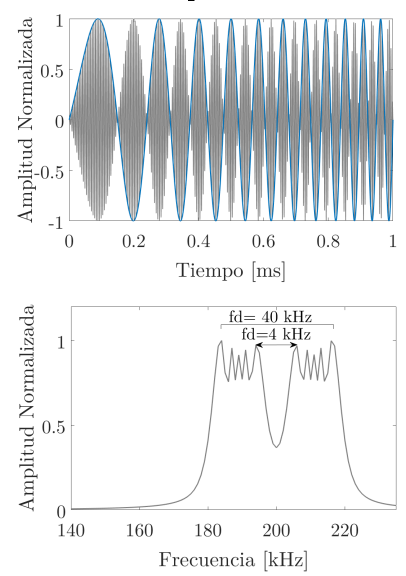

Señal secundaria
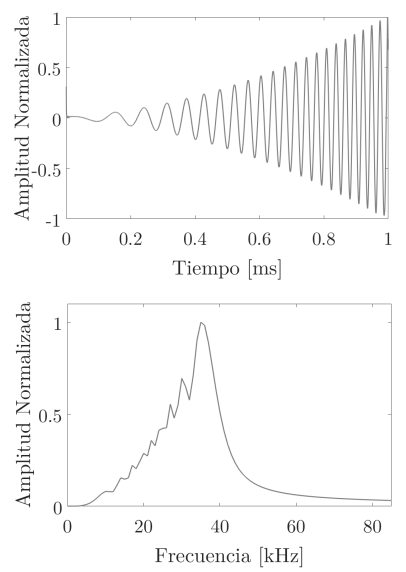

Figura 2.18: Proceso directo para un sweep paramétrico en tiempo y frecuencia 



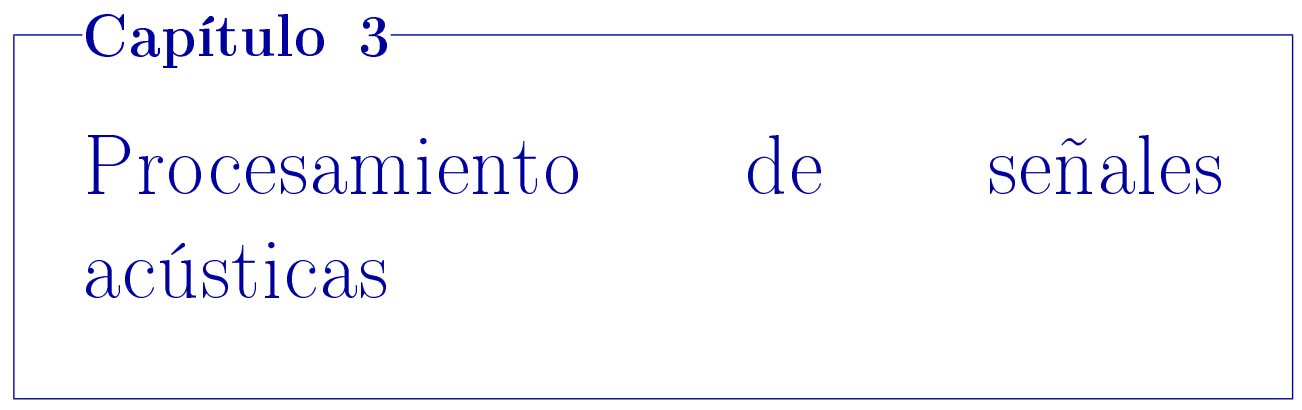

El procesamiento de señales acústicas consiste en la manipulación, transformación y representación de las señales, para obtener características o parámetros acorde al uso o aplicación que se desee estudiar, tales como: generación acústica, comunicaciones submarinas, calibración de transductores, entre otros. En esta tesis, todas las señales son digitalizadas de modo que las señales se discretizan en tiempo y amplitud dado que, de esta forma, la señal se puede representar mediante una secuencia de valores finitos que puede ser procesada a través de un computador. Consecuentemente, el tratamiento de señales empleadas se realiza en dicho contexto de procesado digital.

En en el apartado 3.1, se exponen los fundamentos de las técnicas empleadas para el procesado de señales en el dominio del tiempo, frecuencia y correlación. Al estudiar estos dominios, se obtienen diferentes técnicas para la parametrización de las señales acústicas que se aplican a las señales empleadas en esta tesis, cada cual más o menos adecuado según la aplicación.

En el apartado 3.2, se aborda la aplicación del procesamiento, a señales acústicas lineales utilizadas en la calibración de los transductores en emisión y recepción, y con ello, se describen los procesos inherentes para la caracterización (preprocesado, registro de las medidas y posprocesado).

En el apartado apartado 3.3, se ahonda en la aplicación al estudio de señales acústicas no lineales tipo paramétrico. Con todo, al trabajar de forma complementaria los distintos dominios, se obtienen las diferentes características comparables para las señales en régimen lineal y no lineal (paramétrico). 


\subsection{Técnicas para el procesado de señales}

En el diagrama de flujo de la Figura 3.1, se presenta el proceso por el que pasa la señal desde que se envía al sistema de generación, se propaga en el medio (agua) y finalmente, es recibida por el sistema de adquisición para, posteriormente, analizarla junto con la señal previamente enviada:

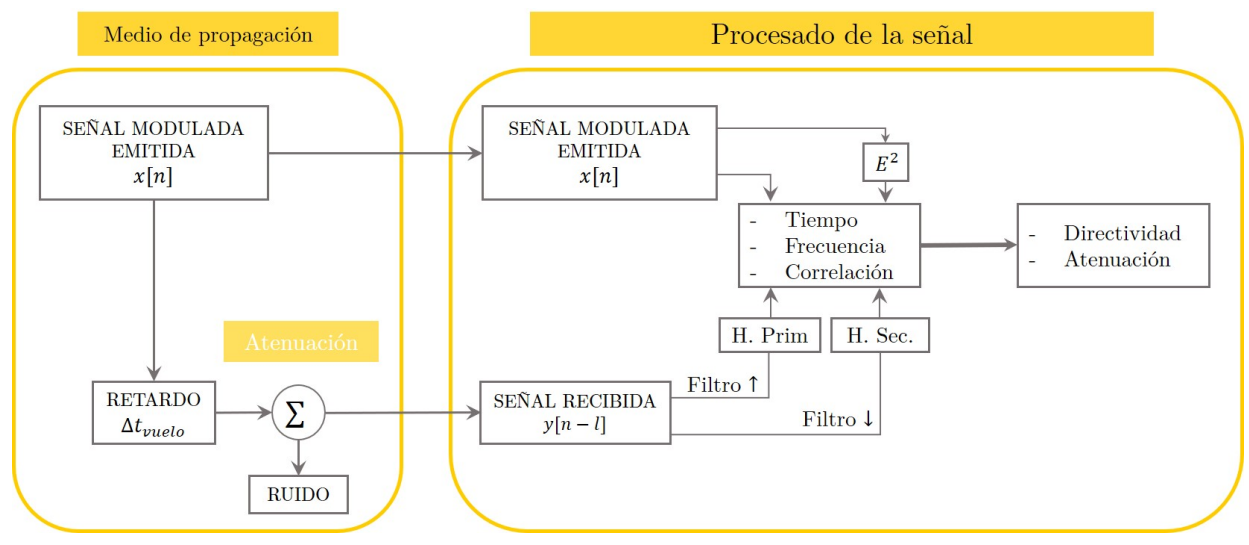

Figura 3.1: Diagrama de flujo de la señal enviada y recibida

Cuando la señal se propaga en el medio, sufre un retardo en su recepción debido al tiempo de vuelo. Además, llega parcialmente enmascarada por el ruido del sistema, y atenuada, debido a las pérdidas en la propagación. Por ello, para dilucidar las características de la señal bajo estudio, existen las técnicas de procesado. Cada una de ellas permite extraer unos parámetros o información relevante que se hallan ocultos o enmascarados en su representación original [84], y pueden ser utilizados para caracterizar o discriminar unas señales de otras.

Las técnicas empleadas en esta tesis se basan en un procesado en el dominio del tiempo, dominio de la frecuencia y de correlación. Cada una de estas técnicas se explica a continuación, remarcando la información que puede extraerse de la señal original.

Una vez la señal acústica se registra, se necesitan realizar ciertas técnicas de búsqueda o localización de eventos producidos en el momento en que la señal se grabó, ya que estas contienen un conjunto de información relevante que no pueden ser deducidas a simple vista. Esta búsqueda se conoce como detección, y es clave para poder identificar de forma precisa dichos eventos. 


\subsubsection{Procesado en el dominio del tiempo}

\section{El dominio del tiempo (transformada identidad)}

Describe el comportamiento de una señal respecto al tiempo. Es el dominio inherente de toda señal acústica, y a pesar de que es la representación más básica que se puede obtener empleando las herramientas, y en las condiciones de medida adecuadas, puede servir para extraer información relevante de la señal.

En el dominio temporal discreto, el valor de la señal o la función se conoce únicamente en algunos instantes del eje temporal, y sus amplitudes están discretizadas de modo que, no puede tomar cualquier valor, sino, únicamente aquellos dados por la profundidad de bits de su cuantificación. Así, las señales que se emplean son digitalizadas previamente a su procesado, y están formadas por una secuencia de $N$ muestras tomadas a intervalos regulares, separados por un periodo $T=1 / f_{s}$, siendo $f_{s}$, la frecuencia de muestreo. Ahora bien, el teorema de muestreo afirma, que para muestrear una señal de forma que se cumpla la condición de Nyquist $\left(f_{s}>2 f_{\max }\right)$, la frecuencia de muestreo deberá ser mayor que el doble de la frecuencia máxima de la señal. Con esto, será posible una reconstrucción satisfactoria de la misma.

En numerosos casos que son de interés en esta tesis, es útil conocer parámetros de la señal en determinadas frecuencias y/o bandas de frecuencias. Para obtener dicha información en el dominio del tiempo, la herramienta básica a emplear es el filtrado, proceso por el cual la parte útil o necesaria de una señal, se separa de otras componentes frecuenciales indeseadas. Por lo tanto, al filtrar la señal, se obtiene su perfil temporal para ese rango frecuencial, mostrando solo la amplitud resultante de ese ancho de banda deseado.

En el procesamiento digital de las señales acústicas analizadas en esta tesis, el filtrado se emplea para dos propósitos:

- Para eliminar ruidos indeseados presentes en las señales recibidas, es decir, las componentes frecuenciales que quedan fuera del rango de frecuencias de la señal a estudiar.

- Para estudiar la información de distintas bandas de frecuencia dentro del rango de frecuencias deseado. 


\section{Parametrización en tiempo}

Las señales en el dominio temporal, se pueden describir a través de parámetros de amplitud tales como, el valor de pico, de pico a pico, o rms. En esta tesis, para contrastar las amplitudes entre distintos dominios de análisis, se utiliza el valor de pico $V_{p}$ que, para señales armónicas estacionarias, se puede estimar como:

$$
V_{p}\{x[n]\}=V_{R M S}\{x[n]\} \cdot \sqrt{2}
$$

Donde $V_{R M S}$, es el valor $\mathrm{RMS}^{1}$ de la señal, definido como sigue a continuación.

$$
V_{R M S}\{x[n]\}=\sqrt{\frac{1}{N} \sum_{i=1}^{N} x_{i}^{2}[n]}
$$

Cuando se muestrea y recorta la señal, se puede obtener su amplitud en diferentes rangos frecuenciales (dentro de las frecuencias límite inferior $f_{\text {inf }}$ y superior $f_{\text {sup }}$ ), realizando distintos filtrados sobre ella. La Figura 3.2, muestra un ejemplo de valores pico obtenidos de una señal de banda frecuencial dada.
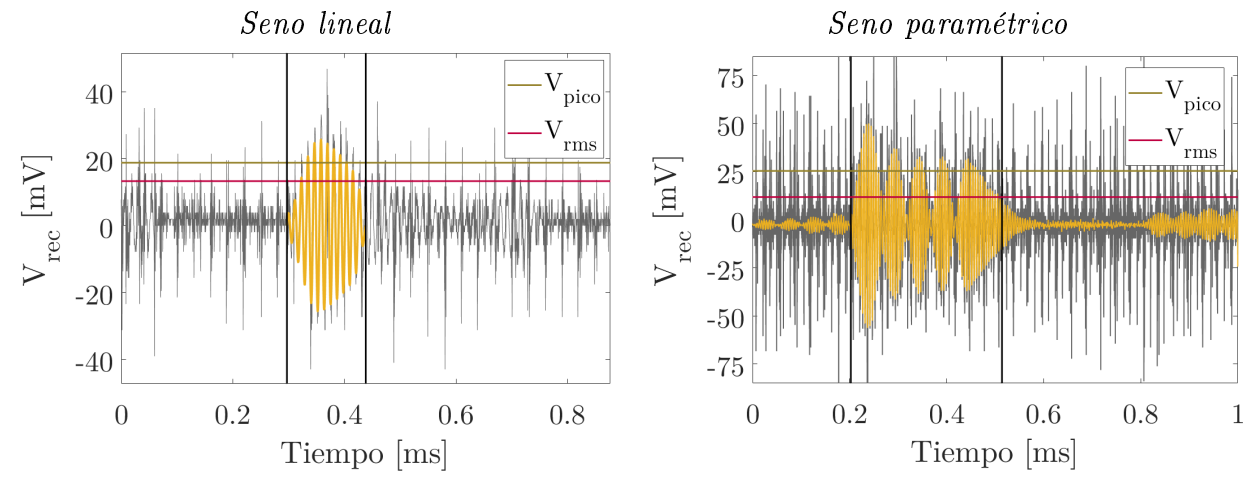

Figura 3.2: Ejemplo de una señal recibida en tiempo con ruido (gris) y señal recibida filtrada y enventanada entre $t_{1}$ y $t_{2}$ (ocre). Señal seno lineal (izquierda) y seno paramétrico (derecha)

Previo al filtrado de la señal, para extraer información en el dominio del tiempo, se toma la señal recibida y, se recorta en el tiempo que se cree estacionario, con el fin de, asegurar un buen comportamiento del filtro en el rango delimitado de tiempo deseado.

\footnotetext{
${ }^{1}$ Root Mean Square
} 


\subsubsection{Procesado en el dominio de la frecuencia}

\section{El dominio de la frecuencia (Transformada de Fourier)}

La Transformada de Fourier, transforma una señal que está representada en el dominio del tiempo al dominio de la frecuencia. Es una técnica ampliamente utilizada para representar las señales estacionarias, que ofrece características espectrales de una o varias frecuencias sin alterar su contenido.

En la Transformada de Fourier, la señal se interpreta como una superposición de ondas senoidales, de amplitud periódica, con frecuencia y fase constantes, es decir, de ondas estacionarias armónicas. La relación entre la señal original en el dominio de tiempo, $x(t)$, y la señal en el dominio de la frecuencia, $X(f)$, se relacionan a través de las siguientes expresiones [85,86].

$$
X(f)=\frac{1}{\sqrt{2 \pi}} \int_{-\infty}^{\infty} x(t) \mathrm{e}^{-j 2 \pi f t} d t \quad x(t)=\frac{1}{\sqrt{2 \pi}} \int_{-\infty}^{\infty} X(f) \mathrm{e}^{-j 2 \pi f t} d t
$$

En el caso de señales discretas, como las analizadas en esta tesis, la Transformada Discreta de Fourier, permite transformar un conjunto de valores obtenidos al muestrear la señal continua $x[n]$, al dominio discreto de la frecuencia $k$, de la siguiente forma [87].

$$
X[n]=\sum_{k=0}^{N-1} x[k] e^{\frac{-2 \pi k n}{N}}
$$

La mayoría de las señales bajo estudio, corresponden dentro de un intervalo de tiempo específico, a señales estacionarias (cuasi-estacionarias). Por ello, se estudia a continuación, los métodos adecuados de análisis (transformadas) y así poder obtener, aquellos parámetros que estan enmascarados en la señal original, tanto en el dominio del tiempo como en el de frecuencia.

\section{Parametrización en frecuencia}

Para analizar la señal en frecuencia, se debe aplicar un enventanado a la señal, es decir; recortarla previamente en el dominio temporal donde se encuentra la señal deseada (rango estacionario). Una vez realizado el proceso, se aplica la Transformada de Fourier a esta señal como se observa en la Figura 3.3 para, posteriormente, obtener 
el valor representativo de la amplitud de la señal a partir del pico resultante en la/las frecuencias de interés.

$$
V_{p, f r e c}=|T F\{V(t)\}| \frac{2}{N}
$$

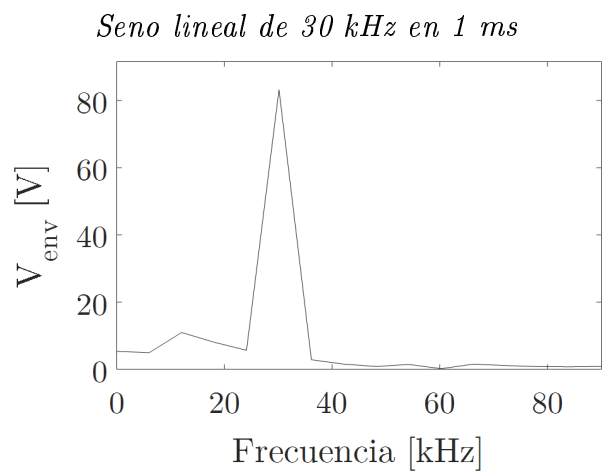

Seno paramétrico de $30 \mathrm{kHz}$ en $0.16 \mathrm{~ms}$

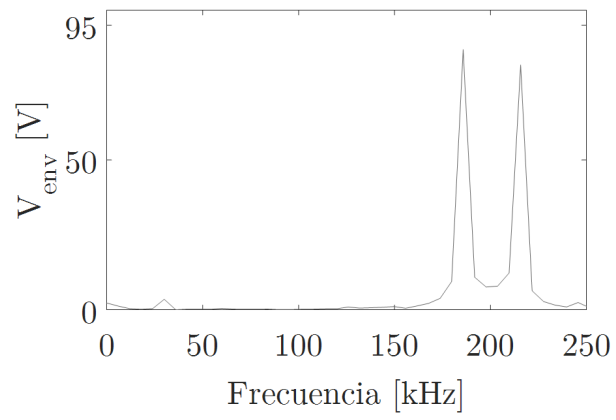

Figura 3.3: Representación espectral de una señal tipo seno lineal (izquierda) y seno paramétrico (derecha), ambas con frecuencia portadora de $200 \mathrm{kHz}$

Entonces, al recortar y realizar la Transformada de Fourier de esa ventana temporal de la señal, se perderá la distribución temporal de la señal completa, pero se habrá ganado en distribución frecuencial de la señal.

Concluyendo lo anterior, cuando se desea extraer información en el dominio de la frecuencia, se realizan los siguientes pasos:

- Se toma la señal recibida en el tiempo y se recorta temporalmente entre $t_{1}$ y $t_{2}$.

- Se obtiene la Transformada Fourier.

\subsubsection{Procesado en el dominio de la correlación}

\section{La correlación cruzada}

La correlación, es una operación matemática que se utiliza para medir el grado de semejanza entre dos señales [88]; esto es, si existe cierto parecido en las formas de ondas $x(t)$ y $y(t)$, empleadas para, extraer información relevante de una señal desconocida a través de la comparación con otra señal que sí se conoce. Se denomina autocorrelación, cuando se quiere determinar el parecido de una señal $x[n]$ consigo misma, y correlación 
cruzada, cuando se desea determinar el parecido entre dos formas de onda diferentes. Dado que todas las medidas se registraron mediante un sistema de adquisición digital, las señales en estudio se trabajan en tiempo discreto; con ello, la correlación entre las dos señales $x[n]$ y $y[n]$ con un número de muestras $N$, se representa mediante la siguiente expresión.

$$
r_{x y}[l]=\sum_{i=1}^{N} x[n] y[n+l]
$$

A efecto de aplicación en esta tesis, se supone que la señal digitalizada por el transductor emisor es $x[n]$, y la señal digitalizada del hidrófono es $y[n]$, donde $l=1,2, \ldots, N$, son el número de muestras en que $y$ se retrasa con respecto a $x$. Así, la función de correlación cruzada se determina para un número de valores $l$, y la estimación del tiempo de retardo estará entre los valores de $l$ en los que la función de correlación es máxima.

Dado que los fenómenos acústicos que se tratarán conllevan, en muchas ocasiones, una componente de señal directa y otra reflejada pudiendo llegar a haber solape entre ambas señales, el procesado en el dominio de la correlación ofrece una ventaja significativa que supone, la diferenciación entre dicha señal directa y la primera reflexión, así como la obtención de la amplitud de las frecuencias que contiene ambas señales.

\section{Parametrización en la correlación}

El método de la correlación cruzada sólo permite localizar el inicio de la señal en el tiempo, pero no se puede conocer directamente su valor de amplitud en unidades originales tales como, voltios o pascales. Sin embargo, existen estudios que permiten obtener dicho valor, a partir del pico de la correlación cruzada entre la señal recibida y la señal con la que se quiere comparar. Si se conoce la amplitud de una señal enviada, $V_{p, e n v}$, su número de muestras $N_{e n v}$, y el valor del máximo de la correlación entre la señal recibida y enviada, $V_{\max , c o r r}$ (situado en el instante de detección de la señal), es posible obtener el voltaje de amplitud pico de la señal recibida aplicando la siguiente expresión [89]:

$$
V_{p}\left[y_{r e c}\right]=\frac{2 V_{\text {max }, c o r r}}{V_{p, e n v} \cdot N_{e n v}}
$$

El pico máximo obtenido al correlar la señal recibida con la señal emitida, establece el tiempo de llegada de la señal recibida, tal como se observa en la Figura 3.4 que, para 
la señal recibida se tiene un tiempo de llegada de $0.25 \mathrm{~ms}$. Conociendo la velocidad de propagación del sonido en el medio $(\sim 1480 \mathrm{~m} / \mathrm{s})$ donde se transmite la señal, se determina la distancia que existe entre la emisión y la recepción. La velocidad de propagación del sonido en el agua varía ligeramente de una condición a otra dependiendo principalmente de la salinidad, la temperatura y la presión.

Seno paramétrico de $20 \mathrm{kHz}$ a $167 \mu \mathrm{s}$

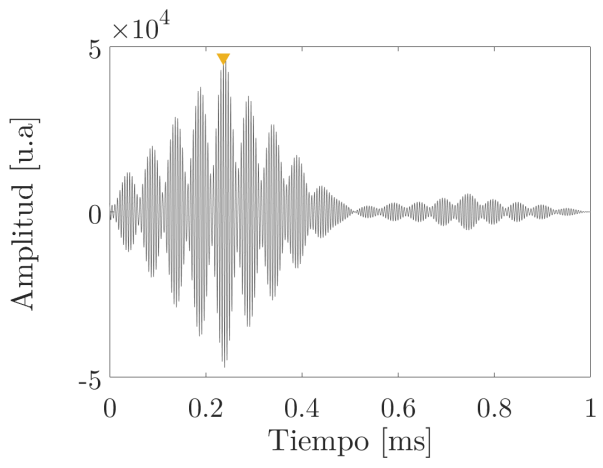

Sweep paramétrico de 4-40 kHz a $1000 \mu \mathrm{s}$

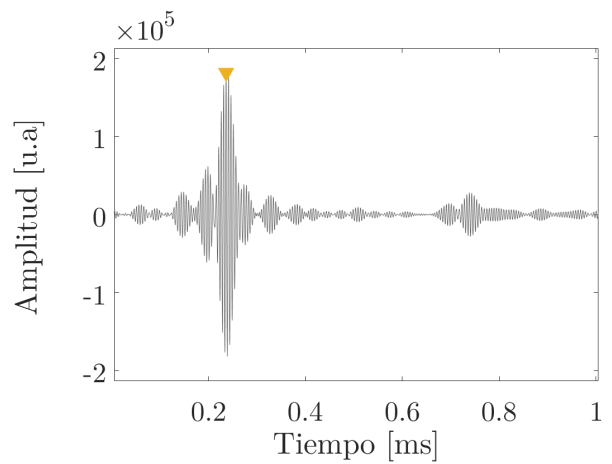

Figura 3.4: Representación de la correlación cruzada de un seno paramétrico (izquierda) y sweep paramétrico (derecha), ambos con frecuencia portadora de $200 \mathrm{kHz}$

Con todo, a diferencia de la representación en el dominio del tiempo y frecuencia, donde la información parte de un recorte de la señal. En la correlación, las amplitudes de la señal resultante indican cuánto se parecen dos señales (la señal con la que se trabaja respecto a una de referencia con la que se correla), dando lugar a una representación en el espacio del tiempo en el que la amplitud de esos picos, ofrece una identificación o un paramétro de la señal, que muestra donde se producen esos picos respecto al origen del tiempo de la señal enviada.

\subsection{Aplicación de las técnicas a señales lineales o de calibración}

Las técnicas de análisis descritas anteriormente se aplican en este apartado para caracterizar un transductor acústico y obtener su sensibilidad en emisión TVR, parámetro que será detallado en el Capítulo 4. A continuación, se describen los procesos llevados a cabo para obtener dichos resultados. 


\subsubsection{Procesos para la caracterización}

Los parámetros de interés para el preprocesado de la señal acústica y su consecuente obtención de la sensibilidad en emisión TVR, son los siguientes:

$\begin{array}{ll}f_{s} & \text { frecuencia de muestreo } 2 \mathrm{MHz} \\ f & \text { rango frecuencial estudiado (desde los } 10 \mathrm{kHz} \text { hasta los } 250 \mathrm{kHz}) \\ t_{e n v} & \text { duración de la señal enviada } \\ c_{m e d}, R V R & \text { propiedades del medio acústico (velocidad y sensibilidad) } \\ d_{E M} & \text { distancia emisor - receptor } 40 \mathrm{~cm}\end{array}$

Los datos o valores obtenidos en el análisis de la sensibilidad en emisión TVR son:

$V_{\text {env }} \quad$ señales enviadas por el transductor emisor [V]

$V_{\text {rec }} \quad$ señales recibidas por el hidrófono [V]

En la Figura 3.5, se presenta un esquema del proceso realizado para las medidas en cada frecuencia estudiada. En él, se observa en primera instancia, la señal enviada al sistema de adquisición. Se envían el rango de frecuencias $f$, descrito anteriormente con un tiempo $t$ que corresponde a 5 ciclos por cada señal. Una vez se reciben las señales para cada frecuencia, se realiza la autocorrelación de la señal enviada $V_{p, e n v, c o r r}, \mathrm{y}$ la correlación cruzada (XCorr) entre la señal recibida con la enviada para cada una de las frecuencias $V_{p, r e c, c o r r}$. Además de esto, se realiza el análisis en el dominio del tiempo y frecuencia. Se enventana la señal enviada y recibida a la duración de cada una, para analizar el contenido espectral con la Transformada de Fourier $V_{p, e n v, \text { frec }}$, $V_{p, r e c, f r e c}$. Respecto al dominio del tiempo, se filtra la señal enviada y recibida que ha sido previamente enventanada $V_{p, \text { env,tiempo }}, V_{p, \text { rec,tiempo }}$.

Una vez explicado el proceso anterior para la obtención de la caracterización en lineal de un transductor acústico piezoeléctrico, se realiza el análisis de las señales enviadas y recibidas en el siguiente subapartado.

\subsubsection{Resultados de caracterizar un transductor}

\section{Análisis de señales}

En este análisis, se parte de la señal enviada y recibida en el dominio del tiempo: la señal enviada contiene el perfil temporal de la señal senoidal de duración limitada, y en la señal recibida se tiene la señal registrada por el transductor con la recepción e influencia del ruido ambiente y reflexiones. A continuación, se muestra un ejemplo 


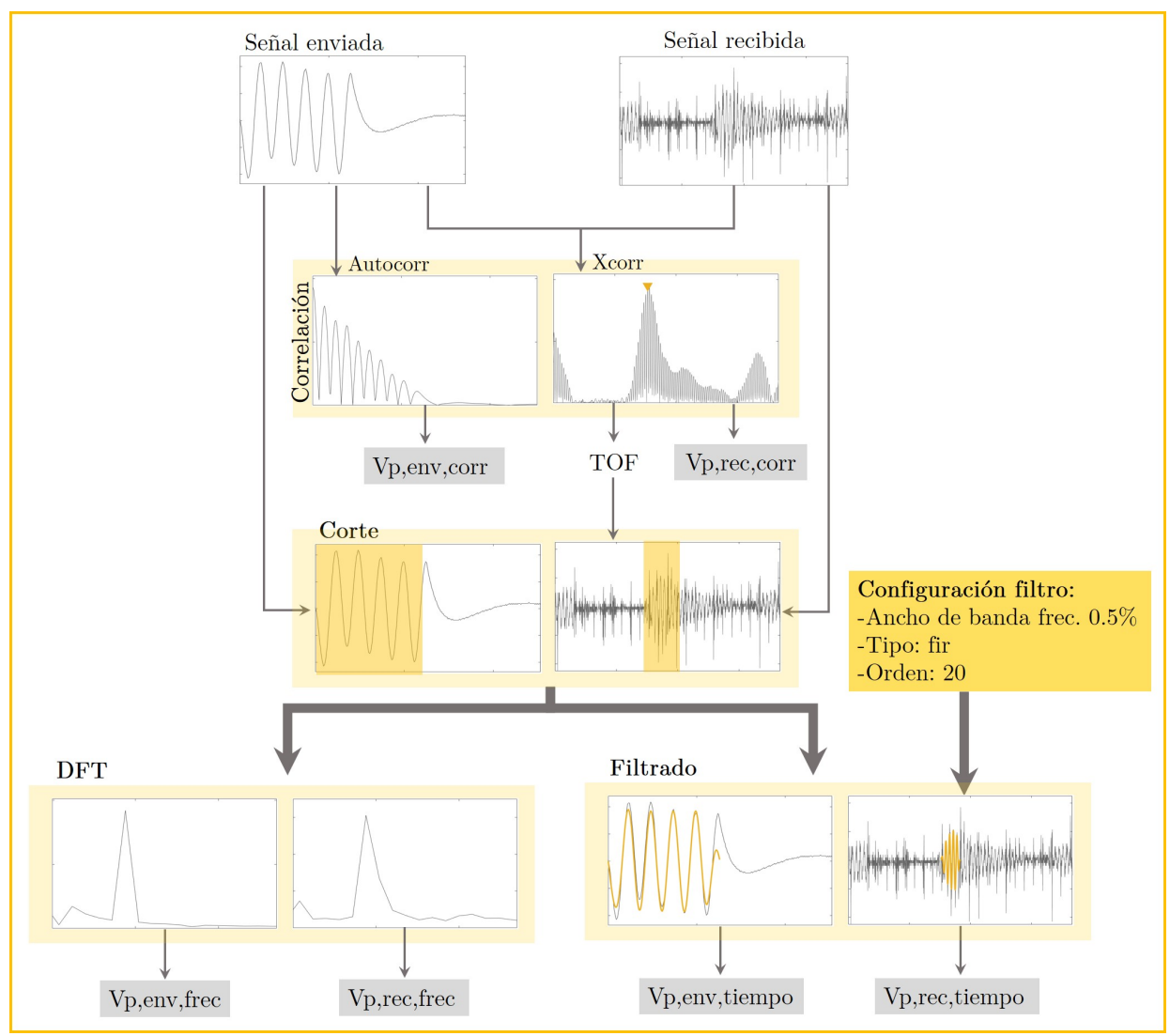

Figura 3.5: Esquema del procesado de la señal acústica en régimen lineal para caracterizar un transductor en función de su sensbilidad.

(seno de $88 \mathrm{kHz}$ ) del resultado obtenido al aplicar cada una de las transformaciones anteriores y las amplitudes resultantes en cada una de ellas. Posteriormente, se realiza una comparación de las amplitudes en estos tres dominios.

- En el dominio del tiempo, el tiempo de llegada está determinado por la diferencia entre el tiempo de emisión de la señal enviada previo filtro pasa banda, centrado en la frecuencia de la señal emitida como se observa a la izquierda de la Figura 3.6, y el tiempo inicial de la señal recibida. Con una distancia entre transductor emisor-receptor de $0.45 \mathrm{~m}$ medidos en piscina, el tiempo inicial de llegada de la señal recibida se espera en $0.3 \mathrm{~ms}$, tal como se presenta en la gráfica de la derecha. 

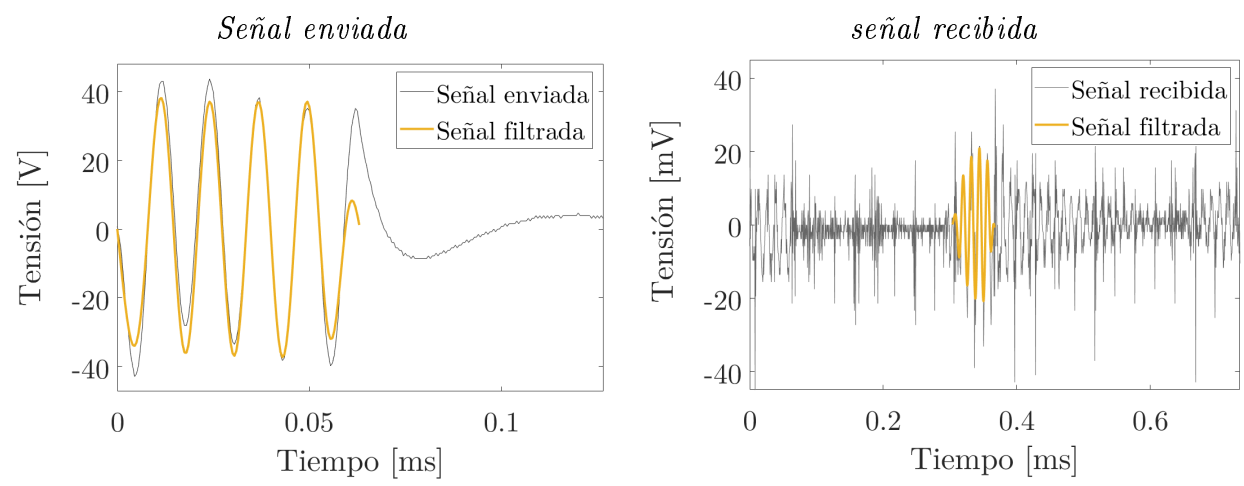

Figura 3.6: Técnicas de detección empleadas en el análisis de señales en el dominio del tiempo

- En el dominio de la frecuencia, como se comentó en el subapartado 3.1.2, una vez recortada la señal a analizar en tiempo, se aplica la FFT a esta señal, y se tiene el valor de amplitud del pico. En la Figura 3.7, se observa el valor de amplitud de pico, tanto para la señal enviada correspondiente a $38 \mathrm{~V}$ como para la recibida de $15 \mathrm{~V}$.
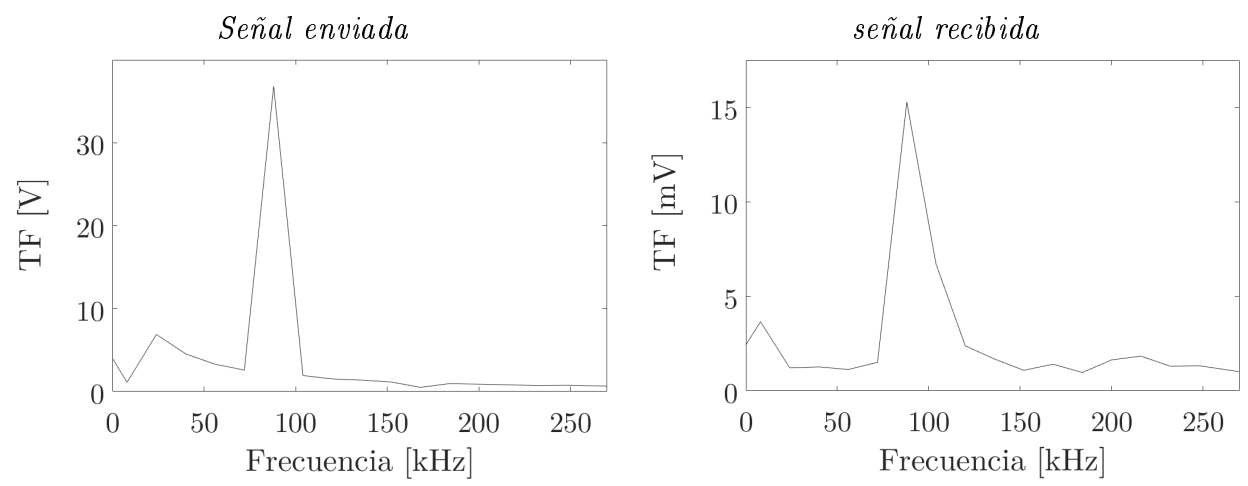

Figura 3.7: Técnicas de detección empleadas en el análisis de señales en el dominio de la frecuencia

- En el dominio de la correlación para la frecuencia de $88 \mathrm{kHz}$, la Figura 3.8 (izquierda), muestra la autocorrelación de la señal enviada, y se detecta un pico máximo en el origen. Respecto a la señal recibida, se tiene que, con el método de la correlación cruzada, se puede determinar el tiempo de llegada de esta, tomando el intervalo de tiempo que corresponde al pico máximo de la señal correlada, que 
para la gráfica de la derecha corresponde a $3 \mathrm{~ms}$. Esta técnica es más favorable para las señales de banda ancha (sweeps) porque tienen un máximo de correlación más estrecho y, en consecuencia, el pico medio es más fácil de distinguir de los otros picos con una buena precisión. Esto se verá en los siguientes apartados cuando se analicen senos y sweeps paramétricos.
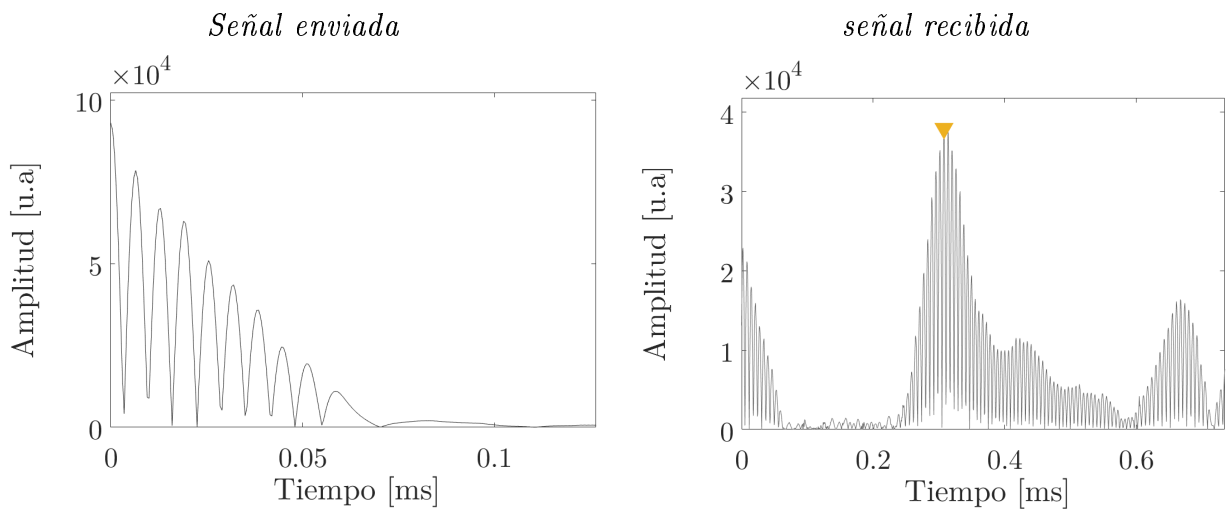

Figura 3.8: Técnicas de detección empleadas en el análisis de señales en el dominio de la correlación

En la Figura 3.9, se comparan las amplitudes de las señales recibidas que se emplearon en la calibración del transductor para los dominios estudiados anteriormente. Se tiene que, los valores obtenidos se aproximan entre sí, exceptuando una disminución en la amplitud en el dominio de la frecuencia a partir de $212 \mathrm{kHz}$. Las amplitudes en el dominio de la correlación presentan un ligero aumento en los picos máximos de 85 kHz y $200 \mathrm{kHz}$ en comparación con los otros dominios. En general se tienen valores de amplitud similares en los tres dominios.

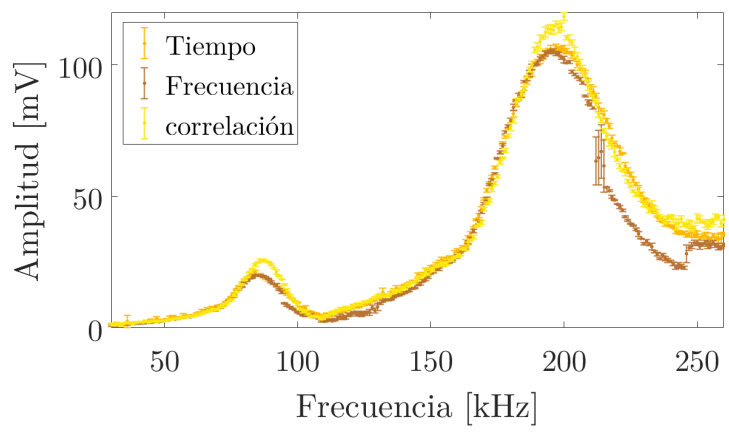

Figura 3.9: Comparación de las amplitudes en el dominio de tiempo, frecuencia y correlación 


\subsection{Aplicación de las técnicas a señales no lineales de comunicación}

En este apartado, se presenta la aplicación de las técnicas anteriores para el estudio de señales paramétricas utilizadas en las comunicaciones acústicas paramétricas submarinas. Como en este ámbito se trabaja con señales primarias y secundarias de alta y baja frecuencia, respectivamente, se mostrará la aplicación a cada una de ellas. Todo esto, con el fin de poder caracterizar el campo acústico no lineal (efecto paramétrico) generado por un transductor acústico piezoeléctrico en sus magnitudes de: directividad, variación de voltaje y atenuación, que serán estudiadas detalladamente en el siguiente Capítulo 4. A continuación, se presentan dichos procesos para la caracterización.

\subsubsection{Procesos para la caracterización}

Los parámetros de interés para el preprocesado de la señal acústica y la obtención de las diferentes caracterizaciones en régimen no lineal son:

$f_{s} \quad$ frecuencia de muestreo

$f \quad$ rango frecuencial estudiado (desde los $2 \mathrm{kHz}$ hasta los $200 \mathrm{kHz}$ )

$t_{\text {env }}$ duración de la señal enviada

$c_{\text {med }}$ propiedades del medio acústico (velocidad)

$d_{E M}$ distancia emisor - receptor

V voltaje

Los datos o valores obtenidos en los distintos análisis son los siguientes:

$V(t)_{e n v} \quad$ señales enviadas por el transductor $V_{p}$

$V(t)_{\text {env }} \quad$ señales recibidas por el hidrófono $V_{p}$

El esquema del proceso para la caracterización en no lineal presentado en la Figura 3.10, sigue un patrón similar al de la anterior Figura 3.5. En este sentido, se pretende analizar la señal paramétrica (baja frecuencia), es por esto que, de la señal enviada se extrae su segunda derivada de la envolvente al cuadrado y con esta, se realiza la correlación cruzada (XCorr) con la señal recibida. Claramente, se observa un pico de correlación en el tiempo en el que se espera la señal, llamado tiempo de vuelo (TOF) ${ }^{2}$. Además, se sigue el análisis en los dominios del tiempo y frecuencia. En el dominio de la frecuencia, se enventana la señal enviada y recibida, realizando

\footnotetext{
${ }^{2}$ Time of Flight
} 
un corte entre el tiempo inicial y final de la señal, y se analiza su espectro a través de la Transforma de Fourier. Para analizar la baja frecuencia, se filtra la señal recibida alrededor de la frecuencia de interés (señal en ocre).

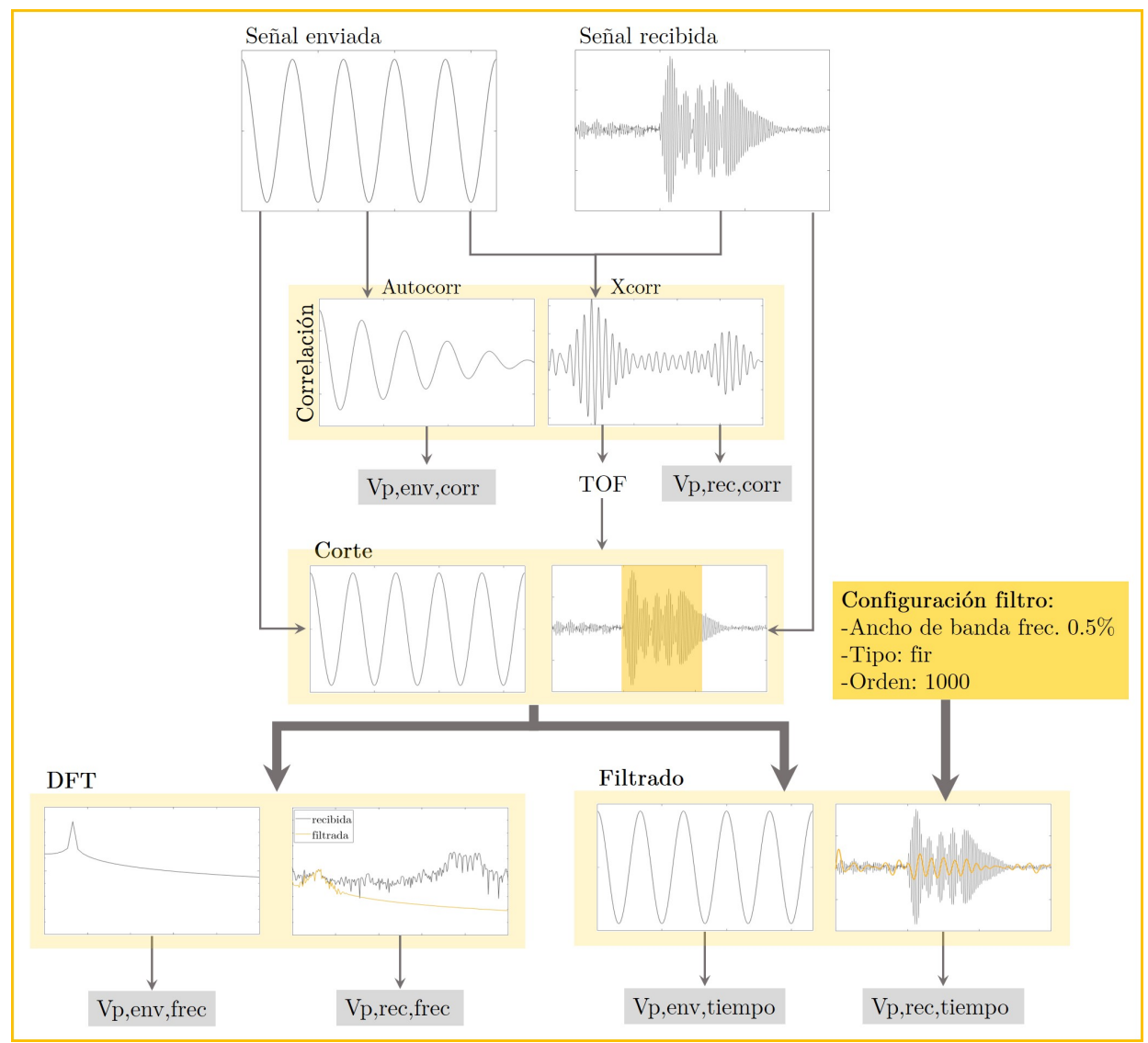

Figura 3.10: Esquema del procesado de la señal acústica no lineal 


\subsubsection{Resultados de señales paramétricas tipo seno}

Se analiza el resultado de una señal tipo seno paramétrico con frecuencia portadora en $200 \mathrm{kHz}$ y una frecuencia moduladora de $15 \mathrm{kHz}$ con una duración de $0.167 \mathrm{~ms}$. Se espera una frecuencia paramétrica de $30 \mathrm{kHz}$, que corresponde al doble de la moduladora.

\section{Análisis de señales para el haz primario}

En el siguiente análisis se estudian las señales enviadas y recibidas en el dominio de tiempo, frecuencia y correlación.

- En el dominio del tiempo, se tiene en la Figura 3.11 (izquierda), la señal enviada al sistema de grabación. Para su análisis, esta señal se cortó previamente a su duración de $0.167 \mathrm{~ms}$ con una amplitud en voltios pico de $150 \mathrm{~V}$ en las frecuencias primarias. A la derecha, se tiene la señal recibida (gris). A esta señal se le aplicó un fitro entre $195 \mathrm{kHz}$ y $205 \mathrm{kHz}$, esto es; alrededor la de frecuencia portadora. Dado que la distancia entre los transductores es de $0.35 \mathrm{~m}$, para una velocidad del sonido en el agua de $1480 \mathrm{~m}$, se espera recibir la señal directa libre de reflexiones en un tiempo de $0.23 \mathrm{~ms}$, tal como se observa en la gráfica.

- Respecto al dominio de la frecuencia, se tiene que el contenido espectral para la señal enviada corresponde a la Figura 3.11 (izquierda). A la derecha de la gráfica, está la señal recibida (gris) junto con la recibida filtrada alrededor de la frecuencia portadora (ocre).

- Respecto al domino de la correlación, la Figura 3.11 (izquierda), muestra la autocorrelación del haz primario. A la derecha, se presenta la correlación cruzada de la señal recibida filtrada alrededor de la frecuencia portadora con la señal enviada. Como es de esperar, el pico máximo de correlación se encuentra en $0.23 \mathrm{~ms}$, que corresponde al tiempo de llegada de la señal recibida.

En la Figura 3.12 se comparan las amplitudes en voltios pico de los dominios estudiados anteriormente para el haz primario de la señal seno que corresponde a una frecuencia de $200 \mathrm{kHz}$. En ella se observa que, las amplitudes en el dominio del tiempo y correlación son similares, con una menor desviación y amplitud para la de correlación respecto al dominio del tiempo. En cambio, para el dominio de la frecuencia se tiene una amplitud mucho menor; posiblemente se debe a una menor resolución espectral, sin embargo, su desviación es mínima. 

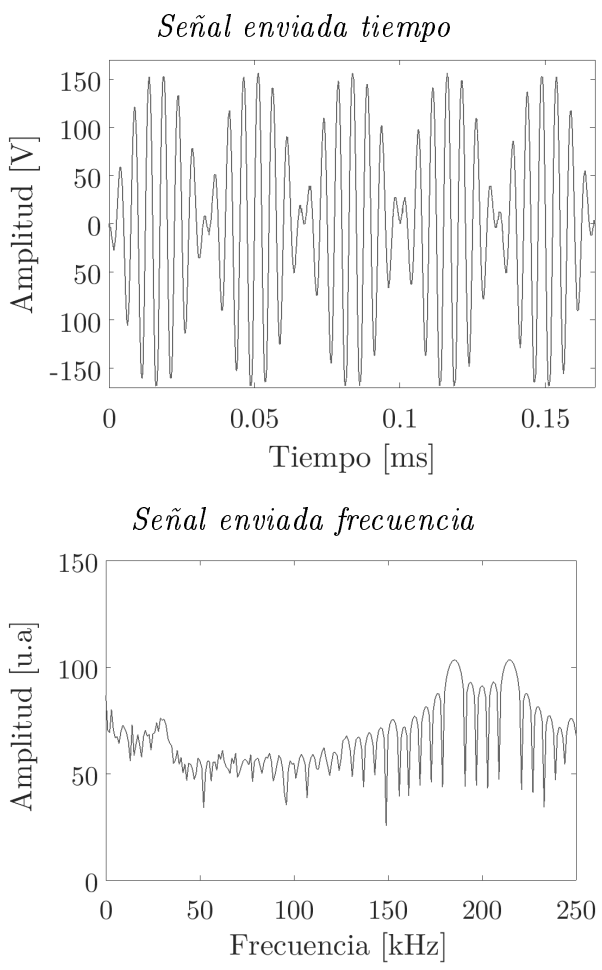

Señal enviada correlación

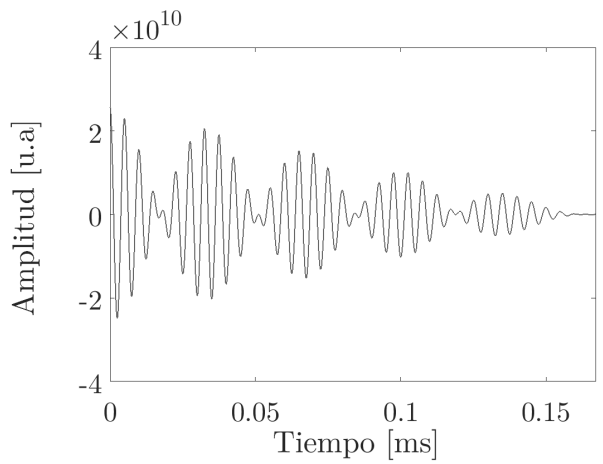

Señal recibida tiempo

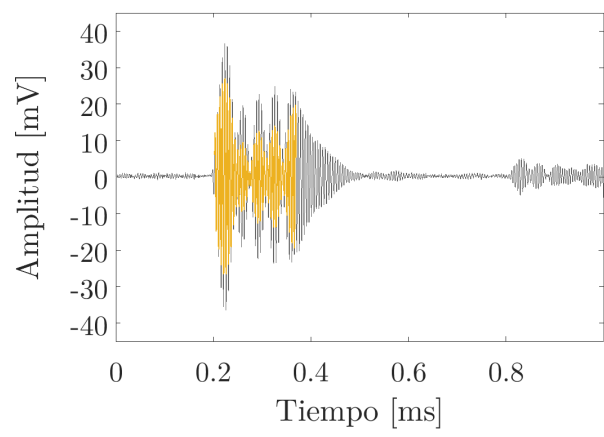

Señal recibida frecuencia

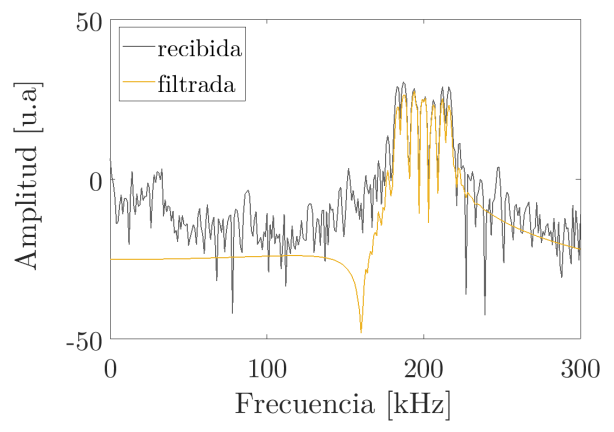

Señal recibida correlación

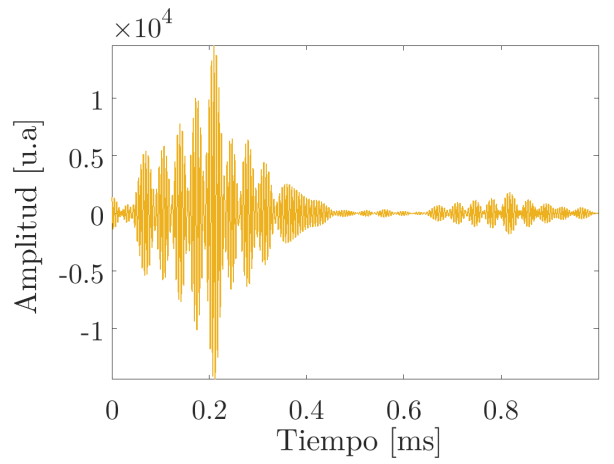

Figura 3.11: Análisis de señales tipo seno paramétrico en el dominio del tiempo, frecuencia y correlación para el haz primario 


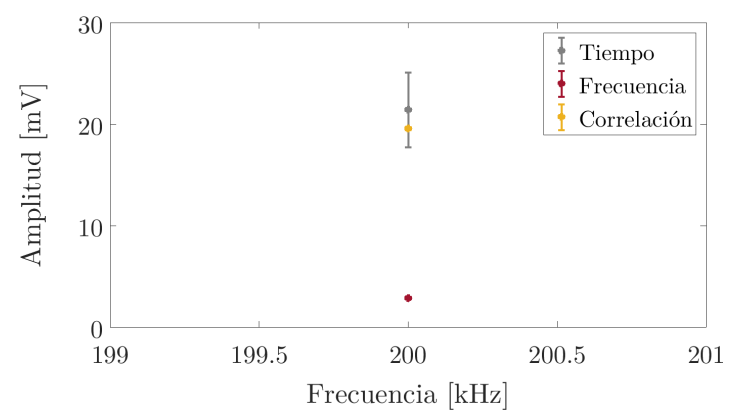

Figura 3.12: Amplitudes de los tres dominios para el haz primario del seno en $200 \mathrm{kHz} .21$ $\mathrm{mV}, 3 \mathrm{mV}$ y $20 \mathrm{mV}$ en el dominio del tiempo, frecuencia y correlación respectivamente.

\section{Análisis de señales para el haz secundario}

Se presenta un análisis similar al anterior, en lo que respecta al estudio de la señal enviada y la recibida, para el haz secundario o frecuencia paramétrica.

- En el dominio del tiempo, en la Figura 3.13 (izquierda), se tiene la segunda derivada de la envolvente al cuadrado de la señal enviada, que se utiliza para analizar la señal paramétrica. A la derecha, se tiene la señal recibida previo filtro con un orden de 20, para quitar ruido (gris), posteriormente, se filtra alrededor de la baja frecuencia (ocre) y se multiplica por un factor 15 para una mayor visualización.

- Respecto al dominio de la frecuencia, se observa en la izquierda de la Figura 3.13, un pico en la frecuencia secundaria de $30 \mathrm{kHz}$, que corresponde a la baja frecuencia paramétrica. A la derecha, el contenido espectral de la señal recibida (gris), muestra un máximo alrededor de la frecuencia portadora, y aplicando el filtro en la baja frecuencia (ocre), se obtiene el contenido espectral alrededor de los $30 \mathrm{kHz}$.

- Respecto al domino de la correlación, en la Figura 3.13 (izquierda), se presenta la autocorrelación del haz secundario. A su derecha, se presenta la correlación cruzada de la señal recibida con la segunda derivada de la envolvente al cuadrado de la señal enviada. Se obtiene un pico máximo de correlación en $0.23 \mathrm{~ms}$, tiempo que corresponde a la llegada de la señal recibida para una distancia entre transductores de $0.34 \mathrm{~m}$.

En la Figura 3.14, se comparan las amplitudes para los dominios estudiados empleando diferentes frecuencias de senos secundarios, tales como, seno de $20 \mathrm{kHz}, 30 \mathrm{kHz}$ y 40 kHz. En ella se observa que, a nivel general, el dominio del tiempo y la correlación son próximos, siendo mayor el de tiempo, excepto para la frecuencia secundaria de 40 $\mathrm{kHz}$ donde las amplitudes entre estos dos dominios son distantes. En el dominio de la 

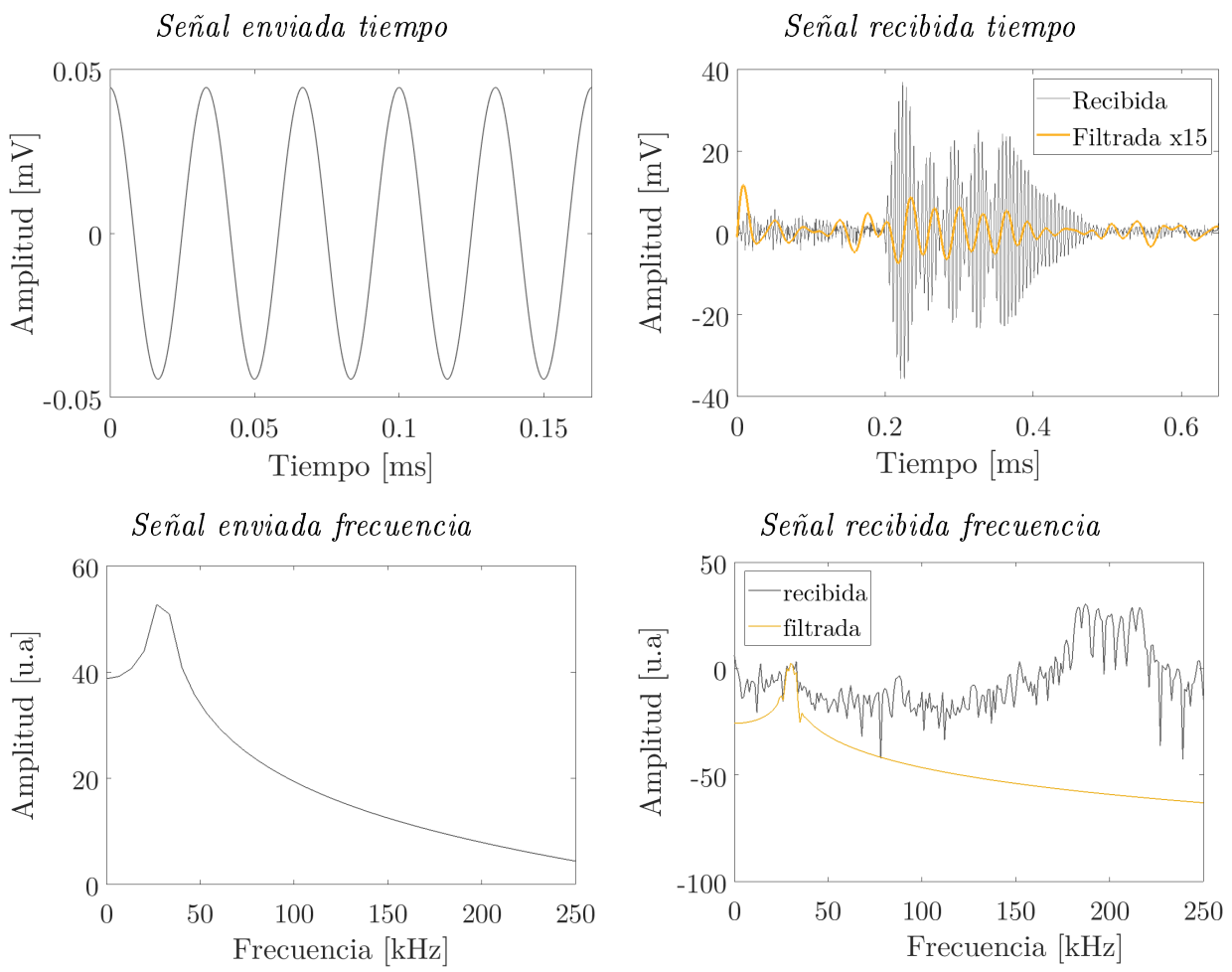

Señal enviada correlación
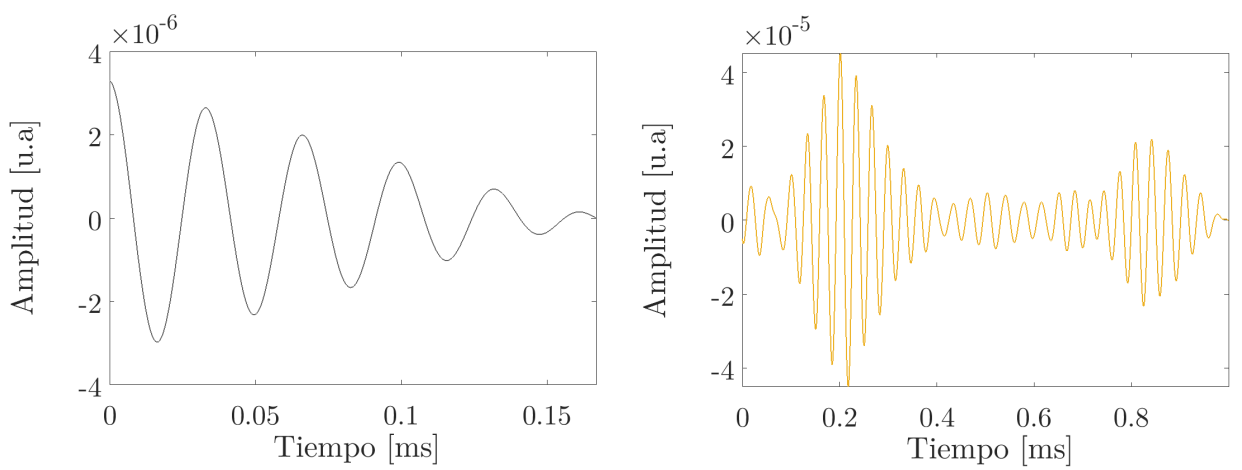

Figura 3.13: Análisis de señales tipo seno paramétrico en el dominio del tiempo, frecuencia y correlación para el haz secundario 
frecuencia en todos los casos ejemplos, se tiene una amplitud mucho menor respecto a los demás.

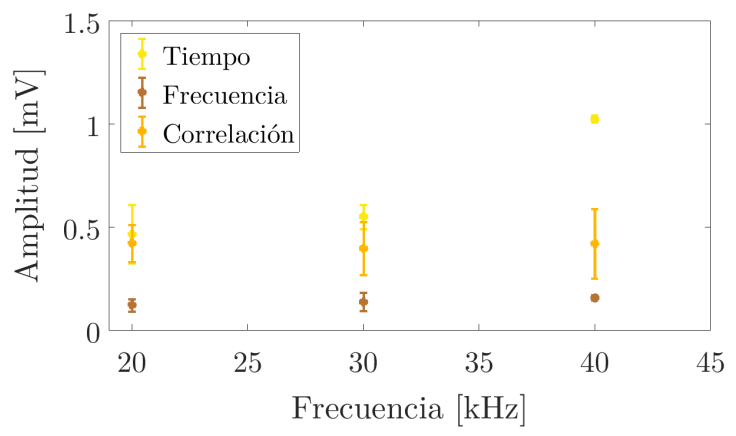

Figura 3.14: Amplitudes de los tres dominios con diferentes frecuencias de senos secundarios

\subsubsection{Resultados de señales paramétricas tipo sweep}

Se analiza un sweep paramétrico desde los $4 \mathrm{kHz}$ hasta los $40 \mathrm{kHz}$ con una frecuencia portadora en $200 \mathrm{kHz}$ y un tiempo de duración de la señal de $1 \mathrm{~ms}$.

\section{Análisis para el haz primario}

Se analiza la frecuencia portadora de $200 \mathrm{kHz}$ en el dominio del tiempo, frecuencia y correlación.

- En el dominio del tiempo, en la Figura 3.15 (izquierda), se presenta la señal enviada al sistema de grabación. Para su análisis, esta señal se cortó previamente a su duración de $1 \mathrm{~ms}$ con una amplitud en voltios pico de $41 \mathrm{~V}$ en las frecuencias primarias. A la derecha, se tiene la señal recibida (gris). A esta señal se le aplicó un filtro entre $195 \mathrm{kHz}$ y $205 \mathrm{kHz}$, alrededor la de frecuencia portadora.

- Respecto al dominio de la frecuencia, el contenido espectral para la señal enviada y recibida está a la izquierda y derecha de la Figura 3.15 respectivamente. La señal recibida está filtrada alrededor del haz primario (frecuencia portadora).

- En el domino de la correlación, la Figura 3.15 (izquierda), muestra la autocorrelación de la señal enviada, y a la derecha, la correlación cruzada de la señal recibida filtrada alrededor de la frecuencia portadora con la señal enviada. Como es de esperar, el pico máximo de correlación se encuentra en $0.21 \mathrm{~ms}$, que corresponde al tiempo de llegada de la señal recibida. 


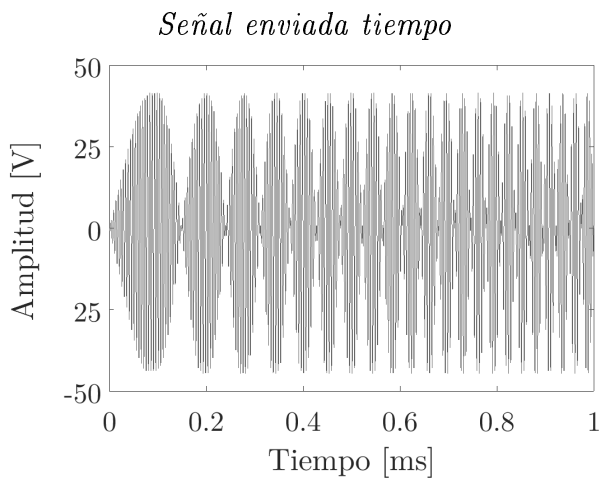

Señal enviada frecuencia

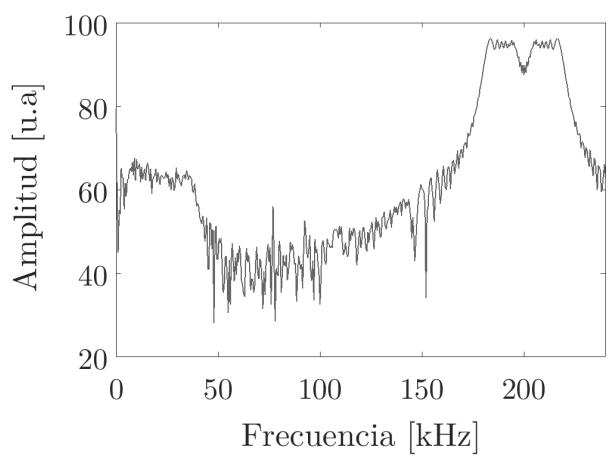

Señal enviada correlación

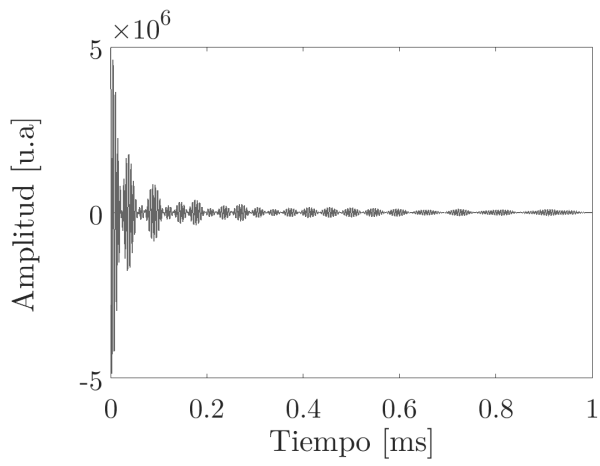

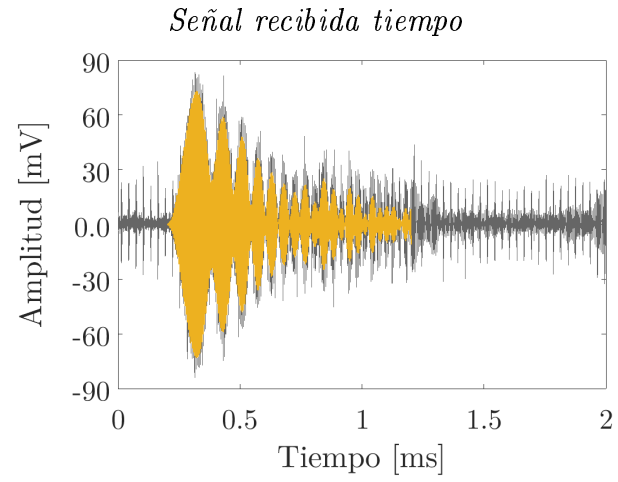

Señal recibida frecuencia

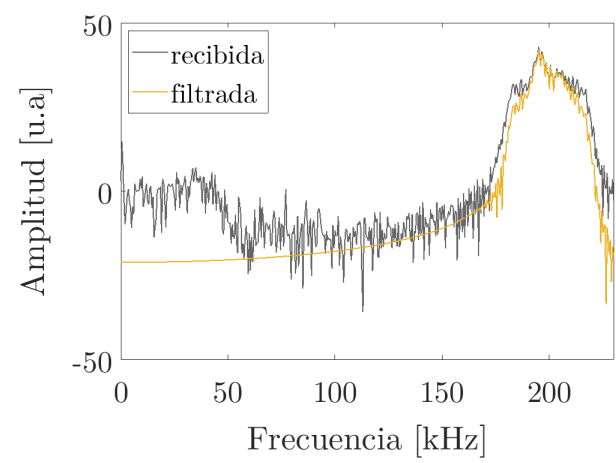

Señal recibida correlación

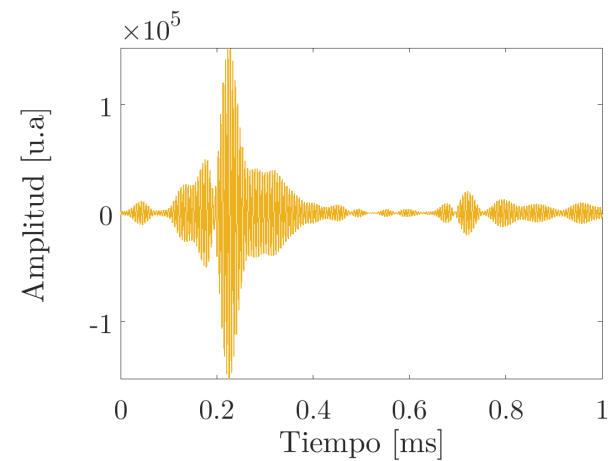

Figura 3.15: Análisis de señales tipo sweep paramétrico en el dominio del tiempo, frecuencia y correlación para el haz primario 
En la Figura 3.16, se comparan las amplitudes de los tres dominios estudiados para el haz primario que corresponde a la frecuencia portadora de $200 \mathrm{kHz}$ del sweep paramétrico. El caso es similar al estudiado para el seno del haz primario, donde las amplitudes de tiempo y correlación son cercanas entre sí y la del dominio de la frecuencia dista mucho respecto a las anteriores.

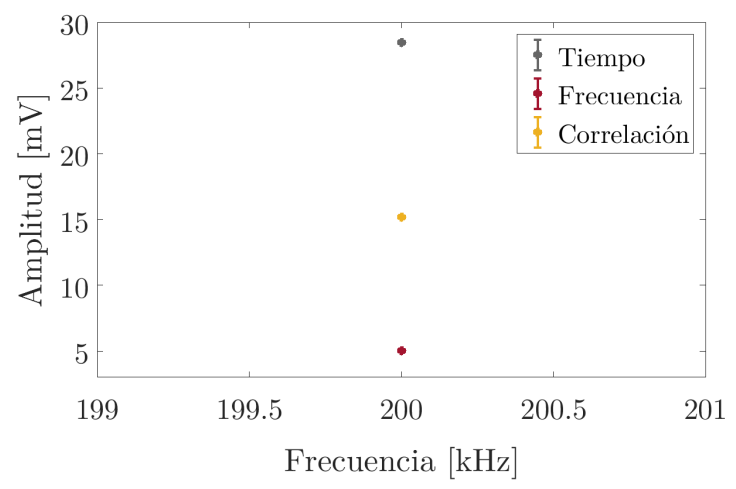

Figura 3.16: Amplitudes de los tres dominios para el haz primario del sweep en $200 \mathrm{kHz}$. $28 \mathrm{mV}$ para el dominio del tiempo, $15 \mathrm{mV}$ en correlación y $5 \mathrm{mV}$ en frecuencia

\section{Análisis para el haz secundario}

Se presenta un análisis similar al anterior, con el estudio de la señal enviada y la señal recibida, para el haz secundario.

- En el dominio del tiempo, en la Figura 3.17 (izquierda), se muestra la segunda derivada de la envolvente al cuadrado de la señal enviada, que se utiliza para analizar la señal paramétrica. A la derecha, se tiene la señal recibida previo filtro, para quitar ruido (gris). Posteriormente, se filtra alrededor de la baja frecuencia (ocre) y se multiplica por un factor 10 para una mayor visualización.

- Con el dominio de la frecuencia, se encuentra en la Figura 3.17 (izquierda), el espectro del haz secundario. A la derecha, la señal que se recibe del hidrófono (gris) para posteriormente, filtrarla alrededor de las frecuencias del sweep (ocre).

- En el domino de la correlación, en la Figura 3.17 (izquierda), se presenta la autocorrelación del haz secundario. A la derecha está, la correlación cruzada entre la señal recibida filtrada alrededor de la baja frecuencia del sweep con la segunda derivada de la envolvente al cuadrado de la señal enviada. Se tiene que el máximo de la correlación corresponde al tiempo de llegada de la señal para una distancia de $0.31 \mathrm{~m}$. 

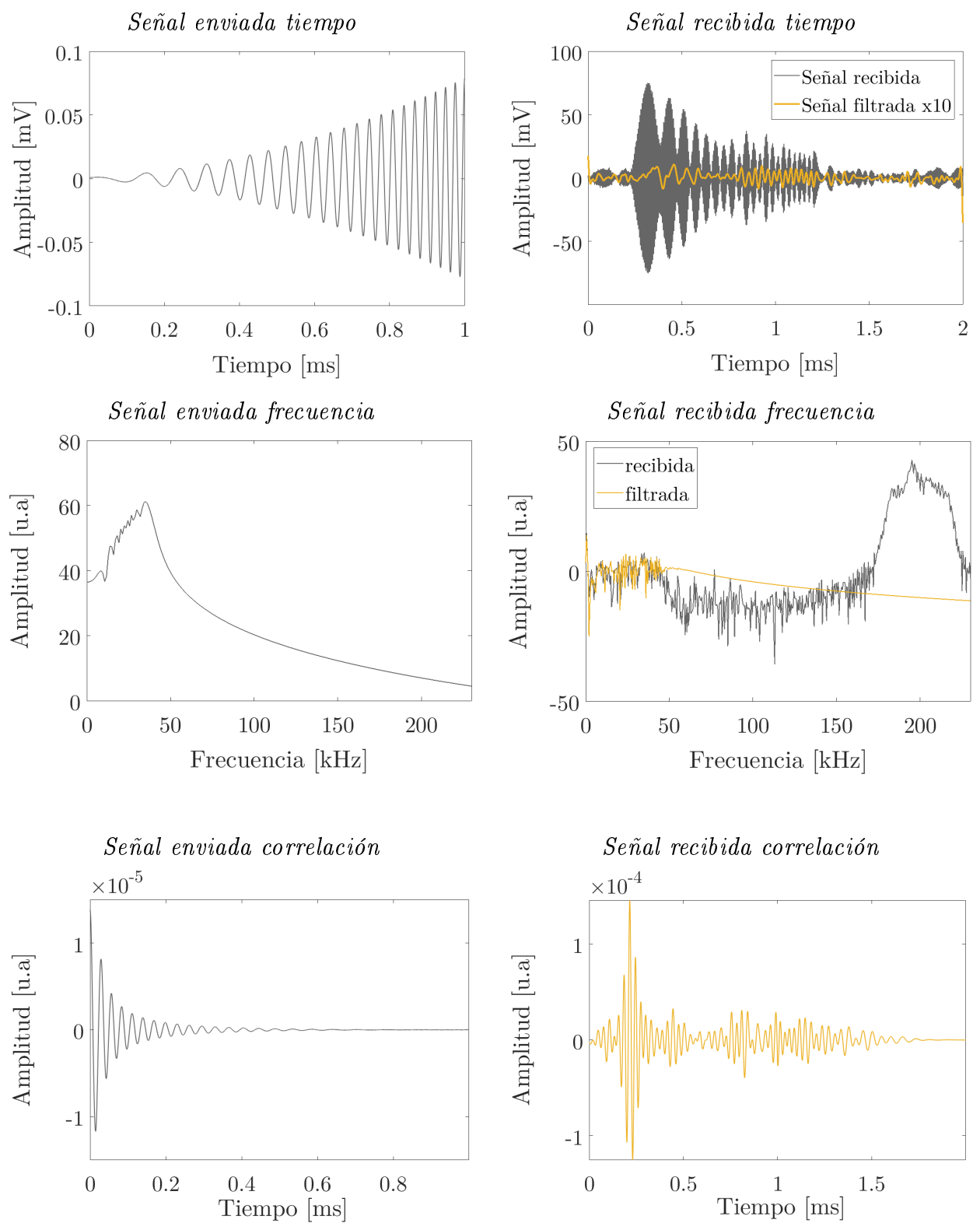

Figura 3.17: Análisis de señales tipo sweep paramétrico en el dominio del tiempo, frecuencia y correlación para el haz secundario 


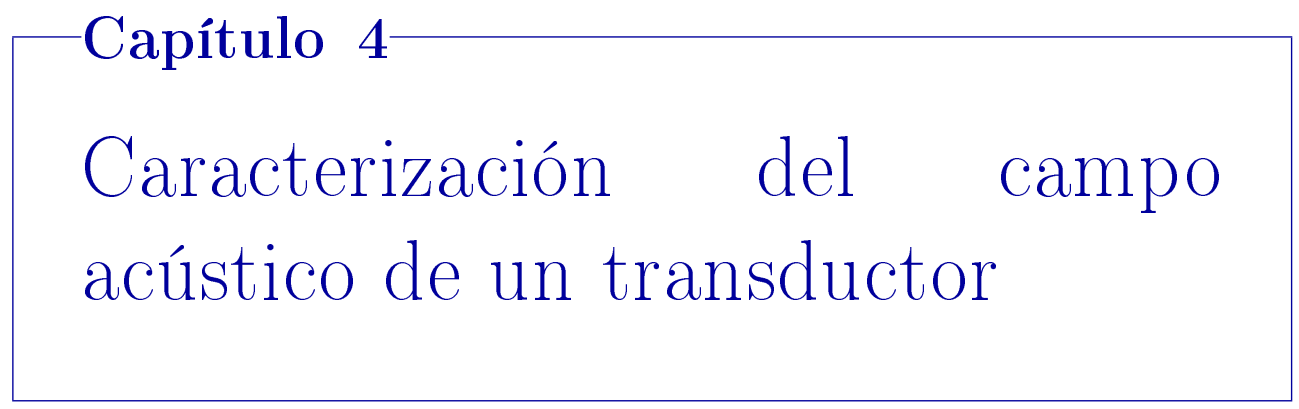

Las comunicaciones acústicas submarinas demandan el uso de transductores electroacústicos que son fundamentales para el envío y recepción de las señales acústicas. Entre ellos se tienen, los transductores proyectores (emisores) e hidrófonos (receptores). En este capítulo se presentan los experimentos hidroacústicos realizados para la calibración de los transductores empleados, y la consiguiente caracterización acústica tanto del campo lineal como no lineal (generación paramétrica), comparándolos y extrayendo las características principales de la técnica paramétrica en el uso a las comunicaciones, así como sus ventajas, empleando las técnicas de procesado vistas en el Capítulo 3.

Con lo anterior, en el apartado 4.1, se presentan los parámetros de caracterización para un transductor acústico en régimen lineal como son: la impedancia eléctrica, la sensibilidad acústica, y el patrón de directividad. Posteriormente, en el apartado 4.2, se definen los parámetros, para la caracterización en régimen no lineal que corresponde a la directividad, atenuación y variación de voltaje.

En el apartado 4.3, se muestra el montaje utilizado para las medidas experimentales.

Por último, en el apartado 4.4, y apartado 4.5, se presentan los resultados experimentales de caracterizar el transductor en régimen lineal y no lineal.

Así pues, en este capítulo se presentan los resultados de las características de la generación paramétrica al utilizar las diferentes modulaciones estudiadas en el capítulo anterior basadas en la generación de senos (tonos) y barridos frecuenciales (sweeps). 


\subsection{Parámetros de caracterización en régimen lineal}

\section{Impedancia eléctrica}

El comportamiento electromecánico de un transductor se puede entender recurriendo a diversos modelos de circuitos equivalentes, en particular, el de la impedancia eléctrica, factor determinante para el diseño de los transductores, que depende del material de la cerámica piezoeléctrica y sus capas de adaptación delanteras (machine layer) y traseras (backing). La impedancia eléctrica está dada por $(Z=V) / I$ donde el parámetro $V$, es el voltaje a través de las entradas eléctricas del transductor e $I$, es la intensidad de corriente a través de los electrodos. Dado que puede existir un desfase entre ambas magnitudes, la impedancia eléctrica es, en general, una magnitud compleja.

Como la impedancia es la oposición de los circuitos (el transductor) al paso de la corriente alterna, en el caso de proyectores, resulta interesante trabajar con la admitancia eléctrica, que es la facilidad que ofrece un circuito al paso de la corriente, ya que, desde un punto de vista práctico, la información acerca del comportamiento de la frecuencia de resonancia $f_{r}$ de la cerámica, en relación a su sensibilidad acústica, está relacionada en este valor.

Un comportamiento del valor absoluto de la admitancia se observa en la Figura 4.1.

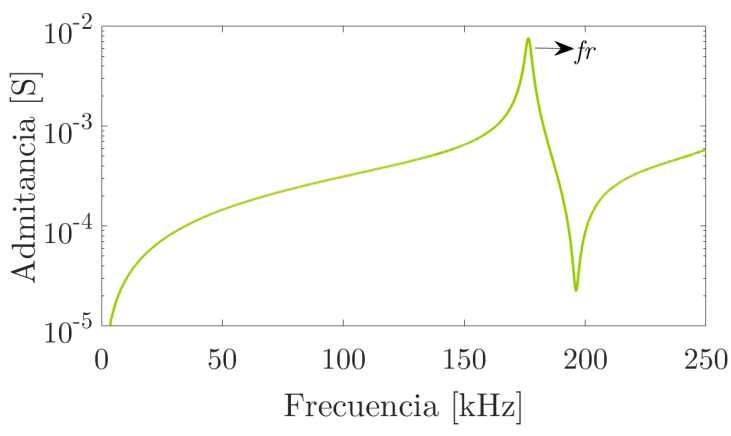

Figura 4.1: Ejemplo de admitancia eléctrica 


\section{Sensibilidad acústica}

La sensibilidad expresa la relación entre los valores de entrada y salida de un transductor; es decir, la presión acústica y el voltaje eléctrico o viceversa, en función de si se trata de un transductor o de un hidrófono.

La sensibilidad en emisión o $\mathrm{TVR}^{1}$. Expresa la relación entre la presión $P$ generada y medida a 1 metro de distancia de la fuente cuando se aplica una tensión en voltios $V$ en bornes del transductor. Estos valores son positivos y cuanto mayores son, más sensible será el transductor. El TVR caracteriza a los transductores que se emplean para emitir señales acústicas y se calcula con (4.1). En la Figura 4.14, se muestra la medida de sensibilidad para el transductor Airmar P19 que se emplea en las medidas de calibración.

$$
T V R=20 \log \left(P_{1 m} / V_{i n}\right) \quad[d B \text { re } \mu P a / V]
$$

La sensibilidad en recepción o $\mathrm{RVR}^{2}$. Es la relación entre la tensión de salida que genera el hidrófono, $V_{\text {out }}$, si sobre él incide una determinada presión $P$, cuando está en circuito abierto. Estos valores son negativos, y cuanto menos, más sensibles será el hidrófono. Se calcula mediante (4.2):

$$
R V R=20 \log \left(V_{\text {out }} / P\right) \quad[d B \text { re } V / \mu P a]
$$

En la Figura 4.2, se tiene el RVR del hidrófono Reson TC4034 con un valor de $-218 d B$ re $V / \mu P a$.

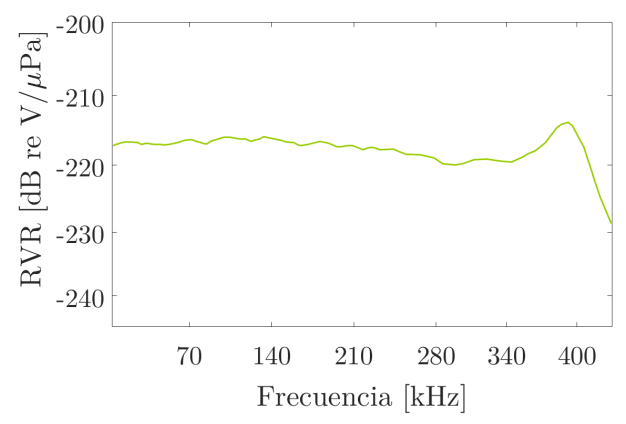

Figura 4.2: RVR del hidrófono Reson TC4034

\footnotetext{
${ }^{1}$ Transmitting Voltage Response

${ }^{2}$ Receiving Voltage Response
} 
Al igual que la admitancia, ambos parámetros TVR y RVR, son magnitudes dependientes de la frecuencia y en algunos casos, interesará que dicha respuesta sea plana o presente una mayor sensibilidad en un rango frecuencial a costa de perderla en otros.

\section{Directividad}

Cuantifica la relación entre la amplitud de la presión acústica que se genera en una dirección de interés respecto a la presión acústica sobre una dirección de referencia (comúnmente utilizando eje acústico, o dirección máxima de radiación) [90]. Para un tamaño de transductor dado, esta depende de la frecuencia, la distancia y del ángulo respecto del eje de radiación:

- Respecto a la frecuencia: en bajas frecuencias el transductor es omnidireccional (independiente del ángulo) ya que la longitud de onda es grande en comparación con el transductor; en cambio, en altas frecuencias su comportamiento es directivo, habrá presencia tanto del lóbulo principal como de los laterales.

- Respecto a la distancia: la directividad del transductor es independiente después de alcanzar la distancia del campo lejano donde comienza la divergencia esférica de la presión acústica radiada. Por el contrario, en campo cercano, las interferencias debidas a la proximidad de la fuente hacen depender la directividad de la posición en cuestión.

- Respecto al ángulo: este depende de la longitud de la onda acústica y del tamaño del transductor. Para determinar el ángulo de cobertura de un transductor, se debe hablar del ancho del haz, que es aquel ángulo en que el nivel de presión cae $3 \mathrm{~dB}$ respecto al nivel en el eje y el centro de la fuente [91,92]. Para haces del mismo ancho las bajas frecuencias necesitarán de un transductor más grande.

Para un transductor plano circular de radio $r$, como el que se utiliza en esta tesis, la función de directividad en campo lejano $(r \gg \lambda)$, se calcula a partir de la siguiente expresión:

$$
D(\theta, \varphi)=\left(\frac{2 \cdot J_{1}(k \cdot r \cdot \sin \theta)}{k \cdot r \cdot \sin \theta}\right)^{2}
$$

Donde $J_{1}$ es la función de Bessel de primer orden, $k$, es el número de onda, y $r$ es el radio del pistón. En ella, se puede ver que la directividad de una fuente está relacionada con la frecuencia de trabajo del transductor así como con el tamaño del mismo. A medida que aumenta el parámetro $k a$, mayor es la directividad del transductor. A 
partir de la expresión (4.3), se tiene el ancho del haz. En la Figura 4.3 se muestra un ejemplo del patrón de directividad de un pistón plano. En $200 \mathrm{kHz}$ se tiene un ancho de haz de $\pm 8^{\circ}$ y en las frecuencias de 10,15 y $30 \mathrm{kHz}$ las directividades presentan un ancho de haz mayor a $\pm 60^{\circ}$.

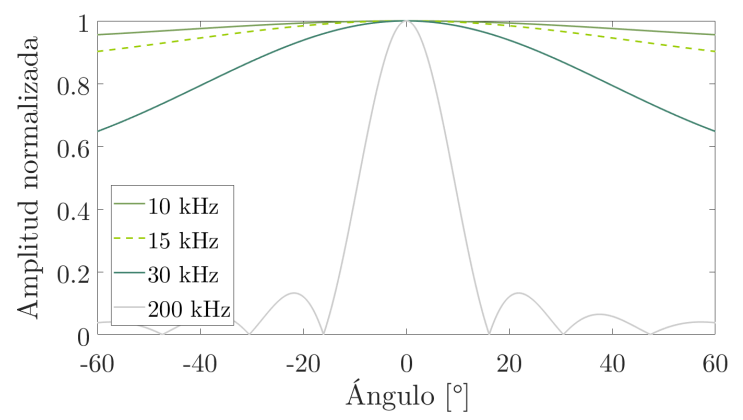

Figura 4.3: Patrón de directividad de un pistón plano con $r \quad 33 \mathrm{~mm}$ para 10, 15, 30 y $200 \mathrm{kHz}$

\subsection{Parámetros de caracterización en régimen no lineal}

\section{Directividad}

En el apartado anterior se describió la directividad, en esta sección cabe únicamente señalar que la directividad del paramétrico (haz secundario) presentará anchos de haces más estrechos respectos de los que cabría esperar para dichas frecuencias secundarias si se emitieran directamente desde el transductor. De hecho, como se verá, la directividad del haz de baja frecuencia secundario será más similar a la del haz de alta frecuencia primaria.

Un ejemplo de directividad de un haz paramétrico se muestra en la Figura 4.4. Como se observa, estas frecuencias relativamente bajas presentan anchos de la directividad cercanos $\mathrm{a} \pm 1^{\circ}$ contrastados con los anchos mayores a $\pm 60^{\circ}$ que se obtenían en las directividades lineales para estas mismas frecuencias. 


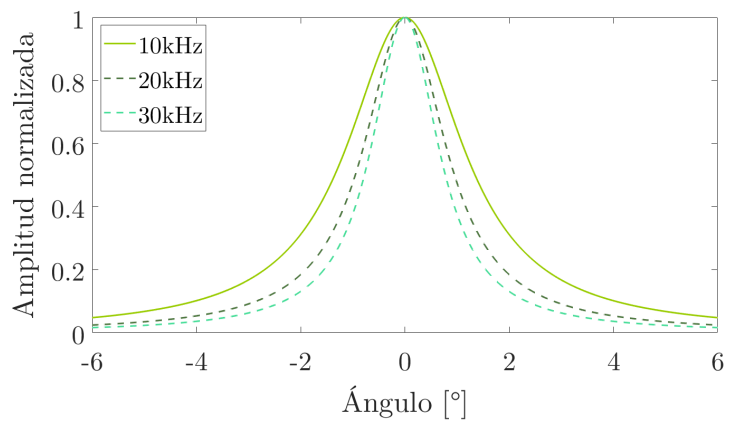

Figura 4.4: Directividad del paramétrico

\section{Atenuación}

La atenuación cuantifica la amplitud de la presión en función de la distancia. Para estudiarla, se parte de una distancia inicial entre transductores al comienzo del campo lejano y posteriormente se van alejando con distancias equidistantes entre sí. En cada una de las distancias, se emite la señal a través del transductor proyector y se recibe por el hidrófono. Con esto, se analiza la generación del haz primario y el haz secundario. La atenuación se produce por diferentes factores, desde la propia divergencia y absorción de las señales en el medio, hasta las diversas reflexiones que puedan producirse por una inhomogeneidad del medio. La atenuación es directamente proporcional a la frecuencia empleada, por lo que se debe esperar una mayor pérdida de la intensidad con ondas de mayor frecuencia. Esto se puede evidenciar en la Figura 4.5, donde se presenta un ejemplo tanto para el haz primario y secundario.

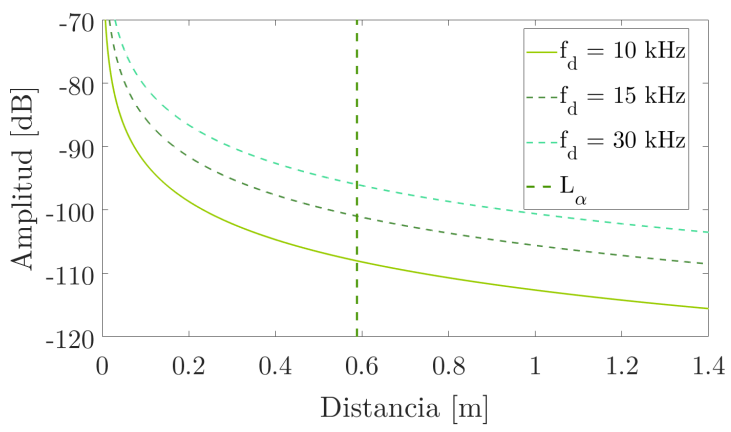

Figura 4.5: Atenuación del paramétrico. En 0.60 m empieza el campo lejano

De la atenuación se tiene que, la amplitud de la presión disminuye $6 \mathrm{~dB}$ de forma inversa al duplicar la distancia, y la amplitud de la frecuencia paramétrica depende 
cuadráticamente con dicha frecuencia, de modo que, el nivel de presión es mayor para frecuencias elevadas del paramétrico, aunque estos valores dependen de la distancia a la que se forma el campo lejano.

\section{Variación de voltaje}

Una característica del efecto paramétrico es que la amplitud del haz secundario no aumenta proporcionalmente con la del haz primario, en contraste con la linealidad que sí existe en un campo acústico lineal. La variación de voltaje estudia la amplitud en función de la tensión aplicada a la señal que se emite, con lo cual también se podrá distinguir un valor en tensión aproximado donde se crea dicho efecto. Se comparan las amplitudes del haz primario (que crece de forma lineal) y el secundario (crecimiento parabólico) emitiendo la señal en diferentes valores de voltaje.

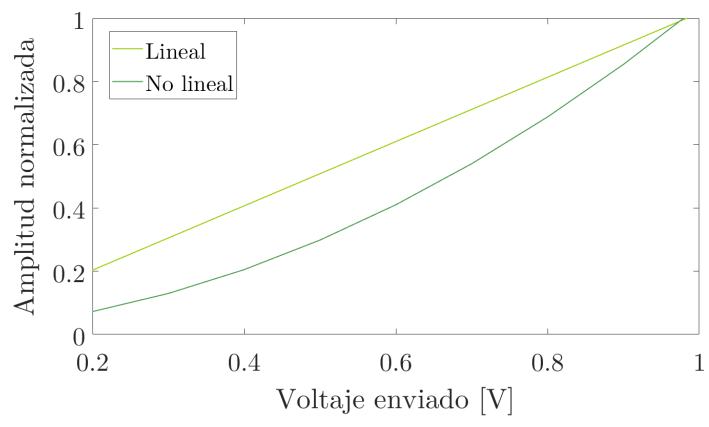

Figura 4.6: Ejemplo de variación de voltaje

\subsection{Montaje experimental}

En este apartado, se describen los diferentes montajes realizados en piscina y balsa para todos los ensayos estudiados en cuestión, tanto en régimen lineal como en no lineal. En régimen lineal, se realiza el montaje de impedancia eléctrica y sensibilidad en emisión TVR. En régimen no lineal, se tiene un montaje para las medidas de directividad, atenuación y variación de voltaje. Estas medidas, se realizan empleando las instalaciones (laboratorio de hidroacústica, balsa y sistema de posicionamiento) del Centro Tecnológico Naval y del Mar (CTN).

En esta primera etapa, se emplea una configuración que utiliza un transductor plano Airmar P19 como emisor, en un entorno de laboratorio controlado. El objetivo de este experimento es, calibrar el sistema para garantizar una medición de calidad. 


\subsubsection{Régimen lineal}

\section{Montaje de impedancia eléctrica}

La Figura 4.7, presenta el montaje donde se emplea el medidor de impedancias de alta frecuencia Keysight E4980AL, se envía un barrido frecuencial desde los $10 \mathrm{kHz}$ hasta los $250 \mathrm{kHz}$ en pasos de $0.1 \mathrm{kHz}$ y una tensión de amplitud de $1 \mathrm{~V}$. A través de la diferencia de potenciales, se mide la respuesta que devuelve el transductor. Por último, se guardan los datos registrados en un archivo tipo texto (.txt) para su posterior procesado.
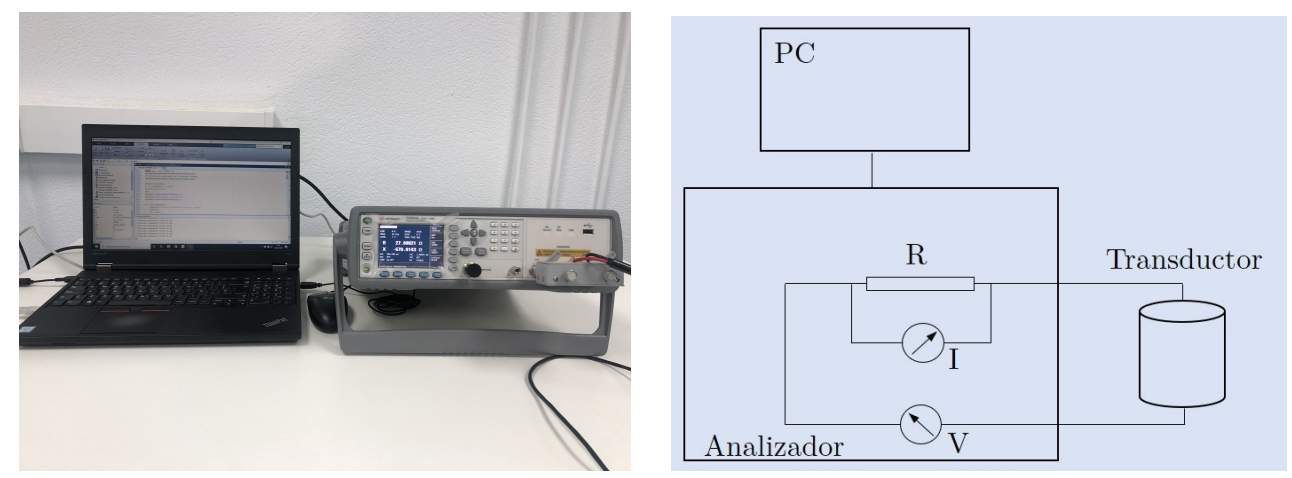

Figura 4.7: Imagen del montaje experimental del medidor de impedancia (izquierda) y diagrama de bloques con funcionamiento interno del medidor (derecha)

\section{Montaje de TVR en piscina}

Con estos equipos, se realizaron los ensayos correspondientes a la caracterización de sensibilidad TVR y los primeros experimentos para la caracterización en régimen no lineal tales como, directividad, atenuación y variación de voltaje.

La Figura 4.8, muestra el montaje y conexionado para la calibración. Se utiliza el amplificador 1040L de E\&I, el sistema de generación y adquisición de datos con chasis PXI-1073 de National Instruments, y tarjeta generadora PXIe-5433 y osciloscopio PXIe-5122, la tarjeta NI PXI ExpressCard-8360 que se conecta a un computador portátil y una sonda atenuadora x100 de TES TECT serie HV, cuyo fin es atenuar la señal a la salida del amplificador para que se pueda registrar en el PXI. Las medidas fueron desarrolladas en una piscina cilíndrica de dimensiones de $4 \mathrm{~m}$ de diámetro por 1.50 metros de alto y a una distancia entre transductores de $0.44 \mathrm{~m}$. El hidrófono Reson TC4034 ubicado en la izquierda de la piscina tiene una sensibilidad en recepción 

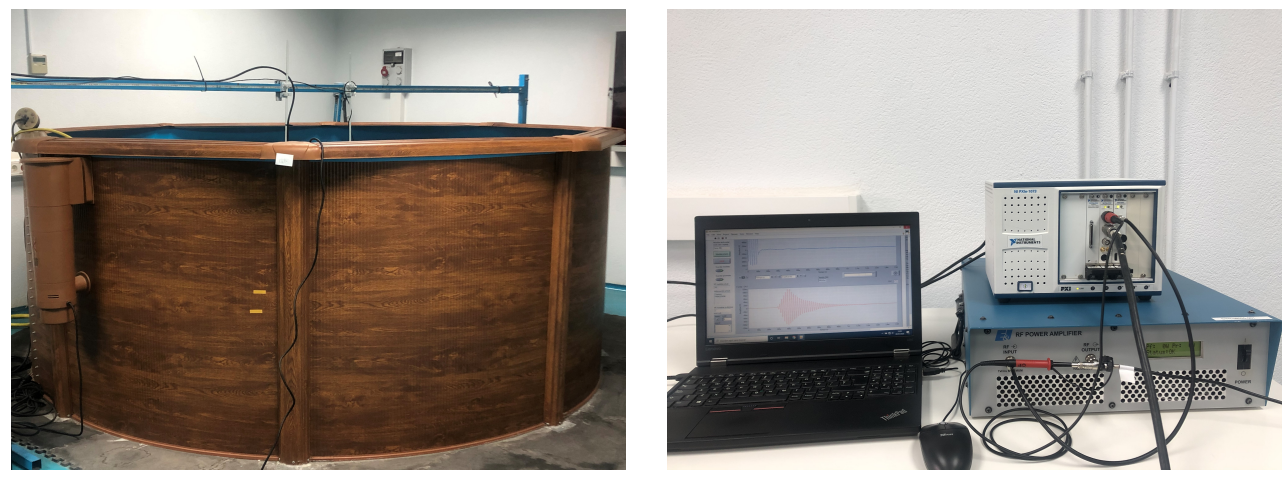

Figura 4.8: Montaje para la medida de TVR con una distancia entre transductores de 0.44 $\mathrm{m}$ en la piscina (izquierda). Equipos y conexionado empleados (derecha)

de $-218 d B$ ref $1 V / \mu P a @ 1 m$ en su ancho de banda frecuencial. A la derecha de la piscina se ubica el emisor Airmar P19. En la Figura 4.9, se muestra el esquema del montaje de calibración del TVR.

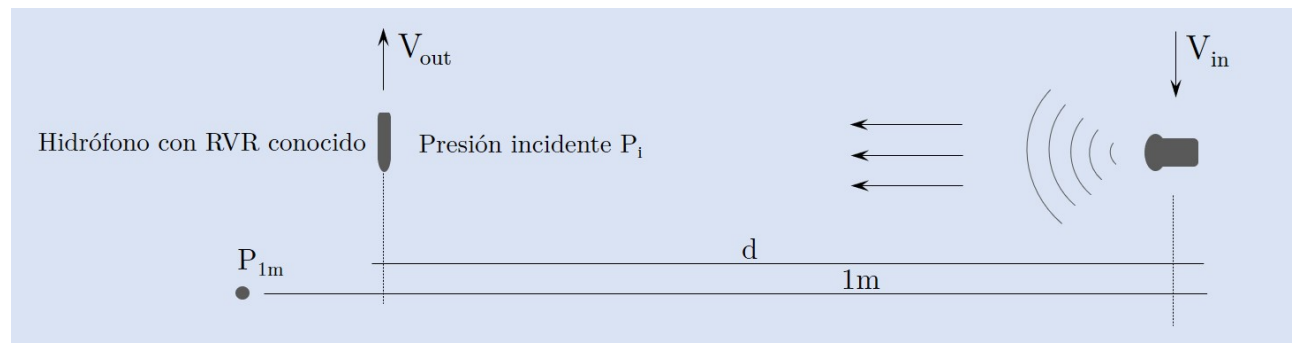

Figura 4.9: Esquema del montaje de calibración del TVR

Para la obtención del TVR, se emite una señal senoidal de 5 ciclos de duración con una frecuencia inicial de $10 \mathrm{kHz}$ hasta $250 \mathrm{kHz}$ en pasos de $1 \mathrm{kHz}$, realizando 10 muestras para cada frecuencia. Se aplica una amplitud en voltios pico a pico de 600 $m V_{p p}$ con una frecuencia de muestreo de $2 \mathrm{MS} / \mathrm{s}$. Las tensiones de la señal enviada $V_{i n}$, son registradas por el emisor (transductor a caracterizar) y las recibidas $V_{\text {out }}$, por el hidrófono (transductor de referencia). Conociendo el RVR del hidrófono de referencia, se calcula la presión $P_{i}$ que incide en él, y se corrige por propagación esférica a la distancia de referencia de $1 \mathrm{~m}, P_{1 m}$. 


\subsubsection{Régimen no lineal}

\section{Montaje en piscina}

Se utiliza el transductor Airmar P19 y el hidrófono Reson TC4040, que posee una sensibilidad en recepción RVR de $-206 \mathrm{~dB}$ re $\mathrm{V} / \mu \mathrm{Pa}$, la distancia entre transductores es de $0.35 \mathrm{~m}$. En la Figura 4.10, se presenta dicho montaje.
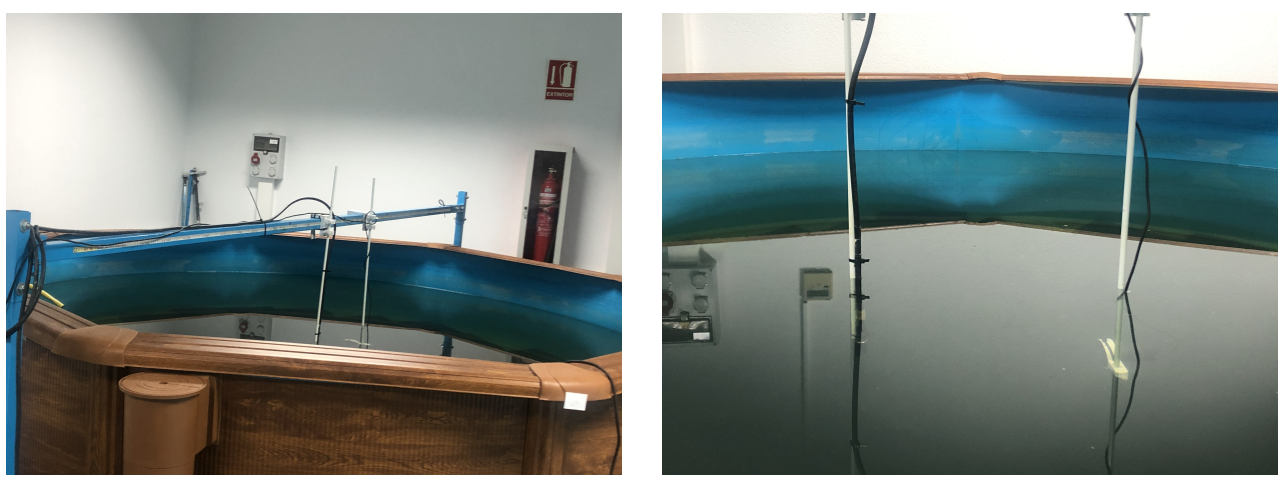

Figura 4.10: Montaje experimental para medidas en régimen no lineal. A la izquierda de la piscina, el hidrófono Reson TC4040 y a la derecha el transductor Airmar P19

\section{Montaje en balsa de calibración}

En la Figura 4.11, se presenta el montaje donde se desarrollaron las medidas en emisión de las señales a mayores distancias, utilizando la balsa con geometría troncocónica de 10 metros de profundidad, y 20 metros de diámetro junto con el sistema de posicionamiento SMARLOGY, eje X servo motor BMH1003P32F2A, eje Y servo motor BMH1003P32F2A.

\subsubsection{Configuración de las medidas}

La Figura 4.12, es un diagrama de bloques del montaje experimental desarrollado en todas las caracterizaciones; es decir, en régimen lineal: sensibilidad y directividad y en régimen no lineal (paramétrico): directividad, atenuación y variación de voltaje.

Las medidas se realizaron con el generador de funciones PXIe 5433 de NI, que conectado al PC y a través de LabVIEW, envía la señal seleccionada por el usuario hacia la entrada del amplificador 1040L RF de E\&I, con el fin de aumentar la amplitud de la señal, y por medio de su salida, enviarla a un transductor piezoeléctrico. A su 

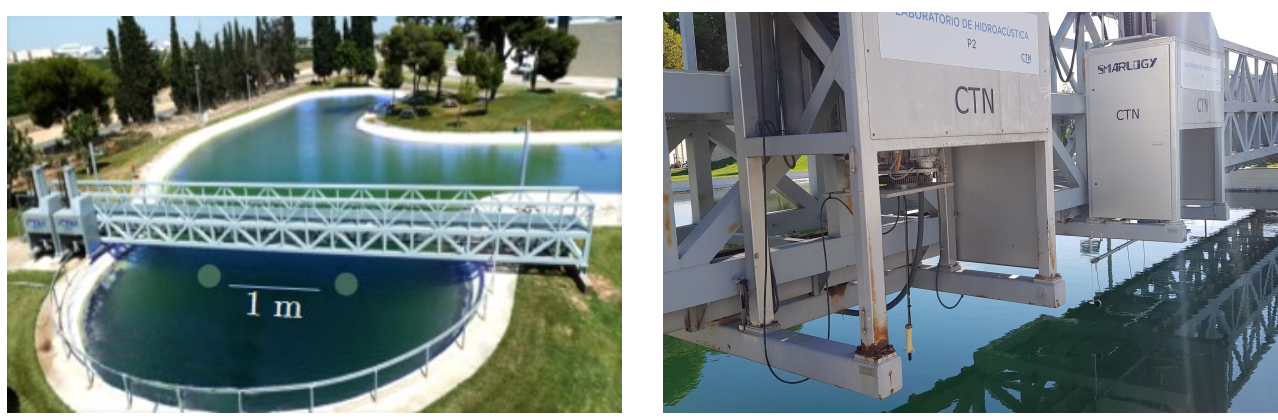

Figura 4.11: Montaje experimental en el Centro Tecnológico Naval y del Mar CTN. Balsa y ubicación de los transductores (izquierda) y sistema de posicionamiento (derecha)

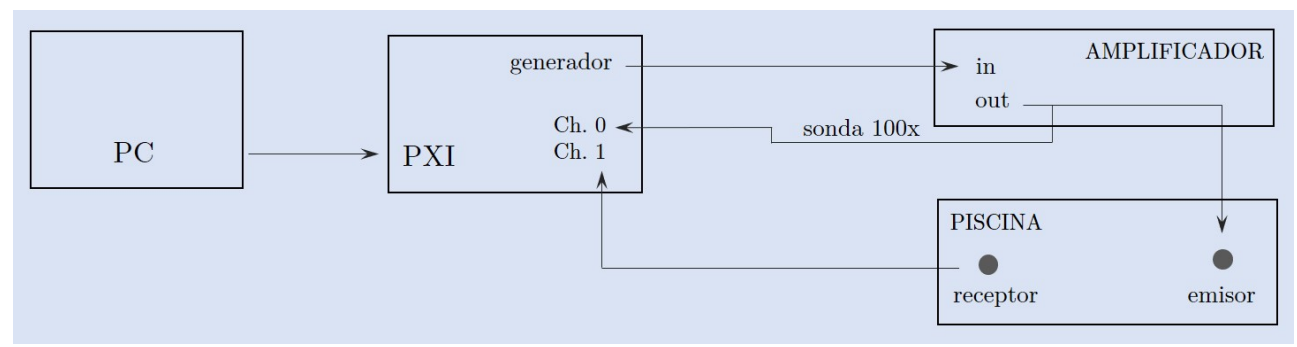

Figura 4.12: Diagrama de bloques del montaje y conexionado para la caracterización en régimen lineal (TVR) y no lineal (directividad, atenuación y variación de voltaje)

vez, en la misma salida del amplificador, se conecta una sonda que atenúa la señal 100 veces para que se pueda registrar en la entrada del PXI a través del canal 0 (ch. 0). Las ondas de presión propagadas en el agua se reciben por el hidrófono que se convierten posteriormente en tensión eléctrica, y se registran a la entrada del canal 1 (ch. 1) del PXI. El programa de LabVIEW genera un conjunto de ficheros de las señales enviadas y recibidas que se analizan con el software MATLAB. Para los ensayos de directividad, atenuación y voltaje en la balsa del CTN se emplean los posicionadores SMARLOGY. 


\subsection{Resultados de caracterizar un transductor en régimen lineal}

\subsubsection{Admitancia}

El transductor emisor empleado para emitir las señales acústicas tanto en el régimen lineal como en régimen no lineal (paramétrico) es el Airmar P19. En la Figura 4.13, se muestra la gráfica de la admitancia.
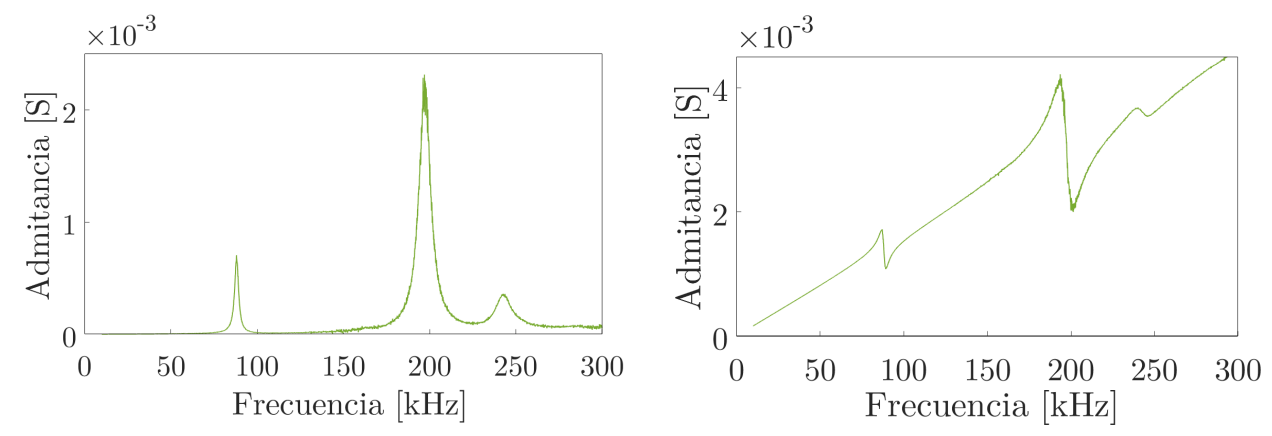

Figura 4.13: Gráfica de la admitancia eléctrica para el transductor Aimar P19. Magnitud (izquierda) y fase (derecha)

Respecto a la gráfica de la magnitud, se obtiene para el modo en espesor (alta frecuencia) un máximo de $200 \mathrm{kHz}$ en la frecuencia de resonancia. Además, se tiene la presencia del modo radial (baja frecuencia), con una frecuencia de resonancia de $80 \mathrm{kHz}$, pero con una menor amplitud. Esto se puede corroborar, con la gráfica de la fase. Además, presenta otra frecuencia de resonancia en $244 \mathrm{kHz}$ en el modo espesor, pero con menor influencia en la amplitud.

En el siguiente apartado, se contrasta el valor de la admitancia para la frecuencia de resonancia de $200 \mathrm{kHz}$ con las medidas de TVR, donde se tiene que justo para esta frecuencia existe una máxima sensibilidad.

\subsubsection{TVR}

En esta tesis, se utiliza el método por comparación directa [17], que consiste en comparar la salida de un transductor desconocido con la de un transductor de referencia calibrado previamente.

Para las medidas de sensibilidad en emisión TVR se emplea como transductor de referencia, el hidrófono Reson TC4034 por poseer una respuesta en frecuencia plana en 
todo su rango frecuencial, presentado en la Figura 4.2. Una vez realizado los ensayos, se obtiene la sensibilidad en emisión en la Figura 4.14.

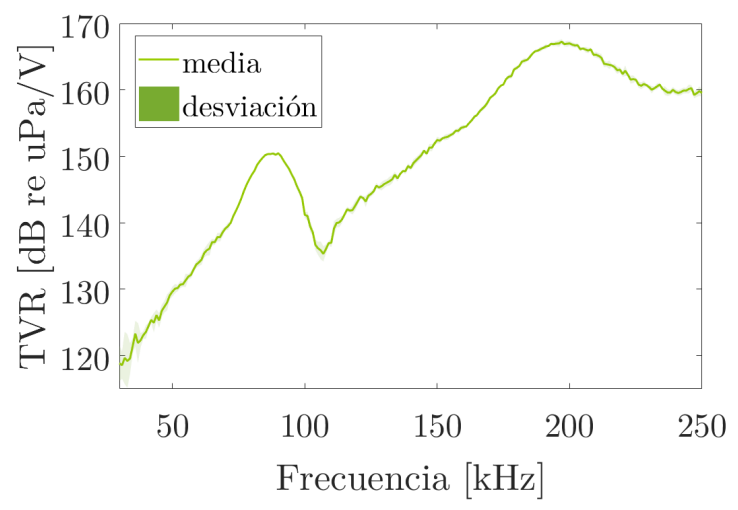

Figura 4.14: Gráfica de TVR para el transductor plano Airmar P19

En la Figura 4.14 se tiene que, para la alta frecuencia; modo de vibración en espesor de $200 \mathrm{kHz}$, el transductor Airmar P19 presenta una sensibilidad en emisión de $167 d B$ re $V / \mu P a @ 1 m$. Como se observa, la frecuencia de resonancia obtenida a través de la admitancia eléctrica en $200 \mathrm{kHz}$ se obtiene una máxima sensibilidad en emisión. Con esta frecuencia se modulan las señales paramétricas y así emplear el transductor en una emisión óptima.

\subsection{Resultados de caracterizar un transductor en régimen no lineal (paramétrico)}

El desarrollo acústico paramétrico, se evaluó mediante la generación paramétrica de una señal tipo seno y otra de tipo sweep (barrido senoidal) utilizando la técnica de la correlación cruzada, explicada en el Capítulo 3.

En todos los experimentos descritos en este apartado, la frecuencia portadora $f_{p}$, (alta frecuencia) corresponde a la frecuencia de resonancia de $200 \mathrm{kHz}$ del transductor Airmar P19.

En los siguientes apartados, se detallan los resultados de los estudios que confirman la generación del efecto paramétrico para las señales estudiadas en cuestión explicadas en el apartado 4.2 como son: directividad, atenuación y variación de voltaje. 


\subsubsection{Medidas con senos paramétricos}

Se estudia la generación paramétrica de una señal senoidal en el tiempo, explicada en el Capítulo 3 para un seno modulado en $15 \mathrm{kHz}$ con una duración de $1 \mathrm{~ms}$, y otro en $20 \mathrm{kHz}$ a $500 \mu \mathrm{s}$. Con ello, se obtienen las frecuencias diferencias de 30 y $40 \mathrm{kHz}$. Los análisis de los diferentes estudios paramétricos con estas señales se obtuvieron tomando el valor de amplitud de correlación de la señal recibida con la señal del haz primario (alta frecuencia) y con la señal del haz secundario esperado (baja frecuencia).

\section{Directividad}

Se analiza el haz primario y el secundario experimentalmente. Luego, utilizando la minimización de la desviación cuadrática media de los datos experimentales, se ajustan al modelo de la expresión (4.3) del pistón plano, ya que para el haz primario (lineal), la directividad del lóbulo principal viene dada a través de esa expresión y como la directividad del haz secundario tiende a ser parecida a la del primario, entonces es adecuado utilizar esta expresión como modelo para ambos haces. Partiendo de (4.3) se realiza el ajuste tomando un radio acústico teórico del transductor de $1.44 \mathrm{~cm}$ (valor muy parecido al radio real de la cerámica con $1.65 \mathrm{~cm}$ ) y variando la frecuencia, se obtiene el ajuste con una frecuencia equivalente para cada haz.
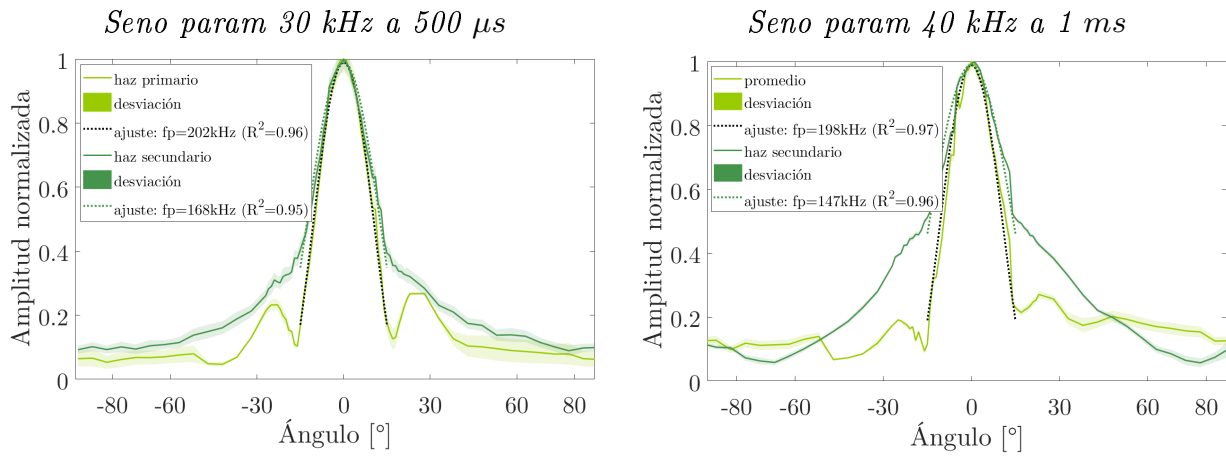

Figura 4.15: Directividad para senos paramétricos. Valores de normalización para el haz primario de $13 m V_{\text {pico }}$ y para el secundario de $0.55 m V_{\text {pico }}$

En la Figura 4.15 se observa, que las directividades son similares a pesar de las diferencias espectrales para el haz primario (alta frecuencia) y el haz secundario (bajas frecuencias). En ambos senos, se obtiene un ancho de haz de $\pm 6^{\circ}$ para el haz primario y de $\pm 9^{\circ}$ y $\pm 15^{\circ}$ para el secundario en cada seno respectivo. Los ajustes para el haz primario en ambas señales son cercanos a la frecuencia primaria experimental $(200 \mathrm{kHz})$ y para el haz secundario, los ajustes son cercanos a una directividad de 
un seno de 168 y $147 \mathrm{kHz}$ respectivamente. Además, se puede observar una de las características de la generación paramétrica, que los lóbulos laterales en su mayoría son nulos o mínimos, como lo son para estos resultados.

\section{Atenuación}

Se varía la distancia $d$, entre emisor y receptor desde 0.50 a $2.50 \mathrm{~m}$, en pasos de 0.50 m. El ajuste de estos resultados es: $a \cdot d^{-b}$, donde el parámetro $b$ es la unidad o valores próximos a la misma para el haz primario (decaimiento hiperbólico, despreciando la absorción), y toma valores menores $( \pm 0.5)$ para el haz secundario [93].
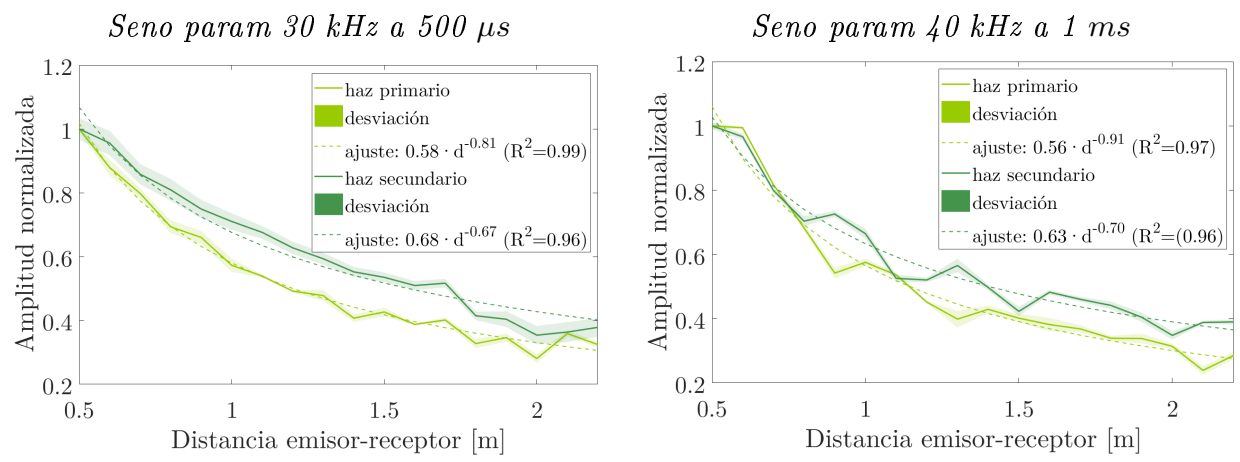

Figura 4.16: Atenuación de senos paramétricos. Valores de normalización para el haz primario de $12.2 m V_{\text {pico }}$ y para el secundario de $0.55 m V_{\text {pico }}$

En la Figura 4.16, se tiene para el seno de $30 \mathrm{kHz}$, que el parámetro $b$ corresponde a 0.81 y 0.67 para el haz primario y secundario respectivamente. Con el seno de $40 \mathrm{kHz}$, el valor de $b$ es de 0.91 en el haz primario y 0.70 en el secundario. La amplitud de presión del haz primario disminuye con el inverso de la distancia como es de esperar, y lo mismo ocurre para el haz secundario pero en menor proporción.

\section{Variación de voltaje}

Se emite con un valor de tensión desde los $200 \mathrm{mV}$ hasta $1 \mathrm{~V}$ en pasos de $100 \mathrm{mV}$, y se estudia la amplitud recibida. El ajuste se realiza según la expresión: $a \cdot V^{b}$, donde $V$ equivale al voltaje enviado.

En la Figura 4.17, se muestra que el exponente $b$ para el haz secundario es mucho más grande $(\gg 1)$ que el del haz primario $(\backsim 1)$, lo que evidencia el efecto no lineal (crecimiento exponencial). El valor del exponente para el haz primario es de 0.98 y 0.95 
Seno param $30 \mathrm{kHz}$ a $500 \mu \mathrm{s}$

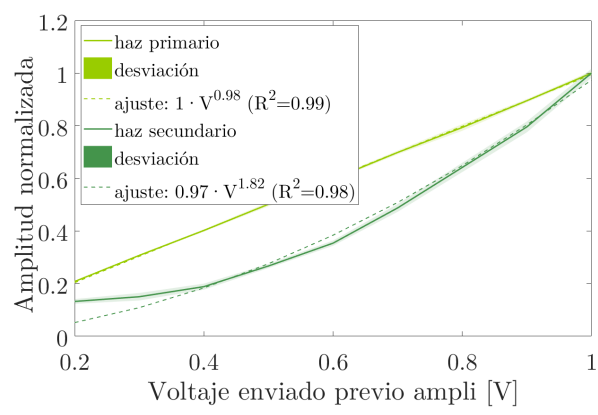

Seno param $40 \mathrm{kHz}$ a $1 \mathrm{~ms}$

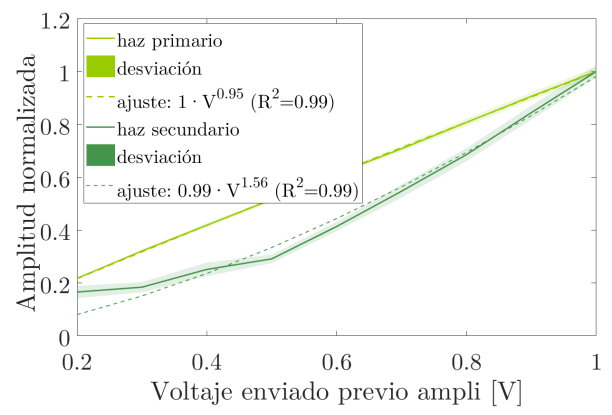

Figura 4.17: Variación de voltaje para senos paramétricos

respectivamente, y para el secundario es de 1.82 en el seno de $30 \mathrm{kHz}$ y 1.56 en el seno de $40 \mathrm{kHz}$. El exponente del haz primario es cercano a 1 como se espera en términos de linealidad. Al utilizar el método de correlación, se tiene para el paramétrico, que la señal recibida se correla con la segunda derivada de la envolvente al cuadrado de la señal enviada; por lo que debido al cuadrado, se espera en el ajuste un valor aproximado al doble respecto al valor $b$ del primario.

\subsubsection{Medidas con sweeps paramétricos}

En este último experimento, se estudia la generación de varios sweeps paramétricos en el dominio del tiempo partiendo de una $f_{p}$ de $200 \mathrm{kHz}$ modulada en amplitud por otras bajas frecuencias, obteniendo así la señal moduladora (sweep), y se emplea una $f_{s}$ de $20 \mathrm{MS} / \mathrm{s}$. Se presentan dos sweeps; el primero desde $4 \mathrm{kHz}$ hasta $40 \mathrm{kHz}$ a $1 \mathrm{~ms}$ de duración y el segundo desde 10 hasta $50 \mathrm{kHz}$ a $500 \mu \mathrm{s}$. Las medidas se realizan con una tensión de 1 Voltio a la entrada del amplificador.

Los análisis de los diferentes estudios paramétricos con estas señales, se obtuvieron tomando el valor de amplitud de correlación de la señal recibida con la señal del haz primario (alta frecuencia) y con la señal del haz secundario (baja frecuencia). 


\section{Directividad}

Sweep param 4 a $40 \mathrm{kHz}$ a $1 \mathrm{~ms}$

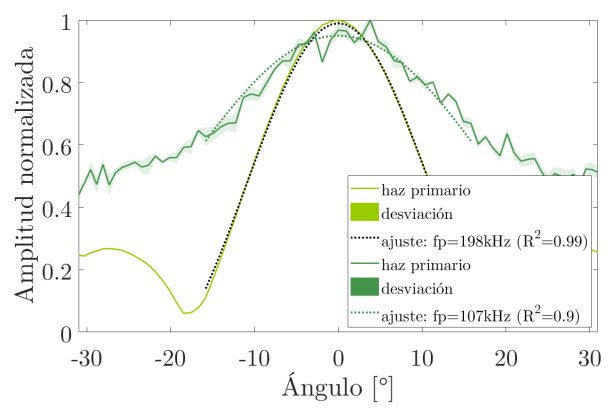

Sweep param 10 a $50 \mathrm{kHz} 500 \mu \mathrm{s}$

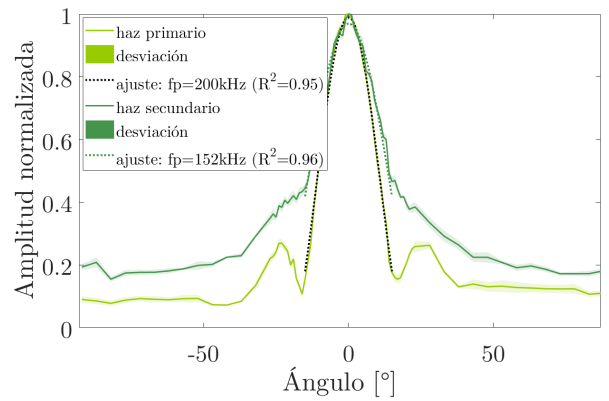

Figura 4.18: Directividad para sweeps paramétricos. Valores de normalización para el haz primario de $18 m V_{\text {pico }}$ y para el secundario de $0.22 m V_{\text {pico }}$

En la Figura 4.18, el ancho de haz del primario para ambos sweeps es de $\pm 8^{\circ}$ y de $\pm 12^{\circ}$ y $\pm 13^{\circ}$ en el secundario para cada sweep respectivamente. En general, las directividades para ambos haces son similares a pesar de que los contenidos espectrales son diferentes $(200 \mathrm{kHz}$ para el primario y bajas frecuencias hasta $50 \mathrm{kHz}$ para el secundario). Nuevamente se observa, que los lóbulos laterales para el haz secundario son nulos. Los ajustes para el haz primario en ambas señales son cercanos a la frecuencia primaria experimental $(200 \mathrm{kHz})$, y para el haz secundario, los ajustes son cercanos a una directividad de un seno lineal de 107 y $152 \mathrm{kHz}$ respectivamente.

A continuación, en la Figura 4.19, se presenta un ejemplo del nivel de presión sonora (SPL) para el sweep de 4 a $40 \mathrm{kHz}$ a $1000 \mu \mathrm{s}$.
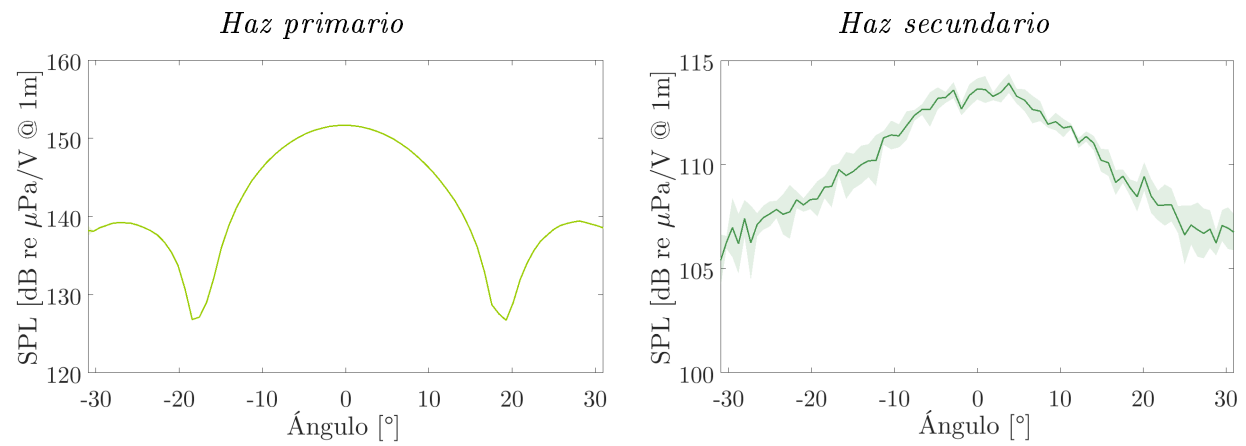

Figura 4.19: Directividad en SPL. Haz primario $152 \mathrm{~dB}$ y secundario de $114 \mathrm{~dB}$ 


\section{Atenuación}

La distancia entre los transductores varía desde los $15 \mathrm{~cm}$ hasta los $55 \mathrm{~cm}$ en pasos de $4 \mathrm{~cm}$ para el sweep de 4 a $40 \mathrm{kHz}$ y para el sweep de 10 a $50 \mathrm{kHz}$, la distancia entre transductores va desde los $0.50 \mathrm{~m}$ hasta los $2 \mathrm{~m}$ con pasos de $0.50 \mathrm{~m}$. el ajuste $a \cdot x^{-b}$ es el mismo que el empleado para los senos del apartado anterior. En la Figura 4.20, se tiene que el paramétro $b$ toma un valor de 0.94 y 0.88 para los haces primarios aproximándose a la unidad, mientras que para el haz secundario toma valores de 0.72 y 0.67 respectivamente para ambos sweeps, con una menor atenuación tal como se espera.

Sweep param 4 a $40 \mathrm{kHz}$ a $1 \mathrm{~ms}$

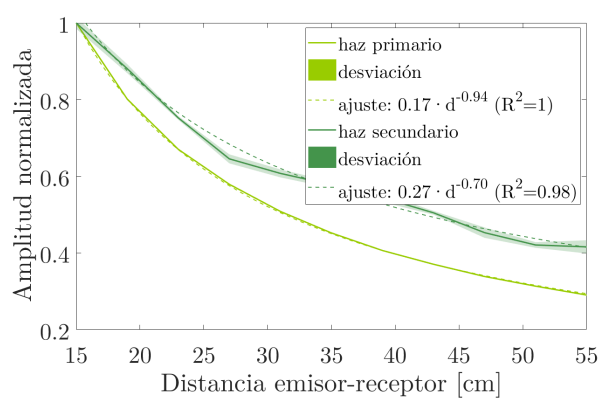

Sweep param 10 a $50 \mathrm{kHz} 500 \mu \mathrm{s}$

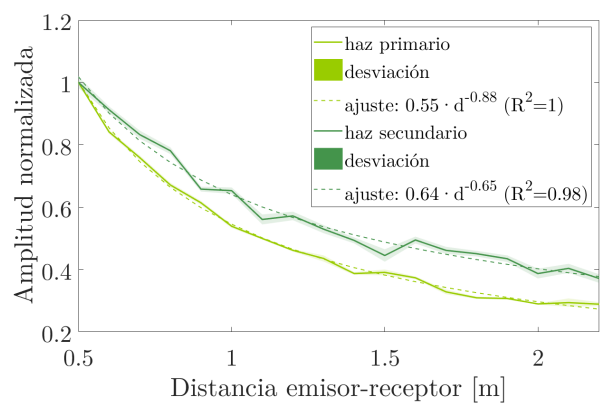

Figura 4.20: Atenuación para sweeps paramétricos. Valores de normalización para el haz primario de $18 m V_{\text {pico }}$ y para el secundario de $0.20 m V_{\text {pico }}$

\section{Variación de voltaje}

Utilizando el mismo ajuste del seno paramétrico $a \cdot V^{b}$. Se tiene en la Figura 4.21, que el ajuste para el haz primario en ambas señales sweeps paramétricos es muy próximo a la unidad, demostrando el efecto lineal, mientras que, para el haz secundario es de 1.84 y 1.64 respectivamente. Como la señal recibida se correla con la segunda derivada de la envolvente al cuadrado de la señal enviada; entonces, debido al cuadrado, se espera en el ajuste un valor aproximado al doble.

De acuerdo con los resultados obtenidos, tanto para los senos y sweeps paramétricos en este apartado, se alcanza una buena respuesta a la generación paramétrica. Sin embargo, detectar paramétricos a través de sweeps es mucho mejor por los resultados que estos ofrecen debido a que la detección por correlación con anchos de banda frecuenciales amplios permiten un pico de correlación estrecho, como se analizó en el Capítulo 3. 
Sweep param 4 a $40 \mathrm{kHz}$ a $1 \mathrm{~ms}$

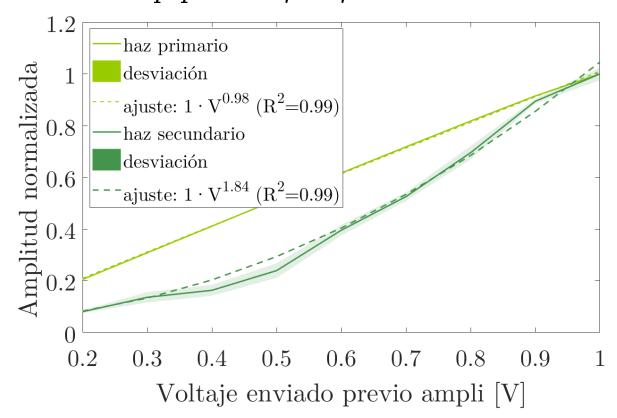

Sweep param 10 a $50 \mathrm{kHz} 500 \mu \mathrm{s}$

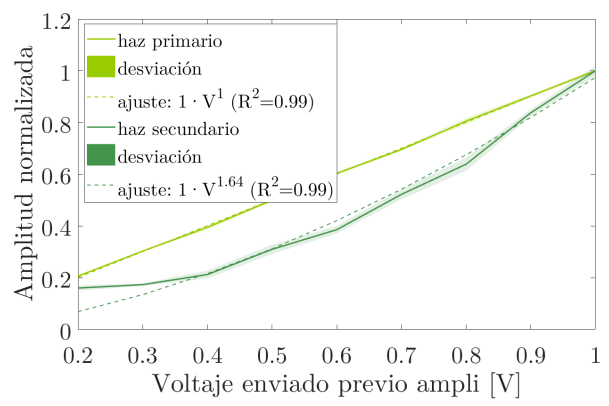

Figura 4.21: Variación de voltaje para senos paramétricos

Estos experimentos realizados en el laboratorio, que corresponden a los estudios de: patrón de directividad, dependencia de la amplitud del haz secundario con el voltaje aplicado para alimentar el transductor (variación de voltaje) y la distancia (atenuación) demuestran que la técnica de generación paramétrica es una herramienta que podría utilizarse para generar señales aplicadas a las comunicaciones acústicas, sea bien utilizando un conjunto de arrays, o sonares paramétricos. 



\section{Capítulo 5 \\ Aplicación a las comunicaciones acústicas}

Las comunicaciones acústica submarinas se están convirtiendo en un campo creciente en la investigación acústica, ya que permiten estudiar los procesos de interacción de una señal en el agua y aprovechar sus características en diversas aplicaciones. Sin embargo, este tipo de comunicaciones presentan dificultades, debido a la interferencia de la señal por las múltiples reflexiones presentes en el medio, lo que imposibilita una buena recepción de la señal acústica propagada.

Para lograr comunicaciones estables, es necesario el estudio y aplicación de distintas técnicas (modulaciones) utilizadas a lo largo de las últimas décadas que permitan comunicaciones con una baja tasa de error $\left(\mathrm{BER}^{1}\right)$.

Desde los estudios de la teoría relacionada con el efecto paramétrico derivada por Westervelt, y presentados en esta tesis en el Capítulo 1, se vienen desarrollando sistemas paramétricos en los campos de la detección en el fondo marino y comunicaciones submarinas. Este capítulo, tiene como interés propio, presentar los resultados obtenidos en las medidas experimentales realizadas en aguas poco profundas. Para ello, se tiene en el apartado 5.1, una breve reseña de los antecedentes en las comunicaciones, explicado con mayor profundidad en el Capítulo 2. Posteriormente, en el apartado 5.2, se presentan los resultados de las modulaciones medidas (senos y sweeps) con una cadena de bits y se estudia la capacidad de detección por medio de la correlación cruzada.

\footnotetext{
${ }^{1}$ BER: Bit error
} 


\subsection{Contexto/antecedentes}

Como se comentó en el Capítulo 1, el estudio de las fuentes acústica paramétricas fue concebido por Westervelt cuya teoría se basa en el scattering of sound by sound (dispersión de sonido por sonido). En sus estudios concluyó, que este tipo de fuentes paramétricas ofrecen un haz estrecho en bajas frecuencias con lóbulos laterales mínimos utilizando un conjunto de transductores (array) de pequeñas dimensiones. Sin embargo, el principal inconveniente de la utilización de esta técnica es, la baja eficiencia de conversión de las señales primarias a las señales secundarias que es de aproximadamente $20 \mathrm{~dB}$ menor que, el nivel de fuente de la señal primaria lo que dificulta el desarrollo y posteriores aplicaciones. A pesar de esto, se vienen diseñando diferentes sondas comerciales como las presentadas en la Tabla 5.1 con esta técnica, y utilizadas para la penetración en los sedimentos del fondo marino y detección de objetos entrerrados.

De las ecuaciones derivadas por Westervelt y Berktay, se tiene que, si se aumenta el nivel de la fuente primaria y secundaria, se puede lograr una contribución a mejorar la eficiencia de conversión. Sin embargo, el nivel de la fuente primaria no se puede aumentar indefinidamente debido al fenómeno de saturación acústica. Así mismo, el aumento de la fuente secundaria está limitada; al aumentar la frecuencia secundaria habrá una mayor atenuación, y las profundidades de penetración serán mas bajas. Así pues, para mejorar la eficiencia de conversión se está investigando en la optimización del material del transductor y su diseño, por ejemplo, algunos investigadores fabricaron transductores ultrasónicos $\left(\mathrm{CMUT}^{2}\right)$ con cavidades selladas al vacío, estos dispositivos produjeron haces de alta intensidad a $50 \mathrm{kHz}$ que producían haces estrechos de $8.7^{\circ}$ en $5 \mathrm{kHz}$ a $3 \mathrm{~m}$ [94]. Otros estudios se pueden profundizar en $[95,96]$.

Las comunicaciones acústicas submarinas de alto rendimiento requieren altos anchos de banda para altas velocidades de datos y un haz estrecho para un menor efecto de las reflexiones en el trayecto multicamino. Todos esto requisitos se pueden llegar a cumplir si se emplean arrays acústicos paramétricos aplicando las diferentes técnicas de modulación existentes, y así lograr un buen rendimiento del sistema. Una serie de publicaciones que tratan de comunicaciones paramétricas fueron presentados en el Capítulo 2, Tabla 2.2. En ellas, se alcanzaron rangos de decenas de kilómetros y velocidades de datos de 1,2 y $3 \mathrm{kbit} / \mathrm{s}$. Además, la ventaja de obtener un ancho de haz estrecho (directivo) puede mejorar la capacidad de información de una red y enviar mensajes a los nodos deseados sin ser interceptados. Por lo tanto, esto es potencialmente útil en el ámbito militar donde es importante que la señal de transmisión de información viaje encubierta [97].

\footnotetext{
${ }^{2}$ Capacitive Micromachined Ultrasonic Transducers
} 
De igual forma, el estudio de la generación paramétrica se ha utilizado en proyectos tales como, el MAST [62] financiados por la UE, en el que se estudió la comunicación submarina de largo alcance en canales de aguas poco profundas mediante investigaciones de perfiles del fondo y sub-fondo marino.

\begin{tabular}{|c|c|c|c|c|c|c|c|}
\hline 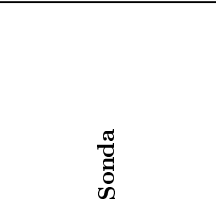 & 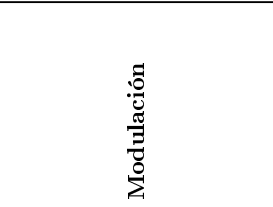 & $\frac{\bar{N}}{\underbrace{2}}$ & $\frac{\underset{ \pm}{\mathbb{N}}}{\underbrace{\infty}}$ & 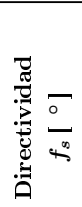 & 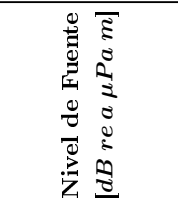 & 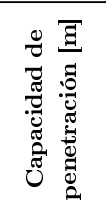 & 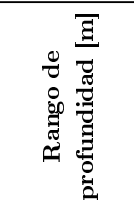 \\
\hline SES 2000 smart [98] & CW, Ricker & $90-110$ & $5-15$ & $\pm 2.5^{\circ}$ & 236 & hasta 20 & $<0.5-100$ \\
\hline Topas P18 [99] & Chirp, Ricker & $15-21$ & $0.5-6$ & $\pm 4.5^{\circ}$ & $4 \mathrm{kHz}=\sim 209$ & $>200$ & $<20-11000$ \\
\hline BII - 7543 [100] & Ricker, PSK, FSK, Pulso & $180-210$ & $2-20$ & $\pm 8^{\circ}$ & $10 \mathrm{kHz}=>199$ & $>20$ & Máx. 300 \\
\hline SeaKing SBP [101] & Pulso, Seno & 200 & 20 & $\pm 4^{\circ}$ & - & - & Hasta 400 \\
\hline
\end{tabular}

Tabla 5.1: Sondas paramétricas comerciales

\subsection{Capacidad de detección}

A continuación, se presentan los resultados obtenidos de realizar diferentes medidas experimentales de modulaciones en paramétrico [102], emitiendo y recibiendo con los transductores enfrentados a una distancia de $30 \mathrm{~cm}$ medidos en piscina de laboratorio, con el fin de analizar la capacidad de detección de cada una de estas, con la técnica de la correlación cruzada. Se emite en el agua la siguiente cadena de bits [01101111 01101011] que corresponde a la palabra: ok. La frecuencia portadora en cada una de las medidas fue de $200 \mathrm{kHz}$ y se utilizó frecuencia de muestreo de $20 \mathrm{MHz}$.

Una vez se procesan las señales, se obtiene el bit error (BER) de cada una de ellas con la siguiente expresión $B E R(\%)=($ bits no detectados / bits totales $) \cdot 100$.

Como se comentó en capítulos anteriores, las técnicas de modulaciones empleadas consisten en la concatenación de señales tipo seno y, además por extensión, se estudian las señales tipo sweeps (señales de banda ancha). En los siguientes apartados se comentarán las características de estos conjuntos de señales. 


\subsubsection{Modulaciones con senos y sweeps paramétricos}

\section{ASK (OOK)}

En esta modulación no se detectan los bits 0, por ser todos de amplitud cero y no compatible con la correlación, dado que es una modulación de encendido y apagado, tal como se explicó en el Capítulo 2. Así que, para este caso no se podría hablar de $\mathrm{BER}$, sin embargo, se obtienen los resultados de correlación para el bit 1 .

El subapartado 5.2.2, Figura 5.1, presenta un ejemplo de ello. Se observa una clara detección por correlación del bit 1, aun así, sus picos de correlación no son estrechos, por ser señales de banda estrecha.

\section{FSK}

Paramétricamente esta modulación se puede obtener utilizando dos señales moduladoras $f_{1}$ y $f_{2}$ cuyas frecuencias sean la mitad de las frecuencias asociadas a cada uno de los bits que se quieren recibir $\left(f_{1}=f_{\text {bit } 1} / 2\right.$ para el bit 1, y $f_{0}=f_{\text {bit } 0} / 2$ para el bit 0), la alternancia de una frecuencia a la otra, representa el cambio de bit correspondiente enlazados hasta reproducir el código binario deseado. Mediante esta técnica no lineal, modulando una portadora con una FSK se obtiene otra FSK del doble de frecuencia ( como se vio en el Capítulo 1, ecuación (1.111)), que corresponde a la señal paramétrica esperada.

La Figura 5.2 del subapartado 5.2.2, muestra un ejemplo de señal enviada y recibida para el bit 1 y 0 , que corresponde a la señal medida con $f_{1} \quad 20 \mathrm{kHz}$ y $f_{2} \quad 40$ $\mathrm{kHz}$ esperadas paramétricamente a $500 \mu \mathrm{s}$, y una frecuencia portadora de $200 \mathrm{kHz}$. En ella, se concluye lo comentado en el capítulo Capítulo 3, al ser una modulación basada en senos (tonos), se observa que la detección por correlación no presenta un pico estrecho, sin embargo, hay una tendencia clara en la detección para cada bit enviado.

\section{PSK}

Esta modulación se obtiene con señales moduladoras a la mitad que se desean obtener paramétricamente. Su generación paramétrica consiste en utilizar una frecuencia concreta para el bit 1 , y para el bit 0 , la misma frecuencia mas un desfase de $180^{\circ}$. Con ello, modulando una portadora con cada uno de estos bits, se obtiene la cadena de bits enlazados y esperados al doble (1.111) de la moduladora (paramétrica) tal como 
lo muestra la Tabla 5.3, donde se presentan todas las señales medidas y su respectivo BER.

El subapartado 5.2.2, Figura 5.3, muestra un ejemplo para una de las señales PSK medidas en piscina, con una $f_{p}$ de $200 \mathrm{kHz}$ y frecuencia paramétrica de $50 \mathrm{kHz}$ a 300 $\mu s$.

\section{Sweeps}

En esta tesis, se utilizan los barridos senoidales como señal de modulación para mejorar el comportamiento de las modulaciones anteriores; donde uno es ascendente en frecuencia con el tiempo (bit 1) y el otro descendente (bit 0). Para obtenerlos paramétricamente se debe tener en cuenta que la señal moduladora será la mitad de la señal paramétrica esperada, tal como se ha comentado en las anteriores modulaciones, y enlazando cada uno de ellos se genera la cadena de bits deseada.

En el siguiente, apartado la Figura 5.4, muestra un ejemplo para el sweep medido esperado entre los 10 y $50 \mathrm{kHz}$ correspondiente al bit 1 , y de forma descendente, 50 a $10 \mathrm{kHz}$ para el bit 0 a $1000 \mu s$ de duración por bit.

En general, empleando este conjunto de señales sweeps paramétricos, se obtiene una mejora en la detección de los bits, independiente del tamaño de los tiempos.

\subsubsection{Detección de los bits}

En la Figura 5.1, Figura 5.2, Figura 5.3 y Figura 5.4. Se presentan por separado las señales emitidas del bit 1 y el bit 0 , para una mejor visualización. Sin embargo, en la práctica estas señales se envían concatenadas según la cadena de bits definida en el apartado 5.2, que por comodidad se vuelve a escribir como [01101111 01101011 . Posteriormente, y haciendo referencia al esquema de la Figura 3.10, se extrae de la señal enviada (cadena de bits) la segunda derivada de la envolvente al cuadrado para ambos bits (señal paramétrica esperada), tal como se observa en las figuras. Luego, la señal recibida en cadena de bits, se filtra alrededor de la baja frecuencia (señal en ocre). Con esto, se correla la señal recibida filtrada con la señal esperada del bit 1; obteniendo así en principio una mayor amplitud para las diferentes posiciones del bit 1 (que en adelante se llamarán: bit verdadero), y una detección nula en principio para el bit 0 (amplitudes que se llamarán: bit falso). Si por el contrario, cuando se correla la recibida filtrada con la esperada del bit 0 , se obtendrá una mayor amplitud para las diferentes posiciones del bit 0 (bit verdadero) y una amplitud menor o nula para el bit 1 (bit falso). Se obtiene así, la correación cruzada tanto para el bit 1 como para el 0 junto con las amplitudes de correlación que se comentarán a continuación. 
Siendo la distancia $d$, entre el emisor y receptor de $30 \mathrm{~cm}$, una velocidad del sonido $v$, de aproximadamente $1480 \mathrm{~m} / \mathrm{s}$, y siguiendo la expresión del tiempo de vuelo $t=d / v$, el primer bit que se espera detectar es a los $0.2 \mathrm{~ms}$, y cada uno de los siguientes se esperan en el tiempo que se midió cada señal, es decir: 250, 500, 300 y $1000 \mu s$.

En todas las correlaciones, la línea discontinua representa la posción en tiempo de los bits 1, y la continua representa la posición esperada de los bits 0. En general, se observa que se tiene una buena detección temporal de cada bit en cada modulación.

Respecto a la Figura 5.1 ASK, los picos de correlación son bastante anchos entre si, casi que del orden de duración de cada bit, básicamente esto se debe, a que este tipo de señales son en esencia tonos puros. Sin embargo, las amplitudes de detección por correlación son claras entre el bit 1 de $0.001 \cdot 10^{-4}$ y el bit falso de $0.005 \cdot 10^{-4} ; 4$ órdenes de magnitud entre ellas.

En la Figura 5.2 FSK, sucede algo muy parecido a la ASK. En definitiva, las correlaciones con señales de banda estrecha se caracterizan por no ser demasiado eficientes en su detección. Por su parte, el promedio de las amplitudes de los bits 1 y 0 detectados correctamente es de $0.024 \cdot 10^{-4}$ y $0.061 \cdot 10^{-4}$, y de los bits falsos de $0.007 \cdot 10^{-4}$ y $0.014 \cdot 10^{-4}$. El conciente entre los bits detectados respecto de los falsos, dará una estimación del bit error, que se presenta en la siguiente sección.

La Figura 5.3 PSK, muestra que la señal recibida por correlación para ambos bits (1 y 0 ) es la misma, dado que la señal esperada con la que se correla es igual. Concerniente a la detección, a simple vista no hay una diferenciación de las amplitudes entre los bits que en principio deben detectarse con respecto a los falsos. Sin embargo, una vez se detecta un bit, la duración de los demás se espera en unos tiempos dados y fijos; por ello, la detección es relativamente buena a pesar de que a simple vista no lo parezca. El promedio de las amplitudes para los bits verdaderos (1 y 0 respectivamente) es de $0.14 \cdot 10^{-4}$ y $0.13 \cdot 10^{-4}$ mientras que para los falsos bits es de $0.13 \cdot 10^{-4}$ y $0.14 \cdot 10^{-4}$. Estos resultados justifican la poco claridad que existe al momento de discernir las amplitudes de unos bit con respecto a otros.

En este estudio en cuestión, este tipo de modulación no fue robusta para las comunicaciones paramétricas, aunque si que lo es para otro tipo de comunicaciones.

En la Figura 5.4 Sweep paramétrico, se obtuvieron picos de correlación estrechos a diferencia de las demás modulaciones, y los bits son mucho más fáciles de discernir. La detección por correlación para el bit 1 y 0 es de $0.19 \cdot 10^{-4}$ y $0.073 \cdot 10^{-4}$, y para los bits falsos 1 y 0 , corresponde a $0.018 \cdot 10^{-4}$ y $0.025 \cdot 10^{-4}$ respectivamente.

De todas las modulaciones estudiadas se tiene que los picos de correlación se detectan muy cercanos respecto de los tiempos esperados, teniendo en cuenta el tiempo de vuelo 
inicial donde se espera ser recibida dicha señal. El comportamiento de la correlación y el ancho de los picos resultantes tiene consecuencias tanto en la detección como en la discriminación de bits.

De entre todas las anteriores modulaciones (senos: ASK, FSK, PSK y sweep), se observa que, en la detección por correlación con sweeps [103], se presentan picos estrechos y claros, tal como se vio en el Capítulo 3, debido a que son señales de banda ancha. El máximo de correlación se comporta de forma más estrecha, la señal se amortiza mucho más rápido, por lo tanto, se tiene una amplitud de correlación y un ancho de la misma mucho más definido.

Emitida bit 1

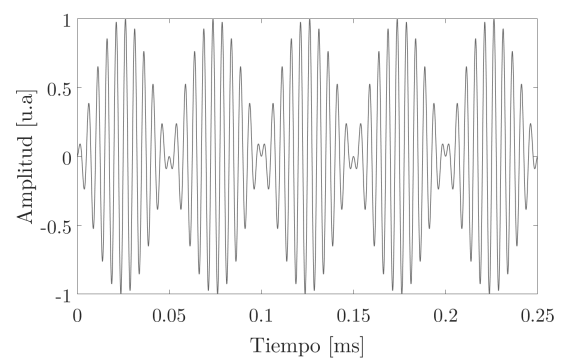

Recibida bit 1

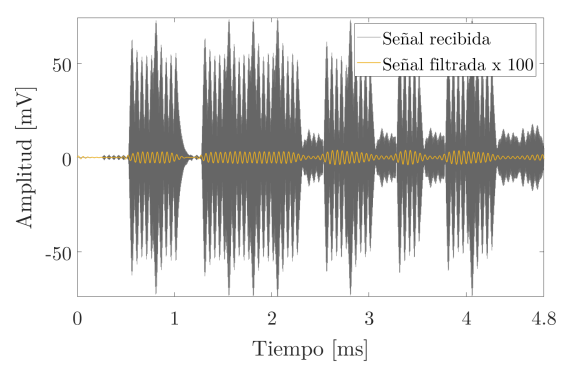

Esperada bit 1

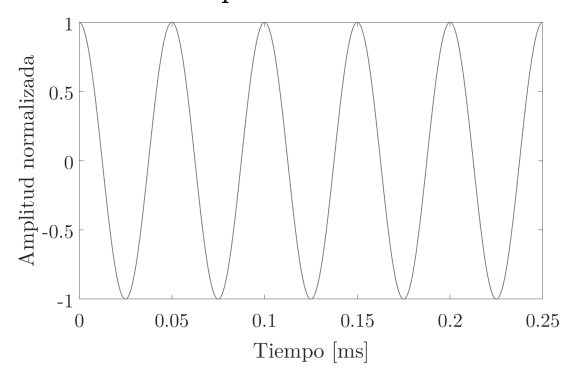

Correlación bit 1

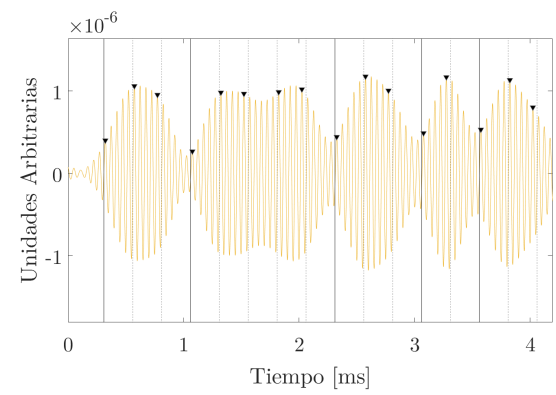

Figura 5.1: Análisis detección por correlación para la cadena de bits [01101111 01101011] en modulación ASK, bit ${ }_{1}=10 \mathrm{kHz}$ a $250 \mu s$ por cada bit, $f_{p}$ de $200 \mathrm{kHz}$ 
Emitida bit 1

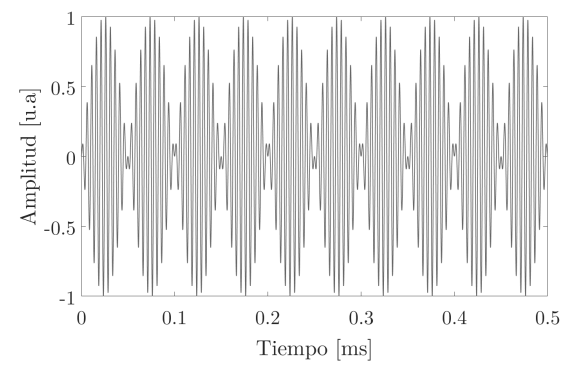

Esperada bit 1

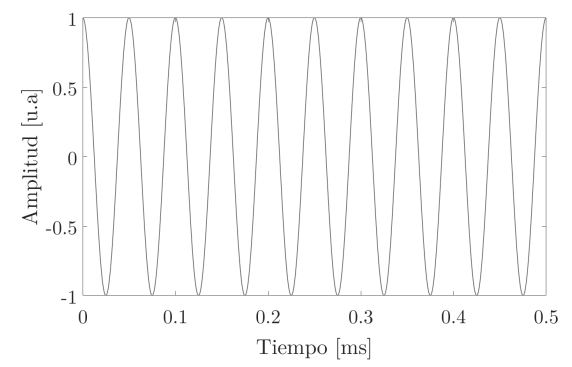

Recibida bit 1

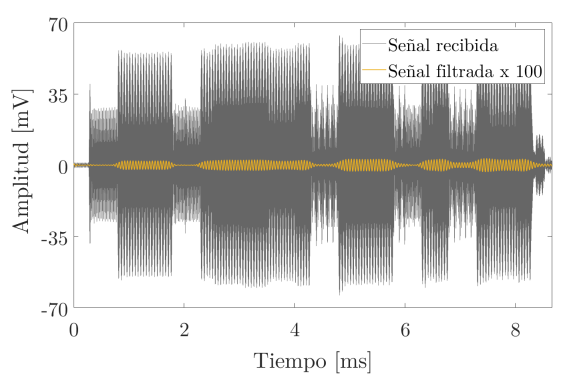

XCorr bit 1

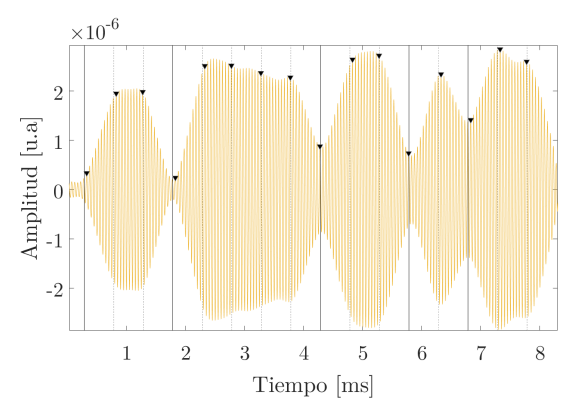

Emitida bit 0

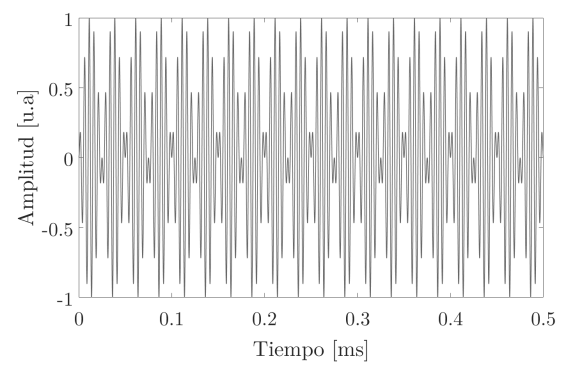

Esperada bit 0

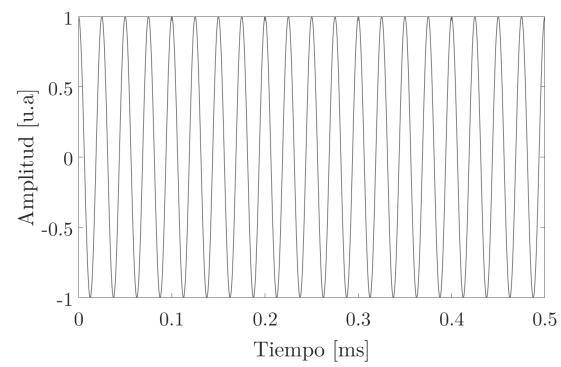

Recibida bit 0

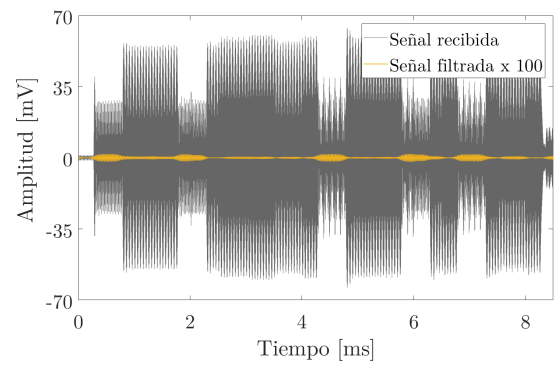

$X$ Corr bit 0

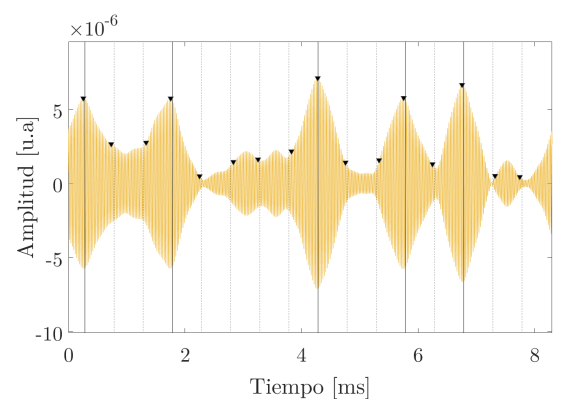

Figura 5.2: Análisis detección por correlación para la cadena de bits [01101111 01101011$]$ con modulación FSK, bit $1 \quad 20 \mathrm{kHz}$ y bit $t_{0} \quad 40 \mathrm{kHz}$ a $500 \mu s$ por cada bit, $f_{p}$ de $200 \mathrm{kHz}$ 
Emitida bit 1

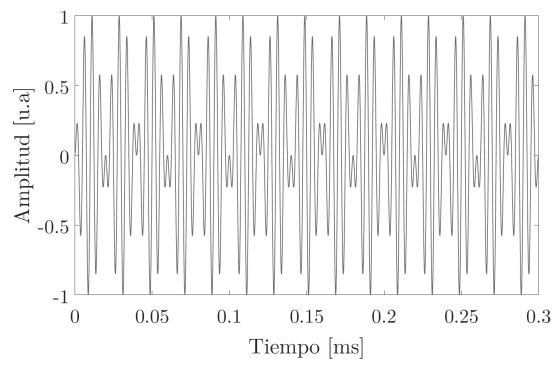

Esperada bit 1

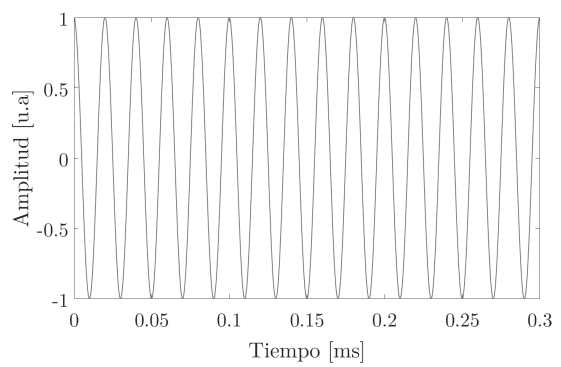

Recibida bit 1

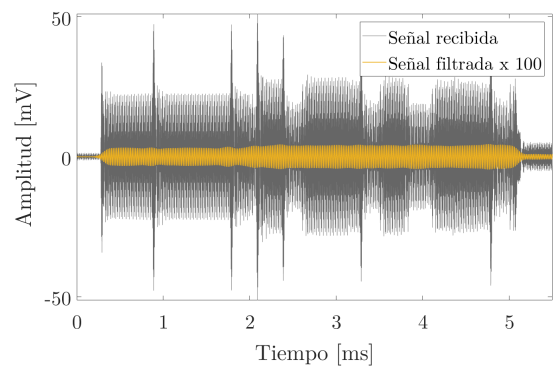

Emitida bit 0

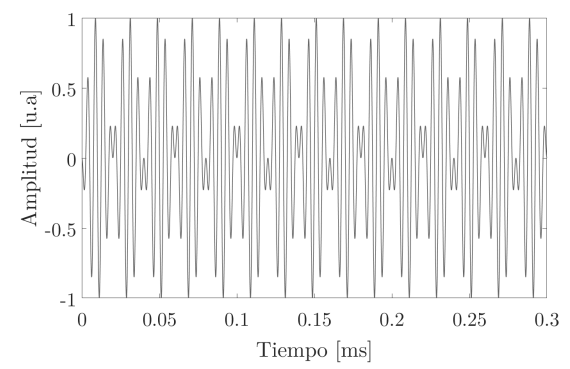

Esperada bit 0

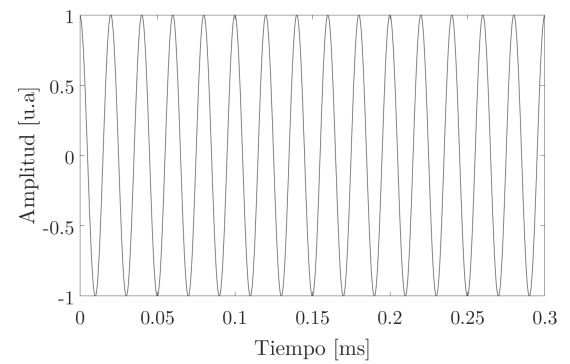

Recibida bit 0

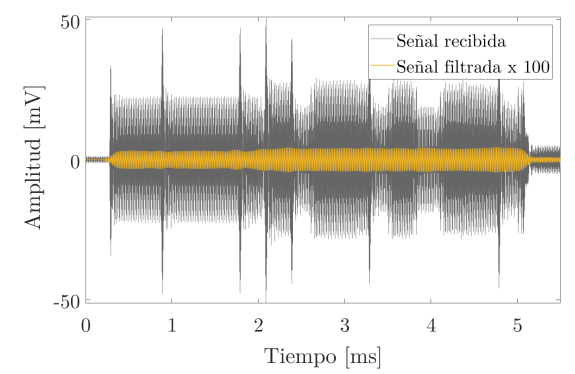

XCorr bit 1

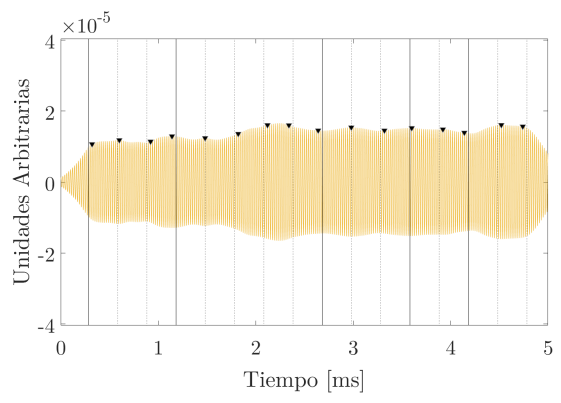

Figura 5.3: Análisis detección por correlación para la cadena de bits [01101111 01101011] en modulación PSK con $f_{\text {param }}$ de $50 \mathrm{kHz}$ a $300 \mu s$ por cada bit, $f_{p}$ de $200 \mathrm{kHz}$ 
Emitida bit 1

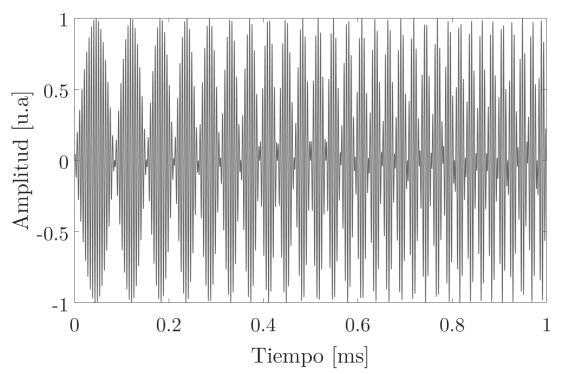

Esperada bit 1

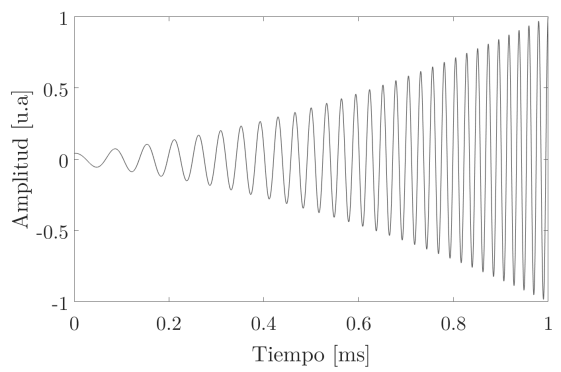

Emitida bit 0

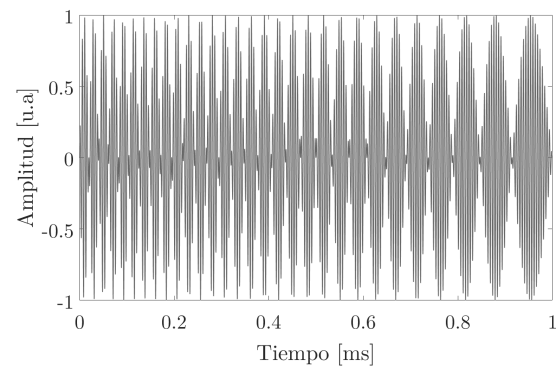

Esperada bit 0

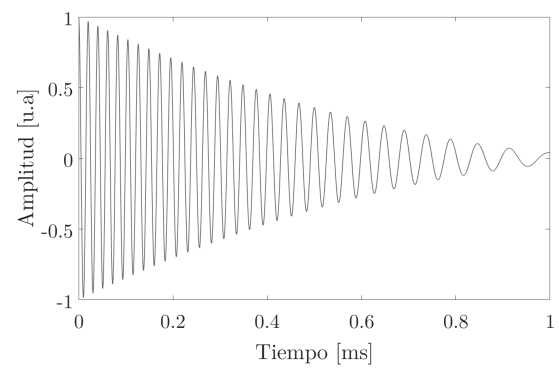

Recibida

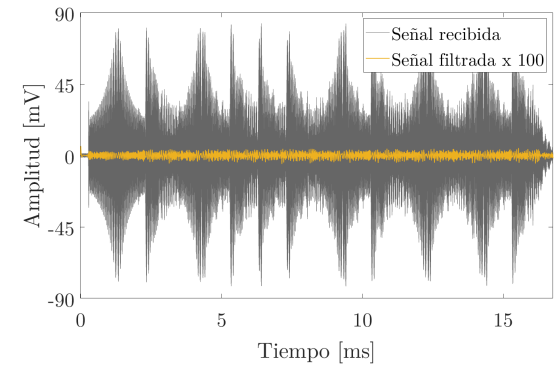

XCorr bit 1

XCorr bit 0
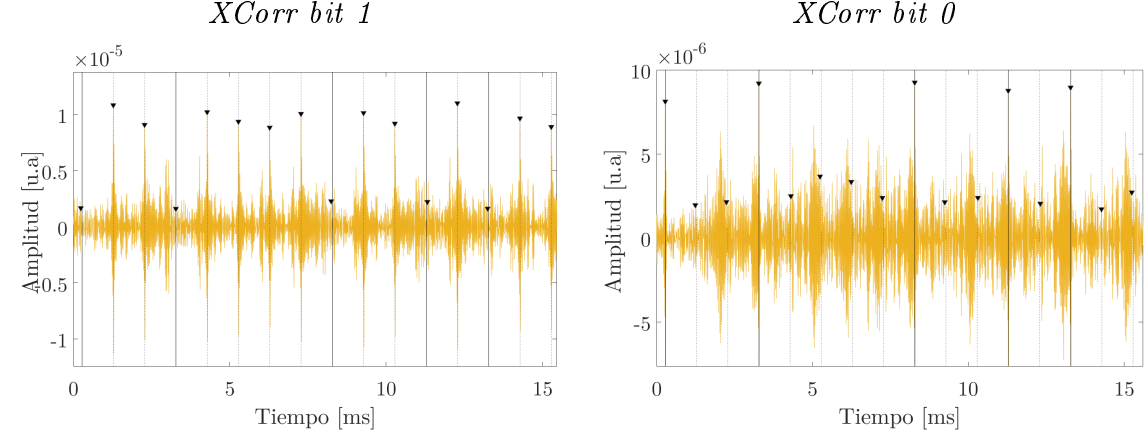

Figura 5.4: Análisis etección por correlación para la cadena de bits [01101111 01101011] con Sweep, bit $t_{1}=10$ a $50 \mathrm{kHz}$, bit $_{0}=50$ a $10 \mathrm{kHz}$ a $1000 \mu \mathrm{s}$ por cada bit, $f_{p}$ de $200 \mathrm{kHz}$ 


\subsubsection{Bit Error (BER)}

\section{FSK}

En la Tabla 5.2, se presentan las medidas realizadas para este tipo de señales esperadas paramétricamente, junto con el bit error. Se observa cierta tendencia de que, cuanto mayor es el tiempo de duración de cada bit, existe un menor error de los bits detectados, inferior al $20 \%$. Sin embargo, para el primer grupo de señales enviadas $(10,30 \mathrm{kHz})$ y $(10,40 \mathrm{kHz})$ se obtuvo un BER superior al $50 \%$. Respecto al valor de la desviación del BER, este no supera el \pm 7 .

\begin{tabular}{|c|c|c|c|c|c|c|c|}
\hline \multicolumn{2}{|c|}{ Frecuencias $[\mathrm{kHz}]$} & \multirow{2}{*}{ Duración $[\mu s]$} & \multirow{2}{*}{ BER [\%] } & \multicolumn{2}{|c|}{ Frecuencias [kHz] } & \multirow{2}{*}{ Duración $[\mu s]$} & \multirow{2}{*}{ BER [\%] } \\
\hline$f_{1}$ & $f_{2}$ & & & $f_{1}$ & $f_{2}$ & & \\
\hline 10 & 30 & 500 & $59 \pm 3$ & & & 250 & $25 \pm 0$ \\
\hline 10 & 40 & 1000 & $64 \pm 5$ & 30 & 40 & 500 & $19 \pm 0$ \\
\hline \multirow{3}{*}{20} & \multirow{3}{*}{30} & 250 & $20 \pm 7$ & & & 1000 & $20 \pm 7$ \\
\hline & & 500 & $11 \pm 3$ & \multirow{5}{*}{30} & \multirow{5}{*}{50} & 100 & $66 \pm 3$ \\
\hline & & 1000 & $11 \pm 5$ & & & 167 & $45 \pm 5$ \\
\hline \multirow{3}{*}{20} & \multirow{3}{*}{40} & 250 & $46 \pm 3$ & & & 250 & $69 \pm 0$ \\
\hline & & 500 & $15 \pm 7$ & & & 500 & $15 \pm 7$ \\
\hline & & 1000 & $8 \pm 3$ & & & 1000 & $10 \pm 3$ \\
\hline \multirow{3}{*}{20} & \multirow{3}{*}{50} & 250 & $6 \pm 3$ & & & 50 & $69 \pm 0$ \\
\hline & & 500 & $0 \pm 0$ & & & 100 & $53 \pm 3$ \\
\hline & & 1000 & $0 \pm 0$ & 40 & 50 & 125 & $56 \pm 4$ \\
\hline \multirow{2}{*}{30} & \multirow{2}{*}{40} & 100 & $20 \pm 7$ & & & 250 & $50 \pm 0$ \\
\hline & & 167 & $36 \pm 5$ & & & 1000 & $9 \pm 7$ \\
\hline
\end{tabular}

Tabla 5.2: BER de la modulación FSK para un conjunto de señales medidas

\section{PSK}

En la Tabla 5.3, se observa que, entre las señales y los tiempos de duración, no existe una tendencia clara al momento de detectar el BER. Se tiene un BER superior al $50 \%$, en las señales de $30 \mathrm{kHz}$ a 67,167 y $333 \mu \mathrm{s}, 35 \mathrm{kHz}$ a 57 y $429 \mu s, 40 \mathrm{kHz}$ a 50 $\mu s$, y $50 \mathrm{kHz}$ a 40 y $300 \mu s$. 


\begin{tabular}{|c|c|c|c|c|c|}
\hline Frecuencias [kHz] & Duración $[\mu \mathrm{s}]$ & BER [\%] & Frecuencias $[\mathrm{kHz}]$ & Duración [ $\mu \mathrm{s}]$ & BER [\%] \\
\hline \multirow{2}{*}{10} & 500 & $25 \pm 9$ & \multirow{5}{*}{35} & 57 & $69 \pm 0$ \\
\hline & 1000 & $28 \pm 10$ & & 86 & $16 \pm 8$ \\
\hline \multirow{4}{*}{15} & 200 & $41 \pm 11$ & & 143 & $10 \pm 4$ \\
\hline & 333 & $63 \pm 5$ & & 286 & $11 \pm 7$ \\
\hline & 667 & $69 \pm 0$ & & 429 & $66 \pm 3$ \\
\hline & 1000 & $9 \pm 5$ & \multirow{5}{*}{40} & 50 & $64 \pm 5$ \\
\hline \multirow{3}{*}{20} & 250 & $29 \pm 10$ & & 75 & $14 \pm 11$ \\
\hline & 500 & $11 \pm 7$ & & 125 & $14 \pm 9$ \\
\hline & 750 & $10 \pm 5$ & & 250 & $50 \pm 10$ \\
\hline \multirow{5}{*}{25} & 80 & $0.6 \pm 2$ & & 375 & $30 \pm 12$ \\
\hline & 120 & $23 \pm 9$ & \multirow{5}{*}{45} & 44 & $34 \pm 16$ \\
\hline & 200 & $29 \pm 14$ & & 67 & $8 \pm 8$ \\
\hline & 400 & $24 \pm 10$ & & 111 & $8 \pm 5$ \\
\hline & 600 & $34 \pm 10$ & & 222 & $13 \pm 10$ \\
\hline \multirow{6}{*}{30} & 67 & $69 \pm 0$ & & 333 & $0 \pm 0$ \\
\hline & 100 & $23 \pm 13$ & \multirow{5}{*}{50} & 40 & $62 \pm 6$ \\
\hline & 167 & $68 \pm 2$ & & 60 & $15 \pm 14$ \\
\hline & 333 & $69 \pm 0$ & & 100 & $0 \pm 0$ \\
\hline & \multirow[t]{2}{*}{500} & $35 \pm 11$ & & 200 & $47 \pm 12$ \\
\hline & & & & 300 & $52 \pm 12$ \\
\hline
\end{tabular}

Tabla 5.3: BER de la modulación PSK para un conjunto de señales medidas

\section{Sweep}

Para este análisis, se midieron un conjunto de señales tipo sweeps con diferentes tiempos y anchos de banda. En la Tabla 5.4, se muestran todas ellas junto con el BER.

Se observa, que la máxima tasa de error es para el sweep desde los 40 a $50 \mathrm{kHz}$ con $90 \mu s$ con un BER del $58 \%$. Es probable que esto se deba, que al ser un tiempo tan corto para cada bit, no alcancen a detectarse adecuadamente porque se mezclan con las reflexiones provenientes del fondo y superficie de la piscina. Para el resto de los sweeps, el BER ronda alrededor de una media del $7 \%$. 


\begin{tabular}{|c|c|c|c|c|c|}
\hline Frecuencias [kHz] & Duración $[\mu \mathrm{s}]$ & BER [\%] & Frecuencias [kHz] & Duración $[\mu \mathrm{s}]$ & BER [\%] \\
\hline \multirow{2}{*}{5 a 15} & 600 & $14 \pm 3$ & \multirow{3}{*}{10 a 40} & 160 & $0 \pm 0$ \\
\hline & 1000 & $0 \pm 0$ & & 320 & $0 \pm 0$ \\
\hline \multirow{3}{*}{5 a 25} & 267 & $1 \pm 3$ & & 480 & $0 \pm 0$ \\
\hline & 400 & $0 \pm 0$ & \multirow{4}{*}{10 a 50} & 134 & $1 \pm 3$ \\
\hline & 533 & $0 \pm 0$ & & 267 & $0 \pm 0$ \\
\hline \multirow{3}{*}{5 a 35} & 200 & $1 \pm 3$ & & 400 & $1 \pm 3$ \\
\hline & 400 & $0 \pm 0$ & & 1000 & $0 \pm 0$ \\
\hline & 600 & $0 \pm 0$ & \multirow{3}{*}{20 a 30} & 160 & $15 \pm 7$ \\
\hline \multirow{4}{*}{5 a 45} & 160 & $6 \pm 8$ & & 320 & $0 \pm 0$ \\
\hline & 320 & $1 \pm 3$ & & 480 & $0 \pm 0$ \\
\hline & 480 & $0 \pm 0$ & \multirow{3}{*}{20 a 40} & 134 & $0 \pm 0$ \\
\hline & 147 & $8 \pm 5$ & & 267 & $0 \pm 0$ \\
\hline \multirow{2}{*}{5 a 50} & 291 & $3 \pm 3$ & & 402 & $0 \pm 0$ \\
\hline & 438 & $0 \pm 0$ & \multirow{3}{*}{20 a 50} & 115 & $13 \pm 8$ \\
\hline \multirow{2}{*}{4 a 40} & 500 & $0 \pm 0$ & & 229 & $0 \pm 0$ \\
\hline & 1000 & $0 \pm 0$ & & 343 & $0 \pm 0$ \\
\hline \multirow{3}{*}{10 a 20} & 267 & $5 \pm 3$ & \multirow{3}{*}{30 a 40} & 115 & $15 \pm 14$ \\
\hline & 534 & $0 \pm 0$ & & 229 & $6 \pm 0$ \\
\hline & 801 & $0 \pm 0$ & & 343 & $0 \pm 0$ \\
\hline \multirow{6}{*}{10 a 30} & 160 & $4 \pm 6$ & \multirow{3}{*}{30 a 50} & 100 & $18 \pm 8$ \\
\hline & 200 & $0 \pm 0$ & & 200 & $0 \pm 0$ \\
\hline & 320 & $10 \pm 7$ & & 600 & $0 \pm 0$ \\
\hline & 400 & $0 \pm 0$ & \multirow{3}{*}{40 a 50} & 90 & $58 \pm 12$ \\
\hline & 600 & $0 \pm 0$ & & 178 & $10 \pm 3$ \\
\hline & & & & 267 & $0 \pm 0$ \\
\hline
\end{tabular}

Tabla 5.4: BER de la modulación Sweep paramétrico para un conjunto de señales medidas. 



\section{Conclusiones}

En esta tesis se estudiaron las diferentes técnicas de modulación abordando la generación paramétrica aplicadas a las comunicaciones submarinas.

En el Capítulo 1, se presentaron de forma rigurosa, todos aquellos principios que rigen la acústica no lineal. Se partió en primera instancia, con la ecuación de la propagación de ondas acústicas lineales (pequeña amplitud) para posteriormente, explicar todos aquellos fenómenos que rigen el comportamiento no lineal, con la aproximación de segundo orden, haciendo énfasis en la ecuación de Westervelt y Burguers, hasta llegar, a la excitación modulada en un medio viscoso y abordar, efecto paramétrico con el estudio de la ecuación de Westervelt, Berktay y la KZK. Todos estos principios sentaron las bases del entendimiento de la propagación acústica no lineal. Además, se validó la relación de amplitud entre el haz secundario y el haz primario medidos experimentalmente con un modelo teórico. El análisis se realizó de dos formas; la primera, estudiando las presiones recibidas del haz primario y secundario y la segunda, a través de las frecuencias emitidas. En el primer análisis se utiliza la ecuación de la ganancia paramétrica compleja, y la segunda, a través de las frecuencias emitidas experimentalmente utilizando la ecuación de la amplitud del haz secundario. Por último, se valida en qué régimen de operación para fuentes paramétricas se encuentra el estudio presentado, cumpliéndose el caso del límite absorción en la zona eférica. También, se introdujeron aquellos fenómenos que afectan la propagación de la señal en el canal submarino y se presenta un ejemplo de de pérdidas por transmisión en aguas poco profundas, según el modelo de Marsh-Shulkin.

En el Capítulo 2, se abordó en primer lugar de forma breve y generalizada, las ventajas de los diferentes tipos de comunicaciones existentes, con el fin de justificar la elección de las comunicaciones acústicas submarinas y aprovechar las características que 
ofrece la generación acústica paramétrica en el agua. Posteriormente, se presentaron diferentes técnicas de modulación que habitualmente, se emplean en el campo de las comunicaciones acústicas, para con ello, enfocarlas desde el punto de vista de la generación paramétrica. Como se vio, la mayoría de las modulaciones lineales están formadas por la combinación de tonos (senos), por lo que, en esta tesis se analizaron las modulaciones paramétricas como un conjunto de dos señales: senos (tonos) y sweeps (señales de banda ancha). Así, se presentó en este capítulo, el proceso de generación de ambos conjuntos de señales partiendo del proceso directo explicado en el Capítulo 1 con el fin de, abordar en el Capítulo 3 las características en lo concerniente a la detección a través del procesado de señales acústicas.

En el Capítulo 3 se estudiaron los procesos en el dominio del tiempo, frecuencia y correlación, que se le aplican a las señales estudiadas en cuestión (senos y sweeps), tanto para la frecuencia primaria (haz primario) como para la paramétrica (haz secundario). El método de la correlación cruzada, ofreció la capacidad de detectar las señales aun enmascaradas por las reflexiones provenientes de la piscina o la balsa. Además de indicar, el tiempo de llegada de la señal (tiempo de vuelo). Al estudiar la detección de senos y sweeps paramétricos, se observó que al ser los sweeps señales de banda ancha (barridos senoidales o tonos) el pico de detección es más estrecho y por lo tanto, su detección es más precisa a diferencia de los tonos paramétricos cuyo pico de detección es ancho, lo que en la mayoría de los casos imposibilita una adecuada detección de la señal, tal como se vio en los resultados del Capítulo 4.

El Capítulo 4, presentó una serie de medidas experimentales realizadas en balsa y en piscina, que permitieron calibrar y caracterizar el campo acústico de un transductor en el régimen lineal y no lineal y comparar los resultados entre ellos, para corroborar las características de la generación paramétrica así como sus ventajas. Se observó que, la señal paramétrica adquirió características de la señal primaria, tal que, la directividad para ambas señales se encuentra entre los $\pm 10^{\circ}$, además de la ausencia de los lóbulos laterales. Respecto a la variación de voltaje, también se observó que para las señales primarias existe un comportamiento lineal, mientras que para las paramétricas un comportamiento exponencial, a partir de los $300 \mathrm{mV}$. Por último, se tiene que con la atenuación, se observó una disminución en la amplitud para las señales primarias y otra menor para las secundarias, aunque con poca diferencia entre ambas; para que este efecto fuera mas notorio, se debe medir a mayores distancias para que el haz primario sea totalmente absorbido por el medio. Con todo ello, se tiene que la generación paramétrica se puede emplear para las comunicaciones acústicas tal como varios autores ya lo han estudiado y comprobado.

Por último, en el Capítulo 5, se presentó otro conjunto de medidas experimentales basadas en la emisión y recepción de diferentes tipos de modulaciones que como se comentó, fueron señales tipo senos y sweeps paramétricos, con los transductores 
enfrentados. Estas señales se realizaron concatenando una cadena de 16 bits (1 y 0 ), cuyo objetivo fue determinar el bit error para cada señal y estudiar su capacidad de detección y compararlo entre las señales moduladas con senos paramétricos (ASK, FSK, PSK) y los sweeps paramétricos. En ello, se observó lo estudiado en el Capítulo 3, donde claramente de entre todas las señales medidas, la que presenta una mejor deteccción sin importar el tiempo de bit enviado fueron los sweeps, debido a que su pico de correlación es bastante estrecho y definido en cada uno de los tiempos esperados.

En general, todos estos estudios demuestran que, aplicando correctamente las técnicas de procesado a la generación acústica paramétrica, se pueden utilizar en sistemas de redes de comunicaciones en aguas poco profundas, donde las múltiples reflexiones que allí se generan, degradan el mensaje enviado, lo que posibilita, con el uso de estas técnicas, una mejora en la recepción del mensaje dado que las señales paramétricas son muy directivas a baja frecuencia, permitiendo que el mensaje se reciba con la mínima interferencia. Además, la rápida absorción de las altas frecuencias en el medio permiten que la baja frecuencias (haz secundario) pueda propagarse a distancias mayores con un ancho de haz bastante estrecho del orden de los $\pm 10^{\circ}$ para las señales estudiadas en esta tesis. Esta es una diferencia muy notable con respecto al uso de transductores convencionales, que generalmente suelen presentar un ancho de haz de aproximadamente $60^{\circ}$ a estas frecuencias. 



\section{Contribuciones científicas}

A continuación se presentan las publicaciones derivadas del trabajo de esta tesis.

\section{JCR Artículo enviado}

María Campo-Valera, Ivan Felis and Isidro Villó-Pérez. Signal processing for parametric acoustic sources applied to underwater communications. Sensors, 2020

\section{JCR Artículo}

María Campo-Valera, Miguel Ardid, Dídac D. Tortosa, Ivan Felis, Juan A. Martínez-Mora, Carlos D. Llorens and Pablo Cervantes. Acoustic parametric signal generation for underwater communication. Sensors, 18(7):2149, 2018

\section{Revista Nacional}

María Mercedes Campo-Valera, Juan Antonio Martínez-Mora and Iván Felis-Enguix. Simulación y diseño de un sensor acústico piezoeléctrico para detección de partículas. Revista de Acústica, pages 1457-1465, 2017. ISSN 2340-7441 


\section{Conferencias}

María Campo-Valera and Ivan Felis. Underwater acoustic communication for the marine environment's monitoring. En Multidisciplinary Digital Publishing Institute Proceedings, ECSA 6th volume 42, page 51, 2019. doi: 10.3390/ecsa-6-06642

María Mercedes Campo-Valera and Ivan Felis-Enguix. Propiedades y aplicaciones del efecto paramétrico en aire y agua. En IV Jornadas JAAS de Acústica Audio y Sonido. Universidad Tres de Febrero (UNTREF), Ingeniería de Sonido, 2018. ISBN 978-987-4151-93-3

María Mercedes Campo-Valera and Ivan Felis-Enguix. Técnicas de modulación para las comunicaciones acústicas no lineales. In Programa de Ingeniería Acústica. En $I V$ Jornadas JAAS de Acústica Audio y Sonido. Universidad Tres de Febrero (UNTREF), Ingeniería de Sonido, 2018. ISBN 978-987-4151-93-3.

María Mercedes Campo-Valera, Miguel Ardid-Ramírez, Dídac D. Tortosa, Ivan Felis-Enguix, and Juan Antonio Martínez-Mora. Estudio del efecto paramétrico en agua con aplicación a un calibrador acústico para detección de neutrinos. En Tecniacústica 2017: $48^{\circ}$ Congreso Español de Acústica; Encuentro Ibérico de Acústica; European Symposium on Underwater Acoustics Applications; European Symposium on Sustainable Building Acoustics: A Coruña 3-6 Octubre 2017, pages 1466-1472. Sociedad Española de Acústica, 2017

María Campo-Valera, Miguel Ardid, Dídac D. Tortosa, Ivan Felis, Carlos D. Llorens and Juan A. Martínez-Mora. Underwater communication using acoustic parametric arrays. En Multidisciplinary Digital Publishing Institute Proceedings ECSA 4th, volume 2, page 139, 2017. doi: 10.3390/ecsa-4-04907 


\section{Apéndice A}

\section{Notas matemáticas}

\section{A.1 Ecuación de estado}

Suponiendo que la presión depende tan sólo de la densidad, esto es, si $p(\rho)$, se puede usar el desarrollo de Taylor,

$$
f(x)=f\left(x_{o}\right)+f^{\prime}\left(x_{o}\right)\left(x-x_{o}\right)+\frac{f^{\prime \prime}\left(x_{o}\right)\left(x-x_{o}\right)^{2}}{2 !}+\frac{f^{\prime \prime \prime}\left(x_{o}\right)\left(x-x_{o}\right)^{3}}{3 !}+\cdots
$$

para desarrollar la ecuación de estado $p=p(\rho)$ en torno al valor de equilibrio $\rho_{o}$,

$$
p=p_{o}+\frac{d p\left(\rho_{o}\right)}{d \rho}\left(\rho-\rho_{o}\right)+\frac{d^{2} p\left(\rho_{o}\right)}{d \rho^{2}} \frac{\left(\rho-\rho_{o}\right)^{2}}{2 !}+\frac{d^{3} p\left(\rho_{o}\right)}{d \rho^{3}} \frac{\left(\rho-\rho_{o}\right)^{3}}{3 !}+\cdots
$$

Introduciendo ahora las variables acústicas $p^{\prime}=p-p_{o}$ y $\rho^{\prime}=\rho-\rho_{o}$, se puede escribir,

$$
p^{\prime}=\frac{d p\left(\rho_{o}\right)}{d \rho} \rho^{\prime}+\frac{1}{2 !} \frac{d^{2} p\left(\rho_{o}\right)}{d \rho^{2}} \rho^{\prime 2}+\frac{1}{3 !} \frac{d^{3} p\left(\rho_{o}\right)}{d \rho^{3}} \rho^{\prime 3}+\cdots
$$

o de forma equivalente,

$$
p^{\prime}=\rho_{o} \frac{d p\left(\rho_{o}\right)}{d \rho}\left(\frac{\rho^{\prime}}{\rho_{o}}\right)+\frac{1}{2 !} \rho_{o}^{2} \frac{d^{2} p\left(\rho_{o}\right)}{d \rho^{2}}\left(\frac{\rho^{\prime}}{\rho_{o}}\right)^{2}+\frac{1}{3 !} \rho_{o}^{3} \frac{d^{3} p\left(\rho_{o}\right)}{d \rho^{3}}\left(\frac{\rho^{\prime}}{\rho_{o}}\right)^{3}+\cdots
$$

Se definen las siguientes cantidades,

$$
A \equiv \rho_{o}\left(\frac{d p}{d \rho}\right)_{\rho_{o}}, \quad B \equiv \rho_{o}^{2}\left(\frac{d^{2} p}{d \rho^{2}}\right)_{\rho_{o}}, \quad C \equiv \rho_{o}^{3}\left(\frac{d^{3} p}{d \rho^{3}}\right)_{\rho_{o}}
$$


pudiendo escribir,

$$
p^{\prime}=A\left(\frac{\rho^{\prime}}{\rho_{o}}\right)+\frac{1}{2 !} B\left(\frac{\rho^{\prime}}{\rho_{o}}\right)^{2}+\frac{1}{3 !} C\left(\frac{\rho^{\prime}}{\rho_{o}}\right)^{3}+\cdots
$$

La cantidad $A$, recibe el nombre de módulo de elasticidad de volumen. Se expresa en $\mathrm{N} / \mathrm{m}^{2}$, las mismas unidades que se usa para la presión. Esta cantidad está relacionada con la velocidad del sonido $c_{o}$ a través de la relación $c_{o}=\sqrt{A / \rho_{o}}$. De donde,

$$
A=\rho_{o} c_{o}^{2}
$$

Para la mayoría de las aplicaciones de interés es suficiente considerar solamente los dos primeros términos del desarrollo (A.5) pudiendo escribir la ecuación de estado, después de ordenar términos como:

$$
p^{\prime}=c_{o}^{2} \rho^{\prime}+\frac{c_{o}^{2}}{\rho_{o}} \frac{B}{2 A} \rho^{2}
$$

\section{A.2 Formación del choque en problemas de frontera}

Para calcular la distancia de choque en problemas de contorno, se parte de la solución de Riemann (1.76) que aquí se repite por comodidad:

$$
p^{\prime}(x, t)=f\left[t-\frac{x}{c\left(p^{\prime}\right)}\right]
$$

Se define la variable $\zeta$ como:

$$
\zeta=t-\frac{x}{c\left(p^{\prime}\right)} \Longrightarrow p(\zeta)=f(\zeta)
$$

Usando la regla de la cadena se obtiene:

$$
\begin{aligned}
\frac{\partial p^{\prime}}{\partial t} & =\frac{d f}{\partial \zeta} \frac{\partial \zeta}{\partial t}=\left(1-x \frac{d c^{-1}}{d p^{\prime}} \frac{\partial p^{\prime}}{\partial t}\right) \frac{d f}{\partial \zeta} \\
& =\left(1+x \frac{1}{c^{2}} \frac{d c}{d p} \frac{\partial p^{\prime}}{\partial t}\right) \frac{d f}{\partial \zeta}
\end{aligned}
$$

Despejando $\partial p^{\prime} / \partial t$ resulta,

$$
\frac{\partial p^{\prime}}{\partial t}=\frac{c^{2}\left(p^{\prime}\right) f^{\prime}(\zeta)}{c^{2}\left(p^{\prime}\right)-c^{\prime}\left(p^{\prime}\right) f(\zeta) x}
$$


Esta derivada diverge dando lugar a una tangente vertical $(t v)$ si el denominador se hace cero. Esto ocurre si $c^{2}\left(p^{\prime}\right)-c^{\prime}\left(p^{\prime}\right) f(\zeta) x=0$. De donde,

$$
x_{t v}=\frac{c^{2}\left(p^{\prime}\right)}{c^{\prime}\left(p^{\prime}\right) f^{\prime}(\zeta)}
$$

Sustituyendo (1.63) en (A.13) resulta,

$$
x_{t v}=\frac{\left(c_{o}+a p^{\prime}\right)^{2}}{a f^{\prime}(\zeta)}
$$

Suponiendo $a p^{\prime}<<c_{o}$, se puede escribir,

$$
x_{t v}=\frac{c_{o}^{2}}{a f^{\prime}(\zeta)}
$$

El valor más pequeño de $x_{t v}$ determina la posición del choque,

$$
x_{s h}=\frac{c_{o}^{2}}{a f_{\max }^{\prime}(\zeta)}=\frac{\rho_{o} c_{o}^{3}}{\beta f_{\max }^{\prime}(\zeta)}
$$

donde se ha usado $a=\beta / \rho_{o} c_{o}$ sengún (1.63).

\section{A.3 Solución Bessel-Fubini caso mono-frecuencia}

Como bien se sabe, si $f(t)$ es una función periódica impar con periodo $T_{o}$, esta puede expresarse mediante una serie de Fourier,

$$
f(t)=\sum_{n=1}^{\infty} b_{n} \sin n \omega_{o} t
$$

donde $\omega_{o}=2 \pi / T_{o}$, y los coeficientes $b_{n}$ están dados por,

$$
b_{n}=\frac{2}{T_{o}} \int_{-T_{o} / 2}^{T_{o} / 2} f(t) \sin \left(n \omega_{o} t\right) d t
$$

Una forma alternativa y más apropiada para este objetivo, es obtener los coeficientes $b_{n}$ a partir de la expresión,

$$
b_{n}=\frac{j 2}{T_{o}} \int_{-T_{o} / 2}^{T_{o} / 2} f(t) e^{-j n \omega_{o} t} d t
$$

Para un $x$ fijo, se propone demostrar que la solución de Riemann (1.81) puede ser desarrollada en serie de Fourier, esto es, encontrar la expresión (1.82). Para ello, se 
parte de dicha solución que aquí se repite por comodidad,

$$
p^{\prime}(x, t)=\mathcal{P}_{o} \sin \left[\omega_{o} t-k_{o} x+\frac{x}{x_{s h}} \frac{p^{\prime}(x, t)}{\mathcal{P}_{o}}\right]
$$

Para un $x$ fijo, se definen las siguientes cantidades,

$$
g(x, t)=\frac{p^{\prime}(x, t)}{\mathcal{P}_{o}}, \quad \sigma=\frac{x}{x_{s h}}, \quad \varphi=\omega_{o} t-k_{o} x
$$

pudiendo escribir (A.20) de una forma más compacta,

$$
g(\varphi)=\sin [\varphi+\sigma g(\varphi)]
$$

el objetivo es expresar $g(\varphi)$ en la forma:

$$
g(\varphi)=\sum_{n=1}^{\infty} b_{n} \sin (n \varphi)
$$

Los coeficientes $b_{n}$ se obtienen por integración:

$$
b_{n}=\frac{j}{\pi} \int_{-\pi}^{\pi} g(\varphi) e^{-j n \varphi} d \varphi
$$

Integrando por partes $\int u d v=u v-\int v d u$, con

$$
\begin{array}{ll}
u=g & \Longrightarrow d u=d g \\
v=-\frac{e^{-j n \varphi}}{j n} \quad \Longrightarrow \quad d v=e^{-j n \varphi} d \varphi
\end{array}
$$

se obtiene,

$$
b_{n}=\frac{1}{n \pi}\left[-\left.g(\varphi) e^{-j n \varphi}\right|_{-\pi} ^{\pi}+\int_{-\pi}^{\pi} e^{-j n \varphi} d g\right]
$$

Por periodicidad, el primer término a la derecha de la igualdad es nulo. Así pues,

$$
b_{n}=\frac{1}{n \pi} \int_{-\pi}^{\pi} e^{-j n \varphi} d g
$$

Ahora, la dificultad reside en encontrar $d g$ para poder integrar en $\varphi$. Para ello se define una nueva variable,

$$
\Phi=\varphi+\sigma g(\varphi)
$$


pudiendo escribir (A.22) como,

$$
g(\Phi)=\sin \Phi
$$

De (A.28) se tiene:

$$
\varphi=\Phi-\sigma \sin \varphi \quad \Longrightarrow \quad d \varphi=(1-\sigma \cos \Phi) d \Phi
$$

Ahora se puede determinar $d g$. Según (A.29),

$$
d g=\cos \Phi d \Phi
$$

Manipulando convenientemente se tiene:

$$
\begin{aligned}
d g=\cos \Phi d \Phi & =\frac{1}{\sigma}[1-(1-\sigma \cos \Phi)] d \Phi \\
& =\frac{1}{\sigma}[d \Phi-(1-\sigma \cos \Phi) d \Phi]
\end{aligned}
$$

Si se observa (A.30) se encuentra finalmente para $d g$,

$$
d g=\frac{1}{\sigma}[d \Phi-d \varphi]
$$

Sustituyendo este resultado en (A.36), se llega a,

$$
b_{n}=\frac{1}{n \pi \sigma}\left[\int_{-\pi}^{\pi} e^{-j n(\Phi-\sigma \sin \Phi)} d \Phi-\int_{-\pi}^{\pi} e^{-j n \varphi} d \varphi\right]
$$

La segunda integral es nula, de modo que,

$$
b_{n}=\frac{1}{n \pi \sigma} \int_{-\pi}^{\pi} e^{j n(\sigma \sin \Phi-\Phi)} d \Phi
$$

Haciendo uso del siguiente resultado para la función de Bessel de primera especie,

$$
J_{n}(x)=\frac{1}{2 \pi} \int_{-\pi}^{\pi} e^{j(x \sin \theta-n \theta)} d \theta
$$

se encuentra,

$$
b_{n}=\frac{2}{n \sigma} J_{n}(n \sigma)
$$


Sustituyendo (A.38) en (A.23) y deshaciendo los cambios dados en (A.21), se tiene finalmente,

$$
p^{\prime}(x, t)=\sum_{n=1}^{\infty} 2 \mathcal{P}_{o} \frac{x_{s h}}{n x} J_{n}\left(\frac{n x}{x_{s h}}\right) \sin n\left(\omega_{o} t-k_{o} x\right)
$$

pudiendo escribir

$$
p^{\prime}(x, t)=\sum_{n=1}^{\infty} B(x) \sin \left[n \omega_{o}\left(t-\frac{x}{c_{o}}\right)\right]
$$

con

$$
B_{n}(x)=2 \mathcal{P}_{o} \frac{x_{s h}}{n x} J_{n}\left(\frac{n x}{x_{s h}}\right)
$$




\section{Apéndice $B$}

\section{Efectos asociados a la viscosidad}

\section{B.1 Ecuación de Westervelt y Burgers viscosa}

Para obtener la ecuación de Westervelt viscosa es necesario reemplazar la ecuación de Euler (1.35) por la ecuación de Navier-Stokes añadiendo una fuerza viscosa $\vec{f}_{\mu}(\vec{r}, t)=\mu \nabla^{2} \vec{u}+\left(\mu_{b}+\frac{1}{3} \mu\right) \vec{\nabla}(\vec{\nabla} \cdot \vec{u})$, donde $\mu$ es la viscosidad dinámica o absoluta y $\mu_{b}$ la viscosidad de volumen o segundo coeficiente de viscosidad. La ecuación de continuidad (1.34) no se modifica y el modelo hidrodinámico toma la forma:

$$
\begin{aligned}
& \frac{\partial \rho}{\partial t}+\vec{\nabla} \cdot(\rho \vec{u})=0 \\
& \rho\left[\frac{\partial \vec{u}}{\partial t}+(\vec{u} \cdot \vec{\nabla}) \vec{u}\right]=-\vec{\nabla} p+\mu \nabla^{2} \vec{u}+\left(\mu_{b}+\frac{1}{3} \mu\right) \vec{\nabla}(\vec{\nabla} \cdot \vec{u})
\end{aligned}
$$

Usando la ecuación de estado (1.42) y siguiendo un esquema idéntico al caso no viscoso, se puede obtener el siguiente sistema de ecuaciones a segundo orden:

$$
\left\{\begin{array}{l}
\frac{\partial \rho^{\prime}}{\partial t}+\rho_{o} \vec{\nabla} \cdot \vec{u}^{\prime}=\frac{1}{\rho_{o} c_{o}^{4}} \frac{\partial p^{\prime 2}}{\partial t}+\frac{1}{c_{o}^{2}} \frac{\partial \mathcal{L}}{\partial t} \\
\rho_{o} \frac{\partial \vec{u}^{\prime}}{\partial t}+\vec{\nabla} p^{\prime}=-\frac{1}{\rho_{o} c_{o}^{2}}\left(\mu_{b}+\frac{4}{3} \mu\right) \vec{\nabla}\left(\frac{\partial p^{\prime}}{\partial t}\right)-\vec{\nabla} \mathcal{L} \\
p^{\prime}=c_{o}^{2} \rho^{\prime}+\frac{1}{\rho_{o} c_{o}^{2}} \frac{B}{2 A} p^{\prime 2}
\end{array}\right.
$$


Este sistema es la versión viscosa del sistema de ecuaciones (1.49a)-(1.49b)-(1.49c). Despejando $p^{\prime}$ se tiene la extensión viscosa de la ecuación de onda (1.51):

$$
\nabla^{2} p^{\prime}-\frac{1}{c_{o}^{2}} \frac{\partial^{2} p^{\prime}}{\partial t^{2}}=-\frac{\beta}{\rho_{o} c_{o}^{4}} \frac{\partial^{2} p^{\prime 2}}{\partial t^{2}}-\frac{\delta}{c_{o}^{4}} \frac{\partial^{3} p^{\prime}}{\partial t^{3}}-\left(\nabla^{2} \mathcal{L}+\frac{1}{c_{o}^{2}} \frac{\partial^{2} \mathcal{L}}{\partial t^{2}}\right)
$$

donde $\delta>0$ es la difusividad del sonido:

$$
\delta=\frac{1}{\rho_{o}}\left(\mu_{b}+\frac{4}{3} \mu\right)
$$

Suponiendo que los efectos no lineales locales son despreciables se hace $\mathcal{L}=0$ en (B.4) para obtener la ecuación de Westervelt viscosa:

$$
\nabla^{2} p^{\prime}-\frac{1}{c_{o}^{2}} \frac{\partial^{2} p^{\prime}}{\partial t^{2}}=-\frac{\beta}{\rho_{o} c_{o}^{4}} \frac{\partial^{2} p^{2}}{\partial t^{2}}-\frac{\delta}{c_{o}^{4}} \frac{\partial^{3} p^{\prime}}{\partial t^{3}}
$$

Haciendo uso del cambio de variables dado en (1.55), se obtiene la ecuación de Burgers viscosa que es la extensión de (1.60):

$$
\frac{\partial p^{\prime}}{\partial x}=\frac{\beta p^{\prime}}{\rho_{o} c_{o}^{3}} \frac{\partial p^{\prime}}{\partial \tau}+\frac{\delta}{2 c_{o}^{3}} \frac{\partial^{2} p^{\prime}}{\partial \tau^{2}}
$$

\section{B.2 Acústica lineal con viscosidad}

Las ecuaciones de primer orden que definen la acústica lineal con viscosidad, se obtienen del sistema de ecuaciones (B.3a), (B.3b) y (B.3c). Despreciando los términos de segundo orden, el resultado es:

$$
\left\{\begin{array}{l}
\frac{\partial \rho^{\prime}}{\partial t}+\rho_{o} \vec{\nabla} \cdot \vec{u}^{\prime}=0 \\
\rho_{o} \frac{\partial \vec{u}^{\prime}}{\partial t}+\vec{\nabla} p^{\prime}=-\frac{\delta}{c_{o}^{2}} \vec{\nabla}\left(\frac{\partial p^{\prime}}{\partial t}\right) \\
p^{\prime}=c_{o}^{2} \rho^{\prime}
\end{array}\right.
$$

Despejando $p^{\prime}(x, t)$ se obtiene la ecuación de onda lineal con viscosidad:

$$
\frac{\partial^{2} p^{\prime}}{\partial t^{2}}-c_{o}^{2} \nabla^{2} p^{\prime}=\delta \frac{\partial}{\partial t}\left(\nabla^{2} p^{\prime}\right)
$$




\section{Bibliografía}

[1] P. J. Westervelt, "Scattering of sound by sound," The Journal of the Acoustical Society of America, vol. 29, no. 2, pp. 199-203, 1957.

[2] D. Blackstock, "Approximate equations governing finite-amplitud sound in thermoviscous fluids," General Dynamics Corporation, Tech. Rep., 1963.

[3] M. F. Hamilton and D. T. Blackstock, Nonlinear acoustics, A. Press and E. 1st (31 de octubre de 1997), Eds. ISBN 0123218608, 1998.

[4] R. W. Lardner, "Acoustic saturation and the conversion efficiency of the parametric array," Journal of Sound and Vibration, vol. 82, no. 4, pp. 473-487, 1982.

[5] B. O. Enflo and C. M. Hedberg, Theory of nonlinear acoustics in fluids, S. Netherlands, Ed. ISBN 1-4020-05072-5, 2002.

[6] V. Y. Jiménez, "Análisis, modelado e igualación no lineal de arrays paramétricos acústicos," Ph.D. dissertation, Universidad Politécnica de Madrid, 2016.

[7] P. J. Westervelt, "Parametric acoustic array," The Journal of the Acoustical Society of America, vol. 35, no. 4, pp. 535-537, 1963.

[8] A. O. Akar, "Characteristics and use of a nonlinear end-fired array for acoustics in air," Master's thesis, Naval postgraduate school, Monterrey, California, 2007.

[9] X. Lurton, An introduction to underwater acoustics: principles and applications. Springer Science \& Business Media, 2002. 
[10] H. O. Berktay and B. V. Smith, "End-fire array of virtual acoustic sources produced by the interaction of cylindrically spreading acoustic waves," Electronics Letters, vol. 1, no. 7, p. 202, 1965.

[11] H. O. Berktay and D. J. Leahy, "Farfield performance of parametric transmitters," The Journal of the Acoustical Society of America, vol. 55, no. 3, pp. 539-546, 1974.

[12] E. A. Zabolotskaya, "Quasi-plane waves, in the nonlinear acoustics of confined beams," Sov. Phys. Acoust., vol. 15, pp. 35-40, 1969.

[13] A. Rozanova-Pierrat, "Mathematical analysis of Khokhlov-Zabolotskaya-Kuznetsov (KZK) equation," HAL Id: hal-00112147, 2006 .

[14] V. P. Kuznetsov, "Equations of nonlinear acoustics," Sov. Phys. Acoust., vol. 16, pp. 467-470, 1971.

[15] M. Hajihasani, Y. Farjami, S. Gharibzadeh, and J. Tavakkoli, "A novel numerical solution to the diffraction term in the KZK nonlinear wave equation," in 2009 38th Annual Symposium of the Ultrasonic Industry Association (UIA). IEEE, 2009, pp. 1-9.

[16] P. Cervenka and P. Alais, "Fourier formalism for describing nonlinear self-demodulation of a primary narrow ultrasonic beam," The Journal of the Acoustical Society of America, vol. 88, no. 1, pp. 473-481, 1990.

[17] R. J. Urick, Principles of underwater sound. Los Altos Hills, California: McGraw-Hill Book, 1975.

[18] M. Saldaña Coscollar, "Acoustic system development for neutrino underwater detectors," Ph.D. dissertation, Universitat Politècnica de València, Campus Gandía, 2017.

[19] L. Kopp, D. Cano, E. Dubois, L. Wang, B. Smith, and R. F. W. Coates, "Potential performance of parametric communications," IEEE Journal of oceanic engineering, vol. 25, no. 3, pp. 282-295, 2000.

[20] M. B. Moffett and R. H. Mellen, "Model for parametric acoustic sources," The Journal of the Acoustical Society of America, vol. 61, no. 2, pp. 325-337, 1977.

[21] M. Campo-Valera, M. Ardid, D. D. Tortosa, I. Felis, J. A. Martínez-Mora, C. D. Llorens, and P. Cervantes, "Acoustic parametric signal generation for underwater communication," Sensors, vol. 18, no. 7, p. 2149, 2018. 
[22] L. M. Brekhovskikh, J. P. Lysanov, and Y. P. Lysanov, Fundamentals of ocean acoustics. Springer Science \& Business Media, 2003.

[23] P. C. Etter, Underwater acoustic modeling and simulation. CRC press, 2018.

[24] O. Haug-Trygve, "Acoustic communication for use in underwater sensor networks," Master's thesis, Institutt for elektronikk og telekommunikasjon, 2009.

[25] C.-T. Chen and F. J. Millero, "Speed of sound in seawater at high pressures," The Journal of the Acoustical Society of America, vol. 62, no. 5, pp. 1129-1135, 1977.

[26] C. C. Leroy, "Development of simple equations for accurate and more realistic calculation of the speed of sound in seawater," The Journal of the Acoustical Society of America, vol. 46, no. 1B, pp. 216-226, 1969.

[27] V. A. Del Grosso, "New equation for the speed of sound in natural waters (with comparisons to other equations)," The Journal of the Acoustical Society of America, vol. 56, no. 4, pp. 1084-1091, 1974.

[28] W. D. Wilson, "Extrapolation of the equation for the speed of sound in sea water," The Journal of the Acoustical Society of America, vol. 34, no. 6, pp. 866-866, 1962.

[29] H. Medwin, "Speed of sound in water: a simple equation for realistic parameters," The Journal of the Acoustical Society of America, vol. 58, no. 6, pp. 1318-1319, 1975.

[30] K. V. Mackenzie, "Nine-term equation for sound speed in the oceans," The Journal of the Acoustical Society of America, vol. 70, no. 3, pp. 807-812, 1981.

[31] A. Quazi and W. Konrad, "Underwater acoustic communications," IEEE Communications Magazine, vol. 20, no. 2, pp. 24-30, 1982.

[32] M. Schulkin and J. A. Mercer, "Colossus revisited: a review and extension of the Marsh-Schulkin shallow water transmission loss model (1962)," Washington University Seatle, Applied physics Lab, Tech. Rep., 1985.

[33] F. H. Fisher and V. P. Simmons, "Sound absorption in sea water," The Journal of the Acoustical Society of America, vol. 62, no. 3, pp. 558-564, 1977.

[34] R. E. Francois and G. R. Garrison, "Sound absorption based on ocean measurements: Part I: pure water and magnesium sulfate contributions," The Journal of the Acoustical Society of America, vol. 72, no. 3, pp. 896-907, 1982. 
[35] — - "Sound absorption based on ocean measurements. Part II: boric acid contribution and equation for total absorption," The Journal of the Acoustical Society of America, vol. 72, no. 6, pp. 1879-1890, 1982.

[36] L. Redondo and A. R. Mateo, "Ruido subacuático: fundamentos, fuentes, cálculo y umbrales de contaminación ambiental," Revista Digital del Cedex, no. 186, pp. 73-73, 2017.

[37] board Ocean studies national research council, Ocean noise and marine mammals. National Academies Press (US), 2003.

[38] G. M. Wenz, "Acoustic ambient noise in the ocean: spectra and sources," The Journal of the Acoustical Society of America, vol. 34, no. 12, pp. 1936-1956, 1962.

[39] H. Kuttruff, Room acoustics. CRC Press, 2016.

[40] N. Farr, A. Bowen, J. Ware, C. Pontbriand, and M. Tivey, "An integrated, underwater optical/acoustic communications system," in OCEANS'10 IEEE SYDNEY. IEEE, 2010, pp. 1-6.

[41] F. J. Cañete, J. López-Fernández, C. García-Corrales, A. Sánchez, E. Robles, F. J. Rodrigo, and J. F. Paris, "Measurement and modeling of narrowband channels for ultrasonic underwater communications," Sensors, vol. 16, no. 2, p. $256,2016$.

[42] L. Butler, "Underwater radio communication," Originally published in Amateur Radio, 1987.

[43] R. K. Moore, "Radio communication in the sea," IEEE spectrum, vol. 4, no. 11, pp. $42-51,1967$.

[44] L. Lanbo, Z. Shengli, and C. Jun-Hong, "Prospects and problems of wireless communication for underwater sensor networks," Wireless Communications and Mobile Computing, vol. 8, no. 8, pp. 977-994, 2008.

[45] J. Aparicio, E. García, A. Jiménez, F. Álvarez, and J. Ureña, "Modelos de propagación de señales acústicas en entornos subacuáticos," Departamento de Ing. Electrónica. Universidad de Extremadura. Madrid., 2010.

[46] J. Claus, "Design and development of an inexpensive acoustic underwater communications and control system," Master's thesis, Florida Institute of Technology, 2014.

[47] A. D'amico and R. Pittenger, "A brief history of active sonar," Space and naval warfare systems center San Diego CA, Tech. Rep., 2009. 
[48] M. Chitre, S. Shahabudeen, and M. Stojanovic, "Underwater acoustic communications and networking: recent advances and future challenges," Marine Technology Society Journal, vol. 42, no. 1, pp. 103-116, 2008.

[49] C. E. Nishimura, IUSS Dual Use: Monitoring whales and earthquakes by using SOSUS. Naval Research Laboratory, 1994.

[50] A. Baggeroer, "Acoustic telemetry - An overview," IEEE Journal of oceanic engineering, vol. 9, no. 4, pp. 229-235, 1984.

[51] T. Tsuchiya, Y. Amitani, H. Ochi, and M. Suzuki, "Digital acoustic video image transmission system for deep-sea research submergence vehicle SHINKAI 6500," The Journal of the Acoustical Society of America, vol. 94, no. 3, pp. 1867-1867, 1993.

[52] R. Galvin and R. F. W. Coates, "Analysis of the performance of an underwater acoustic communications system and comparison with a stochastic model," in Proceedings of OCEANS'94, vol. 3. IEEE, 1994, pp. III-478.

[53] M. Stojanovic, "Recent advances in high-speed underwater acoustic communications," IEEE Journal of Oceanic engineering, vol. 21, no. 2, pp. 125-136, 1996.

[54] E. M. Sozer, J. G. Proakis, R. Stojanovic, J. A. Rice, A. Benson, and M. Hatch, "Direct sequence spread spectrum based modem for under water acoustic communication and channel measurements," in Oceans' 99. MTS/IEEE. Riding the Crest into the 21st Century. Conference and Exhibition. Conference Proceedings (IEEE Cat. No. 99CH37008), vol. 1. IEEE, 1999, pp. 228-233.

[55] D. Kilfoyle and L. Freitag, "Spatial modulation in the underwater acoustic channel," Science applications, Tech. Rep., 2004.

[56] I. F. Akyildiz, D. Pompili, and T. Melodia, "Underwater acoustic sensor networks: research challenges," Ad hoc networks, vol. 3, no. 3, pp. 257-279, 2005 .

[57] J. Heidemann, M. Stojanovic, and M. Zorzi, "Underwater sensor networks: applications, advances and challenges," Philosophical Transactions of the Royal Society A: Mathematical, Physical and Engineering Sciences, vol. 370, no. 1958, pp. 158-175, 2012.

[58] J.-H. Cui, J. Kong, M. Gerla, and S. Zhou, "Challenges: building scalable and distributed underwater wireless sensor networks (UWSNs) for aquatic applications," Channels, vol. 45, no. 4, pp. 22-35, 2005. 
[59] I. F. Akyildiz, D. Pompili, and T. Melodia, "State of the art in protocol research for underwater acoustic sensor networks," in Proceedings of the 1st ACM International workshop on underwater networks, 2006, pp. 7-16.

[60] D. Sarriá Gandul, O. Pallarés Valls, and J. del Río-Fernández, "Acoustic modem for underwater sensor networks," Instrumentation viewpoint, no. 17, pp. 29-30, 2014 .

[61] K. Wiedmann, T. Buch, and T. Weber, "Parametric underwater communications," in Proceedings of Meetings on Acoustics ECUA2012, vol. 17, no. 1. Acoustical Society of America, 2012, p. 070021.

[62] R. F. W. Coates, M. Zheng, and L. Wang, "BASS 300 PARACOM: a model underwater parametric communication system," IEEE Journal of Oceanic Engineering, vol. 21, no. 2, pp. 225-232, 1996.

[63] R. Galvin, R. F. W. Coates, L. S. Wang, and R. Stoner, "Measured channel sounding characteristics and their relationship with the performance of a parametric communication system," in OCEANS 96 MTS/IEEE Conference Proceedings. The Coastal Ocean-Prospects for the 21st Century, vol. 2. IEEE, 1996, pp. 826-831.

[64] M. Zheng, R. F. W. Coates, L. Wang, and R. Stoner, "Underwater acoustic communication utilising parametric transduction with M-ary DPSK modulation," in OCEANS 96 MTS/IEEE Conference Proceedings. The Coastal Ocean-Prospects for the 21st Century. IEEE, 1996, pp. 1-7.

[65] G. Loubet, F. Vial, A. Essebbar, L. Kopp, and D. Cano, "Parametric transmission of wide-band signals," in OCEANS 96 MTS/IEEE Conference Proceedings. The Coastal Ocean-Prospects for the 21st Century, vol. 2. IEEE, 1996, pp. 839-844.

[66] Measured channel characteristics and the corresponding performance of an underwater acoustic communication system using parametric transduction, vol. 147, no. 5. IET, 2000.

[67] S. W. Li, "Pre-processing methods for parametric array to generate wideband difference frequency signals," in OCEANS 2008. IEEE, 2008, pp. 1-8.

[68] J. Lee, C. H. Lee, S. W. Lee, J. Shin, and J. W. Jung, "Analysis of highly directional sonar communication system," Journal of the Institute of Electronics and Information Engineers, vol. 49, no. 12, pp. 3-9, 2012.

[69] K. Wiedmann and T. Weber, "Constellation-constrained capacities for parametric underwater communication," in 2013 MTS/IEEE OCEANS-Bergen. IEEE, 2013, pp. 1-7. 
[70] S. W. Li, "Simulation of the acoustic field of broadband parametric sonar," in 2015 International Conference on Electrical, Automation and Mechanical Engineering. Atlantis Press, 2015.

[71] T. Shengyu, Z. Guangping, Z. Xiao, Y. Jingwei, G. Longxiang, and S. Wei, "Under-ice underwater acoustic communication based on direct sequence spread spectrum system with parametric emission," in 2016 IEEE International Conference on Signal Processing, Communications and Computing (ICSPCC). IEEE, 2016, pp. 1-4.

[72] K. Wiedmann and T. Weber, "Asymptotically tight capacity bounds for parametric underwater communications," in 2016 IEEE 17th International Workshop on Signal Processing Advances in Wireless Communications (SPAWC). IEEE, 2016, pp. 1-5.

[73] Y. Cheng, A. Zhao, J. Hui, T. An, and B. Zhou, "Parametric underwater transmission based on pattern time delay shift coding system," Mathematical Problems in Engineering, vol. 2018, 2018.

[74] S. Tang, G. Zhu, J. Yin, X. Zhang, and X. Han, "A modulation method of parametric array for underwater acoustic communication," Applied Acoustics, vol. 145, pp. 305-313, 2019.

[75] R. S. H. Istepanian and M. Stojanovic, Underwater acoustic digital signal processing and communication systems. Springer, 2002.

[76] A. V. Oppenheim, A. S. Willsky, and S. H. Nawab, Señales y Sistemas. Pearson Educación, 1998.

[77] L. W. Couch, Sistemas de comunicación digitales y analógicos. Pearson Educación, 2008.

[78] W. Tomasi, Sistemas de comunicaciones electrónicas. Pearson educación, 2003.

[79] J. L. Rodríguez and S. C. Suero, Modulación de señales digitales. Universidad Politécnica de Sevilla, 1995.

[80] P. M. Puig, Los sistemas de comunicaciones digitales. Universitat Oberta de Catalunya, 2013.

[81] A. A. Rodríguez, F. P. González, J. C. Sueiro, R. L. Valcarce, C. M. Nartallo, and F. P. Cruz, Comunicaciones digitales. Pearson, 2007.

[82] M. Yoneyama, J.-i. Fujimoto, Y. Kawamo, and S. Sasabe, "The audio spotlight: an application of nonlinear interaction of sound waves to a new type of loudspeaker design." The Journal of the Acoustical Society of America, vol. 73, no. 5, pp. 1532-1536, 1983. 
[83] D. Diego Tortosa, "Caracterización de un transductor paramétrico para emular el patrón acústico de neutrinos de energía ultra alta como calibrador del telescopio de neutrinos KM3Net." Master's thesis, Universitat Polit $\tilde{A}$ “cnica de Valencia, Campus Gandía, 2017.

[84] O. Rioul and M. Vetterli, "Wavelets and signal processing," Signal processing magazine, vol. 8, no. 4, pp. 14-38, 1991.

[85] H.-I. Choi and W. J. Williams, "Improved time-frequency representation of multicomponent signals using exponential kernels," Transactions on Acoustics, Speech, and Signal Processing, vol. 37, no. 6, pp. 862-871, 1989.

[86] E. G. Florez, S. C. I. Foix, and L. J. Nebot, "Análisis en tiempo y frecuencia de señales de vibración tomadas al pie del carril durante el paso de un tren," Scientia et technica, vol. 1, no. 35, 2007.

[87] B. Calleja, L. M. d. Río, and J. L. San Emeterio, "Procesado digital de señales ultrasónicas para la determinación de constantes elásticas dinámicas en materiales rocosos," in Acústica 2008 Conference Proceedings. Ultrasounds ; 4. Sociedade Portuguesa de Acústica, 2008.

[88] J. G. Proakis and D. G. Manolakis, Tratamiento digital de señales. Pearson Prentice-Hall, 2007.

[89] S. Adrián-Martínez, M. Bou-Cabo, I. Felis, C. D. Llorens, J. A. Martínez-Mora, M. Saldaña, and M. Ardid, "Acoustic signal detection through the cross-correlation method in experiments with different signal to noise ratio and reverberation conditions," in International Conference on Ad-Hoc Networks and Wireless. Springer, 2014, pp. 66-79.

[90] B. T. Eovino, "Design and analysis of a pvdf acoustic transducer towards an imager for mobile underwater sensor networks," University of California at Berkeley, Tech. Rep., 2015.

[91] L. E. Kinsler, A. R. Frey, A. B. Coppens, and J. V. Sanders, Fundamentals of acoustics, L. E. Kinsler, Ed. Universidad de California, 1999.

[92] C. H. Sherman and J. L. Butler, Transducers and arrays for underwater sound. Springer, 2007, vol. 4.

[93] M. M. Campo-Valera, M. Ardid-Ramírez, D. D. Tortosa, I. F. Enguix, and J. A. Martínez-Mora, "Estudio del efecto paramétrico en agua con aplicación a un calibrador acústico para detección de neutrinos," in Tecniacústica 2017: $48^{\circ}$ Congreso Español de Acústica; Encuentro Ibérico de Acústica; European Symposium on Underwater Acoustics Applications; European Symposium on 
Sustainable Building Acoustics: A Coruña 3-6 Octubre 2017. Sociedad Española de Acústica, 2017, pp. 1466-1472.

[94] I. O. Wygant, M. Kupnik, J. C. Windsor, W. M. Wright, M. S. Wochner, G. G. Yaralioglu, M. F. Hamilton, and B. T. Khuri-Yakub, "50 kHz Capacitive micromachined ultrasonic transducers for generation of highly directional sound with parametric arrays," IEEE transactions on ultrasonics, ferroelectrics, and frequency control, vol. 56, no. 1, pp. 193-203, 2009.

[95] M. Martins, V. Correia, J. M. Cabral, S. Lanceros-Mendez, and J. G. Rocha, "Optimization of piezoelectric ultrasound emitter transducers for underwater communications," Sensors and Actuators A: Physical, vol. 184, pp. 141-148, 2012.

[96] H. Ahn, Y. Hwang, and W. Moon, "Underwater parametric array source transducer composed of PZT rods and thin polymer plate with high power efficiency for wideband sound generation," The Journal of the Acoustical Society of America, vol. 140, no. 4, pp. 3089-3089, 2016.

[97] A. Zhao, Y. Cheng, T. An, and J. Hui, "Covert underwater acoustic communication system using parametric array," Marine Technology Society Journal, vol. 53, no. 1, pp. 20-26, 2019.

[98] technologie Innomar GmbH, "Smart SES-2000, Parametric Sub-bottom Profiler."

[99] Kongsberg, "Parametric sub-bottom profiler TOPAS PS 18."

[100] benthowave instrument Inc., "BII-7543 2019."

[101] tritech International Ltda, "Seaking SBP-Perfilador paramétrico del subsuelo, 0374-SOM-00008, issue: 03."

[102] M. Campo-Valera and I. Felis, "Underwater acoustic communication for the marine environment monitoring," in MDPI Proceedings, ECSA-6 International Elecctronic Conference on Sensors and Applications, vol. 42, no. 1, 2019, p. 51.

[103] M. Ardid, M. Campo-Valera, D. D. Tortosa, I. Felis, C. D. Llorens, and J. A. Martínez-Mora, "Underwater communication using acoustic parametric arrays," MDPI Proceedings, vol. 2, no. 3, p. 139, 2017. 
